\title{
Fermi-Liquid Instabilities at Magnetic Quantum Phase Transitions
}

\author{
Hilbert v. Löhneysen \\ Physikalisches Institut, Universität Karlsruhe, D-76128 Karlsruhe, Germany and \\ Forschungszentrum Karlsruhe, Institut für Festkörperphysik, D-76021 Karlsruhe, Germany \\ Achim Rosch and Matthias Vojta \\ Institut für Theoretische Physik, Universität zu Köln, D-50923 Köln, Germany \\ Peter Wölfle \\ Institut für Theorie der Kondensierten Materie, Universität Karlsruhe, D-76128 Karlsruhe, Germany
}

(Dated: April 13, 2007)

\begin{abstract}
This review discusses instabilities of the Fermi-liquid state of conduction electrons in metals with particular emphasis on magnetic quantum critical points. Both the existing theoretical concepts and experimental data on selected materials are presented; with the aim of assessing the validity of presently available theory. After briefly recalling the fundamentals of Fermi-liquid theory, the local Fermi-liquid state in quantum impurity models and their lattice versions is described. Next, the scaling concepts applicable to quantum phase transitions are presented. The Hertz-MillisMoriya theory of quantum phase transitions is described in detail. The breakdown of the latter is analyzed in several examples. In the final part experimental data on heavy-fermion materials and transition-metal alloys are reviewed and confronted with existing theory.
\end{abstract}

\section{Contents}

I. Introduction

A. Outline and scope of the review

B. Non-Fermi-liquid behavior vs. breakdown of the Fermi-liquid concept

C. Exponent puzzles

D. Non-Fermi-liquid scenarios

II. Landau Fermi-liquid theory

A. The quasiparticle concept

B. Thermodynamic properties

C. Instabilities within a Fermi-liquid description

D. Finite-temperature corrections to Fermi-liquid theory

E. Transport properties

1. Quasiparticle relaxation rate

2. Kinetic equation and dynamic response

3. Electrical resistivity

F. Kondo effect - concept of a local Fermi liquid

1. The Anderson or Kondo impurity

2. Anderson and Kondo lattice models

3. Multi-channel Kondo effect

4. Impurity quantum phase transitions

G. Non-Fermi-liquid behavior from disorder

III. Fermi-liquid instabilities at quantum phase transitions: Theory

A. Classical vs. quantum phase transitions

B. Scaling properties near quantum phase transitions

C. Itinerant fermion systems

1. Definition of the Hertz model

2. Pressure vs. field tuning

3. Scaling equations

4. Solution for $d+z>4$

5. Solution for $d+z=4$

D. Thermodynamic quantities

E. Self-consistent spin-fluctuation theories

F. Transport properties

G. Approach to the QCP from the Fermi-liquid regime

$\mathrm{H}$. Breakdown of the Hertz model of a magnetic QCP

1. Multiple dynamical exponents: FM QCP
2. Infinitely many marginal operators: $\mathrm{AFM} \mathrm{QCP} \mathrm{in}$ $d=2$

3. Self-energy effects close to QCP 27

4. Pseudogaps close to QCP 27

5. Itinerant AFM with $Q=2 k_{F}$

6. Superconductivity 29

I. Breakdown of the Kondo effect in heavy-fermion metals29

1. "Local" QCP within extended DMFT 30

2. Fractionalized Fermi liquid and deconfined criticality

3. Spin-charge separation at the QCP 32

4. One vs. two transitions

J. Disorder effects close to quantum phase transitions 33

1. Harris criterion and fixed points 33

2. Rare regions and quantum Griffiths singularities $\quad 34$

3. Effects of rare regions on metallic QPT 35

4. Metallic quantum glasses 36

IV. Fermi-liquid instabilities at quantum phase transitions: Experiment 36

A. Quantum critical behavior in heavy-fermion systems 37

1. $\mathrm{CeCu}_{6-x} \mathrm{Au}_{x}$ and $\mathrm{CeCu}_{6-x} \mathrm{Ag}_{x} \quad 38$

2. $\mathrm{Ce}_{1-x} \mathrm{La}_{x} \mathrm{Ru}_{2} \mathrm{Si}_{2}$ and $\mathrm{Ce}\left(\mathrm{Rh}_{1-x} \mathrm{Ru}_{x}\right)_{2} \mathrm{Si}_{2}$

3. $\mathrm{CeCu}_{2} \mathrm{Si}_{2}$ and $\mathrm{CeNi}_{2} \mathrm{Ge}_{2}$

4. $\mathrm{CeTIn}_{5}(T=\mathrm{Co}, \mathrm{Rh}, \mathrm{Ir})$

5. $\mathrm{YbRh}_{2}\left(\mathrm{Si}_{1-x} \mathrm{Ge}_{x}\right)_{2} \quad 47$

B. Quantum critical behavior of itinerant transition-metal magnets

1. $\mathrm{Cr}_{1-x} \mathrm{~V}_{x}$

2. $\mathrm{MnSi} \quad 50$

3. $\mathrm{ZrZn}_{2}$

C. Superconductivity near the magnetic-non-magnetic quantum phase transition

53

V. Conclusions

55

Acknowledgments

56

References

56 


\section{INTRODUCTION}

The Fermi-liquid description of metals is one of the most successful theories in condensed matter physics. It can be applied to describe vastly different systems, ranging from liquid ${ }^{3} \mathrm{He}$ to simple metals like copper or gold to complicated compounds like $\mathrm{CeCu}_{6}$, where the Coulomb interaction in strongly localized $f$-electron shells leads to gigantic interaction effects and a hundred-fold increase of the effective masses. Deviations from Fermi-liquid behavior are a central topic in the experimental and theoretical studies of correlated electronic systems, triggered by the discovery of high-temperature superconductivity, the success in synthesizing effectively low-dimensional materials, and the study of compounds which can be tuned through zero-temperature phase transitions.

\section{A. Outline and scope of the review}

In this review we want to give a combined theoretical and experimental overview of the breakdown of Fermiliquid (FL) behavior in the vicinity of magnetic quantum phase transitions. After a summary of Fermi-liquid theory (Sec. II), including the Kondo effect in local-moment and Kondo-lattice systems, we will describe in Sec. III the established theoretical approach to continuous quantum phase transitions (QPT) in metallic systems. This approach was pioneered by Hertz (1976). Recent theories have addressed its inadequacy in a number of important situations, and we will summarize the current status. In Sec. IV, we will then turn to a variety of experimental systems where quantum criticality and non-Fermi-liquid (NFL) behavior have been observed. By carefully examining available results we shall attempt to state where the standard approach of Hertz applies, and for which systems other theories have to be considered.

Due to space restrictions, a number of interesting topics in the field of metallic quantum criticality will be omitted. We will almost exclusively concentrate on threedimensional (3d) metals, i.e., we will not touch upon high-temperature superconductors and other quasi-twodimensional (2d) and quasi-one-dimensional (1d) materials. We will only focus on QPT involving magnetic order, this removes genuine metal-insulator transitions as well as charge-density wave transitions from our agenda. Most of our discussion will be restricted to the paramagnetic and quantum critical regimes of the transitions; we will say little about the long-range ordered phases which pose additional complications (like e.g. non-trivial Goldstone modes). Further, our primary interest is in clean materials where the effect of quenched disorder is weak. We will therefore leave out metallic spin glasses, and will only briefly mention Kondo disorder and quantum Griffiths scenarios as sources of NFL behavior - we refer the reader to recent reviews (Miranda and Dobrosavljević, 2005; Vojta, 2006b). A comprehensive compilation of experimental NFL data was given by Stewart (2001, 2006).

\section{B. Non-Fermi-liquid behavior vs. breakdown of the Fermi-liquid concept}

Before we focus on some theoretical models and experimental systems where the Fermi-liquid phenomenology appears to fail, it is important to discuss the sometimes confusing terminology in this field. The meaning of phrases like "non-Fermi liquid", "Fermi-liquid instability" or "breakdown of Fermi-liquid theory" varies quite substantially depending on the context, the community, or on theoretical prejudices. This review will not be able to avoid this problem completely, especially as we try to emphasize the open questions in this field.

Conceptually, one should carefully distinguish between two quite different statements. The first is the observation of "non-Fermi liquid behavior", i.e., apparent deviations from the Fermi-liquid phenomenology, e.g., from a constant specific-heat coefficient or a $T^{2}$ dependence of the resistivity at low temperatures. This experimental definition should not be confused with the theoretical statement of a "breakdown of Fermi-liquid theory", which implies that the concept of a FL and its underlying assumptions (see Sec. II) have become invalid.

From this point of view it is not surprising that sometimes "non-Fermi-liquid behavior" can be explained using FL concepts. For example, in a disordered FL the low-temperature resistivity displays a $\sqrt{T}$ cusp (Altshuler and Aronov, 1985) instead of the quadratic $T$ dependence of the weakly disordered case. While this nonanalytic behavior is related to the existence of diffusive modes in the disordered system, which are absent in the usual phenomenology of a Fermi liquid, this does not imply a "complete breakdown" of the FL concept. Another example is the theory of Hertz (1976) for magnetic quantum phase transitions in three dimensions (see Sec. III.C) where the relevant low-energy excitations are the usual fermionic quasiparticles and their collective excitations. The magnetic collective excitations become soft at the quantum critical point (QCP), mediating a singular interaction between the quasiparticles and therefore inducing NFL behavior. Nevertheless, methods and concepts of FL theory can still be applied - this underlying assumption is actually the basis of this theory.

What are the theoretical concepts that can replace the Fermi-liquid paradigm in cases where Fermi-liquid theory breaks down and the low-energy excitations do not carry the quantum numbers of fermionic quasiparticles? In many cases, the answer to this question is not known. One can envision at least two possible scenarios. One is that new weakly interacting quasiparticles with different quantum numbers and interactions can be found. Famous examples are Luttinger liquids with purely bosonic excitations, or the fractional quantum Hall effect with quasiparticles with fractional charge. The other possibility is more difficult to treat theoretically, and much less is known in this case: it is conceivable that no welldefined quasiparticles exist at all and all excitations are incoherent. 


\section{Exponent puzzles}

Non-Fermi-liquid behavior manifests itself in the power-law behavior of physical quantities, with exponents different from those of a Fermi liquid. In this section we discuss under what conditions power-law behavior is expected and we comment on the problem of how exponents can be extracted from experimental data.

Power-law behavior of physical quantities is generally expected in the absence of any close-by scale. For example in an ordinary metal one type of power laws - e.g. a linear temperature dependence of the resistivity $\rho(T)$ - is usually observed in the phonon-dominated regime $\omega_{D} \ll T \ll \epsilon_{F}$, where $\omega_{D}$ stands for the Debye frequency, and $\epsilon_{F}$ is the Fermi energy $\left(k_{B}=\hbar=1\right)$. A different set of Fermi-liquid exponents governs physical quantities in the regime $T \ll \omega_{D}, \epsilon_{F}$, with, e.g., $\Delta \rho=\rho(T)-\rho_{0} \sim T^{2}$ or $\Delta \rho \sim T^{5}$ in regimes dominated by electron-electron or electron-phonon scattering, respectively.

While it is sometimes possible to fit a broad crossover regime (e.g., around $T \approx \omega_{D}$ ) with some effective power law, one should not confuse this non-universal and nongeneric crossover effects with a true power-law scaling behavior. From a theoretical point of view, an exponent is well defined, if at least formally a scaling limit exists (e.g., $T / \omega_{D} \rightarrow \infty$ and $T / \epsilon_{F} \rightarrow 0$ ) where the power law can be observed in a broad temperature range. This purely formal condition translates to the experimental requirement that an algebraic behavior can only be established if it extends over a considerable range of, e.g., temperature in a regime where no other relevant scale is expected to exist. Note that it is not required that the regime of power-law behavior extends down to zero temperature. A Fermi liquid, for example, is essentially never the true ground state of a metallic system, the Fermi-liquid fixedpoint can nevertheless govern the physics over several decades in temperature down to an exponentially small temperature where, e.g., superconductivity sets in.

From an experimental point of view, there are various methods to extract exponents from a measurement. As an illustration for the difficulties which arise and for some of the methods used in this context, we discuss briefly how, e.g., an exponent characterizing the $T$ dependence of the resistivity $\rho(T)$ can be determined. Most commonly, the exponent is obtained from a fit to the data, e.g., the $T$ dependence of the resistivity is fitted to a model form $\rho(T)=\rho_{0}+A T^{\alpha}$ for a certain temperature range $T_{\min }<T<T_{\max }$. A general criterion under which conditions such a procedure is reliable cannot be given - this depends crucially on both prefactor and $T$ dependence of the leading corrections to the algebraic behavior which in many cases are not known. Usually, such fits are believed to be reliable if the exponent depends in a certain regime only weakly on $T_{\max }$ and $T_{\min }$, if the fit extends over more than one decade in temperature and if a plot of $\rho(T)$ as a function of $T^{\alpha}$ "looks" linear.

A slightly less biased method which can also be used to investigate crossover phenomena, is the calculation of an effective $T$-dependent exponent, defined by the $\log$ arithmic derivative of the measured quantity, $\alpha(T) \approx$ $d \ell n\left[\rho(T)-\rho_{0}\right] / d \ell n T$. One should keep in mind that, in particular in crossover regimes, this effective exponent may have little physical significance. However, if the data really can be described by a power law in a large temperature regime, then $\alpha(T)$ will be independent of $T$ and coincides with the "true" exponent. A serious problem is the dependence of this procedure on the residual resistivity $\rho_{0}$. It is usually chosen in such a way as to get the least temperature dependence of $\alpha(T)$ - this is a dangerous bias in the interpretation of the data, especially if some deviations from power-law behavior can be expected, e.g., close to but not directly at a quantum phase transition. This bias can be avoided by defining a temperaturedependent exponent $\alpha(T)=1+d \ell n[d \rho(T) / d T] / d \ell n T$, or equivalently, by fitting power laws to the data in small temperature intervals. Obviously, the latter definition of $\alpha(T)$ does not depend on a constant background $\rho_{0}$ but it is numerically very unstable and requires rather precise data. Hence this procedure is rarely employed.

All of the above methods fail if a small scale $\Delta$ exists where the behavior of the measured quantity crosses over from one to another power law. This is a very common situation close to some quantum critical point, where $\Delta$ can be tuned as a function of some control parameter like pressure $p$, magnetic field $B$ or amount of disorder. In this situation a critical behavior of some quantity, e.g. $X(T)=\rho(T)-\rho_{0}$, is expected, which has the scaling form $X(T, p) \sim T^{\alpha} f(T / \Delta(p))$ with $\Delta \propto\left(p-p_{c}\right)^{\alpha^{\prime}}$ in the limit $T, \Delta \rightarrow 0, T / \Delta=$ const (see Sec. III.B for more details and the discussion of other quantities). In this situation, a scaling analysis is the ideal tool to extract the exponents $\alpha$ and $\alpha^{\prime}$ and the asymptotics in the limit $T / \Delta \gg 1$ and $T / \Delta \ll 1$. If scaling holds, the data for various values of the control parameter $p$ can be collapsed on a single curve by plotting $X / T^{\alpha}$ as a function of $T /\left(p-p_{c}\right)^{\alpha^{\prime}}$. This scaling collapse is used to determine the exponents $\alpha$ and $\alpha^{\prime}$. However, meaningful scaling requires that the data from different curves to be collapsed do overlap significantly - a condition not always fulfilled.

\section{Non-Fermi-liquid scenarios}

In a well-defined Fermi liquid, the usual FL exponents show up below a characteristic scale $T^{*}$. $\left(T^{*}\right.$ is non-universal and depends on many parameters, e.g., the strength of electron-phonon interactions.) What are the requirements to observe non-Fermi-liquid exponents down to the lowest temperatures? A trivial answer to this question is that the scale $T^{*}$ has to disappear. This can happen in at least two ways: (i) $T^{*}$ may be tuned to zero, e.g., by approaching a QCP; (ii) $T^{*}$ may be eliminated by strong disorder: if the distribution of $T^{*}$ in the system is sufficiently broad, no characteristic energy can be defined below which macroscopic Fermi-liquid theory is valid and NFL behavior is expected. 
Possibility (i), namely the suppression of $T^{*}$ in the vicinity of a magnetic bulk quantum critical point, is the main topic of this review. In Sec. II.F we will also mention single-impurity critical points which can induce local NFL behavior. Possibility (ii) is covered by the recent review of Miranda and Dobrosavljević (2005).

We note a further route to NFL behavior here: in principle, a stable NFL fixed point (corresponding to a NFL phase) may exist, where the low-energy excitations do not carry the quantum numbers of fermionic quasiparticles. However, we are not aware of any promising candidate for such a fixed point of a metal in $d=3$ (with the exception of gauge field theories, see Holstein, Norton, and Pincus 1973; Varma, Nussinov, and van Saarloos 2002, or metallic states with liquid-crystal-like order, see Oganesyan, Kivelson, and Fradkin 2001). An extensive discussion about NFL fixed points, especially in $d=2$, can be found in the context of theories of NFL behavior in high-temperature superconductors, see e.g. Anderson (1997); Varma, Nussinov, and van Saarloos (2002). Experimentally, MnSi under pressure shows signatures of a NFL phase in $d=3$, see Sec. IV.B.2, however, a theoretical description is not available to date.

\section{LANDAU FERMI-LIQUID THEORY}

Systems of interacting fermions at low temperature have been of interest early on in the development of condensed matter theory. Landau put forth a phenomenological theory of interacting Fermi systems, the Fermiliquid theory or Landau theory, which is based on the concept of quasiparticles (Landau, 1957a,b, 1959). It proposed to map the properties of Fermi systems at low temperature $T$ onto a dilute gas of strongly interacting fermionic excitations. To some extent, a microscopic justification of this picture was given by Landau and others, although a rigorous general mathematical proof is not available. Recent studies of this problem have used the renormalization group (RG) method (Feldman et al., 1993; Shankar, 1994) which can be used to establish rigorous mathematical bounds on the stability of the FL state (Feldman et al., 1993).

In the following we review the salient assumptions and results of FL theory for later reference.

\section{A. The quasiparticle concept}

Let us start by considering the non-interacting system, where the occupation of single-particle states $|\mathbf{k} \sigma\rangle$ with momentum $\mathbf{k}$ is given by

$$
n_{\mathbf{k} \sigma}^{T=0}=\theta\left(k_{F}-k\right)
$$

where $\theta(x)$ is the step function. The Fermi momentum $k_{F}$ is determined by the density of particles $n=$ $\sum_{\mathbf{k} \sigma} n_{\mathbf{k} \sigma}^{T=0}=\frac{k_{F}^{3}}{3 \pi^{2}}$. Let us now imagine that the interaction between the particles is turned on adiabatically. If the low-energy excitation spectrum of the interacting system is in one-to-one correspondence with the Fermi-gas spectrum, and if the ground state retains the full symmetry of the Hamiltonian, the system is termed a "normal Fermi liquid". Note that the interaction will lead to the appearance of collective modes. However, these bosonic excitations occupy a negligible fraction of phase space in the limit of low temperatures and therefore do not spoil the principal one-to-one correspondence of single-particle states. In an ordered state this one-to-one correspondence is lost.

The low-energy single-particle excitations of the Fermi liquid, with quantum numbers $\mathbf{k}$ and $\sigma$, are called "quasiparticles". In the ground state, their distribution function is again $n_{\mathbf{k} \sigma}$ (1). The energy of a quasiparticle, $\epsilon_{\mathbf{k} \sigma}$, is defined as the amount of energy by which the total energy $E$ of the system increases, if a quasiparticle is added to the unoccupied state $|\mathbf{k} \sigma\rangle$,

$$
\epsilon_{\mathbf{k} \sigma}=\frac{\partial E}{\partial n_{\mathbf{k} \sigma}}
$$

where $\partial n_{\mathbf{k} \sigma}$ is the corresponding change of the distribution function. As a consequence of the interaction, the single-particle energies depend on the state of the system, $\epsilon_{\mathbf{k} \sigma}=\epsilon_{\mathbf{k} \sigma}\left\{n_{\mathbf{k}^{\prime} \sigma^{\prime}}\right\}$. The energy of a single lowenergy quasiparticle added to the ground state may be parametrized as

$$
\epsilon_{\mathbf{k} \sigma}\left\{n_{\mathbf{k}^{\prime} \sigma^{\prime}}^{T=0}\right\}=v_{F}\left(k-k_{F}\right)
$$

for an isotropic system at small energies, with $v_{F}=\frac{k_{F}}{m^{*}}$ being the Fermi velocity. The effective mass $m^{*}$ determines the density of states per spin at the Fermi level

$$
N_{0}=\frac{m^{*} k_{F}}{2 \pi^{2}}
$$

(here and in the following we use units where $\hbar=k_{B}=1$, unless explicitly stated). The effect of interactions with other excited quasiparticles on the energy of a specific quasiparticle may be expressed in terms of an effective two-particle interaction function or "Fermi-liquid" interaction $f_{\mathbf{k} \sigma \mathbf{k}^{\prime} \sigma^{\prime}}$

$$
\delta \epsilon_{\mathbf{k} \sigma}=\sum_{\mathbf{k}^{\prime} \sigma^{\prime}} f_{\mathbf{k} \sigma \mathbf{k}^{\prime} \sigma^{\prime}} \delta n_{\mathbf{k}^{\prime} \sigma^{\prime}}
$$

where $\delta n_{\mathbf{k} \sigma}=n_{\mathbf{k} \sigma}-n_{\mathbf{k} \sigma}^{0}$.

For isotropic systems with short-range interaction the FL interaction function only depends on the angle between $\mathbf{k}$ and $\mathbf{k}^{\prime}$ and on the relative spin orientation of $\sigma$ and $\sigma^{\prime}$, and hence may be parametrized as

$$
f_{\mathbf{k} \sigma \mathbf{k}^{\prime} \sigma^{\prime}}=\frac{1}{2 N_{0}} \sum_{\ell=0}^{\infty} P_{\ell}\left(\hat{\mathbf{k}} \cdot \hat{\mathbf{k}}^{\prime}\right)\left[F_{\ell}^{s}+F_{\ell}^{a} \sigma \sigma^{\prime}\right] .
$$

Here $\hat{\mathbf{k}}=\mathbf{k} /|\mathbf{k}| ; \quad \sigma= \pm 1, \quad P_{\ell}(x)$ are the Legendre polynomials, and $F_{\ell}^{s}$ and $F_{\ell}^{a}$ are the dimensionless 
spin-symmetric and spin-antisymmetric "Landau parameters", which characterize the effect of the interaction on the quasiparticle energy spectrum. For Galileian invariant systems the Landau parameter $F_{1}^{s}$ and the effective mass $m^{*}$ are related through $m^{*} / m=1+F_{1}^{s} / 3$.

In a crystal, the symmetry of the system is reduced to discrete rotations/reflections (the elements of the point group of the lattice), and (if spin-orbit interactions can be neglected) rotations in spin space. As a consequence the band structure $\epsilon_{\mathbf{k}}$ and the FL interaction $f_{\mathbf{k} \sigma \mathbf{k}^{\prime} \sigma^{\prime}}$ may be strongly anisotropic. The parametrization of $\epsilon_{\mathbf{k}}$ and of $f_{\mathbf{k} \sigma \mathbf{k}^{\prime} \sigma^{\prime}}$ then requires additional parameters, which weakens the predictive power of FL theory. In applications of FL theory to metals, it is frequently assumed that an isotropic approximation in $3 \mathrm{~d}$ or quasi-2d systems can give a reasonable account of the FL properties.

\section{B. Thermodynamic properties}

The equilibrium distribution function $n_{\mathbf{k} \sigma}^{0}$ at finite temperature $T$ follows from the assumed one-to-one correspondence:

$$
n_{\mathbf{k} \sigma}^{0}=n_{F}\left(\epsilon_{\mathbf{k} \sigma}\right) \equiv \frac{1}{e^{\epsilon_{\mathbf{k} \sigma} / T}+1} .
$$

This is a complicated implicit equation for $n_{\mathbf{k} \sigma}^{0}$ due to the dependence of $\epsilon_{\mathbf{k} \sigma}$ on $\left\{n_{\mathbf{k}^{\prime} \sigma^{\prime}}^{0}\right\}$.

The derivative of the internal energy with respect to temperature yields the specific heat at constant volume. The leading term at $T \ll T_{F}\left(T_{F}=\epsilon_{F}\right.$ is the Fermi temperature) is linear in $T$, as for the free Fermi gas, and given by the (renormalized) density of states

$$
C_{V}=\frac{2 \pi^{2}}{3} N_{0} T=\gamma T .
$$

The spin susceptibility $\chi$ at $T \ll T_{F}$ and the electronic compressibility follow as

$$
\chi=\frac{2 \mu_{m}^{2} N_{0}}{1+F_{0}^{a}}, \quad \frac{d n}{d \mu}=\frac{2 N_{0}}{1+F_{0}^{s}}
$$

where $\mu_{m}$ is the magnetic moment of electrons. $\chi$ and $\frac{d n}{d \mu}$ are affected both by the mass renormalization and by Fermi liquid parameters describing an effective "screening" of the external fields.

\section{Instabilities within a Fermi-liquid description}

Thermodynamic stability requires that the susceptibilities $\chi$ and $d n / d \mu$ be positive, which leads to the requirements $F_{0}^{a, s}>-1$. A general analysis of the stability of the system with respect to any variation of $n_{\mathbf{k} \sigma}$ results in the stability conditions (Pomeranchuk, 1958)

$$
F_{\ell}^{a, s}>-(2 \ell+1), \ell=0,1, \ldots
$$

In the spin-symmetric isotropic case the compressibility $d n / d \mu$ diverges when $F_{0}^{s} \rightarrow-1$, which is an indication of phase separation into a dense and a dilute phase. More common is the case of ferromagnetism, which appears when $F_{0}^{a} \rightarrow-1$. In the case of an instability at $\ell>0$ the corresponding susceptibility of an anisotropic density excitation in k-space diverges, which is termed Pomeranchuk instability. It may lead to an anisotropic deformation of the Fermi surface (for the spin-antisymmetric sector this has been considered by Akhiezer and Chudnovskii, 1976). While spatially uniform Fermi surface deformations can be captured by Fermi-liquid theory, this is more difficult in the case of instabilities (charge and spin density waves) at finite momentum, as the full momentum dependence of $f_{k k^{\prime}}(q)$ becomes important. The critical behavior of the FL properties on approaching a Pomeranchuk instability is discussed in Sec. III.G.

A different class of instabilities is signaled by a singularity in the quasiparticle scattering amplitude at zero total momentum. It usually leads to the formation of pair-correlated ordered states, i.e., unconventional superconductors, which are not a subject of this review.

\section{Finite-temperature corrections to Fermi-liquid theory}

The leading corrections to Fermi-liquid theory at low temperatures $T \ll T_{F}$ have been the subject of extensive theoretical and experimental study. While for a Fermi gas these corrections are of relative magnitude $\left(T / T_{F}\right)^{2}$, collective effects in an interacting Fermi system generally lead to much larger corrections. Thus one finds that the specific-heat coefficient varies with temperature as

$$
\gamma(T)-\gamma(0)= \begin{cases}-g_{3} T^{2} \ln (T) & \text { for } d=3 \\ -g_{2} T & \text { for } d=2\end{cases}
$$

The coefficients $g_{2}$ and $g_{3}$ have been calculated exactly in lowest order perturbation theory (for a review and references see Chubukov, Maslov, and Millis 2006). Subleading corrections for a Fermi gas with weak repulsion have been derived within an RG approach by Aleiner and Efetov (2006). The leading corrections to the spin susceptibility have been found as

$$
\chi(T)-\chi(0)= \begin{cases}-c_{3} T^{2} & \text { for } d=3 \\ -c_{2} T & \text { for } d=2\end{cases}
$$

In contrast to the specific heat, in $d=3$ a non-analytic contribution is absent (Belitz, Kirkpatrick, and Vojta, 1997; Chubukov, Maslov, and Millis, 2006). There is, however, a non-analytic dependence on the wavevector (see Sec. III.H.1) and on magnetic field. In two dimensions the leading $T$-power of the correction is again reduced from $T^{2}$ to $T$ by singular interaction processes (Chubukov et al., 2005).

Experimentally, the best evidence for the above finitetemperature corrections has been reported in ${ }^{3} \mathrm{He}$, where a $T^{2} \ln (T)$ contribution in $\gamma(T)$ could be identified (see Greywall 1983 and references therein). 


\section{E. Transport properties}

\section{Quasiparticle relaxation rate}

At low temperature, $T \ll T_{F}$, there exists a small number of thermally excited quasiparticles, which interact strongly. The decay rate $\tau^{-1}$ of a quasiparticle on top of the filled Fermi sea is dominated by binary collision processes: the considered quasiparticle in state $|1\rangle=\left|\mathbf{k}_{1} \sigma_{1}\right\rangle$ scatters off a partner in state $|2\rangle$, the two particles ending up in final states $|3\rangle$ and $|4\rangle$. The decay rate is given by the golden rule expression

$$
\frac{1}{\tau_{\mathbf{k}_{1} \sigma_{1}}}=2 \pi \sum_{234}^{\prime}|a(1,2 ; 3,4)|^{2} n_{2}^{0}\left(1-n_{3}^{0}\right)\left(1-n_{4}^{0}\right)
$$

where $a(1,2 ; 3,4)$ is the transition amplitude. The summation over momenta and spins is restricted by conservation of momentum, energy, and spin. A full evaluation of $\tau^{-1}$ yields (Baym and Pethick, 1991)

$$
\begin{aligned}
\frac{1}{\tau_{\mathbf{k}}} & =\left(T^{2}+\frac{\epsilon_{\mathbf{k}}^{2}}{\pi^{2}}\right) \frac{\pi^{3}}{64 \epsilon_{F}}\langle W\rangle \\
\langle W\rangle & =\int_{0}^{1} d \cos \frac{\theta}{2} \int_{0}^{2 \pi} \frac{d \phi}{2 \pi}\left(\left|A_{0}(\theta, \phi)\right|^{2}+3\left|A_{1}(\theta, \phi)\right|^{2}\right) .
\end{aligned}
$$

The quantities $A_{0}$ and $A_{1}$ are the dimensionless scattering amplitudes in the singlet and triplet channel $\left[A_{0,1}=\right.$ $\left.2 N_{0} a(1,2 ; 3,4)\right], \theta$ and $\phi$ parametrize the angle between $\mathbf{k}_{1}, \mathbf{k}_{2}$ and the planes $\left(\mathbf{k}_{1}, \mathbf{k}_{2}\right),\left(\mathbf{k}_{3}, \mathbf{k}_{4}\right)$, respectively. In $2 \mathrm{~d}$ systems the prefactor of $T^{2}$ in $\tau_{\mathbf{k}}^{-1}$ is logarithmically enhanced, $\tau_{\mathbf{k}}^{-1} \sim T^{2} \ln \left(T_{F} / T\right)$ (Chubukov et al., 2005).

The forward scattering limit of the quasiparticle scattering amplitude can be expressed as (Landau, 1959)

$$
A^{\alpha}(\theta, \phi=0)=\sum_{\ell} \frac{F_{\ell}^{\alpha}}{1+F_{\ell}^{\alpha} /(2 \ell+1)} P_{\ell}(\cos \theta)
$$

where $\alpha=s, a$ labels the spin symmetric and antisymmetric particle-hole channels, respectively. As the system approaches a phase transition to a state governed by spatially uniform order, such as a ferromagnet, the corresponding component of $A^{\alpha}(\theta, 0)$ tends to diverge. In the case of the ferromagnet, $F_{0}^{a} \rightarrow-1$, and $A_{0}^{a}=$ $F_{0}^{a} /\left(1+F_{0}^{a}\right) \rightarrow \infty$. The quasiparticle scattering is then dominated by ferromagnetic fluctuations, and the relaxation rate $\tau^{-1}$ is expected to scale as $N_{0}^{2} /\left(1+F_{0}^{a}\right)^{2} \propto \chi^{2}$, with $\chi$ the static spin susceptibility.

\section{Kinetic equation and dynamic response}

In the presence of slowly varying disturbances, the system may be described by a quasiclassical distribution function $n_{\mathbf{k} \sigma}(\mathbf{r}, t)$. This is possible as long as the energy and momentum of the quanta of the external field, $\omega$ and $q$, are much smaller than the typical energy and momentum of the quasiparticles, i.e., $\omega \ll T, q \ll T / v_{F}$. The distribution function satisfies the kinetic equation

$$
\partial_{t} n_{\mathbf{k} \sigma}+\nabla_{k} \epsilon_{\mathbf{k} \sigma} \cdot \nabla_{r} n_{\mathbf{k} \sigma}-\nabla_{r} \epsilon_{\mathbf{k} \sigma} \cdot \nabla_{k} n_{\mathbf{k} \sigma}=I\left\{n_{\mathbf{k} \sigma}\right\}
$$

The left-hand side describes the dissipationless flow of quasiparticles in phase space. It goes beyond the Boltzmann equation in that the quasiparticle energy $\epsilon_{\mathbf{k}}(\mathbf{r}, t)$ itself depends on position and time, due to its dependence on the distribution function as given by (5). Among other things, this gives rise to the appearance of collective modes, as well as interesting nonlinear effects (which we will not discuss).

On the r.h.s. of (16) we have the so-called collision integral $I$, which describes the abrupt change of momentum and spin of quasiparticles in a collision process. It is given by $I_{\mathbf{k} \sigma}=-n_{\mathbf{k} \sigma} / \tau_{\mathbf{k} \sigma}^{\text {noneq }}\left(\left\{n_{\mathbf{k} \sigma}\right\}\right)+\left(1-n_{\mathbf{k} \sigma}\right) / \tau^{\text {noneq }}(\{1-$ $\left.n_{\mathbf{k} \sigma}\right\}$ ), which is the number of quasiholes minus the number of quasiparticles in state $|\mathbf{k} \sigma\rangle$ decaying per unit time. The non-equilibrium relaxation rate $1 / \tau_{\mathbf{k} \sigma}^{\text {noneq }}\left(\left\{n_{\mathbf{k} \sigma}\right\}\right)$ is obtained from (13) by replacing $n_{\mathbf{k}_{i} \sigma_{i}}$ and $\epsilon_{\mathbf{k}_{i} \sigma_{i}}$, by their non-equilibrium counterparts.

If the applied external field is weak and one is allowed to linearize in the deviation of the distribution function from its equilibrium value. The resulting linearized and Fourier-transformed kinetic equation is given by

$$
\left(\omega-\mathbf{v}_{\mathbf{k}} \cdot \mathbf{q}\right) \delta n_{\mathbf{k}}(q, \omega)+\mathbf{v}_{\mathbf{k}} \cdot \mathbf{q} \frac{\partial n_{\mathbf{k}}^{0}}{\partial \epsilon_{\mathbf{k}}} \delta \epsilon_{\mathbf{k}}=i \delta I
$$

where $\delta \epsilon_{\mathbf{k} \sigma}(\mathbf{q}, \omega)$ is defined as

$$
\delta \epsilon_{\mathbf{k} \sigma}(\mathbf{q}, \omega)=\sum_{\mathbf{k}^{\prime} \sigma^{\prime}} f_{\mathbf{k} \sigma \mathbf{k}^{\prime} \sigma^{\prime}} \delta n_{\mathbf{k}^{\prime} \sigma^{\prime}}(\mathbf{q}, \omega) .
$$

In principle, $f_{\mathbf{k} \sigma \mathbf{k}^{\prime} \sigma^{\prime}}$ is also a function of $\mathbf{q}$ and $\omega$, and the limit $\mathbf{q}, \omega \rightarrow 0$ is understood here. For a charged system with long-range Coulomb interaction, a classical or Hartree-type interaction part must be separated out, $f_{\mathbf{k} \sigma \mathbf{k} \sigma^{\prime}}(\mathbf{q})=\frac{4 \pi e^{2}}{q^{2}}+\tilde{f}_{\mathbf{k} \sigma \mathbf{k}^{\prime} \sigma^{\prime}}$

Assuming the Landau parameter $F_{0}^{s}$ to be dominant and collision effects to be small, the kinetic equation may be solved in the presence of an external potential $-\delta \mu^{e x t}$ to obtain the density response function:

$$
\frac{\delta n}{\delta \mu^{e x t}}=\chi_{c}(\mathbf{q}, \omega)=\frac{\chi_{0}(\mathbf{q}, \omega)}{1+F_{0}^{s} \chi_{0}(\mathbf{q}, \omega)}
$$

where

$$
\chi_{0}(\mathbf{q}, \omega)=\sum_{\mathbf{k} \sigma} \frac{\mathbf{k} \cdot \mathbf{q}}{\omega-\mathbf{v}_{\mathbf{k}} \cdot \mathbf{q}+i 0} \frac{\partial n_{\mathbf{k}}^{0}}{\partial \epsilon_{\mathbf{k}}} .
$$

This is the well-known random-phase approximation (RPA) form of the density response function in the limit $q \ll k_{F}$.

In analogy to the density response, the dynamical spin susceptibility $\chi_{m}(\mathbf{q}, \omega)$ is defined as the response of the magnetization to a magnetic field, $\chi_{m}=\delta M / \delta\left(\mu_{m} B\right)$, and is given by Eq. (19) with $F_{0}^{s}$ replaced by $F_{0}^{a}$. The 
dynamical structure factor $S(\mathbf{q}, \omega)$, experimentally accessible via magnetic neutron scattering, is related to the dynamical susceptibility $\chi_{m}(\mathbf{q}, \omega)$ through

$$
S(\mathbf{q}, \omega)=[1+n(\omega)] \operatorname{Im} \chi_{m}(\mathbf{q}, \omega+i 0)
$$

where $n(\omega)=\left(e^{\omega / T}-1\right)^{-1}$ is the Bose function.

\section{Electrical resistivity}

As an example of a transport coefficient we consider the electrical conductivity $\sigma=\rho^{-1}$ (where $\rho$ is the resistivity), defined as the response of the electric current density $\mathbf{j}$ to the (screened) electric field $\mathbf{E}, \mathbf{j}=\overleftrightarrow{\sigma} \mathbf{E}$ (assuming cubic symmetry). In terms of the linearized distribution function $\delta n_{\mathbf{k}}=\lim _{\omega \rightarrow 0} \lim _{\mathbf{q} \rightarrow 0} \delta n_{\mathbf{k}}(\mathbf{q}, \omega)$ the conductivity is given by $\sigma_{i j}=e \sum_{\mathbf{k} \sigma} v_{\mathbf{k} i} \delta n_{\mathbf{k}} / E_{j}$. Here $\delta n_{\mathbf{k}}$ satisfies the Boltzmann equation

$$
e \mathbf{v}_{\mathbf{k}} \cdot \mathbf{E}\left(\frac{\partial n_{\mathbf{k}}^{0}}{\partial \epsilon_{\mathbf{k}}}\right)=\delta I\left\{\delta n_{\mathbf{k}}^{\ell}\right\}
$$

where $\delta n_{\mathbf{k}}^{\ell}$ is the deviation of $n_{k}$ from local equilibrium. The collision integral $\delta I$ describes the effect of interquasiparticle collisions and any other collision processes. For a translation invariant system, the quasiparticle collisions are momentum conserving and the resistivity would be zero. The resistivity is finite in a real solid, if Umklapp scattering is possible (for a recent discussion of the role of Umklapp scattering see Rosch 2005). The most important source of momentum dissipation at low $T$ is, however, impurity scattering.

In lowest approximation the collision integral may be modelled as

$$
\delta I=-\hat{C}_{e-e} \delta n_{\mathbf{k}}^{\ell}-\hat{C}_{i} \delta n_{\mathbf{k}}^{\ell}
$$

where $\hat{C}_{e-e}$ and $\hat{C}_{i}$ are the linear integral operators describing electron-electron and electron-impurity collisions, respectively. Taking into account that $\hat{C}_{e-e} \sim$ $\tau^{-1} \sim T^{2}$ at low $T$, one finds for the resistivity

$$
\rho(T)=\rho_{0}+A T^{2}+\ldots
$$

Here $\rho_{0}$ is the residual resistivity from impurity scattering. The coefficient $A$ is given by a weighted angular average of the squared quasiparticle scattering amplitudes $A_{0,1}(\theta, \phi)=2 N_{0} a(1,2 ; 3,4)$, which sensitively depends on the anisotropy of the scattering and the band structure. The resulting transport scattering rate $\tau_{\mathrm{tr}}^{-1}$ is in general different from the single-particle relaxation rate $\tau^{-1}$ (14) (except for isotropic scattering), as only finiteangle scattering affects transport.

Provided that the transition amplitudes $a(1,2 ; 3,4)$ are weakly momentum dependent, i.e., when they are governed by local physics, the ratio of the resistivity coefficient and the square of the specific-heat coefficient, $A / \gamma^{2}$, may be expected to be material-independent since
$A \propto N_{0}^{2}$ and $\gamma \propto N_{0}$. This is indeed observed for a large number of heavy fermion systems (Kadowaki and Woods, 1986), and $A / \gamma^{2}$ is termed the Kadowaki-Woods ratio. A corresponding dimensionless quantity may be defined as

$$
R_{\mathrm{KW}}=\frac{\rho(T)-\rho_{0}}{\rho_{0}} \frac{n^{2}}{(\gamma T)^{2}} .
$$

\section{F. Kondo effect - concept of a local Fermi liquid}

The Kondo problem (Hewson, 1993) goes back to the discovery of a resistance minimum at low temperatures in metals with dilute magnetic impurities. The minimum and low- $T$ increase of the resistance were successfully explained by Kondo (1964) within a perturbative calculation. Within the so-called Kondo (or $s$ d) model, a magnetic impurity is described by a local spin $\mathbf{S}$ (assumed to be $S=\frac{1}{2}$ located at $\mathbf{r}=0$ ) exchange-coupled to the local conduction-electron spin density $\mathbf{s}_{0}=\frac{1}{2} \sum_{\mathbf{k}, \mathbf{k}^{\prime}} \sum_{\sigma \sigma^{\prime}} c_{\mathbf{k} \sigma}^{\dagger} \tau_{\sigma \sigma^{\prime}} c_{\mathbf{k}^{\prime} \sigma^{\prime}}$,

$$
H_{s d}=\sum_{\mathbf{k}, \sigma} \epsilon_{\mathbf{k}} c_{\mathbf{k} \sigma}^{\dagger} c_{\mathbf{k} \sigma}+J \mathbf{S} \cdot \mathbf{s}_{0}
$$

where $\tau_{\sigma \sigma^{\prime}}$ is the vector of Pauli matrices, and $J$ is exchange coupling. Kondo found that the electrical resistivity $\rho$ due to scattering of conduction electrons off the impurity acquired a logarithmic dependence on temperature in third order in $J$

$$
\rho=\rho_{B}\left[1+2 N_{0} J \ln \frac{D}{T}+\ldots\right]
$$

where $\rho_{B} \propto J^{2}$ is the usual Born approximation result, $N_{0}$ is the local conduction electron density of states per spin, and $D$ is the half-width of the conduction band. The reason for the $T$ dependence in $\rho$ lies in the resonant scattering from the degenerate ground state of the magnetic impurity. As seen from the result (27), perturbation theory breaks down below the Kondo temperature

$$
T_{\mathrm{K}}=D \sqrt{N_{0} J} \exp \left(-\frac{1}{N_{0} J}\right),
$$

when the first-order correction term becomes comparable to the Born approximation. The prefactor in (28) is chosen such that a $1 / \ell n^{2}\left(T / T_{\mathrm{K}}\right)$ correction to the magnetic susceptibility $\chi_{\mathrm{imp}}(T)$ is absent for $T \gg T_{K}$. For $J \rightarrow 0$ the Kondo temperature $T_{\mathrm{K}}$ depends on the coupling $J$ in a non-analytic way.

As pointed out by Anderson and collaborators, the ground state of the Kondo model (26) is non-degenerate. The $S=\frac{1}{2}$ impurity spin is fully compensated by a "screening cloud" of conduction electron spins containing in total one electron spin, bound to the impurity in a singlet state. The impurity complex formed in this way acts like a potential scatterer. The low-energy physics of this system has been formulated by Nozières (1974) in terms of a local Fermi liquid picture. 
It is useful to introduce a model of a magnetic impurity in which the electronic structure of the impurity is displayed directly. This Anderson impurity model (Anderson, 1961) consists of an impurity orbital (a $d$ or $f$ orbital of an incompletely filled inner atomic shell) hybridizing with a conduction band,

$$
\begin{aligned}
H= & \sum_{\mathbf{k}, \sigma} \epsilon_{\mathbf{k}} c_{\mathbf{k} \sigma}^{\dagger} c_{\mathbf{k} \sigma}+\epsilon_{f} \sum_{\sigma} f_{\sigma}^{\dagger} f_{\sigma}+U n_{f \uparrow} n_{f \downarrow} \\
& +V \sum_{\mathbf{k}, \sigma}\left(c_{\mathbf{k} \sigma}^{\dagger} f_{\sigma}+\text { h.c. }\right)
\end{aligned}
$$

where $f_{\sigma}^{\dagger}$ creates an electron with spin projection $\sigma$ in the $f$ orbital, $n_{f \sigma}=f_{\sigma}^{\dagger} f_{\sigma}$, and $V$ is the hybridization matrix element. The decisive feature of the model is the strong Coulomb interaction $U$ which leads to localmoment formation: if the impurity level lies below the Fermi energy, $\epsilon_{f}<0$, while $\epsilon_{f}+U>0$ and the bare hybridization width $\Gamma=\pi N_{0} V^{2}$ is small, $\Gamma \ll\left|\epsilon_{f}\right|, \epsilon_{f}+U$, the impurity level is mainly occupied by a single electron (rather than being empty or doubly occupied) and thus represents a local moment of spin $\frac{1}{2}$.

By projecting the Anderson Hamiltonian (29) onto the subspace of singly occupied impurity states (Schrieffer and Wolff, 1966) one is led to the Kondo model with

$$
J=2 V^{2}\left(\frac{1}{\left|\epsilon_{f}\right|}+\frac{1}{\epsilon_{f}+U}\right)>0 .
$$

We note that Kondo physics is not restricted to the screening of magnetic degrees of freedom, it can occur in any situation where transitions between a multi-level impurity are induced through the interaction with a bath of fermionic particles. The particular example of the quadrupolar Kondo effect will be discussed in Sec. II.F.3.

\section{The Anderson or Kondo impurity}

The low- $T$ FL regime of the exactly screened Anderson model can be expected to be continuously connected to the non-interacting limit of the Anderson model, and consequently perturbation theory in powers of $U$ is appropriate (Hewson, 1993). The dynamical properties of the impurity are described by the self-energy $\Sigma_{f \sigma}(\omega)$ of the local $f$ electron, which determines the energy shift and broadening of the poles of the local Green's function

$$
G_{f}(\omega+i 0)=\frac{1}{\omega-\epsilon_{f}+i \Gamma-\Sigma_{f \sigma}(\omega+i 0)}
$$

where a particle-hole symmetric conduction band has been assumed. In the absence of Coulomb interaction, $U \rightarrow 0, \Sigma_{f \sigma}(\omega)=0$. The imaginary part of $\Sigma_{f \sigma}$ measures the decay rate of the $f$ electron into particle-hole pairs: Standard phase-space restrictions dictate the small- $\omega$ behavior,

$$
-\operatorname{Im} \Sigma_{f \sigma}(\omega+i 0) \propto \omega^{2} .
$$

It follows from the Kramers-Kronig relation that

$$
\operatorname{Re} \Sigma_{f \sigma}(\omega)=\Sigma_{f \sigma}(0)+\left(1-Z^{-1}\right) \omega+\mathcal{O}\left(\omega^{2}\right) .
$$

Thus, the Green's function $G_{f \sigma}$ in the limit of small $\omega$ takes the form

$$
G_{f \sigma}(\omega+i 0)=\frac{Z}{\omega-\epsilon_{f \sigma}^{*}+i \Gamma^{*}}
$$

where $\epsilon_{f \sigma}^{*}=Z\left(\epsilon_{f}+\Sigma_{f \sigma}(0)\right)$ and $\Gamma^{*}=Z \Gamma$. Here $Z$ plays the role of the quasiparticle weight factor in FL theory.

The above analysis was restricted to the low- $T$ regime, assuming a FL ground state. To capture the crossover from high to low temperature, the concept of scaling and of the renormalization group (RG) has turned out to be the principal tool. In Anderson's "poor man's scaling" approach (1970), applied to the Kondo model, the Hamiltonian is projected onto a smaller Hilbert space where the conduction electron bandwidth $D_{0}$ has been reduced by an infinitesimal amount $d D$. The effective Hamiltonian still takes the form of the Kondo model, with a modified coupling constant $J(D)$. (Technically, "poor man's scaling" is a momentum-shell RG without the step of rescaling the cutoff and fields.) The flow of the dimensionless coupling $j=N_{0} J$ is described by the so-called $\beta$ function, which can be calculated in a power series in $j$ :

$$
\beta(j)=-\frac{d j}{d \ln D}=j^{2}-\frac{1}{2} j^{3}+\ldots
$$

The radius of convergence of this series is not known a priori. However, from (35) we can infer that for small antiferromagnetic $j>0$ the effective coupling grows under the reduction of the bandwidth. In contrast, a ferromagnetic coupling $(j<0), j$ scales to zero for $D \rightarrow 0$, leaving a free spin and a free conduction band. This solves the problem for ferromagnetic coupling; the finitetemperature thermodynamics displays logarithmic corrections to the free-spin behavior due to the flow of $j$ to zero. For the antiferromagnetic case, which is the usual situation, there are obviously two possibilities: (i) the coupling tends to a finite value as $D \rightarrow 0$, (ii) the coupling grows indefinitely, $j \rightarrow \infty$. It was recognized early on by Anderson and coworkers (Anderson, 1970; Anderson and Yuval, 1970) that the latter scenario is realized in the usual (single-channel) Kondo situation.

Using the fact that the fixed point of $j \rightarrow \infty$ is indeed stable (Nozières, 1974), one may now discuss the local properties of conduction electrons at the impurity, taking into account (i) the energy shift provided by the scattering off the static potential of the fully screened impurity, (ii) the interaction between conduction electrons at the impurity site induced by virtual excitations of the impurity complex. This is elegantly done in terms of a phenomenological description following the Landau FL theory (Nozières, 1974), which provides a correct and useful picture of the Kondo behavior at small temperatures and fields. In order to describe the crossover 
from high-temperature local-moment physics to the lowtemperature Kondo-screened state, more elaborate methods are required. The thermodynamic properties of the Kondo model (26) and the Anderson model (29) may be calculated exactly by using the Bethe ansatz method (Andrei, 1980; Andrei, Furaya, and Lowenstein, 1983; Wiegmann, 1980). The dependence of the free energy and its derivatives on temperature and magnetic field is found to exhibit single parameter scaling behavior in $\left(T / T_{\mathrm{K}}\right)$ and $\left(B / T_{\mathrm{K}}\right)$ from the low-energy regime below $T_{\mathrm{K}}$ up to high energies $\gg T_{\mathrm{K}}$, but sufficiently below the conduction electron bandwidth. Most importantly, there is no phase transition upon variation of $T$ or $B$ for the metallic single-impurity Kondo problem (see Sec. II.F.4 for phase transitions in impurity models).

Dynamical properties can be calculated numerically using Wilson's renormalization group method (NRG) (Krishna-murthy, Wilkins, and Wilson, 1980; Wilson, 1975). The local $f$-electron spectral function $A_{f}(\omega)$ has been determined by NRG as well, both at $T=0$ (Costi and Hewson, 1990; Sakai, Shimizu, and Kasuya, 1989) and at finite $T$ (Costi, Kroha, and Wölfle, 1996), and by self-consistent diagrammatic methods (Kroha and Wölfle, 2005). The electrical resistivity for the Anderson model was found to obey single-parameter scaling behavior in $T / T_{\mathrm{K}}$ (Costi and Hewson, 1992).

\section{Anderson and Kondo lattice models}

So far we have considered the properties of a single quantum impurity in a host metal. We now turn to a discussion of materials where "quantum impurity ions" are put on a lattice. Generalizing the single-channel Anderson impurity model (29) to a lattice of localized orbitals, $f_{i}$, one obtains the so-called periodic Anderson model (PAM),

$$
\begin{aligned}
H & =\sum_{\mathbf{k} \sigma} \epsilon_{\mathbf{k}} c_{\mathbf{k} \sigma}^{\dagger} c_{\mathbf{k} \sigma}+\epsilon_{f} \sum_{i \sigma} f_{i \sigma}^{\dagger} f_{i \sigma}+ \\
& +U \sum_{i} n_{i \uparrow}^{f} n_{i \downarrow}^{f}+V \sum_{i \sigma}\left(f_{i \sigma}^{\dagger} c_{i \sigma}+\text { h.c. }\right) .
\end{aligned}
$$

Both direct hopping of and direct exchange between $f$ electrons are neglected here. In a situation of large $U$ and negative $\epsilon_{f}$ local moments on the $f$ sites become well defined. Employing a Schrieffer-Wolff transformation as in the single-impurity case, the PAM maps onto the Kondo lattice model

$$
H=\sum_{\mathbf{k} \sigma} \epsilon_{\mathbf{k}} c_{\mathbf{k} \sigma}^{\dagger} c_{\mathbf{k} \sigma}+J \sum_{i} \mathbf{S}_{i} \cdot \mathbf{s}_{i}
$$

where the Kondo coupling $J$ is related to the parameters of the Anderson model through (30).

We start by discussing qualitative features of the Anderson and Kondo lattice models. For small interaction $U$, the periodic Anderson model (36) can be expected to describe a Fermi liquid with two bands. As detailed

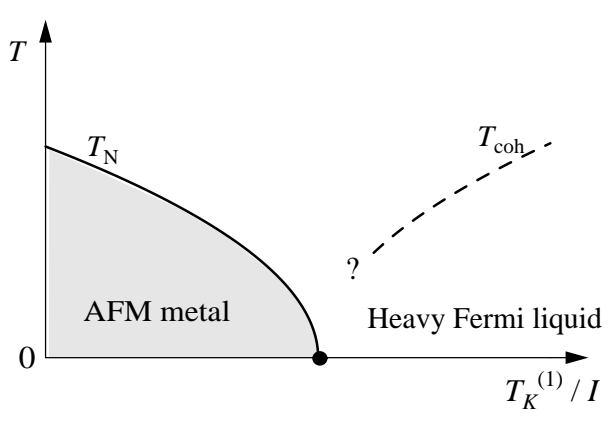

FIG. 1 Doniach's phase diagram of the Kondo lattice, as function of the ratio of Kondo $\left(T_{K}^{(1)}\right)$ and inter-moment exchange $(I)$ energies - here $T_{K}^{(1)}$ is the single-impurity Kondo scale, measuring the strength of the Kondo effect. The heavy Fermi liquid is formed below the coherence temperature $T_{\text {coh }}$. The behavior of $T_{\text {coh }}$ across the phase transition will be discussed in Sec. III.I.

below, a Fermi liquid at lowest temperatures can also survive in the large- $U$ limit and thus in the Kondo-lattice case - this requires the local moments to be screened by a lattice generalization of the Kondo effect. The resulting Fermi liquid, formed below a coherence temperature $T_{\text {coh }}$, will have a Fermi volume containing both $c$ electrons and local moments (dubbed "large Fermi volume"), consistent with the Luttinger theorem (Oshikawa, 2000). The resistivity will follow the usual quadratic $T$ dependence (24). It is interesting to discuss the evolution of this state with temperature: For $T \gg T_{\text {coh }}$ (but still assuming well-formed local moments) the system can be described as $c$ fermions with a "small" Fermi volume interacting weakly with a paramagnetic system of localized spins. The resistivity is often rather low in this situation. In the crossover region $T \sim T_{\text {coh }}$ the Fermi surface fluctuates strongly, giving rise to a very high resistivity of the order of the unitarity limit - experimentally, this resistivity maximum is often used to define $T_{\text {coh }}$.

The screening of the local moments, required for FL behavior in the Kondo lattice, competes with interactions between the local moments. Such interactions can be due to direct hopping or exchange between the $f$ orbitals, but are also generated due to the polarization of the conduction electrons. This indirect Ruderman-Kittel-KasuyaYosida (RKKY) interaction is given in lowest (quadratic) order in $J$ by

$$
\begin{aligned}
H_{\mathrm{RKKY}} & =\sum_{i, j} I_{i j} \quad \mathbf{S}_{i} \cdot \mathbf{S}_{j}, \\
I_{i j} & =N_{0} J^{2} F\left(k_{F} R_{i j}\right)
\end{aligned}
$$

where $F(x)=(x \cos x-\sin x) / x^{4}$ and $R_{i j}$ is the distance between lattice sites $i, j$. In the Kondo-screened state $I_{i j}$ is expected to be renormalized, in particular at long distances, but a reliable determination of $I_{i j}$ is not available at present. The competition between the Kondo coupling and the inter-moment interaction will 
govern the phase diagram of the Kondo lattice (Doniach, 1977). The most natural competitor of the Fermi liquid is a magnetically ordered metal, Fig. 1, but in the presence of strong quantum effects and geometric frustration spin-glass and spin-liquid states may also occur.

We continue with an analysis of the FL phase of the periodic Anderson model - here non-local interaction effects are believed to be unimportant. A viable method is perturbation theory in $U$ (Hewson, 1993). Since the interaction $U$ is assumed to act only between electrons in the $f$ level, there is only one self-energy $\Sigma_{\sigma}(\mathbf{k}, \omega)$. Expanding $\Sigma$ near the Fermi energy $\omega=0$ and near the Fermi surface $k=k_{F}$ (assuming an isotropic band structure) and using the fact that $\operatorname{Im} \Sigma \sim \omega^{2}$ in this region, one may define a quasiparticle component of the singleparticle Green's function $G^{f f}(\mathbf{k}, \omega)$ of weight

$$
Z=\left[1-\frac{\partial \Sigma\left(k_{F}, \omega\right)}{\partial \omega}\right]_{\omega=0}^{-1}
$$

located at energy

$$
\tilde{\epsilon}_{f, \mathbf{k}}=Z\left[\epsilon_{f}+\Sigma\left(\mathbf{k}_{F}, 0\right)+\left(\mathbf{k}-\mathbf{k}_{F}\right) \cdot \nabla_{k} \Sigma\right]
$$

and hybridizing with the conduction band with renormalized strength $\tilde{V}=Z^{1 / 2} V$. One finds two quasiparticle bands with dispersion

$$
\tilde{\epsilon}_{\mathbf{k}}^{ \pm}=\frac{1}{2}\left\{\tilde{\epsilon}_{f, \mathbf{k}}+\epsilon_{\mathbf{k}} \pm\left[\left(\tilde{\epsilon}_{f, \mathbf{k}}-\epsilon_{\mathbf{k}}\right)^{2}+4 \tilde{V}^{2}\right]^{1 / 2}\right\} .
$$

These are the same bands as in the non-interacting case ( $U=0)$ except that the bare energies $\epsilon_{f}$ and $V$ are replaced by $\tilde{\epsilon}_{f, \mathbf{k}}$ and $\tilde{V}$, respectively. Neglecting the selfenergy's $k$ dependence, which is expected to be weak compared to the $\omega$ dependence, one finds for the quasiparticle effective mass at the Fermi energy

$$
\frac{m^{*}}{m}=1+\frac{V^{2}}{\left[\epsilon_{f}+\Sigma\left(\mathbf{k}_{F}, 0\right)\right]^{2}} \frac{1}{Z}
$$

The specific-heat coefficient and the spin susceptibility are found as

$$
\gamma=\frac{2 \pi^{2}}{3} \tilde{N}(0), \quad \chi=\frac{2 \mu_{m}^{2} \tilde{N}(0)}{1+F_{0}^{a}}
$$

where $\tilde{N}(0)$ is the renormalized total density of states at the Fermi level

$$
\tilde{N}(0)=N_{c}^{(0)}(0)+N_{f}^{(0)}(0) / Z
$$

with $N_{c, f}^{(0)}(0)$ the densities of states of conduction electrons and $f$ electrons, respectively, in the limit $U=0$. The factor $R=\left(1+F_{0}^{a}\right)^{-1}$, often called the generalized Wilson ratio, in $\chi(44)$ expresses the effect of quasiparticle interactions in terms of the Landau parameter $F_{0}^{a}$.

There are various approximation schemes available, allowing to estimate the parameters $Z, \tilde{\epsilon}_{f}$ in the above semi-phenomenological quasiparticle theory. The simplest one uses slave-boson mean-field theory, in the limit $U \rightarrow \infty$ (Newns and Read, 1987). One finds an approximate mapping to a model of two non-interacting, hybridizing fermion bands, with a quasiparticle dispersion given in (42). Assuming a flat conduction-band density of states $N_{c}^{(0)}(\epsilon)=1 /(2 D)$, and for $D \gg \epsilon_{f}, V$, the renormalized $f$ level $\tilde{\epsilon}_{f}$ is found as a solution of

$$
\epsilon_{f}-\tilde{\epsilon}_{f}=\frac{2}{\pi} \Gamma \ell n\left(\tilde{\epsilon}_{f} / D\right)
$$

which defines a characteristic energy scale, $T^{*}=\tilde{\epsilon}_{f}$, i.e., the distance of the renormalized $f$ level to the Fermi energy $\left(\Gamma=\pi N_{c}^{(0)}(0) V^{2}\right.$ is the bare hybridization width). The renormalized $f$-electron density of states can be expressed as

$$
\frac{1}{Z} N_{f}^{(0)}(0)=\frac{2}{\pi} \frac{\Gamma}{\tilde{\epsilon}_{f}} \frac{1}{T^{*}} .
$$

The specific heat and the spin susceptibility are given by (44), with the Landau parameter $F_{0}^{a}=0$. A finite value of $F_{0}^{a}$ can be obtained from the contribution of fluctuations about the mean field (Houghton, Read, and Won, 1988). If the $f$ states are sufficiently far below the Fermi level, the characteristic temperature assumes within this approximation the same functional dependence as the Kondo temperature of the single impurity problem:

$$
T^{*} \sim D \exp \left(-\frac{\pi\left|\epsilon_{f}\right|}{2 \Gamma}\right)
$$

where $T^{*} \ll D$, implying an exponentially small quasiparticle weight factor $Z \sim T^{*} / \Gamma \ll 1$. This is the regime of heavy fermion metals, with large effective mass ratio $m^{*} / m \gg 1$.

A similar mean-field approach can be taken to the Kondo lattice model (Burdin, Georges, and Grempel, 2000), showing that (at least) two energy scales are relevant for the Kondo lattice problem. The onset of Kondo screening upon lowering $T$ happens around $T_{\mathrm{K}}^{(1)}$, the (single-impurity) Kondo temperature, whereas the Fermi liquid is only established below $T_{\text {coh }}$. Typically, $T_{\text {coh }}<$ $T_{\mathrm{K}}^{(1)}$, leading to a crossover regime which is wider than in the single-impurity case (dubbed protracted screening). In the weak-coupling limit, both $T_{\mathrm{K}}^{(1)}$ and $T_{\text {coh }}$ can be obtained analytically, with the result:

$$
\begin{aligned}
& T_{\mathrm{K}}^{(1)}=D \exp \left(-\frac{1}{N_{0} J}\right) F_{K}\left(n_{c}\right), \\
& T_{\text {coh }}=D \exp \left(-\frac{1}{N_{0} J}\right) F_{\text {coh }}\left(n_{c}\right)
\end{aligned}
$$

where $F_{K}$ and $F_{\text {coh }}$ are functions of filling (and shape) of the conduction band, and $N_{0}$ is the conduction-band density of states for $J=0$. In this approximation the ratio $T_{\mathrm{coh}} / T_{\mathrm{K}}^{(1)}$ is a function of the conduction-band properties only, but is independent of the Kondo coupling $J$. 
Beyond slave bosons, the local correlation physics of Anderson and Kondo lattice model has been studied using the Dynamical Mean-Field Theory (DMFT). The DMFT makes use of the limit of infinite spatial dimensions (Metzner and Vollhardt, 1989), in which the selfenergy $\Sigma(\mathbf{k}, \omega)$ becomes independent of momentum $\mathbf{k}$. This corresponds to a mapping of the Anderson lattice model to an effective Anderson impurity model with energy-dependent hybridization function $\Delta(\omega)$ (Georges et al., 1996). The self-consistency equation relates the local $f$ Green's function of the lattice $G^{\text {loc }}$ to that of the effective impurity model $G^{\mathrm{SIAM}}$

$$
\begin{aligned}
G^{\mathrm{loc}}(z) & =\int d \epsilon \frac{N_{0}(\epsilon)}{z-\epsilon_{f}-\Sigma_{f}(z)-\frac{V^{2}}{z-\epsilon-\epsilon_{c}}} \\
& =\left[z-\epsilon_{f}-\Delta(z)-\Sigma_{f}(z)\right]^{-1}=G^{\mathrm{SIAM}}(z) .
\end{aligned}
$$

The effective Anderson model has been solved by Quantum Monte Carlo (QMC, see Georges et al. 1996) and Numerical Renormalization Group (NRG, see Bulla 1999) techniques. The most accurate study using NRG by Pruschke, Bulla, and Jarrell (2000) shows that near halffilling, $n_{c}=1$, the coherence temperature is actually larger than the single-impurity Kondo temperature, their ratio depending on the Kondo coupling $J$. More importantly, at lower fillings $n_{c} \lesssim 0.8$, the ratio $T_{\text {coh }} / T_{\mathrm{K}}^{(1)}$ was numerically found to be independent of $J$, and in the small- $n_{c}$ limit was proportional to $n_{c}$. These findings are in qualitative agreement with earlier QMC studies (Jarrell, 1995; Tahvildar-Zadeh, Jarrell, and Freericks, 1997).

The results for the $J$ dependence of the coherence scale of Burdin, Georges, and Grempel (2000) and Pruschke, Bulla, and Jarrell (2000), namely $T_{\text {coh }} \propto T_{\mathrm{K}}^{(1)}$, indicate that the so-called exhaustion scenario of Noziéres (1985), predicting $T_{\text {coh }} \propto\left(T_{\mathrm{K}}^{(1)}\right)^{2} / D$, is incorrect. As detailed by Noziéres (2005), the original exhaustion argument (Noziéres, 1985) is too simplistic, e.g., it does not correctly account for the flow of the Kondo coupling. We also note that the exhaustion scenario has been falsified experimentally: In $\mathrm{Ce}_{x} \mathrm{La}_{1-x} \mathrm{~Pb}_{3}$ Kondo behavior of single-impurity type has been observed down to lowest temperatures for $x$ up to $80 \%$, with $T_{\mathrm{K}}=3.3 \mathrm{~K}$ essentially independent of $x$ (Lin et al., 1987), whereas the exhaustion arguments would predict a suppression of $T_{\mathrm{K}}$ for $x>0.1 \%$.

The approximate treatments of the Anderson and Kondo lattice models discussed so far cannot capture the competing magnetic ordering tendencies arising from non-local inter-moment exchange. On the mean-field level, this competition can be included by decoupling a non-local interaction with suitable auxiliary fields (Iglesias, Lacroix, and Coqblin, 1997; Kiselev, Kikoin, and Oppermann, 2002; Senthil, Sachdev, and Vojta, 2003; Senthil, Vojta, and Sachdev, 2004). In addition, extensions of DMFT have been devised (Si et al., 2001, 2003). We will return to these aspects in Sec. III.I. We also note that non-local interactions may enhance $T_{\text {coh }}$ compared to $T_{\mathrm{K}}^{(1)}$ due to mutual screening of local moments. Ex- perimental indications for this have been seen, e.g., in $\mathrm{CeCoIn}_{5}$ by Nakatsuji et al. (2002), see Sec. IV.A.4.

\section{Multi-channel Kondo effect}

Nozières and Blandin (1980) realized the importance of the proper matching of the degrees of freedom of impurity and environment for local non-Fermi-liquid behavior in Kondo models. This can be discussed using a generalized Kondo model, where an impurity spin of size $S$ is coupled to several identical conduction bands (labeled $\alpha, \alpha=$ $1, \ldots ., M$, and called "channels"):

$$
H=\sum_{\mathbf{k}, \sigma, \alpha} \epsilon_{\mathbf{k}} c_{\mathbf{k} \sigma \alpha}^{\dagger} c_{\mathbf{k} \sigma \alpha}+J \mathbf{S} \cdot \sum_{\alpha} \mathbf{s}_{\alpha}
$$

where $\mathbf{s}_{\alpha}$ is the local conduction electron spin of band $\alpha$. The Hamiltonian (51) has a high degree of symmetry: besides spin rotation invariance there is separately invariance against unitary transformations in channel space.

For the low-energy behavior of (51) with $J>0$, three cases have to be distinguished: (i) $S=M / 2$, here the impurity spin is exactly screened by $M$ electrons of spin $1 / 2$, yielding local FL behavior; (ii) $S>M / 2$ yields an effective uncompensated spin of size $S-M / 2-$ this underscreened Kondo effect leads to non-analytic corrections to local FL behavior; (iii) $S<M / 2$ results in overscreening, associated with true local NFL behavior - this will be discussed in the following.

The occurrence of a new type of ground state for $S<M / 2$ can be understood as follows (Nozières and Blandin, 1980). Suppose $S=\frac{1}{2}$, and $M=2$; if the effective exchange coupling $j$ would scale to infinity as in the single-channel case considered in the last section, the impurity would bind one electron with opposite spin orientation in each of the two channels. The net spin projection of the overscreened impurity site would be $S_{z}^{\prime}=-S_{z}$ if $S_{z}$ is the momentary impurity spin projection. Now, virtual hopping processes of neighboring electrons (with spin projection $S_{z}$ due to the Pauli principle) induce an exchange interaction between the effective spin $\mathbf{S}^{\prime}$ and the neighboring electron spins, which is again antiferromagnetic. According to the initial assumption, this coupling scales to infinity; this process repeats itself and thus does not converge. One concludes that the fixed point at infinite coupling is not stable, i.e., there must exist a fixed point at some finite coupling strength. In the limit of large channel number $M$ the new fixed point is accessible by "poor man's scaling" (Nozières and Blandin, 1980). One finds that the $\beta$ function for the scale-dependent coupling $j$ now has a weak-coupling expansion:

$$
\beta(j)=j^{2}-\frac{M}{2} j^{3}+\ldots
$$

replacing (35). Thus there exists a fixed point $(\beta=0)$ at $j^{*}=N_{0} J^{*}=2 / M \ll 1$, within the range of validity of the expansion. 
At the new fixed point the impurity spin is not exactly screened, which destroys the Fermi liquid found in the exactly screened case, and leads to local non-Fermiliquid behavior. Exact treatments based upon the Bethe ansatz method (Andrei and Destri, 1984; Schlottmann and Sacramento, 1993; Tsvelik and Wiegmann, 1984) bosonization and conformal field theory (Affleck and Ludwig, 1991a,b) have been used to determine the limiting low-temperature behavior.

For the $M$-channel, spin $S=1 / 2$, Kondo model (51) the most striking result is a finite impurity entropy $S(T=$ 0 ) indicating some kind of fractional degeneracy of the ground state (Andrei and Destri, 1984),

$$
S(T=0)=\ln \left[2 \cos \left(\frac{\pi}{M+2}\right)\right] .
$$

The low-temperature specific heat and spin susceptibility in zero magnetic field follow the power-law behavior (Andrei and Destri, 1984; Tsvelik and Wiegmann, 1984)

$$
\frac{C_{v}^{\mathrm{imp}}}{T} \sim \chi_{\mathrm{imp}}(T) \sim T^{\frac{4}{2+M}-1}, M>2
$$

and a logarithmic law for $M=2$ (Schlottmann and Sacramento, 1993). The leading low-temperature powerlaw correction of the resistivity is found as

$$
\rho(T)-\rho_{0} \sim T^{\alpha_{\rho}}
$$

where $\alpha_{\rho}=\frac{2}{2+M}$ for $S=\frac{1}{2}$ and $M \geq 2$.

As noted by Nozières and Blandin (1980), perfect channel symmetry is rather unlikely due to anisotropies in realistic crystalline fields. The presence of channel asymmetry will always cause a flow to the singlechannel Kondo fixed point. Accidentally symmetrybreaking fields may be small: in such a situation, the multi-channel fixed point would dominate over a sizeable energy/temperature range, before branching off to a single-channel fixed point at some low energy scale.

An interesting route to two-channel Kondo behavior is the quadrupolar Kondo effect. Here, the impurity has a doubly degenerate non-magnetic ground state, and the role of the two internal states is taken by orbital degrees of freedom. Then, the conduction electron spin directions provide two independent screening channels, with channel symmetry being protected by spin symmetry.

To date, experimental realizations of multi-channel or quadrupolar Kondo physics in Kondo alloys have been elusive. For a comprehensive discussion of experimental candidate systems and a presentation of theoretical results available see Cox and Zawadowski (1998).

\section{Impurity quantum phase transitions}

Quantum impurity models can show phase transitions at zero temperature - these transitions are special cases of boundary QPT, with the impurity being a zerodimensional boundary of the system. At such a transition only the internal degrees of freedom of the impurity become critical. Impurity QPT are of current interest in diverse fields such as unconventional superconductors, quantum dot systems, quantum computing, and they will also play a central role in the scenario of so-called local quantum criticality in heavy-fermion systems, described in Sec. III.I.1 - for a review see Vojta (2006a). In the following, we briefly mention a few quantum impurity models which display QPT.

In general, Kondo-type models can feature a QCP between phases with quenched and unquenched (or partially quenched) impurity moment. A well-studied example is the pseudogap Kondo model, where the density of states of the conduction electrons vanishes as $|\omega|^{r}$ near the Fermi level. Here, no screening occurs for small Kondo coupling $J$, whereas the impurity spin is screened for large $J$. The critical behavior depends on the value of the exponent $r$ and on the presence or absence of particlehole symmetry. The pseudogap Kondo model is in particular relevant to impurity moments in $d$-wave superconductors where the exponent $r=1$ characterizes the density of states of the Bogoliubov quasiparticles. Interestingly, the behavior of the standard two-channel Kondo model, discussed in Sec. II.F.3, can also be understood in terms of an impurity QPT: tuning the ratio of the coupling strengths of the two channels drives the system from one Fermi-liquid phase to another, with the equalstrength two-channel situation point being the QCP.

A further class of models with impurity QPT are those where the impurity is coupled to a bosonic bath - this can represent spin, charge, or lattice collective modes of the environment. Bosonic impurity models have been first introduced for the description of dissipative dynamics in quantum systems (Leggett et al., 1987). The simplest realization is the so-called spin-boson model, describing a spin or two-level system linearly coupled to a bath of harmonic oscillators. This system has two phases: a delocalized phase with weak dissipation, where the spin tunnels between its two possible orientations, and a localized phase where large dissipation suppresses tunneling in the low-energy limit, leading to a doubly degenerate ground state with a trapped spin. As above, the universality class of the transition depends on the low-energy behavior of the bath spectral density. SU(2)-symmetric generalizations of the spin-boson model include so-called Bose Kondo models where an impurity spin is coupled to magnetic fluctuations of the bulk material.

In the particular context of strongly correlated electron systems, which often feature Fermi-liquid quasiparticles and strong spin fluctuations at the same time, the question of the interplay between fermionic and bosonic "Kondo" physics arises. This naturally leads to so-called Fermi-Bose Kondo models where an impurity spin is coupled to both a fermionic and a bosonic bath:

$$
H=\sum_{k \sigma} E_{k} c_{k \sigma}^{\dagger} c_{k \sigma}+J \mathbf{S} \cdot \mathbf{s}_{0}+H_{\phi}+\gamma_{0} \mathbf{S} \cdot \phi_{0} .
$$

Here, the fermionic bath is represented by the spin- $1 / 2$ electrons $c_{k \sigma}$, with local spin density $\mathbf{s}_{0}$, and $\phi$ is the 
spin-1 order-parameter field of the host magnet. Its dynamics, contained in $H_{\phi}$, is most naturally described by a quantum $\phi^{4}$ theory; under certain conditions this can be replaced by a model of free vector bosons.

Most interesting is the case of a bosonic bath with zero or small gap, corresponding to the vicinity to a magnetic QCP in the $d$-dimensional bulk. The RG analysis shows that the two baths compete: For large $J$ fermionic Kondo screening wins, resulting in a fully screened spin. Large $\gamma_{0}$ can completely suppress Kondo screening, driving the system into the intermediate-coupling fixed point of the Bose Kondo problem, corresponding to a bosonic fluctuating phase with universal local-moment correlations (Vojta, Buragohain, and Sachdev, 2000). The competition is captured by the RG equations (Sengupta, 2000; Si et al., 2001; Smith and Si, 1999)

$$
\begin{aligned}
& \beta(\gamma)=\frac{3-d}{2} \gamma-\gamma^{3}, \\
& \beta(j)=j^{2}-j \gamma^{2} .
\end{aligned}
$$

The phase diagram for the Bose-Fermi Kondo model thus shows a Kondo-screened phase, a bosonic fluctuating phase, and a continuous quantum phase transition in between. The suppression of Kondo screening by bulk spin fluctuations is of relevance in materials with strong magnetism, like cuprate superconductors, and near magnetic bulk QCP. A model of the form (56) also appears within extended DMFT, where a Kondo lattice model is mapped onto a Bose-Fermi Kondo model with additional self-consistency conditions, for details see Sec. III.I.1.

\section{G. Non-Fermi-liquid behavior from disorder}

The influence of disorder on metallic systems has a wide range of aspects, ranging from diffusive transport to Anderson localization, as reviewed by Lee and Ramakrishnan (1985). As discussed above, the (perfectly screened) Kondo effect leads to a local Fermi liquid around a magnetic impurity. Usually, in a random dilute solution of impurities, the microscopic parameters $J$ and $N_{0}$ which determine the coupling between magnetic impurity and conduction electrons, acquire well defined values yielding a unique Kondo temperature for the system under consideration. However, in disordered systems, a distribution of Kondo temperatures may arise from statistically fluctuating $J$ and/or $N_{0}$. This might occur in metals near the metal-insulator transition as exemplified by heavily doped semiconductors (Lakner et al., 1994; Paalanen and Bhatt, 1991; Paalanen et al., 1986; Sarachik, 1995), or by alloys with two different non-magnetic constituents leading to different local environments. A similar situation arises in disordered heavyfermion systems, where the competition of Kondo screening and interaction of magnetic moments takes place in a disordered environment, often leading to non-Fermiliquid behavior (Stewart, 2001, 2006).
That disorder can give rise to phases with anomalous behavior has been known for some time (McCoy and $\mathrm{Wu}, 1968)$. The behavior is not confined to a critical point in the phase diagram but may persist over an entire region. These so-called Griffiths-McCoy phases were discussed originally in disordered classical systems (Griffiths, 1969). Much stronger singularities are found in quantum models as has been established theoretically in recent years, see Sec. III.J.2.

The subject of NFL behavior driven by disorder has been reviewed recently by Miranda and Dobrosavljević (2005). It is therefore not necessary to give full coverage of this important aspect of NFL physics here. We will come back to this question in Sec. III.J where the effect of disorder on magnetic quantum phase transitions in metallic systems will be discussed.

\section{FERMI-LIQUID INSTABILITIES AT QUANTUM PHASE TRANSITIONS: THEORY}

A quantum system can undergo a continuous phase transition at $T=0$ upon variation of some non-thermal control parameter (Sachdev, 1999; Sondhi et al., 1997; Vojta, 2003a). Near the critical point of such a quantum phase transition in the itinerant electron systems of interest here, the finite-temperature behavior is characterized by scaling laws with temperature exponents different, in general, from those of Fermi-liquid theory. This may be considered as a breakdown of the Fermi-liquid state induced by quantum fluctuations near the critical point. As is the case with usual classical continuous phase transitions, the different systems fall into universality classes, depending on the symmetry properties of the phase transition. In contrast to classical phase transitions, also the dynamics affects critical thermodynamic properties, and therefore a larger number of different universality classes can be expected, e.g., depending on the presence or absence of an efficient coupling between the order parameter and fermionic quasiparticles.

\section{A. Classical vs. quantum phase transitions}

Phase transitions at $T=0$ are dominated by quantum effects, in contrast to classical phase transitions at $T>0$, even though both may occur in the same physical system. Any continuous finite-temperature phase transition is classical in the following sense: continuous phase transitions have divergent correlation length and time. The order parameter $\phi$ (magnetization, staggered magnetization, etc.) fluctuates coherently over increasing distances and time scales as one approaches the transition. The latter implies that there exists a characteristic frequency, $\omega_{\phi}$, for order-parameter fluctuations, which tends to zero at the transition. The system behaves classically (even if quantum effects are important at short length scales) if the transition temperature $T_{c}$ satisfies $k_{B} T_{c} \gg \hbar \omega_{\phi}$. This argument shows at once that quantum phase transitions, 
for which $T_{c}=0$, are qualitatively different: their critical fluctuations require a quantum-statistical description.

Let us start the discussion with the partition function $Z=\operatorname{Tr} \exp (-\beta H), \beta=1 / k_{B} T$. For a classical continuous phase transition, the partition function may be represented in terms of the relevant time-independent order-parameter field $\phi(\mathbf{r})$ as a functional integral over all configurations of $\phi$

$$
Z_{c l}=Z_{c l}^{0} \int \mathcal{D} \phi(\mathbf{r}) \exp \left(-\beta F_{c l}\{\phi\}\right)
$$

where $F_{c l}$ is the Landau-Ginzburg-Wilson (LGW) free energy functional

$$
\begin{aligned}
F_{c l}\{\phi\} & =\int d^{d} r \mathcal{F}_{c l}[\phi(\mathbf{r})] \\
& =\epsilon_{0} \int d^{d} r\left[\phi(\mathbf{r})\left(\delta_{0}+\xi_{0}^{2} \nabla^{2}\right) \phi(\mathbf{r})+u_{0} \phi^{4}(\mathbf{r})+\ldots\right]
\end{aligned}
$$

and $Z_{c l}^{0}$ is the partition function of the non-critical degrees of freedom, and $\epsilon_{0}$ is a microscopic energy scale. The parameter $\delta_{0}$ depends on temperature and tunes the system through the phase transition.

For the quantum system we can represent the partition function in a somewhat similar form, the difference being that the quantum nature of the order-parameter field requires that one keeps track of the order in which the $\phi$ operators appear. Realizing that $\exp (-\beta H)$ is a time evolution operator on the imaginary time axis, the partition function $Z(\beta)$ may be represented as a path integral over all configurations $\phi(\mathbf{r}, \tau)$ with $\phi(\mathbf{r}, 0)=\phi(\mathbf{r}, \hbar \beta)$ :

$$
Z(\beta)=Z_{0} \int \mathcal{D} \phi(\mathbf{r}, \tau) \exp (-S\{\phi\})
$$

where $S$ is the Euclidian action,

$$
S\{\phi\}=\int_{0}^{\hbar \beta} d \tau \int d^{d} r \mathcal{L}\{\phi(\mathbf{r}, \tau)\}
$$

with the Lagrange density $\mathcal{L}$ given by the LGW free energy expression amended by the kinetic energy of quantum fluctuations of the order-parameter field, $\mathcal{L}_{k i n}$,

$$
\mathcal{L}=\mathcal{L}_{k i n}+\mathcal{F}_{c l}[\phi(\mathbf{r}, \tau)]
$$

The actual form of $\mathcal{L}_{\text {kin }}$ depends on the dynamics of the system. We note that these general arguments, however, do not exclude the possibility that $\mathcal{L}$ is a highly non-local, non-linear object, such that an efficient description of the critical behavior in terms of an order-parameter field $\phi(\mathbf{r}, \tau)$ is not any more useful. We will defer a discussion of these complications, which arise e.g. in ferromagnetic metals, to Sec. III.H.

If the dependence of $\phi(\mathbf{r}, \tau)$ on $\tau$ can be neglected, as is the case at a finite- $T$ phase transition, the contribution from quantum fluctuations $\mathcal{L}_{\text {kin }} \rightarrow 0$, and the $\tau$-integral in (61) gives back the factor of $\beta$ in the expression (58) for the classical partition function. This can be seen more clearly in the Fourier representation, where $S$, e.g., in the case of an insulating magnet, takes the form

$$
S=\frac{1}{\beta V} \sum_{\mathbf{k} \omega_{n}} \phi_{-\mathbf{k},-\omega_{n}}\left[\epsilon_{0}\left(\delta_{0}+\xi_{0}^{2} k^{2}\right)+\omega_{n}^{2}\right] \phi_{\mathbf{k}, \omega_{n}}+S_{4}
$$

with $\omega_{n}=2 \pi n k_{B} T$ the (imaginary) frequency and $\mathbf{k}$ the wavevector of the order-parameter fluctuation. The term $S_{4}$ stands for the fourth-order term in the LGW function. The non-thermal control parameter $\delta_{0}$ acquires a $T$-dependent renormalization from the interactions; the transition occurs at $\delta_{0}=\delta_{c}$ where the renormalized $\delta$ vanishes. In the following we will denote the distance to the quantum critical point (QCP) by

$$
r=\delta_{0}-\delta_{c}(T=0),
$$

which can be tuned by varying pressure [then $r=(p-$ $\left.p_{c}\right) / p_{c}$ ], magnetic field, or chemical composition.

Upon approaching a finite-temperature phase transition, the energy of the characteristic order-parameter fluctuations $\omega_{\phi}$ (proportional to the renormalized value of $\delta$ ) becomes eventually smaller than $k_{B} T$. Then only the $\omega_{n}=0$ term in (63) contributes to the critical behavior, which is thus governed by spatial order-parameter fluctuations only, Eq. (59). In contrast, at $T=0$ temporal fluctuations are not negligible, but appear to be intimately intertwined with spatial fluctuations. The representation $(60,61)$ of the partition function suggests that the system behaves like a $(d+1)$-dimensional classical system, which is anisotropic since the "gradient energy" in the time direction may be of different order (e.g. first order, see below) as in the spatial directions (usually second order), and the time axis is restricted to the interval $\hbar \beta$. The anisotropy of the fictitious classical system may be characterized by the so-called dynamical exponent $z$, defined by the scaling of frequency with wavevector $\omega \sim k^{z}$. For the LGW function (63), $\omega$ scales as $k^{1}$ and hence the bare value of $z=1$ in this case. For metallic magnets we will have $z>1$. (Interactions may change the bare value of $z$ below the upper critical dimension of a field theory.)

The effective dimensionality for a system near a quantum phase transition is thus $d_{\text {eff }}=d+z$. In many cases $d_{\text {eff }}$ is equal to or larger than the upper critical dimension, $d_{c}^{+}$, of the respective field theory; $d_{c}^{+}=4$ for the magnetic transitions to be discussed below. While a phase transition with $d_{\mathrm{eff}}<d_{c}^{+}$is controlled by an interacting fixed point and usually obeys strong hyperscaling properties, a transition with $d_{\mathrm{eff}} \geq d_{c}^{+}$can be described within mean-field theory, and hyperscaling is violated due to the presence of dangerously irrelevant operators.

\section{B. Scaling properties near quantum phase transitions}

The functional integral formulation allows one to employ the well-established picture of scaling near a continuous phase transition. As such a transition is approached, 
both the order-parameter correlation length $\xi$ and correlation time $\xi_{\tau}$ (i.e., the correlation length along the imaginary time axis) diverge:

$$
\xi \sim|r|^{-\nu}, \quad \xi_{\tau} \sim \xi^{z}
$$

where $r$ (64) measures the distance to the QCP. The correlation-length exponent $\nu$ of the quantum transition is different from the one of a possible finite-temperature transition at $T_{c}$ in the same system (which describes the divergence $\xi \sim\left|T-T_{c}\right|^{-\nu_{c l}}$ ). At finite temperature, the $(d+z)$-dimensional quantum system has a finite length in the time direction, $L_{\tau}=\hbar \beta$ (61). Its properties can then be deduced from finite-size scaling (Privman, 1990).

We are now in the position to discuss the properties of a system near a quantum critical point, located at $T=0$, $r=0$ (Fig. 2). The QCP is usually the endpoint of a line of continuous finite- $T$ transitions. (Exceptions are lowdimensional systems where order at finite $T$ is prohibited by the Mermin-Wagner theorem, or systems where no order parameter can be defined for $T>0$, as is the case for metal-insulator transitions or transitions in the topology of the Fermi surface.) In general, the boundary of the ordered phase follows $T_{c} \propto(-r)^{\psi}$ where $\psi$ is the so-called shift exponent. In the immediate vicinity of this boundary there is a region of classical non-Gaussian criticality. The disordered phase of the system at finite $T$ can be divided into distinct regimes: For low $T$ and $r>0$ thermal effects are negligible $\left(L_{\tau} \gg \xi_{\tau}\right.$, equivalently $T \ll r^{\nu z}$ ), and the critical singularity is cutoff by the deviation of the control parameter $r$ from criticality. This regime is dubbed "quantum disordered" and characterized by well-defined quasiparticle excitations; for a magnetic transition in a metallic system this will be the usual Fermi-liquid regime. For $r<0$ and $T>T_{c}$, but still $L_{\tau} \gg \xi_{\tau}$, we are in the "thermally disordered" regime; here the order is destroyed by thermal fluctuations of the ordered state (yet quasiparticles are still well defined on intermediate scales). A completely different regime is the high-temperature regime above the QCP where $\xi_{\tau} \gg L_{\tau}$. In this "quantum critical" regime, bounded by crossover lines $T \sim|r|^{\nu z}$, the critical singularity is cutoff by the finite temperature. The properties are determined by the unconventional excitation spectrum of the quantum critical ground state, where the quasiparticles of the stable phases are replaced by a critical continuum of excitations. In the quantum critical regime, this continuum is thermally excited, resulting in unconventional power-law temperature dependencies of physical observables.

Assuming that the critical behavior is governed by $\xi$ and $\xi_{\tau}$, the critical contribution to the free energy density, $f_{\text {cr }}=f-f_{\text {reg }}$, should follow the homogeneity law

$$
f_{\mathrm{cr}}(r, T)=b^{-(d+z)} f_{\mathrm{cr}}\left(r b^{1 / \nu}, T b^{z}\right)
$$

where $b$ is an arbitrary scale factor. Note that this "naive scaling" (equivalent to hyperscaling) is valid only below the upper critical dimension, $d_{\mathrm{eff}}<d_{c}^{+}$, and we will comment on deviations later on. Choosing $b=\xi$, Eq. (66)

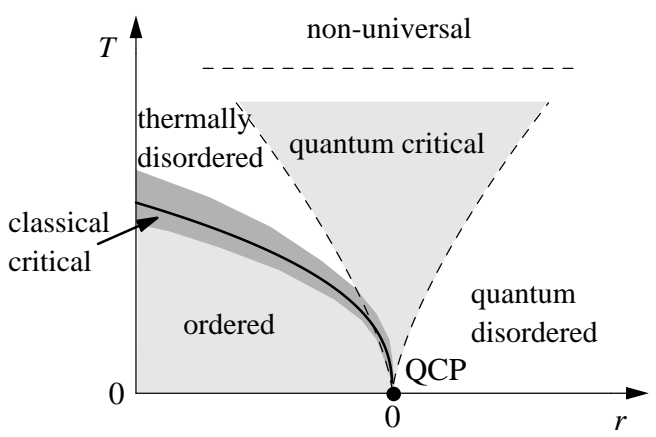

FIG. 2 Generic phase diagram in the vicinity of a continuous quantum phase transition. The horizontal axis represents the control parameter $r$ used to tune the system through the QPT, the vertical axis is the temperature $T$. The dashed lines indicate the boundaries of the quantum critical region. The lower crossover lines are given by $T \propto|r|^{\nu z}$; the hightemperature crossover to non-universal (lattice) physics occurs when the correlation length is no longer large to microscopic length scales. The solid line marks the finitetemperature boundary between the ordered and disordered phases. Close to this line, the critical behavior is classical.

can be cast into the scaling form $f_{\mathrm{cr}}=\xi^{-(d+z)} \phi_{1}\left(\xi_{\tau} / L_{\tau}\right)$, or, equivalently, the ansatz:

$$
\begin{aligned}
f_{\text {cr }} & =\rho_{0} r^{\nu(d+z)} \phi_{2}\left(\frac{T}{r^{\nu z}}\right) \\
& =\rho_{0} T^{(d+z) / z} \phi_{3}\left(\frac{r}{T^{1 /(\nu z)}}\right),
\end{aligned}
$$

where $T$ is measured in units of $T_{0}, T_{0}$ and $\rho_{0}$ being nonuniversal constants, while $\phi_{1,2,3}(x)$ are universal scaling functions.

From Eq. (67) we can immediately deduce the critical contribution to the specific heat, $C=T \partial S / \partial T$, as

$$
C_{\mathrm{cr}}(r=0, T) \propto T^{d / z}
$$

in the quantum critical regime. If the quantum disordered regime of Fig. 2 is a Fermi liquid, then Eq. (67) yields for its specific-heat coefficient $C_{\mathrm{cr}} / T(T \rightarrow 0) \propto$ $r^{\nu(d-z)}$.

As is clear from Fig. 2, a quantum critical point can be generically approached in two different ways: $r \rightarrow 0$ at $T=0$ or $T \rightarrow 0$ at $r=0$. The power-law behavior of physical observables in both cases can often be related. Let us discuss this idea by looking at the entropy $S$. It goes to zero at the QCP (exceptions are impurity transitions discussed in Sec. II.F.4 and by Vojta, 2006a), but its derivatives are singular. The specific heat $C$ will show power-law behavior, as does the observable $B=\partial S / \partial r$. At a pressure-tuned phase transition, $r=\left(p-p_{c}\right) / p_{c}, B$ measures the thermal expansion,

$$
\alpha=\left.\frac{1}{V} \frac{\partial V}{\partial T}\right|_{p}=-\left.\frac{1}{V} \frac{\partial S}{\partial p}\right|_{T} .
$$


$B / C$ defines the Grüneisen parameter $\Gamma$,

$$
\Gamma=\frac{\alpha}{C_{p}}=-\frac{1}{V_{m} T} \frac{(\partial S / \partial p)_{T}}{(\partial S / \partial T)_{p}}
$$

where $V_{m}=V / N$ the molar volume. Taking the ratio of the singular parts of $B$ and $C$ one observes that the scaling dimensions of $T$ and $S$ cancel, and therefore $B / C$ scales as the inverse of the tuning parameter $r$. Thus, one obtains a universal divergence in the low- $T$ limit (Zhu et al., 2003)

$$
\begin{aligned}
& \Gamma_{\mathrm{cr}}(T=0, r)=\frac{B_{\mathrm{cr}}}{C_{\mathrm{cr}}}=G_{r}|r|^{-1} \\
& \Gamma_{\mathrm{cr}}(T, r=0)=G_{T} T^{-1 /(\nu z)},
\end{aligned}
$$

With the help of the scaling ansatz (67) the full scaling form of $\Gamma$ can be determined, for details see Zhu et al. (2003). Remarkably, in the $T \rightarrow 0$ limit, even the prefactor $G_{r}$ is universal and given by a combination of critical exponents. Further we note that $\Gamma$ does not diverge at a finite- $T$ phase transition, thus a divergence of $\Gamma$ is a unique signature of a continuous QPT.

If the control parameter of the QPT is not pressure but an external magnetic field $H$, the quantity $B$ is the $T$-derivative of the magnetization $M$, and the role of the Grüneisen ratio is played by

$$
\Gamma_{H}=-\frac{(\partial M / \partial T)_{H}}{c_{H}}=-\frac{1}{T} \frac{(\partial S / \partial H)_{T}}{(\partial S / \partial T)_{H}}=\left.\frac{1}{T} \frac{\partial T}{\partial H}\right|_{S}
$$

It can be determined directly from the magnetocaloric effect by measuring the change of temperature in response to an adiabatic $(S=$ const) change of $H$.

As the scaling arguments can be invalid above the upper critical dimension, we will quote concrete results for the critical points of metallic magnets in Sec. III.D.

We finally turn to dynamical scaling. Any physical quantity depending on $\mathbf{r}$ and $t$ (or equivalently $\mathbf{k}$ and $\omega$ ) in the critical region close to the QPT (but sufficiently far from the associated finite- $T$ transition) should depend on space and time only through the scaled variables $k \xi$ and $\omega \xi_{\tau}$, since $\xi$ is the only length scale and $\xi_{\tau}$ is the only time scale in that regime. (Note that multiple time scales may be present in a multi-component system, see the discussion in Sec. III.H.1.) The Fourier components of a physical quantity $X$ affected by the transition are thus expected to exhibit the following scaling behavior

$$
\begin{aligned}
X(k, \omega ; r, T) & =\xi^{d_{x}} F_{x}\left(k \xi, \omega \xi_{\tau}, \xi_{\tau} / L_{\tau}\right) \\
& =T^{-d_{x} / z} \tilde{F}_{x}\left(\frac{k^{z}}{T}, \frac{\omega}{T}, \frac{T}{r^{\nu z}}\right)
\end{aligned}
$$

where $d_{x}$ is the scaling dimension of the observable $X$. Exactly at the quantum critical point this reduces to

$$
X(k, \omega ; r=0, T=0)=k^{-d_{x}} F_{x}^{*}\left(k^{z} / \omega\right)
$$

We again note that all scaling relations are only expected to be valid if the critical point satisfies hyperscaling properties, which is true below the upper critical dimension $d_{c}^{+}$. Scaling above $d_{c}^{+}$in the presence of a dangerously irrelevant variable will be briefly discussed in Sec. III.D.

\section{Itinerant fermion systems}

Quantum phase transitions in itinerant electron systems were first studied in a pioneering paper by Hertz (1976). Hertz pointed out that near a phase transition at $T=0$ static and dynamic properties are inextricably mixed and applied a renormalization group (RG) treatment to model systems of this type. This work was later reconsidered and extended by Millis (1993).

\section{Definition of the Hertz model}

In the context of strongly correlated electron systems one is mainly interested in magnetic phase transitions in metals. As prototypes we will consider ferromagnetic $(\mathrm{FM})$ and antiferromagnetic (AFM) phase transitions. We will assume the collective behavior near the transition to be characterized by a real $N$-component orderparameter field $\phi$, representing the magnetization (for the FM) or the staggered magnetization (for the AFM). A number of simplifications occur in the limit $N \rightarrow \infty$, although the actual number of components is $N \leq 3$. The effective action may be derived from the Hamiltonian either by introducing the collective field in functional integral representation and integrating out the electron degrees of freedom (Hertz, 1976), or by more conventional techniques (Moriya, 1985). Assuming that the resulting action $S\{\phi\}$ can be expanded in powers of $\phi$ with spatially local coefficients, one arrives at the Hertz model,

$$
S=S_{2}+S_{4}+\ldots .
$$

Here the second-order term is given by

$$
S_{2}=\frac{1}{\beta V} \sum_{\mathbf{k}, \omega_{n}} \epsilon_{0}\left[\delta_{0}+\xi_{0}^{2} k^{2}+\frac{\left|\omega_{n}\right|}{\gamma(k)}\right] \phi_{\mathbf{k}, \omega_{n}} \cdot \phi_{-\mathbf{k},-\omega_{n}}
$$

where the prefactor of $\phi^{2}$ is nothing but the inverse of the dynamical spin susceptibility $\chi^{-1}\left(\mathbf{k}, \omega_{n}\right)$. In this case the microscopic correlation length $\xi_{0}$ is $\sim k_{F}^{-1}$, where $k_{F}$ is a Fermi wavevector, and $\epsilon_{0}$ is the microscopic energy scale, given by the Fermi energy $\epsilon_{F}$. The momentum summation extends up to a (bare) cutoff $\Lambda_{0}$.

The dynamic contribution $\left|\omega_{n}\right| / \gamma(k)$ accounts for damping of the spin fluctuations $\phi_{\mathbf{k}, \omega_{n}}$ by particle-hole pairs excited across the Fermi level ("Landau damping"). Their phase space increases linear with $\omega$. For a ferromagnetic transition (or other transitions with a $Q=0$ order parameter), $\gamma(k)=v_{F} k$ as $k \rightarrow 0$, i.e., the damping rate diverges due to the abundance of particle-hole pairs with small momentum. This results in a theory with (bare) dynamical exponent $z=3$. For an antiferromagnetic transition $\gamma(k) \sim \gamma_{0}$, independent of $k$, yielding $z=2$. These forms of $\gamma(k)$ hold if the wavevector of the spin mode in either case is well inside the particlehole continuum, i.e., if the ordering wavevector $\mathbf{Q}$ connects points on a $(d-2)$-dimensional manifold of points on the Fermi surface. For an antiferromagnetic system 
with a small Fermi volume and a large ordering vector $Q>2 k_{F}$, the particle-hole pairs decouple from the spin fluctuations and $\omega$ enters quadratically as in (63). The crossover from linear to quadratic $\omega$ dependence has been discussed in detail by Sachdev, Chubukov, and Sokol (1995); Sachdev (1999). The special situation where an antiferromagnetic mode is tied to wavevector $2 k_{F}$ at the edge of the particle-hole continuum ("nesting") will be considered separately in Sec. III.H.5.

The fourth-order term $S_{4}$ of the action accounts for the self-interaction of spin excitations,

$$
S_{4}=u_{0} \int d \tau \int d^{d} r\left[\phi(r, \tau)^{2}\right]^{2}
$$

with $u_{0}$ denoting the strength of the interaction.

Let us point out here that the damping term in the Hertz theory has been derived under the assumption of Fermi-liquid behavior of the electronic quasiparticles. This needs to be justified a posteriori and is discussed in Sec. III.H. We also note that in the ordered phase, i.e., $r<0, T<T_{c}$ in Fig. 3, the action (78) does not apply: the form of the damping term will be modified due to the appearance of a gap in the electronic band structure. (This is already clear from the Goldstone theorem which requires the mode damping to vanish as $k \rightarrow 0$ in the ordered phase.) Technically, the limit of vanishing order parameter, $\langle\phi\rangle \rightarrow 0$, does not commute with the long-distance expansion, $k, \omega \rightarrow 0$. A discussion of the field theory in the ordered state can be found, e.g., in Sachdev, Chubukov, and Sokol (1995).

\section{Pressure vs. field tuning}

Frequently, antiferromagnetic critical points are accessed by tuning pressure or magnetic field. The presence of a magnetic field changes the universality class of the system, as its presence breaks time-reversal invariance which leads to a different dynamics of the order parameter. Provided that the system has spin rotation invariance perpendicular to the field, a finite uniform magnetization leads to a precession of the AFM order parameter described by an additional term in the action,

$$
S_{\mathrm{pr}}=\int d \tau \int d^{d} r \mathbf{b} \cdot i\left(\phi \times \partial_{\tau} \phi\right)
$$

where $\mathbf{b}$ is the effective exchange field parallel to the magnetization. As this term changes the dynamics, it affects the quantum critical behavior. This effect is most drastic in an insulator (or an itinerant AFM with $Q>2 k_{F}$ ) where the dynamics arises from a term of the form $\iint\left(\partial_{\tau} \phi\right)^{2}$ in the absence of magnetic fields. Therefore the dynamical critical exponent is given by $z=1$ for $\mathbf{b}=0$. In contrast, Eq. (80) implies $z=2$, and the QCP has the same critical properties as the superfluid quantum phase transition of bosons driven by a change of the chemical potential.
In itinerant magnets, the precession term $i \omega_{n} \phi_{x} \phi_{y}$ competes with the Landau damping $\left|\omega_{n}\right| \phi^{2}$, which both have the same scaling dimension. Technically, the term in Eq. (80) is an exactly marginal perturbation, and critical properties depend on the exact ratio of precession and Landau damping, see Fischer and Rosch (2005) for an extensive discussion. (Experimentally, many systems have a strong Ising anisotropy due to spin-orbit interactions, rendering the precession term irrelevant.)

Besides suppressing antiferromagnetism, a uniform field can also induce large non-analytic changes in the uniform magnetization. These phenomena, usually occurring in almost ferromagnetic systems, are referred to as metamagnetism. Frequently, metamagnetic transitions, being not associated with a symmetry change of the system, are of first order at low $T$ and feature a finitetemperature critical endpoint. However, by utilizing additional tuning parameters, the critical endpoint may be suppressed down to $T=0$, resulting in a quantum critical endpoint. Such a scenario has been proposed for the bilayer ruthenate $\mathrm{Sr}_{3} \mathrm{Ru}_{2} \mathrm{O}_{7}$ (Grigera et al., 2001). The theoretical description starts out from the Hertz model (77) for a ferromagnetic order parameter, supplemented by a sixth-order term in $\phi$. The resulting phase transition is qualitatively similar to a Hertz-type Ising transition in a system with $z=3$; for a detailed discussion we refer the reader to Millis et al. (2002).

\section{Scaling equations}

The model defined by the action $(77,78)$ has been studied near its critical point (Hertz, 1976; Millis, 1993) using the perturbative RG. To define a RG transformation one investigates how a change of the cutoff (either in momentum or frequency space or both) and their subsequent rescaling can be absorbed in a redefinition of the coupling constants, using for example the perturbative expressions for the free energy (Millis, 1993). Following Millis (1993), we use a scheme where simultaneously the cutoffs in momentum space, $\Lambda_{k} \rightarrow \Lambda_{k} / b$, and frequency space, $\Lambda_{\omega} \rightarrow \Lambda_{\omega} / b^{z}$, are reduced. The change of $\delta$, $u$, the dimensionless temperature $\mathcal{T}$, and the dimensionsless free energy density $\mathcal{F}=F \xi_{0}^{d} /\left(T_{0} V\right)$ under infinitesimal RG transformations are given by (Millis, 1993):

$$
\begin{aligned}
\frac{d \mathcal{T}}{d \ell n b} & =z \mathcal{T}, \\
\frac{d \delta}{d \ell n b} & =2 \delta+4(N+2) u f_{2}(\mathcal{T}, \delta), \\
\frac{d u}{d \ln b} & =(4-d-z) u-4(N+8) u^{2} f_{4}(\mathcal{T}, \delta), \\
\frac{d \mathcal{F}}{d \ell n b} & =(d+z) \mathcal{F}-\frac{N}{2} f_{0}(\mathcal{T}, \delta)
\end{aligned}
$$

with the initial conditions $\mathcal{T}=T, \delta=\delta_{0}, u=u_{0}, \mathcal{F}=0$. While these equations differ in prefactors from the ones of Millis (1993), the derivation of the $f_{i}$ terms can be found there. Briefly, the $f_{i}$ are explicit functions of $\mathcal{T}$, 


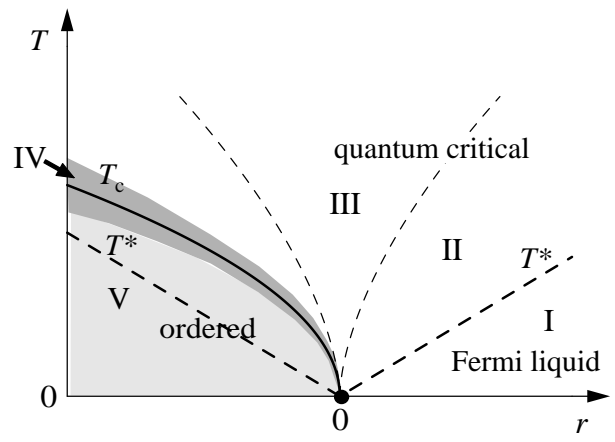

FIG. 3 Phase diagram of the Hertz model. I: Fermi-liquid regime; II/III: Quantum critical regime; IV: Non-Gaussian classical critical regime; V: Magnetically ordered phase. The regimes II and III are distinguished in the behavior of the correlation length $\xi$, see (97). The quantum critical regime also extends into the ordered phase, with singular behavior for $T>T^{*}$ similar to regime II. Note that transport properties may show more complicated crossovers, see Sec. III.F.

$\delta$, and the bare cutoff $\Lambda_{0}$ (which we will set to unity in the following). The dependence of the number of orderparameter components $N$ can be absorbed by defining $\bar{f}_{0}=N f_{0} / 2, \bar{f}_{2}=4(N+2) f_{2}, \bar{f}_{4}=4(N+8) f_{4}$. At low $T$ close to the critical point, $\delta=0$, we have

$$
\begin{aligned}
& \bar{f}_{0}(\mathcal{T})=\bar{f}_{0}(0)+\frac{4}{3} \pi K_{d}\left[\mathcal{T}^{2}-\frac{4 \pi^{2}}{15} \mathcal{T}^{4}\right]+\ldots \\
& \bar{f}_{2}(\mathcal{T})=\bar{f}_{2}(0)+B \mathcal{T}^{2}+\ldots
\end{aligned}
$$

with $K_{d}=\left(2^{d-1} \pi^{d / 2} \Gamma(d / 2)\right)^{-1}=1 / 2 \pi^{d-1}, d=2,3$. At high $T$, i.e., in the quantum critical regime,

$$
\bar{f}_{0}(\mathcal{T})=D \mathcal{T}, \quad \bar{f}_{2}(\mathcal{T})=C \mathcal{T}
$$

where $D, C$ are constants of order unity. $\bar{f}_{4}$ does not have any critical dependence on $\mathcal{T}$ or $\delta$ and may be replaced by a (positive) constant.

The RG equations (81) have a Gaussian fixed point at $\mathcal{T}=u=\delta=0$, which is unstable with respect to the tuning parameter $\delta$. Of particular interest is the differential equation (81c) for the quartic coupling $u$ : if $d+z>4, u$ scales to zero, i.e., the upper critical dimension is given by $d_{c}^{+}=4-z$ rather than $d_{c}^{+}=4$ as for classical critical phenomena. Thus in many cases of interest one can expect the critical behavior to be that of the Gaussian model. For example, in the cases considered above, $z=2$ (antiferromagnetic metal) or $z=3$ (ferromagnetic metal), and in dimensions $d \geq 2$ the Gaussian model applies (the case $d=z=2$ is marginal and needs special consideration).

\section{Solution for $d+z>4$}

We will now discuss the results obtained by solving Eqs. (81). Interpreting $b$ as a flow variable, straight- forward integration, employing the Gaussian approximation, yields the scale-dependent quantities

$$
\begin{aligned}
\mathcal{T}(b) & =T b^{z} \\
u(b) & =u_{0} b^{4-d-z} \\
\delta(b) & =b^{2}\left[\delta_{0}+u_{0} \int_{1}^{b} d b_{1} b_{1}^{1-(d+z)} \bar{f}_{2}\left(T b_{1}^{z}\right)\right] .
\end{aligned}
$$

Under the RG process the scale $b$ increases and the system moves away from criticality, i.e., $\delta(b)$ increases, until $\delta(b)=1$ is reached at $b=b_{0}$. The regimes I and II/III in Fig. 3 are distinguished according to whether the renormalized temperature is smaller or larger than the cutoff in energy, $\mathcal{T}\left(b_{0}\right) \gtrless 1$.

The condition $\mathcal{T}\left(b_{0}\right) \ll 1$ defines the quantum disordered (or Fermi-liquid) regime (Hertz, 1976). To obtain the limits of this regime, one may put $\mathcal{T}=0$ in the equation for $\delta(b)$, to determine $b_{0}$ from $\delta\left(b_{0}\right)=1$. From $\mathcal{T}\left(b_{0}\right) \ll 1$ one finds with the aid of $(84)$

$$
T \ll|r|^{z \nu},
$$

where $\nu=1 / 2$ is the Gaussian correlation-length exponent (for $d+z \geq 4$ ), and $r$ (64) is the distance to the QCP as above. In lowest order perturbation theory we have $r=\delta_{r}$ with the renormalized control parameter

$$
\delta_{r}=\delta_{0}+\frac{u \bar{f}_{2}(0)}{z+d-2}
$$

and $\delta_{r} \geq 0$ is required here. The relation $T^{*} \sim|r|^{z \nu}$ marks the crossover line from the quantum disordered regime to the quantum critical regime. (We note that a line $T^{*} \sim|r|^{z \nu}$ also exists inside the ordered phase, Fig. 3, with singular contributions to thermodynamics and transport above $T^{*}$.)

In the opposite case, when $\mathcal{T}\left(b_{0}\right) \gg 1$ at $\delta\left(b_{0}\right) \sim 1$, it is convenient to perform the scaling successively in the regimes $\mathcal{T}(b) \ll 1$ and $\mathcal{T}(b) \gg 1$. Starting at small scales the results derived above may be used to determine $\delta(b)$ and $u(b)$ at the scale $b_{1}$ where $\mathcal{T}\left(b_{1}\right) \sim 1$ and hence $b_{1}=$ $T^{-1 / z}$. The result is

$$
\begin{aligned}
& \delta_{1}=\delta\left(b_{1}\right)=T^{-2 / z}\left[\delta_{r}+B u_{0} T^{(d+z-2) / z}\right], \\
& u_{1}=u\left(b_{1}\right)=u_{0} T^{(d+z-4) / z} .
\end{aligned}
$$

These values provide the initial conditions for the scaling in the regime where $\mathcal{T}(b) \gg 1$ and $\bar{f}_{2}(\mathcal{T}) \simeq C \mathcal{T}$. With the new scaling variable $v=u \mathcal{T}$ one can decouple the flow of $\mathcal{T}$ and $v$

$$
\frac{d \delta}{d \ell n b}=2 \delta+C v, \quad \frac{d v}{d \ell n b}=(4-d) v .
$$

Integration of these equations starting from the initial conditions $v_{1}=u_{1} \mathcal{T}\left(b_{1}\right)=u_{1}, \delta_{1}$ yields for dimensions $d>2$ (assuming $d+z>4$ ),

$$
\begin{aligned}
& v(b)=v_{1}\left(b / b_{1}\right)^{4-d}, \\
& \delta(b)=\delta_{1}\left(b / b_{1}\right)^{2}+\left[\left(b / b_{1}\right)^{2}-\left(b / b_{1}\right)^{4-d}\right] \frac{C v_{1}}{d-2} .
\end{aligned}
$$


In $d>2$ dimensions, one finds therefore

$$
\delta(b)=b^{2}\left[\delta_{r}+\left(B+\frac{C}{d-2}\right) u_{0} T^{1 / \psi}\right]
$$

where the so-called shift exponent

$$
\psi=\frac{z}{d+z-2}
$$

describes the position of the finite-temperature phase transition (see below). For $d=2$ (and $z>2$ ) logarithmic terms appear in the solution for $\delta(b)$ :

$$
\left.\delta(b)=\left(b / b_{1}\right)^{2}\left[\delta_{1}+\ln \left(b / b_{1}\right) C v_{1}\right)\right]
$$

For dimensions $2 \leq d<4$, both $v(b)$ and $\delta(b)$ increase as the scaling proceeds and $b$ is growing. If $\delta\left(b_{0}\right) \sim 1$ is reached when $v\left(b_{0}\right) \ll 1$, the Gaussian approximation used here is sufficient. If, however, $v(b)$ becomes of order unity while $\delta(b)$ is still small, the scaling leaves the weak-coupling regime and crosses over to a new regime characterized by non-Gaussian behavior. The condition for Gaussian behavior, the so-called Ginzburg criterion, is thus $v\left(b_{0}\right) \ll 1$, or more explicitly, in $d=3$ dimensions,

$$
v\left(b_{0}\right)=\frac{u T}{\left[\delta_{r}+(B+C) u T^{1 / \psi}\right]^{1 / 2}} \ll 1
$$

correcting the exponent in the numerator of Millis (1993). The condition is violated within a narrow strip around the temperature for which the denominator in (93) vanishes. One can therefore locate the transition temperature $T_{c}(\delta, b)$ from the condition $v\left(b_{0}\right)=\mathcal{O}(1)$ which for all $z>1$ leads to

$$
T_{c} \simeq \epsilon_{0}\left[\frac{-\delta_{r}}{(B+C) u}\right]^{\psi}
$$

for $r=\delta_{r}<0$, see Fig. 3). In (94) we have reinstated the microscopic energy scale $\epsilon_{0}$, to indicate the real temperature scale. From Eq. (91) we see that in $d=3$ the transition temperature varies as $T_{c} \propto(-r)^{2 / 3}$ and $(-r)^{3 / 4}$ for antiferromagnets and ferromagnets, respectively; the $d=2$ result is in Eq. (95) below.

The width of the critical region around $T_{c}$, where the Gaussian approximation is expected to fail, can be obtained from $v\left(b_{0}\right)=1$. For $d+z>4$, as assumed, the width of the critical region is seen to shrink to zero as $T_{c} \rightarrow 0$, thus validating (94).

In two dimensions, the presence or absence of the phase transition depends on the symmetry of the order parameter. The Ginzburg criterion can be used to locate the boundary of the regime where both the correlation length is large and the coupling strong. For Ising (XY) symmetry this will give an estimate for the phase transition (Kosterlitz-Thouless) temperature $T_{c}$. Defining $b^{*}$ by the Ginzburg condition $v\left(b^{*}\right)=\mathcal{O}(1)$ we obtain from $\delta\left(b^{*}\right)=1$ for $T_{c}$ in $d=2, z>2$

$$
T_{c} \sim \frac{-\delta_{r}}{u\left\{1+B+C \ell n\left[1 /\left(\sqrt{u} T_{c}^{(z-2) / 2 z}\right)\right]\right\}} .
$$

Up to a logarithmic correction, the transition temperature in $d=2$ is linear in $(-r)$.

The correlation length $\xi$ of the order-parameter fluctuations is given by $\delta\left(b_{0}\right) \sim\left(b_{0} / \xi\right)^{2}$. In the quantum disordered regime, $T \ll|r|^{z \nu}$, the correlation length is determined by the distance to criticality,

$$
\xi=|r|^{-\nu},
$$

with $\nu=1 / 2$ and $r=\delta_{r}$ (86). In the quantum critical regime, $T \gg|r|^{z \nu}$, one finds for $d=3$ :

$$
\xi^{-2}=\delta_{r}+(B+C) u T^{1 / \psi}
$$

where the first (second) term dominates in regime II (III), see Fig. 3. Note that all prefactors in Eqs. (90-97) which involve both $B$ and $C$ are not exact, as they neglect corrections of order unity which arise from the crossover regime, where $T(b) \approx 1$. Including this intermediate regime one obtains for $d>2$ (Garst, 2003)

$$
\xi^{-2}=\delta_{r}+4(N+2) \frac{K_{d} \Gamma\left(\frac{z+d-2}{z}\right) \zeta\left(\frac{z+d-2}{z}\right)}{z \cos \left(\frac{d-2}{2 z} \pi\right)} u T^{1 / \Psi} .
$$

For $d=2, z=3$, Eq. (97) is replaced by

$$
\xi^{-2}=\delta_{r}+\left(B+C \ln \frac{b_{0}}{b_{1}}\right) u T
$$

with $b_{1}=T^{-1 / 2}$ and $b_{0}$ determined from $\delta\left(b_{0}\right)=1$ and (92).

\section{Solution for $d+z=4$}

The marginal case $d=z=2$ requires special consideration, as in this case logarithmic corrections to the Gaussian behavior appear. The scaling of the interaction $u$ is now governed by the second term in Eq. (81c). Integrating (81c) taking only the leading constant term of $\bar{f}_{4}$ into account gives

$$
u(b)=\frac{u_{0}}{1+u_{0} \bar{f}_{4} \ell n b}
$$

For large $b$ (i.e. large $b_{0}$, at the endpoint of the scaling process), the scaled interaction is seen to be independent of $u_{0}$, and decreases $\sim 1 / \ell n b$.

Next, integrating the scaling equation (81b) for $\delta(b)$ using (84a) and the scaled interaction (100), one finds

$$
\delta(b)=b^{2} \delta_{r}+\beta(b, T) T^{2}
$$

where the renormalized control parameter $\delta_{r}$ is given by

$$
\delta_{r}=\delta_{0}+u_{0} \bar{f}_{2}(0) \int_{0}^{\infty} d \ell n b_{1} \frac{e^{-2 \ell n b_{1}}}{1+u_{0} \bar{f}_{4} \ell n b_{1}}
$$

and $\beta(b, T)$ can be expressed in a power series in $T^{2}$.

The quantum disordered regime is again defined by the condition $\mathcal{T}\left(b_{0}\right) \ll 1$ at $\delta\left(b_{0}\right) \sim 1$. Observing that 
$\delta\left(b_{0}\right)=b_{0}^{2} \delta_{r}$ in that regime, the boundary of the quantum critical regime is given by $T \sim \delta_{r}$. In the quantum critical regime, $T \gg \delta_{r}$, the scaling up to $b \sim b_{T}=T^{-1 / 2}$, where $\mathcal{T}\left(b_{T}\right) \sim 1$ yields (with $G=$ const)

$$
\begin{aligned}
& \delta_{1} \equiv \delta\left(b_{T}\right)=\frac{\delta_{r}}{T}+\frac{G}{\ell n \frac{1}{T}} \\
& u_{1} \equiv u\left(b_{T}\right)=\frac{2}{\bar{f}_{4} \ell n \frac{1}{T}} \ll 1 .
\end{aligned}
$$

instead of Eqs. (87) found in the case $d+z>4$.

The correlation length in the quantum disordered regime is still given by Eq. (96), while in the quantum critical regime we have

$$
\xi^{-2} \sim \delta_{r}\left(\ln \frac{c}{\delta_{r}}\right)^{-\frac{N+2}{N+8}}+T \frac{\ln \ln (\bar{c} / T)}{\ln (\bar{c} / T)},
$$

where the first (second) term dominates in regime II (III).

It should be emphasized that the expansion in regime III is not very revealing as it is only valid if $\ln \ell n(\bar{c} / T) \gg$ 1. Obviously, and as pointed out by Sachdev and Dunkel (2006), this condition will never be satisfied in practice. Thus, the $d=z=2$ quantum critical theory is in general not in a weak-coupling regime at any $T>0$. Instead, a strongly coupled effective classical model emerges which can be used to determine the critical dynamics, for details see Sachdev and Dunkel (2006). However, in the context of metallic antiferromagnets of interest here, even more serious complications arise, which we discuss in Sec. III.H.2.

\section{Thermodynamic quantities}

As shown above, in the cases where $d+z>4$ the system scales to the non-interacting (Gaussian) fixed point. Although the quartic coupling $u$ is formally irrelevant, it still affects a number of physical quantities, like the location of the critical line or the order-parameter susceptibility at $r=0, T>0$. To describe this within a scaling approach, as in Sec. III.B, one has to include explicitly the quartic coupling $u$ in the scaling ansatz for the free energy

$$
f_{\mathrm{cr}}=\rho_{0} T^{(d+z) / z} \phi_{4}\left(\frac{r}{T^{1 /(\nu z)}}, u T^{(d+z-4) / z}\right)
$$

which replaces Eq. (67). While $u$ is irrelevant, it has to be kept as $\phi_{4}$ can become a singular function of $u$. In such a case the "naive" scaling relations derived from Eq. (67) are modified, and $u$ is called "dangerously irrelevant".

The free energy in the theory of Hertz is obtained by integrating the RG equation (81d) for the free-energy density up to the scale where $\delta\left(b_{0}\right)=1$ and adding the Gaussian free energy

$$
\begin{aligned}
F_{G}(b)= & -\int_{0}^{1} d^{d} k \int_{0}^{\gamma(k)} \frac{d \epsilon}{\pi} \operatorname{coth}\left(\frac{\epsilon}{2 T(b)}\right) \\
& \times \arctan \left[\frac{\epsilon}{\gamma(k)\left[\delta(b)+k^{2}\right]}\right]
\end{aligned}
$$

\begin{tabular}{ccccc} 
& $d=2$ & $d=3$ & $d=2$ & $d=3$ \\
$z=2$ & $z=2$ & $z=3$ & $z=3$ \\
\hline & & & & \\
$\alpha_{\mathrm{cr}} \sim$ & $\operatorname{Tr}^{-1}$ & $\operatorname{Tr}^{-1 / 2}$ & $\operatorname{Tr}^{-3 / 2}$ & $\operatorname{Tr}^{-1}$ \\
& & & & \\
$C_{\mathrm{cr}} \sim \quad$ & $T \ell n \frac{1}{r}$ & $-T r^{1 / 2}$ & $\operatorname{Tr}^{-1 / 2}$ & $\operatorname{T\ell n} \frac{1}{r}$ \\
$\Gamma_{r, \mathrm{cr}}=$ & $\left(r \ln \frac{1}{r}\right)^{-1}$ & $-(2 r)^{-1}$ & $(2 r)^{-1}$ & $\left(r \ln \frac{1}{r}\right)^{-1}$
\end{tabular}

TABLE I Results for thermal expansion $\alpha$ (69), specific heat $C$, and Grüneisen ratio $\Gamma(70)$, at a $\mathrm{QCP}$ in the quantum disordered (Fermi-liquid) regime $T \ll r^{z / 2}$. The left columns $(z=2)$ are for a metallic AFM, the right ones $(z=3)$ for a metallic FM. Non-universal prefactors of $\alpha_{\mathrm{cr}}$ and $C_{\mathrm{cr}}$ are not shown. The prefactors of $\Gamma_{\mathrm{cr}}$ and $\Gamma_{H, \mathrm{cr}}$ are (up to the logarithmic correction for $d=z$ ) universal; these quantities are given by $\Gamma_{\mathrm{cr}}=(d r / d p) \Gamma_{r, \mathrm{cr}} / V_{m}$ for pressure-tuned QCP with $r=\left(p-p_{c}\right) / p_{c}$, and $\Gamma_{H, \mathrm{cr}}=(d r / d H) \Gamma_{r, \mathrm{cr}}$ for fieldtuned QCP with $r=\left(H-H_{c}\right) / H_{c}$. Note that for $d=3, z=$ 2 the specific heat is dominated by a non-critical fermionic contribution $C \sim T$ (Zhu et al., 2003).

at this scale. The Gaussian contribution to the free energy at the scale $b_{0}$ is then $F_{G}=b_{0}^{-(d+z)} F_{G}\left(b_{0}\right)$. The scale-dependent correction to the free energy, arising in the scaling process, is obtained by integrating the scaling equation (81d) for $\mathcal{F}(b)$ up to $b_{0}$ :

$$
F_{S C}=\int_{0}^{\ell n b_{0}} d \ell n b_{1} b_{1}^{-(d+z)} f_{0}\left(T\left(b_{1}\right)\right)
$$

Depending on the values of $d$ and $z$, different terms will dominate the critical contribution to the free energy; for details see Zhu et al. (2003).

In Tables I and II we present the results for the critical contributions to the specific heat $C$, the thermal expansion $\alpha$, Eq. (69), and the resulting Grüneisen ratio $\Gamma$, Eq. (70), derived from the free energy $F=F_{G}+F_{S C}$, in

\begin{tabular}{|c|c|c|c|c|}
\hline & $\begin{array}{l}d=2 \\
z=2\end{array}$ & $\begin{array}{l}d=3 \\
z=2\end{array}$ & $\begin{array}{l}d=2 \\
z=3\end{array}$ & $\begin{array}{l}d=3 \\
z=3\end{array}$ \\
\hline$\alpha_{\mathrm{cr}} \sim$ & $\ln \ln \frac{1}{T}$ & $T^{1 / 2}$ & $\ln \frac{1}{T}$ & $T^{1 / 3}$ \\
\hline$C_{\text {cr }} \sim$ & $T \ell n \frac{1}{T}$ & $-T^{3 / 2}$ & $T^{2 / 3}$ & $T \ell n \frac{1}{T}$ \\
\hline$\Gamma_{r, \mathrm{cr}} \sim$ & $\frac{\ln \ell n \frac{1}{T}}{T \ell n \frac{1}{T}}$ & $-T^{-1}$ & $-2 / 3 \ell n$ & $\left./ 3 \ln \frac{1}{T}\right)$ \\
\hline
\end{tabular}
both the quantum disordered and the quantum critical regimes. Up to logarithmic corrections the results agree with those derived from scaling in Sec. III.B. Note that for $d=z$ the prefactor in (71) vanishes. The $1 / r$ dependence of $\alpha_{\text {cr }}$ for $d=z$ arises from a $T^{2} \ell n 1 / r$ correction to $F_{\text {cr }}$ not captured by scaling. For the quantum critical

TABLE II Results for LGW QCP in the quantum critical regime $T \gg|r|^{z / 2}$ (cf. Table I). 
regime in $d=1 / \nu=2$ the thermal expansion is logarithmic. The argument of the logarithm is a power of $T$ for $d+z>4$ and is itself logarithmically dependent on $T$ for $d+z=4$; these features reflect the dangerously irrelevant or marginal nature of the quartic coupling $u_{0}$.

In addition to the critical contributions, the measured quantities also contain non-critical background components. We list here the full results for the purpose of comparisons with experiments in heavy-fermion compounds undergoing an antiferromagnetic transition $(z=2)$. Consider first $d=3$. At the QCP $(r=0)$

$$
\alpha=a_{1} T^{1 / 2}+a_{2} T
$$

where the $a_{2}$ term comes from the (fermionic) background contribution. However, approaching the QCP in the Fermi-liquid regime gives:

$$
\alpha=\left(a_{1} / r^{1 / 2}+a_{2}\right) T .
$$

For $d=2$ and $z=2$, we have at the QCP $(r=0)$

$$
\alpha=a_{1} \ln \left[b \ln \frac{T_{0}}{T}\right]+a_{2} T,
$$

and in the Fermi-liquid regime approaching the QCP:

$$
\alpha=\left(a_{1} / r+a_{2}\right) T \text {. }
$$

In two dimensions, the thermal expansion coefficient at $r=0$ diverges in the $T=0$ limit, Eq. (111), in sharp contrast to the textbook statement that $\alpha(T \rightarrow 0)=0$.

Finally, we turn to other thermodynamic quantities. The static susceptibility for a ferromagnet is $\chi \sim \xi^{2}$. However, $\chi$ is not critical in the case of an antiferromagnet and therefore the calculation is delicate. Ioffe and Millis (1995) performed a careful calculation of the various contributions and concluded that in $d=2$ (with $z=2$ ), the leading low- $T$ dependence of the susceptibility is given by $\chi \approx \chi_{0}-D T$ with non-universal constants $\chi_{0}$ and $D$ (probably positive for typical band structures). In analogy, we expect $\chi \approx \chi_{0}-D^{\prime} T^{3 / 2}$ in $d=3$.

For a QPT driven by a magnetic field $B$ (see Sec. III.C.2), however, the susceptibility, $\chi=\partial M / \partial B=$ $-\partial^{2} F / \partial B^{2}$, is more singular since the uniform magnetic field is a relevant perturbation acting as the control parameter, $r \propto B-B_{c}$. Below the upper critical dimension one obtains from scaling $\chi \propto T^{(d+z-2 / \nu) / z}$ in the quantum critical regime. For an itinerant magnet, $d=3, z=2$, Fischer and Rosch (2005) found in contrast that the critical contribution $\chi_{c}$ of $\chi$ is a singular function of the irrelevant coupling $u, \chi_{c} \sim \xi(T) T \sim T^{1 / 4} / \sqrt{u}$. It therefore strongly violates scaling.

\section{E. Self-consistent spin-fluctuation theories}

Our discussion in Secs. III.C and III.D was based on a renormalization group analysis following the work of Hertz (1976). However, many of the main results, e.g., for the phase diagram and susceptibility of nearly FM (Moriya and Kawabata, 1973) or AFM metals (Hasegawa and Moriya, 1974) in three dimensions, have been obtained earlier by Moriya and coworkers.

Their so-called SCR (self-consistently renormalized) formalism, described in detail by Moriya (1985), is a self-consistent one-loop approximation for the scattering of spin fluctuations (79). Within the RG approach one can understand the enormous success of SCR theory and where it fails. Technically, one obtains the SCR result from the RG equations (81) by neglecting the $u^{2}$ renormalizations in Eq. (81c) and by replacing the running $\delta(b)$ on the right-hand side of Eq. (81c) self-consistently by $b^{2} \lim _{b \rightarrow \infty} \delta(b) / b^{2}$. The SCR formalism works above the upper critical dimension, especially for $d=3$ and $z=2$ or 3 ; these approximations reproduce correctly the leading behavior of the relevant physical quantities including prefactors [e.g. of the correlation length, Eq. (98), contrary to claims of Millis (1993)]. The SCR formalism fails upon approaching the classical transition as it is blind for the Ginzburg criterion. A recent extension of the SCR method (Moriya, 2006) has been used to obtain the correct behavior at the upper critical dimension, $d+z=4$.

The importance of the SCR theory lies in its impressive success in quantitatively describing a large number of different $f$ - and $d$-electron systems (Lonzarich and Taillefer, 1985; Moriya, 1985; Moriya and Takimoto, 1995), predicting, e.g., transition temperatures by using parameters obtained from neutron scattering. It is therefore often the method of choice to fit experiments, in order to find out whether they are described by weakly interacting spin fluctuations. Here it turned out to be useful to describe the interaction of the spin fluctuations not by a $\phi^{4}$ term as above, but instead in the spirit of a non-linear sigma model - by a constraint set by the sum rule for the dynamic susceptibility $\left\langle S_{\alpha}^{2}\right\rangle=(2 \pi)^{-1} \sum_{\mathbf{q}} \int d \omega \operatorname{coth}(\omega / 2 T) \operatorname{Im} \chi_{\alpha \alpha}(\mathbf{q}, \omega)$ where $\mathbf{S}$ is the spin within the unit cell (Kambe et al., 1996; Lonzarich and Taillefer, 1985; Moriya, 1985; Moriya and Takimoto, 1995). Together with a parameterization of $\chi$ as in Eq. (78), this constraint can be used to determine the $T$ dependence of the renormalized mass $\delta$.

It would be worthwhile to develop the SCR theory further for the ordered side of the phase diagram, where in our opinion the formalism (Lonzarich and Taillefer, 1985; Moriya, 1985) is not as well justified as on the paramagnetic side.

\section{F. Transport properties}

For quantum critical systems above the upper critical dimension, the low- $T$ thermodynamics can be calculated reliably using standard methods like RG (see Sec. III.D). Much less is known about transport properties. Earlier predictions of the resistivity close to an antiferromagnetic QCP (see Moriya 1985 and references therein), for example, are not valid for perfectly clean (Hlubina and Rice, 
1995) or weakly disordered samples (Rosch, 1999, 2000). The difficulty arises because scattering from AFM spin waves is extremely anisotropic and affects only a small fraction of the Fermi surface. Therefore the transport properties depend both qualitatively and quantitatively on how other scattering mechanisms re-distribute quasiparticles and scatter them into these small regions.

In $d=3$ and for small static electric fields, it is possible to treat transport within a simple Boltzmann approach, as the QCP is above its upper critical dimension, spin- spin interactions are irrelevant in the RG sense, and because the concept of quasiparticles is still (marginally) valid in three dimensions. In $d=2$, a quasiparticle description is not possible at the QCP, see Sec. III.H.3, and a transport theory is more complicated (Kontani, Kanki, and Ueda, 1999). In the linear-response regime in $d=3$, the quasiparticle distribution $f_{\mathbf{k}}=f_{\mathbf{k}}^{0}-\Phi_{\mathbf{k}}\left(\partial f_{\mathbf{k}}^{0} / \partial \epsilon_{\mathbf{k}}\right)$ is linearized around the Fermi distribution $f_{\mathbf{k}}^{0}$ and the collision term reads (Hlubina and Rice, 1995)

$$
\left.\frac{\partial f_{\mathbf{k}}}{\partial t}\right|_{\text {coll }}=\sum_{\mathbf{k}^{\prime}} \frac{f_{\mathbf{k}^{\prime}}^{0}\left(1-f_{\mathbf{k}}^{0}\right)}{T}\left(\Phi_{\mathbf{k}}-\Phi_{\mathbf{k}^{\prime}}\right)\left[g_{\mathrm{imp}}^{2} \delta\left(\epsilon_{\mathbf{k}}-\epsilon_{\mathbf{k}^{\prime}}\right)+\frac{2 g_{S}^{2}}{\Gamma} n_{\epsilon_{\mathbf{k}}-\epsilon_{\mathbf{k}^{\prime}}}^{0} \operatorname{Im} \chi_{\mathbf{k}-\mathbf{k}^{\prime}}\left(\epsilon_{\mathbf{k}}-\epsilon_{\mathbf{k}^{\prime}}\right)\right]
$$

Here $g_{\text {imp }}^{2}$ and $g_{S}^{2}$ are transition rates for impurity scattering and inelastic scattering from spin fluctuations, described by the susceptibility $\chi_{\mathbf{q}}(\omega)$, and $f_{k}^{0}$ and $n_{\omega}^{0}$ are the Fermi and Bose functions, respectively. In the derivation of (113) one has assumed that the spin fluctuations stay in equilibrium, i.e., drag effects are neglected. This approximation implicitly assumes the presence of sufficient momentum relaxation, e.g., by strong Umklapp scattering. While this approximation gives probably at least qualitatively correct results in the case of a nearly AFM metal with a large Fermi volume, it is less clear whether it is valid for a FM where small momentum scattering dominates. The linearized Boltzmann equations in the presence of electric and magnetic fields, $\mathbf{E}$ and $\mathbf{B}$, can be written in the following form:

$$
\begin{aligned}
& \mathbf{v}_{\mathbf{k}} \mathbf{E}+\left(\mathbf{v}_{\mathbf{k}} \times \mathbf{B}\right) \partial_{\mathbf{k}} \Phi_{\mathbf{k}}=\iint \frac{d \mathbf{k}^{\prime} F_{\mathbf{k k}^{\prime}}}{\left|v_{\mathbf{k}^{\prime}}\right|(2 \pi)^{3}}\left(\Phi_{\mathbf{k}}-\Phi_{\mathbf{k}^{\prime}}\right),(114) \\
& F_{\mathbf{k k}^{\prime}}=g_{\mathrm{imp}}^{2}+\frac{2 g_{S}^{2}}{\Gamma T} \int_{0}^{\infty} d \omega \omega n_{\omega}^{0}\left[n_{\omega}^{0}+1\right] \operatorname{Im} \chi_{\mathbf{k}-\mathbf{k}^{\prime}}(\omega)
\end{aligned}
$$

where an integration over energy $\epsilon_{k^{\prime}}$ has been performed and all $\mathbf{k}$-vectors, Fermi velocities $\mathbf{v}_{\mathbf{k}}$ and integrations are restricted to the Fermi surface, using $\iint d \mathbf{k} /\left|v_{\mathbf{k}}\right| \equiv$ $\int d^{d} \mathbf{k} \delta\left(\epsilon_{\mathbf{k}}-\mu\right)$. Currents are calculated from $j^{i}=$ $\iint v_{\mathbf{k}}^{i} \Phi_{\mathbf{k}} d \mathbf{k} /\left[\left|v_{\mathbf{k}}\right|(2 \pi)^{3}\right]$.

If quasiparticles are scattered almost equally strongly all over the Fermi surface, the $\mathbf{k}$ dependence of $\Phi_{\mathbf{k}}$ is for $\mathbf{B}=0$ given by $\Phi_{\mathbf{k}} \propto \mathbf{E v}_{\mathbf{k}}$. Only under these assumptions, one recovers results for the resistivity at the QCP which have been derived many years ago by Mathon (1968); Moriya (1985); Ueda (1977).

$$
\begin{array}{r}
\rho \approx\left(\iint \frac{d \mathbf{k}}{\left|v_{\mathbf{k}}\right|} \iint \frac{d \mathbf{k}^{\prime}}{\left|v_{\mathbf{k}^{\prime}}\right|} F_{\mathbf{k k}^{\prime}}\left(\mathbf{v}_{\mathbf{k}} \hat{\mathbf{n}}-\mathbf{v}_{\mathbf{k}^{\prime}} \hat{\mathbf{n}}\right)^{2}\right) \\
\times\left(\iint \frac{d \mathbf{k}}{\left|v_{\mathbf{k}}\right|}\left(\mathbf{v}_{\mathbf{k}} \hat{\mathbf{n}}\right)^{2}\right)^{-2}
\end{array}
$$

where $\hat{\mathbf{n}}$ is a unit vector parallel to electric field and current. An almost equivalent form to (115) (including fac- tors of $\left.e^{2}, \hbar\right)$ can be found in Moriya (1985). As (115) is valid only for approximately uniform scattering, it can be used in the case of a FM QCP or if the scattering is dominated by (short-range) impurities. In the quantum critical region, $T>T^{*}$, of a QCP in $d=3$ one obtains (Mathon, 1968; Moriya, 1985; Moriya and Takimoto, 1995; Ueda, 1977)

$$
\Delta \rho \sim \begin{cases}T^{3 / 2} & \text { AFM disorder-dominated, } \Delta \rho \ll \rho_{0} \\ T^{5 / 3} & \text { FM }\end{cases}
$$

Eq. (115) fails completely in the case of an AFM QCP in a weakly disordered metal where quasiparticles scatter strongly close to lines on the Fermi surface with $\epsilon_{\mathbf{k}}=\epsilon_{\mathbf{k}+\mathbf{Q}}$, the so-called "hot lines". This has been first realized by Hlubina and Rice (1995), who argued that in a clean metal close to an AFM QCP, the resistivity is dominated by quasiparticles from regions of the Fermi surface far away from the hot lines, where scattering rates are proportional to $T^{2}$. Accordingly, $\rho \propto T^{2}$ is expected in an ultra-clean metal close to the AFM QCP. This effect can be understood in a simple relaxationtime approximation, where the resistivity is calculated from a Fermi-surface average of the $\mathbf{k}$-dependent scattering time, $\rho \propto 1 /\left\langle\tau_{\mathbf{k}}\right\rangle_{F S}$. Clearly, the longest scattering times dominate the resistivity and short-circuit contributions from the hot lines where $\tau$ is small. This effect is missed in (115), where $1 /\left\langle\tau_{\mathbf{k}}\right\rangle_{F S}$ is effectively replaced by $\left\langle 1 / \tau_{\mathbf{k}}\right\rangle_{F S}$. However, the predicted $T^{2}$ dependence of $\rho$ is unobservable in real systems, as tiny amounts of disorder change the picture qualitatively - this has been pointed out by Rosch $(1999,2000)$. Rosch (2000) solved the Boltzmann equation (114) numerically and analytically (in a certain scaling limit). For sufficiently small magnetic fields, weak disorder, and close to an AFM QCP in the paramagnetic phase in $d>2$, the temperaturedependent part of the resistivity, $\Delta \rho=\rho(T)-\rho_{0}$, obeys a scaling form as function of $B$, residual resistivity $\rho_{0}$, 
and distance from the QCP, here denoted as $r>0$ :

$$
\Delta \rho(T) \sim T^{d / 2} f\left[\frac{T^{(d-1) / 2}}{\rho_{0}}, \frac{r^{(d-1) / 2}}{\rho_{0}}, \frac{B}{\rho_{0} \sqrt{T}}\right] .
$$

Most interesting is the case $B=0, r=0$ where the scaling function crosses over from $f(\alpha, 0,0) \sim$ const for lowest temperature, i.e. $\alpha \rightarrow 0$, to $f(\alpha, 0,0) \sim \alpha^{-(4-d) /(5-d)}$ for temperatures above a crossover scale proportional to $\rho_{0}^{2 /(d-1)}$. Various crossovers in $d=3$ are discussed below in Eq. (118) for $B=0$. The rather complicated magnetic field dependence can be found in Rosch (2000).

Rosch (2000) has argued that the magneto-resistivity and the sensitivity to disorder can be used to decide whether an observed NFL behavior arises only from some "hot lines" on the Fermi surface typical for a spin-density wave transition or from a breakdown of the Fermi liquid on the full Fermi surface which is expected in some other scenarios (see Sec. III.H). Sensitivity to weak disorder and large non-linear effects in the magneto-resistivity are characteristic for the Hertz scenario.

For $B=0$ and $d=3$, one obtains for clean samples, characterized by a large residual resistivity ratio $[\mathrm{RRR}$, defined as $\left.\rho(T=300 \mathrm{~K}) / \rho_{0}\right]$,

$$
\frac{\Delta \rho(T)}{\rho\left(T_{0}\right)} \sim \begin{cases}t \sqrt{x} & , \max [x, \sqrt{r x}]<t<\sqrt{x} \\ t^{3 / 2} & , r<t<x \\ t^{2} / \sqrt{r} & , t<\min [r, \sqrt{x r}]\end{cases}
$$

replacing (115). The dimensionless temperature, disorder and distance from the critical point are defined by $t \sim T / T_{0}, x \sim \rho_{0} / \rho\left(T_{0}\right) \sim 1 / \mathrm{RRR}, r \sim 1 /\left(k_{F} \xi\right)^{2} \propto p-p_{c}$ (note $z \nu=1$ here). $T_{0}$ is a characteristic temperature scale, e.g., an effective Kondo temperature of a heavyfermion system. In a sufficiently clean sample with a $\mathrm{RRR}$ of the order of 100, the resistivity at the QCP is linear in temperature over a large regime $T_{0} / \mathrm{RRR} \lesssim T \lesssim$ $T_{0} / \sqrt{\mathrm{RRR}}$ due to the interplay of weak impurity scattering all over the Fermi surface and strong inelastic scattering close to the hot lines. Even at some distance from the QCP, one has to go to rather low temperatures to recover Fermi-liquid behavior. In a very clean sample with a large RRR, the crossover temperature $t \sim \sqrt{r x}$ can be considerably lower than the characteristic scale $(t \sim r)$ below which Fermi-liquid behavior is recovered in thermodynamic measurements.

The picture developed above, fits well to some experimental trends. In a large number of systems, the resistivity at the AFM QCP seems to rise with $T^{1.5}$, e.g. in $\mathrm{CeCu}_{2} \mathrm{Si}_{2}$ (Gegenwart et al., 1998), $\mathrm{CeNi}_{2} \mathrm{Ga}_{2}$ (Hauser et al., 1998), $\mathrm{CeCu}_{6-x} \mathrm{Ag}_{x}$ (Heuser et al., 1998a) $\mathrm{CeNi}_{2} \mathrm{Ge}_{2}$ (Grosche et al., 2000), $\mathrm{CePd}_{2} \mathrm{Si}_{2}$, or $\mathrm{CeIn}_{3}$ (Julian et al., 1996). All of these systems are "dirty" in the sense that $\Delta \rho \ll \rho_{0}$ in the temperature range where the above exponents have been fitted. In a few systems, the Lonzarich group (Grosche et al., 2000; Julian et al., 1996; Mathur et al., 1998) succeeded in preparing high-quality samples with RRR values of the order of 100 and the resistivity seems to rise with exponents smaller than 1.5. In the cleanest samples the resistivity is almost linear in $T$ over a substantial temperature range. Especially, the sensitivity to weak disorder might be interpreted as a signature that the systems can be described within the theory sketched above. Also, resistivity measurements experiments in $\mathrm{U}_{2} \mathrm{Pt}_{2} \mathrm{In}$ and $\mathrm{U}_{3} \mathrm{Ni}_{3} \mathrm{Sn}_{4}$ (Estrela et al., 2001a,b) have been fitted to the theory (118). For further comparison of theory and experiment and a detailed calculation of magneto-transport see Rosch (2000). A number of systems do not fit into the scenario above described, an example being $\mathrm{CeCu}_{6-x} \mathrm{Au}_{x}$ where $\Delta \rho$ is linear in $T$ in a regime with $\Delta \rho \ll \rho_{0}$. Remarkably, one obtains a linear $T$ dependence from (115) when one assumes that $3 \mathrm{~d}$ electrons scatter from 2d spin fluctuations (Rosch et al., 1997) - see Sec. IV.A.1 for a more detailed discussion.

\section{G. Approach to the QCP from the Fermi-liquid regime}

In the previous sections we used a description of QCP in terms of a bosonic order-parameter field (coupled to weakly interacting fermions) and identified the bosonic fluctuations as the origin of the singular behavior of thermodynamic and transport quantities. However, away from the QCP the system is a Fermi liquid in the low$T$ limit and therefore the approach to QCP for $T \rightarrow 0$ can alternatively be described by Fermi-liquid theory as mentioned in Sec. II.C. Within this complementary (but equivalent) language, the singularities in thermodynamics, for example, are not associated with bosonic fluctuations, but arise from the mass renormalization of the quasiparticles. One should keep in mind that Fermi liquid theory is devised to account rigorously for any lowenergy excitations, but cannot be used to calculate, e.g., short range properties such as the momentum dependence of susceptibilities.

We investigate the Fermi-liquid description of a QCP in the case of a Pomeranchuk instability (c.f. Sec. II.C). For such a uniform instability (at wavevector $\mathbf{Q}=0$ ) the momentum dependence of the quasiparticle properties is very weak, simplifying the description considerably.

We note that "hidden" order caused by a Pomeranchuk instability has attracted considerable interest recently, e.g., to explain the enigmatic ordering transition in $\mathrm{URu}_{2} \mathrm{Si}_{2}$ (Varma and Zhu, 2006), and in the context of the cuprate superconductors. Several calculations for the Hubbard model (Grote, Körding, and Wegner, 2002; Halboth and Metzner, 2000; Neumayr and Metzner, 2003), and the $t-J$ model (Yamase and Kohno, 2000) indicate a Pomeranchuk instability in the spin-symmetric $d$-wave channel. Pomeranchuk phases in isotropic Fermi liquids have been discussed by Oganesyan, Kivelson, and Fradkin (2001). The relation to nematic liquid crystals has been pointed out (Kivelson, Fradkin, and Emery, 1998).

In three dimensions in channels with even angular momentum, the Pomeranchuk instability is generically of first order due to the presence of cubic terms in the 
Ginzburg-Landau theory. It has been argued that even in $d=2$ strong fluctuations may drive the transition first order, thus avoiding critical quantum fluctuations (Kee, Kim, and Chung, 2003; Khavkine et al., 2004). Full quantum critical behavior is restored in these $2 \mathrm{~d}$ models, if a sufficiently strong repulsive term is added to the forward-scattering interaction (Yamase, Oganesyan, and Metzner, 2005).

In the neighborhood of a Pomeranchuk instability the electron system shows unusual properties due to a "soft" Fermi surface, leading to a strongly enhanced decay rate for single-particle excitations and non-Fermiliquid behavior (Metzner, Rohe, and Andergassen, 2003). The dynamical Fermi-surface fluctuations near a Pomeranchuk instability in $d=2$ have been analyzed recently (Dell'Anna and Metzner, 2006): the electronic selfenergy scales as $\omega^{2 / 3}$, thus destroying the Fermi liquid at all wavevectors.

Here we sketch the calculation of the critical behavior - within Fermi-liquid theory - near a spin-symmetric, $d$-wave Pomeranchuk instability, for which the dominant Fermi liquid interaction component is $f_{\mathbf{k k}^{\prime}}=$ $\frac{1}{2 N_{0}} F_{2}^{s} \Sigma_{m} d_{m \hat{\mathbf{k}}}^{*} d_{m \hat{\mathbf{k}}}$, where $d_{m \hat{\mathbf{k}}}$ is one of the $\ell=2$ eigenfunctions. Following Wölfle and Rosch (2007) the corresponding susceptibility is of the form

$$
\begin{aligned}
\chi_{\mathbf{k k}^{\prime}}^{2 m}(\mathbf{q}, \omega) & =d_{m \hat{\mathbf{k}}}^{*} d_{m \hat{\mathbf{k}}^{\prime}} \frac{m}{m^{*}} 2 N_{0} S_{d}(\mathbf{q}, \omega) \\
S_{d}^{-1}(\mathbf{q}, \omega) & =\left(\xi_{0} / \xi\right)^{2}+\xi_{0}^{2} q^{2}-\beta(\mathbf{q}, \omega)-i \gamma(\mathbf{q}, \omega)
\end{aligned}
$$

where

$$
\left(\xi_{0} / \xi\right)^{2}=\frac{m}{m^{*}}\left(1+F_{2}^{s} / 5\right),
$$

in $d=3$ and $\left(\xi_{0} / \xi\right)^{2}=\frac{m}{m^{*}}\left(1+F_{2}^{s}\right)$ in $d=2$ with

$$
\left.\xi_{0}^{2} \approx-\frac{\partial}{\partial q^{2}} \Pi_{d}^{0}(q, 0)\right]_{q=0}, \gamma(\mathbf{q}, \omega)=\frac{m}{m^{*}} \operatorname{Im} \Pi_{d}^{0}(\mathbf{q}, \omega)
$$

and

$$
\Pi_{d}^{0}(\mathbf{q}, \omega)=-\int \frac{d^{d} k}{(2 \pi)^{d}} \frac{f_{\epsilon_{\mathbf{k}+}}^{0}-f_{\epsilon_{\mathbf{k}-}}^{0}}{\omega+i 0-\epsilon_{\mathbf{k}+}+\epsilon_{\mathbf{k}-}}\left|d_{m \hat{\mathbf{k}}}\right|^{2}
$$

where $\epsilon_{\mathbf{k} \pm}=\epsilon_{\mathbf{k} \pm \mathbf{q} / 2}$. Strictly speaking, the formula for $\xi_{0}$ is only a crude estimate as this high-energy property cannot be calculated within Fermi liquid theory (Wölfle and Rosch, 2007), in contrast to the low-frequency damping $\gamma$.

It is important to distinguish two types of modes: "even" modes (with $\ell+m$ even) have $\gamma(q, \omega)=\frac{\omega}{c q}$, where $c$ is the bare Fermi velocity, and $\beta(q, \omega)=0$. For "odd" modes, $\gamma(q, \omega)=\left(\frac{m^{*}}{m}\right)^{2}\left(\frac{\omega}{c q}\right)^{3}$ and $\beta(q, \omega)=2 \frac{m^{*}}{m}\left(\frac{\omega}{c q}\right)^{2}$. Consequently, for even modes we have a dynamical critical exponent $z=3$, whereas for odd modes the 'bare' dynamical critical exponent (ignoring mass renormalizations) is $z=2$. In the Fermi liquid regime, the modes with the highest value of $z$ will dominate. The role of the sub-dominant odd modes and their dynamical critical exponent $z$ depends on the scaling of the prefactor $\frac{m *}{m}$ in $\beta$. We have here a situation of multiple critical exponents, as discussed in Sec. III.H.1.

The correlation length $\xi$ diverges in the limit $F_{2}^{s} \rightarrow-5$. We will be interested in calculating the quasiparticle effective mass and the specific heat as well as the contribution to the electrical resistivity caused by scattering from critical fluctuations. These quantities may be extracted from the quasiparticle self-energy $\Sigma(\mathbf{k}, \omega)$, following Dell'Anna and Metzner (2006), with imaginary part given by (for a lattice system with only one critical mode)

$$
\begin{aligned}
\operatorname{Im} \Sigma(\mathbf{k}, \omega)= & \frac{\left(F_{2}^{s}\right)^{2}}{2 N_{0}}\left|d_{m \hat{\mathbf{k}}}\right|^{2} \int d \omega^{\prime} \int \frac{d^{d} q}{(2 \pi)^{d}}\left[n_{\omega^{\prime}}^{0}+f_{\omega^{\prime}+\omega}^{0}\right] \\
& \times \frac{m}{m^{*}} \operatorname{Im} S_{d}\left(\mathbf{q}, \omega^{\prime}\right) \delta\left(\omega^{\prime}+\omega-\xi_{\mathbf{k}+\mathbf{q}}\right) .
\end{aligned}
$$

In an isotropic system a different averaging has to be used (Wölfle and Rosch, 2007), and for the dominat even modes one can replace $\left|d_{m \hat{\mathbf{k}}}\right|^{2}$ by 1 . After performing the integration over frequency $\omega^{\prime}$ and the component of momentum $q_{r}$, where $\mathbf{q}=q_{r} \hat{\mathbf{k}}_{F}+\mathbf{q}_{t}$, and rescaling $q_{r}=$ $\left(\omega / v_{f}\right) \tilde{q}_{r}, q_{t}=\left(\omega / \xi_{0}^{2} c\right)^{1 / 3} \tilde{q}_{t}$, one finds

$$
\begin{aligned}
\Sigma(\mathbf{k}, \omega)= & i \frac{\left(F_{2}^{s}\right)^{2}}{2 N_{0}} \frac{\Omega_{d-1}}{(2 \pi)^{d}}\left(\frac{\omega}{\xi_{0}^{2} c}\right)^{d / 3} \\
& \times \int d \tilde{q}_{t} \tilde{q}_{t}^{d-1} \ell n\left[1-\frac{i}{\left(\tilde{q}_{t}^{2}+\zeta^{2}\right) \tilde{q}_{t}}\right]
\end{aligned}
$$

where $\zeta=\xi^{-1}\left(\xi_{0}^{2} / \omega\right)^{1 / 3}$. As mentioned above, at the critical point $(\zeta \rightarrow 0) \operatorname{Im} \Sigma(k, \omega) \propto \omega^{d / 3}$.

The contribution to the effective mass from critical fluctuations is obtained from $\frac{m^{*}}{m}=1-$ $\left.\frac{m^{*}}{m} \frac{\partial}{\partial \omega} \operatorname{Re} \Sigma(k, \omega)\right|_{\omega=0}$ as

$$
\frac{m^{*}}{m} \propto \xi^{3-d}
$$

(The factor of $m^{*} / m$ converts a quasiparticle self energy into an electron self energy.) Since the specific-heat coefficient $C / T \propto m^{*} / m$ we have as well $C / T \propto \xi^{3-d}$, in agreement with the result obtained within a bosonic description of the critical dynamics, see Eq. (67): $C / T \propto$ $\left(\xi / \xi_{0}\right)^{z-d}$, considering that $z=3$ in this case.

The contribution to the resistivity is found from $\operatorname{Im} \Sigma$ by the following qualitative argument: Since the typical momentum transfer in electron scattering off a critical fluctuation is $\Delta q \sim \xi^{-1}$, the weight with which such a process contributes to the resistivity is reduced by $\Delta q^{2} \propto$ $1-\cos \theta$, where $\theta$ is the scattering angle. As Eq. (122) gives $\operatorname{Im} \Sigma \propto \xi^{6-d}$, we find for the scattering rate $1 / \tau \propto$ $\xi^{4-d}$, and hence

$$
\Delta \rho \propto \xi^{4-d}\left(T / T_{0}\right)^{2}
$$

Note that a related calculation could be done for a ferromagnetic instability; however, in ferromagnets further complications arise, as described below in Sec. III.H.1. 


\section{H. Breakdown of the Hertz model of a magnetic QCP}

Under what circumstances does the theory of Hertz break down? In this section several possible mechanisms for such a failure of the Landau-Ginzburg-Wilson (LGW) approach in clean systems are highlighted; disorder is briefly discussed in Sec. III.J.

Conceptually, two causes for a failure of the Hertz theory can be identified: (i) A local analytic expansion of the action in terms of the magnetic order parameter does not exist, or (ii) additional degrees of freedom other than magnetism become critical at the transition.

The first situation may arise when, in addition to the order-parameter fluctuations, other (fermionic) slow modes are present in the critical system (as is always the case in metallic magnets). Upon integrating out the fermions, non-analytic non-local or even singular terms may arise, invalidating the approach of Hertz. Then the whole concept, namely to consider an effective description in terms of the critical modes alone, fails - examples to be discussed below are the metallic FM in $d \leq 4$ (Belitz, Kirkpatrick, and Rollbühler, 2004) and the metallic AFM in $d \leq 2$ (Abanov and Chubukov, 2004). A proper critical theory should include both order-parameter and fermionic modes, but such a coupled RG treatment has only been performed in a few cases, see Sec. III.H.1. Note that even in cases when a local expansion of the critical theory in terms of the order parameter is justified, one has make sure that the standard Fermi-liquid form (e.g. the $|\omega|$ term from Landau damping) applies.

The second situation may apply to certain heavyfermion systems and will be discussed in Sec. III.I.

\section{Multiple dynamical exponents: FM QCP}

The metallic ferromagnet is an example where the LGW approach of Hertz fails due to the presence of fermionic modes in the system. The idea can be discussed in terms of time scales: In a nearly-critical quantum system, the length scale $\xi$ may induce several diverging time scales. The order parameter fluctuates on the time scale $\xi_{\tau} \propto \xi^{z_{\mathrm{OP}}}$, with, e.g., $z_{\mathrm{OP}}=3$ in a clean FM according to the theory of Hertz (78). A different time scale is induced by the fermions. In a clean system, electrons cross an ordered domain of size $\xi$ ballistically in the much shorter time $t_{B} \propto \xi^{z_{B}}, z_{B}=1$. In a disordered system, charge or spin (if conserved) diffuse over a distance of $\xi$ in the time $t_{D} \propto \xi^{z_{D}}, z_{D}=2$.

Vojta et al. (1997) [see also Belitz et al. (2000, 2001a); Belitz, Kirkpatrick, and Rollbühler (2004)] have shown that for itinerant quantum critical ferromagnets these other slow modes are indeed important (for AFM the effect is less severe, see Sec. III.H.2.) The problem becomes apparent when deriving the LGW functional from a microscopic theory in perturbation theory. Consider a system with an exchange interaction $H_{J}=$ $-\iint \mathbf{S}(\mathbf{r}) \mathbf{S}\left(\mathbf{r}^{\prime}\right) J\left(\mathbf{r}-\mathbf{r}^{\prime}\right)$, where $\mathbf{S}(\mathbf{r})=\Psi_{\alpha}^{\dagger}(\mathbf{r}) \sigma_{\alpha \alpha^{\prime}} \Psi_{\alpha^{\prime}}(\mathbf{r})$ is the spin density of the electrons, expressed in terms of field operators $\Psi_{\alpha}(\mathbf{r})$ (Kirkpatrick and Belitz, 1996b; Vojta et al., 1997). If $S_{0}[\Psi]$ is the (imaginary-time) action of the Fermi liquid in the absence of the exchange interaction $H_{J}$, then one can rewrite the partition sum formally as a functional integral over the collective field $\phi(\mathbf{r}, \tau)$ with the Hubbard-Stratonovich transformation:

$$
\begin{aligned}
Z & =\int \mathcal{D} \Psi e^{-S_{0}[\Psi]-\sum_{\mathbf{q}} \int_{0}^{\beta} d \tau J_{\mathbf{q}} \mathbf{S}_{\mathbf{q}}(\tau) \mathbf{S}_{-\mathbf{q}}(\tau)} \\
& \propto \int \mathcal{D} \Psi \mathcal{D} \phi e^{-S_{0}[\Psi]-\sum_{\mathbf{q}} \int_{0}^{\beta} d \tau \frac{\phi_{\mathbf{q}}(\tau) \phi_{-\mathbf{q}}(\tau)}{J_{\mathbf{q}}}+\phi_{\mathbf{q}}(\tau) \mathbf{S}_{-\mathbf{q}}(\tau)} \\
& \propto \int \mathcal{D} \phi e^{-\sum_{n=2}^{\infty} S_{n}[\phi]}
\end{aligned}
$$

with

$$
\begin{aligned}
S_{2} & =\frac{1}{\beta V} \sum_{\omega_{n} \mathbf{q}} \phi_{-\mathbf{q},-\omega_{n}}\left(\frac{1}{J_{\mathbf{q}}}-\chi_{\mathbf{q} \omega_{n}}^{(2)}\right) \phi_{\mathbf{q}, \omega_{n}}, \\
S_{n} & =\frac{(-1)^{n+1}}{(\beta V)^{n-1}} \sum \chi^{(n)} \phi_{\mathbf{q}_{1} \omega_{1}} \phi_{\mathbf{q}_{2} \omega_{2}} \ldots \phi_{\mathbf{q}_{\mathbf{n}} \omega_{n}}
\end{aligned}
$$

where $\chi^{(n)}$ are the (connected) $n$-point spin susceptibilities of the reference system $S_{0}$, i.e., the susceptibilities in the absence of the exchange interaction. For a clean Fermi liquid in $d=3$, Belitz, Kirkpatrick, and Vojta (1997) found in perturbation theory in the interactions (of the spin-singlet and Cooper channel)

$$
\begin{aligned}
\chi_{\mathbf{q} \omega_{n}}^{(2)} & \sim c_{1}-c_{2} q^{2}+c_{2}^{\prime} q^{2} \ln \frac{1}{q}-c_{3} \frac{\left|\omega_{n}\right|}{q} \\
\chi^{(4)} & \sim u_{4}-v_{4} \ln \frac{1}{q}
\end{aligned}
$$

or for arbitrary $n, \chi^{(n)} \sim 1 / q^{n-d-1}$. In general, $\chi^{(n)}$ is a complicated non-analytic function of $(n-1)$ momenta and frequencies; we give only the leading singularity for certain limits $q_{n}, \omega_{n} \rightarrow 0$. The presence of these non-analytic corrections in the spin susceptibility of a clean Fermi liquid has later been verified by Chubukov and Maslov (2003, 2004); interestingly, these non-analyticities are absent in the charge channel. Further we note that related non-analytic corrections also show up in the finite-temperature behavior of a Fermi liquid, see Sec. II.D. In a diffusive system in $d=3$, a result similar to (128) holds, with even stronger nonanalyticities (Kirkpatrick and Belitz, 1996b):

$$
\begin{aligned}
\chi_{\mathbf{q} \omega_{n}}^{(2)} & \sim c_{1}-c_{2} q^{2}-c_{2}^{\prime}|q|-c_{3} \frac{\left|\omega_{n}\right|}{q^{2}} \\
\chi^{(4)} & \sim u_{4}+v_{4} \frac{1}{q^{3}}
\end{aligned}
$$

and $\chi^{(n)} \sim 1 / q^{2 n-2-d}$.

The Gaussian action $S_{2}$ close to the QCP is therefore 
given in leading order by

$$
\begin{aligned}
& S_{2}^{c} \approx \frac{1}{\beta V} \sum_{\omega_{n} \mathbf{q}}\left(\delta+c_{2} q^{2}-c_{2}^{\prime} q^{2} \ln \frac{1}{q}+c_{3} \frac{\left|\omega_{n}\right|}{q}\right)\left|\phi_{\mathbf{q}, \omega_{n}}\right|^{2} \\
& S_{2}^{d} \approx \frac{1}{\beta V} \sum_{\omega_{n} \mathbf{q}}\left(\delta+c_{2}^{\prime}|q|+c_{3} \frac{\left|\omega_{n}\right|}{q^{2}}\right)\left|\phi_{\mathbf{q}, \omega_{n}}\right|^{2}
\end{aligned}
$$

for a clean or diffusive metal, respectively.

Does the Gaussian action correctly describe the QCP in leading order? To decide this question we proceed with a scaling analysis. We choose the $c_{2}^{\prime}$ term in $S_{2}^{c}$ and $S_{2}^{d}$ to be dimensionless and therefore $\phi_{\mathbf{q}, \omega} \propto \xi^{\frac{5+z}{2}}$ in the clean and $\phi_{\mathbf{q}, \omega} \propto \xi^{\frac{4+z}{2}}$ in the diffusive metal in $d=3$. The interaction $S_{n}$ in $d=3$ therefore scales proportional to

$$
\begin{aligned}
S_{n}^{c} & \propto\left[\chi^{(n)}\left(\phi_{\mathbf{q}, \omega}\right)^{n}(d \mathbf{q} d \omega)^{n-1}\right] \\
& \propto \xi^{n-4} \xi^{\frac{5+z}{2} n} \xi^{-(n-1)(3+z)} \propto \xi^{-\left(\frac{n}{2}-1\right)(z-1)} \\
S_{n}^{d} & \propto \xi^{2 n-5} \xi^{\frac{4+z}{2} n} \xi^{-(n-1)(3+z)} \propto \xi^{-\left(\frac{n}{2}-1\right)(z-2)}
\end{aligned}
$$

in a clean or diffusive metal, respectively.

What is the value of $z$ ? From the $c_{3}$ term in the Gaussian action (132) one finds that at the QCP the order parameter fluctuates very slowly with $\omega \propto q^{3}$, and therefore $z_{\mathrm{OP}}=3$ both for clean and dirty systems. From this argument, it seems that the contributions from the interactions $(133,134)$ are irrelevant as they vanish for large $\xi$, and therefore it was concluded by Kirkpatrick and Belitz (1996b); Vojta et al. (1997) that the critical theory is described by the Gaussian model $S_{2}$. Afterward, the authors realized (Belitz et al., 2001a) that the simple scaling argument given above is not completely correct. The origin of this failure of "naive" scaling is as we discussed in the beginning of this section - that other slow time scales with dynamical exponent $z_{B}=1$ in the clean metal or $z_{D}=2$ in the diffusive system are induced by the electrons. If we use these values for $z$, we find that all interactions $S_{n}^{d}$ and $S_{n}^{c}$ are marginal, and therefore at least the possibility exists that all of them have to be considered.

As mentioned above, one has to conclude that a description of the QPT in terms of the critical modes alone is not possible for the ferromagnet (Belitz, Kirkpatrick, and Rollbühler, 2004). A generalized critical theory has been set up and analyzed for the ferromagnetic QPT in a diffusive metal (Belitz et al., 2000, 2001a) - this theory involves both the ferromagnetic order-parameter field $\phi$ and the diffusive modes of the disordered metal. Remarkably, the Gaussian critical theory was found to be correct only up to logarithms, as suggested by the scaling with $z=z_{B}=1$ or $z=z_{D}=2$, respectively.

We proceed with a short discussion of the main physical consequences of the non-analytic corrections to the Hertz theory in quantum critical itinerant ferromagnets. In the clean metal, the logarithmic corrections in $S_{2}^{c}$ and $S_{4}^{c}$ lead probably to an instability of the ferromagnetic second-order transition: the $\phi^{4}$ term changes sign at an

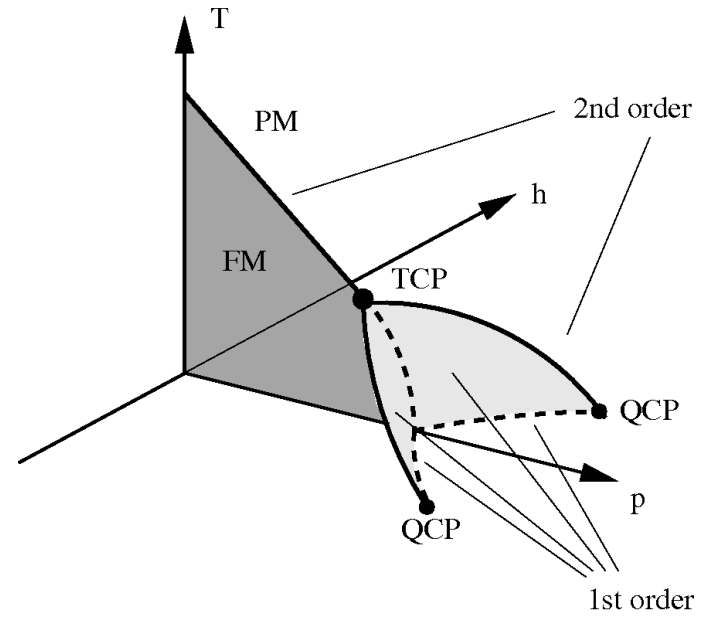

FIG. 4 Generic phase diagram of an itinerant ferromagnet, as function of temperature $T$, tuning parameter $p$, and magnetic field $h$. PM (FM) denote the paramagnetic (ferromagnetic) phases, and TCP is a tricritical point. From Belitz, Kirkpatrick, and Rollbühler, 2005.

exponentially small temperature inducing a weak firstorder transition (Belitz, Kirkpatrick, and Vojta, 1999). Such a first-order transition has, e.g., been reported in $\mathrm{ZrZn}_{2}$, see Sec. IV.B.3. This generic scenario has been discussed by Belitz, Kirkpatrick, and Rollbühler (2005), and the resulting phase diagram is shown in Fig. 4 . The $-q^{2} \ell n 1 / q$ correction in $S_{2}^{c}$ can also drive the transition to an AFM QCP (see Vojta and Sknepnek 2001). At some distance from the first-order or AFM transition, the predictions of the Hertz approach for the 3d ferrmomagnet regarding resistivity and specific heat are likely to hold (up to logarithmic corrections), with a resistivity $\propto T^{5 / 3}$ at the QCP and logarithmically diverging specific-heat coefficient $C / T$. The exponents, e.g., for the pressure dependence of the Néel temperature (94) (Millis, 1993) close to the QCP (but not too close to the first-order transition) require more careful considerations - outside the scope of this review - as they involve directly the scaling dimension of the $\phi^{4}$ term. Chubukov, Pépin, and Rech (2004) have critically studied whether self-energy effects and vertex corrections wash out the singularities that lead to the first-order transition. Interestingly, they found that such corrections can in principle modify critical exponents, but in the case of the ferromagnet the transition remains of first order.

In the disordered diffusive ferromagnet, the Gaussian fixed point is stable, but the exponents deviate strongly from mean-field behavior (Belitz et al., 2000). The quantum critical behavior shows up, e.g., in the resistivity. The $\sqrt{T}$ cusp in the resistivity of a dirty Fermi liquid is modified and a $T^{1 / 3}$ temperature dependence (Belitz et al., 2000) with logarithmic corrections is expected. Similarly the tunneling density of states should display a $\omega^{1 / 3}$ anomaly. More details can be found in Belitz et al. (2000, 2001a). 


\section{Infinitely many marginal operators: AFM QCP in $d=2$}

As $d=2$ is formally the upper critical dimension of the spin-fluctuation theory for the antiferromagnet, one would expect logarithmic corrections to the meanfield behavior as described above. However, Abanov and Chubukov (2004) have shown that in $d=2$ the derivation of the LGW theory breaks down. Somewhat similar to the FM case, the low-energy modes of the Fermi liquid lead to long-range order-parameter interactions. Formally, the coefficients of the high-order interactions in the LGW functional diverge, leading to an infinite number of marginal operators. An analysis of the resulting theory is difficult and again requires a treatment of a coupled field theory of order-parameter fluctuations and fermions - this has not been done until now. Abanov and Chubukov (2004) conclude that the 2d metallic AFM shows a continuous transition with non-trivial exponents, but concrete predictions, e.g., for transport are lacking.

\section{Self-energy effects close to QCP}

The scattering from spin fluctuations strongly modifies the quasiparticles close to the hot lines $\epsilon_{\mathbf{k}_{H}}=\epsilon_{\mathbf{k}_{H} \pm \mathbf{Q}}$ in the vicinity of an AFM QCP. In leading order perturbation theory, the self-energy of those electrons at $T=0$ is given by

$$
\operatorname{Im} \Sigma_{\mathbf{k}}(\Omega) \approx g_{S}^{2} \sum_{\mathbf{k}^{\prime}} \int_{0}^{\Omega} d \omega \operatorname{Im} \chi_{\mathbf{k}-\mathbf{k}^{\prime}}(\omega) \operatorname{Im} g_{\mathbf{k}^{\prime}}^{0}(\omega-\Omega),
$$

where $g_{S}$ is the vertex of the coupling of electrons to spin fluctuations and $g_{\mathbf{k}^{\prime}}^{0}(\omega) \approx 1 /\left(\omega-\epsilon_{\mathbf{k}}+i 0^{+}\right)$is the Green function of the (free) fermions. Using $1 / \chi_{\mathbf{q} \pm \mathbf{Q}}(\omega) \sim \mathbf{q}^{2}+$ $(i \omega)^{2 / z_{\mathrm{OP}}}$ and $(135)$ we obtain at the AFM QCP

$$
\operatorname{Im} \Sigma_{\mathbf{k}_{H}+\delta \mathbf{k}}(\Omega) \sim \Omega^{1+\frac{d-3}{z \mathrm{OP}}} f\left(\frac{(\delta \kappa)^{2}}{\Omega^{2 / z_{\mathrm{OP}}}}\right)
$$

where $\delta \kappa \sim \delta \mathbf{k} \cdot \mathbf{v}_{\mathbf{k}_{H}+\mathbf{Q}}$ is a measure for the distance from the hot line, and $f$ is some scaling function with $f(x \rightarrow 0) \sim$ const and $f(x \rightarrow \infty) \sim 1 / x^{\frac{5-d}{2}}$.

Obviously a Fermi-liquid description of the electrons, which requires $\operatorname{Im} \Sigma_{\mathbf{k}}\left(\epsilon_{\mathbf{k}}\right)<\epsilon_{\mathbf{k}}$ for $k \rightarrow k_{F}$, breaks down for $d<3$ for quasiparticles with momentum $\mathbf{k}_{H}$. In this sense, the critical dimension for a breakdown of FL theory is $d=3$, even within the Hertz approach (which formally has $d_{c}^{+}=2$ ). For quantum critical antiferromagnets this breakdown of Fermi liquid for $d<3$ affects, however, only a tiny fraction of the quasiparticles. At present it is not clear whether this effect will influence the spin dynamics. The RG analysis of the theory of Hertz (77) suggests that this is not the case, however, this question should be addressed in a RG treatment which includes both fermionic and bosonic modes.

Let us contrast this scenario with the $Q=0$ situation of a metallic ferromagnet or a Pomeranchuk instability.
At the QCP the susceptibility is of the form $1 / \chi_{\mathbf{q}}(\omega) \sim$ $q^{\alpha}+(i \omega) / q^{z_{\mathrm{OP}}-\alpha}$ with $\alpha=2$ and $z_{\mathrm{OP}}=3$ (supplemented by logarithmic corrections in $3 \mathrm{~d}$ ). Then, the self-energy is momentum independent,

$$
\operatorname{Im} \Sigma_{\mathbf{k}}(\Omega) \sim \Omega^{1+\frac{d-(1+\alpha)}{z_{\mathrm{OP}}}},
$$

leading to a marginal behavior in $d=3$, and a breakdown of the Fermi liquid in $d=2$ due to the $\omega^{2 / 3}$ dependence of the self-energy, here over the entire Fermi surface due to $Q=0$ (see also Sec. III.G). Then, it is natural to suspect that the precondition for the Hertz theory are no longer fulfilled. Chubukov, Pépin, and Rech (2004) have investigated in detail the role of self-energy effects for ferromagnetic quantum transitions. Indeed, those effects are found to be relevant and would modify critical exponents, if the transition were not of first order as concluded by the authors, see Sec. III.H.1.

\section{Pseudogaps close to $\mathrm{QCP}$}

In the antiferromagnetically ordered phase of a metal, gaps open in parts of the Fermi surface with $\epsilon_{\mathbf{k}} \approx \epsilon_{\mathbf{k} \pm \mathbf{Q}} \approx$ 0 , provided that the Fermi volume is sufficiently large. (As already mentioned in Sec. III.C.1, this feature is not captured by the LGW order-parameter theory of Hertz - this theory is not valid in the ordered phase, e.g., it wrongly predicts damped Goldstone modes.) It is therefore important to ask whether precursors of these gaps will show up already in the paramagnetic phase close to the quantum critical point. In this regime, the behavior of the system is dominated by large antiferromagnetic domains of size $\xi$, slowly fluctuating on the time scale $\tau_{\xi} \sim \xi^{z_{\mathrm{OP}}}$ where $z_{\mathrm{OP}}$ is the dynamical critical exponent of the order parameter. As $\xi$ is diverging when the QCP is approached, it is suggestive (Schrieffer, 1995) to assume that the electrons will adjust their wave functions adiabatically to the local antiferromagnetic background and will therefore show a similar behavior as in the AFM ordered phase. Will precursors of this effect show up and induce pseudogaps in the paramagnetic phase for sufficiently large $\xi$ ? This would imply a breakdown of the LGW approach of Hertz. Below we will try to estimate this effect using a simple qualitative scaling analysis, for details see Rosch (2001). Pseudogaps play an important role in the physics of underdoped cuprates (Anderson, 1997) and it has been speculated that they are indeed precursors of gaps in either superconducting, antiferromagnetic, flux or striped phases (not discussed in this review).

To define the concept of a pseudogap more precisely, we first analyze the ordered phase where a proper meanfield Hamiltonian of the electrons is of the form

$$
H_{\Delta}=\sum_{\sigma, \mathbf{k}}\left(c_{\sigma, \mathbf{k}}^{\dagger}, c_{\sigma, \mathbf{k}+\mathbf{Q}}^{\dagger}\right)\left(\begin{array}{cc}
\epsilon_{\mathbf{k}} & \sigma \Delta \\
\sigma \Delta & \epsilon_{\mathbf{k}+\mathbf{Q}}
\end{array}\right)\left(\begin{array}{c}
c_{\sigma, \mathbf{k}} \\
c_{\sigma, \mathbf{k}+\mathbf{Q}}
\end{array}\right) .
$$


$\Delta$ is proportional to the staggered order parameter (assumed to point in $z$ direction) and the $\mathbf{k}$ sum extends over a magnetic Brillouin zone. Close to the "hot points" ("hot lines" in three dimensions) with $\epsilon_{\mathbf{k}_{H}}=\epsilon_{\mathbf{k}_{H} \pm \mathbf{Q}}=0$ a gap opens and the band structure at $\mathbf{k}=\mathbf{k}_{H}+\delta \mathbf{k}$ is approximately given by

$$
\epsilon_{\delta \mathbf{k}}^{ \pm} \approx \frac{\mathbf{v}_{1}+\mathbf{v}_{2}}{2} \delta \mathbf{k} \pm \frac{1}{2} \sqrt{\left[\left(\mathbf{v}_{1}-\mathbf{v}_{2}\right) \delta \mathbf{k}\right]^{2}+4 \Delta^{2}}
$$

where $\mathbf{v}_{1}=\mathbf{v}_{\mathbf{k}_{H}}$ and $\mathbf{v}_{2}=\mathbf{v}_{\mathbf{k}_{H}+\mathbf{Q}}$ are the Fermi velocities close to the hot points. Interactions will actually induce some small weight within these gaps but this does not invalidate the mean-field picture.

Within the LGW theory (77) of the AFM QCP (Hertz, 1976; Millis, 1993) no precursor of the gap shows up, since spin-spin interactions are irrelevant by power counting. In the discussion of the ferromagnetic QPT in Sec. III.H.1, we have seen that these arguments are not reliable in the case of a quantum phase transition in a metal, as generally a second dynamical critical exponent $z_{F}$ exists which describes that, e.g., ballistic fermions can traverse a domain of size $\xi$ in a time $\tau_{F} \propto \xi^{z_{F}}$ with $z_{F}=1$.

For the following argument, we assume that the susceptibility at the QCP has the form $1 / \chi_{\mathbf{q} \pm \mathbf{Q}}(\omega) \sim q^{2}+$ $(i \omega)^{2 / z_{\mathrm{OP}}}$. We are mainly interested in the case $z_{\mathrm{OP}}=2$, smaller values for $z_{\mathrm{OP}}$ might be relevant if pseudogap formation takes places, larger values have, e.g., been used to fit experiments (Schröder et al., 1998) in $\mathrm{CeCu}_{6-x} \mathrm{Au}_{x}$. For our argument, we assume that the quasiparticles move in a (quasi-static) staggered field with the effective size $\Delta$ (to be determined later). According to the mean-field result (139) a gap of size $\omega^{*}=\Delta$ opens in a $(d-2)$ dimensional stripe in momentum space of width $k^{*}=\Delta / v_{F}$. Interactions can change this (see (136)) to $\omega^{*} \sim\left(k^{*}\right)^{z_{F}} \sim \Delta^{z_{F}}$ where $z_{F}=1$ is the mean-field exponent. Heisenberg's uncertainty relation dictates that the electrons have to see a quasi-static AFM background for a time $\tau^{*} \gtrsim 1 / \omega^{*}$ on a length scale of order $\xi^{*} \gtrsim 1 / k^{*}$ perpendicular to the direction of the hot lines to develop the pseudo gap. What is the effective size of the quasi-static AFM order $\langle\phi\rangle_{\xi^{*}, \tau^{*}}^{\text {eff }}$ on these length and time scales? The following estimate should at least give an upper limit at the QCP:

$$
\begin{aligned}
\left(\langle\phi\rangle_{\xi^{*}, \tau^{*}}^{\mathrm{eff}}\right)^{2} & \lesssim \int_{0}^{\omega^{*}} d \omega \int_{q_{\perp}<k^{*}} d^{2} q_{\perp} \int_{-\infty}^{\infty} d^{d-2} q_{\|} \operatorname{Im} \chi_{\mathbf{q} \pm \mathbf{Q}} \\
& \sim\left(k^{*}\right)^{d+z_{\mathrm{OP}}-2}+\left(k^{*}\right)^{2}\left(\omega^{*}\right)^{\frac{d+z_{\mathrm{OP}}-4}{z_{\mathrm{OP}}}} \\
& \sim \Delta^{\left(d+z_{\mathrm{OP}}-4\right) \frac{z_{F}}{z_{\mathrm{OP}}}+2}
\end{aligned}
$$

where the anisotropic integration of $q$ takes into account that the momentum of the electrons parallel to the hot line can vary on the scale $k_{F}$. In (141) we assumed $z_{F} \leq$ $z_{\mathrm{OP}}$.

If we assume furthermore that $\Delta$ is proportional to $\langle\phi\rangle_{\xi^{*}, \tau^{*}}^{\text {eff }}$ as suggested by the mean field analysis (which should be valid above the upper critical dimension), we obtain the inequality $\Delta^{2} \lesssim$ const $\cdot \Delta^{\left(d+z_{\mathrm{OP}}-4\right) \frac{z_{F}}{z_{\mathrm{OP}}}+2}$. This implies that, at least in a weak-coupling situation, pseudogaps can appear only for

$$
d+z_{\mathrm{OP}} \leq 4
$$

Note that it is accidental that Eq. (142) coincides with the condition for the relevance of the $\phi^{4}$ interaction (79) in the Hertz model as is evident from the fact that $z_{F}$ enters Eq. (141). Within the approach of Hertz, $z_{\mathrm{OP}}=$ 2 and the critical dimension for pseudogap formation is therefore $d_{c}=2$, i.e., no pseudogaps are expected in $d=3$ as long as interactions are not too strong.

The derivation of Eq. (142) is based on a number of assumptions. The estimate (140) of $\langle\phi\rangle^{\text {eff }}$ and therefore (142) is based on the existence of amplitude fluctuations of the staggered order parameter which destroy the pseudogap. Electrons can adjust their wave functions much better adiabatically to angular fluctuations of the direction of the staggered magnetization than to fluctuations of its size. Schrieffer (1995) has argued that pseudogap behavior will occur always sufficiently close to an AFM QCP. For his argument, he considered models without amplitude fluctuations. Within the theory of Hertz, however, amplitude fluctuations are present close to the AFM QCP in $d=3$ because the system is above its upper critical dimension. Furthermore, strong statistical interactions of the electrons with the magnetic excitations (see Rosch (2001) and references therein) might destroy pseudogaps even in the absence of amplitude fluctuations.

\section{Itinerant AFM with $Q=2 k_{F}$}

In our previous discussions of QCP of nearly AFM metals we have assumed a large Fermi volume with $Q<2 k_{F}$, where the spin fluctuations couple directly to quasiparticles with $\epsilon_{\mathbf{k}_{H}} \approx \epsilon_{\mathbf{k}_{H}+\mathbf{Q}} \approx \epsilon_{F}$ along "hot" lines on the Fermi surface $(d=3)$. In the opposite case, $Q>2 k_{F}$, the spin fluctuations decouple in leading order from the quasiparticles due to energy and momentum conservation and their dynamics follows from the conventional $\omega^{2}$ term as in magnetic insulators, Eq. (63).

A special case is $Q=2 k_{F}$. In $d=3$ the hot lines shrink to a single point with parallel Fermi velocities $v_{\mathbf{k}_{H}} \| v_{\mathbf{k}_{H}+\mathbf{Q}}$ for $\epsilon_{\mathbf{k}_{H}}=\epsilon_{\mathbf{k}_{H}+\mathbf{Q}}=\epsilon_{F}$. In this situation, resonant scattering of the spin fluctuations from the electrons leads to a complete breakdown (Millis, 1993) of the LGW expansion (77) underlying the Hertz theory of a QCP. This can be seen from a direct calculation of the connected $n$-point function $\chi^{n}(127)$ in the limit where all momenta are set to $\mathbf{Q}$ and all frequencies to 0 . In this limit, the effective $n$-paramagnon interaction diverges for 
even $n$ in the low- $T$ limit

$$
\begin{aligned}
\chi^{n} & \sim \frac{1}{\beta V} \sum_{\omega_{n}, \mathbf{k}}\left(\frac{1}{i \omega_{n}-\epsilon_{\mathbf{k}}}\right)^{n / 2}\left(\frac{1}{i \omega_{n}-\epsilon_{\mathbf{k}+\mathbf{Q}}}\right)^{n / 2} \\
& \sim \begin{cases}1 / T^{n-4} & \text { for } d=3 \\
1 / T^{n-5 / 2} & \text { for } d=2\end{cases}
\end{aligned}
$$

Our result in $d=3$ differs from the formula of Millis (1993); we find that the contribution $\sim 1 / T^{n-3}$ vanishes exactly. A simple scaling analysis with $k \sim 1 / L$, $\omega \sim T \sim 1 / L^{2}, \phi(\mathbf{r}, \tau) \sim L^{1-(d+2) / 2}$ shows that $S_{n}$ in (127) diverges with $S_{n} \sim L^{n / 2-3}$ in $d=3$ and $S_{n} \sim L^{n-1}$ in $d=2$. Interactions of arbitrarily high $n$ are therefore relevant and the LGW expansion in terms of the order parameter (77) breaks down completely. A critical theory cannot be formulated in terms of order-parameter fluctuations alone.

However, a weak-coupling analysis suggests that $Q=$ $2 k_{F}$ is not realized in generic three-dimensional systems. The reason is that in (126) the polarizability of noninteracting fermions

$$
\chi_{\mathbf{q}}^{(2)}(\omega=0)=\sum_{\mathbf{k}} \frac{f\left(\epsilon_{\mathbf{k}}\right)-f\left(\epsilon_{\mathbf{k}+\mathbf{q}}\right)}{\epsilon_{\mathbf{k}}-\epsilon_{\mathbf{k}+\mathbf{q}}},
$$

where $f(\omega)$ is the Fermi function, does not peak at $q=2 k_{F}$. This is not only true for a quadratic dispersion, but also for any band structure in the absence of perfect nesting. Whether interaction effects can stabilize an AFM QCP with $Q=2 k_{F}$ in the absence of perfect nesting is not known.

In $d=2$, however, a spin-density wave transition with $Q=2 k_{F}$ is very likely, as the polarizability of noninteracting electrons $\chi_{\mathbf{q}}^{(2)}$ is typically peaked at $2 k_{F}$. Altshuler, Ioffe, and Millis (1995) analyzed such a situation and concluded that the strong interactions will probably induce a first-order transition. In this sense, the QCP is destroyed.

\section{Superconductivity}

Generically, the spin fluctuations induce an attractive interaction between the quasiparticles (Abanov, Chubukov, and Finkelstein, 2001; Monthoux and Lonzarich, 1999). Accordingly, one can expect a superconducting phase close to a magnetic QCP of a metal as it is observed in sufficiently clean samples, see Sec. IV.C. Superconductivity is outside the scope of this review; here we just note that the order parameter is probably unconventional and that the superconducting phase will change the dynamical critical exponent $z_{\mathrm{OP}}$ and therefore the critical behavior of the antiferromagnetic QCP due to a suppression of the spin-wave damping in the presence of gaps. In this sense the LGW theory (77) of Hertz breaks down due to superconductivity. In $d=3$, the superconducting phase appears typically at very low temperature (Sec. IV.C), and the Hertz theory remains valid at temperatures above the superconducting $T_{c}$. The situation may be different in (quasi-)2d systems, where Cooper pairs form at much higher temperatures (Abanov, Chubukov, and Finkelstein, 2001; Monthoux and Lonzarich, 1999), effectively reducing the damping of spin fluctuations.

\section{Breakdown of the Kondo effect in heavy-fermion metals}

For heavy-fermion systems (HFS) it is generally accepted that the magnetic transition is driven by a competition of the lattice Kondo effect, which favors a paramagnetic ground state, and a magnetic RKKY or superexchange interaction between the local $f$ moments (Doniach, 1977). In the heavy Fermi-liquid state the local moments contribute to the Fermi volume leading to a "large" Fermi volume, see Sec. II.F.2 - the $f$ electrons are usually termed "delocalized" in this situation.

Before discussing scenarios for the magnetic transition we have to think about the nature of the ordered phase in HFS (the state on the l.h.s. of Fig. 1). Two distinct types of magnetically ordered metals appear possible. (i) The magnetism can arise from a spin-density wave instability of the parent heavy FL state. Here, Kondo screening is essentially intact, with a weak polarization of the local moments which are still "delocalized". We will refer to such a state as the SDW metal. (ii) A different kind of magnetic metal is possible where the localized moments order due to RKKY exchange interactions, and do not participate in the Fermi volume, i.e., Kondo screening is absent. We will denote this second state, which can be expected to be realized deep in the ordered phase (Yamamoto and Si, 2007), as a "local-moment magnetic (LMM) metal". The distinction between these two kinds of states can be drawn sharply, if the Fermi surfaces have different topologies (albeit the same volume modulo that of the Brillouin zone of the ordered state), such that they cannot be smoothly connected to one another.

Returning to the transition from the paramagnet to the antiferromagnet, one possibility is that the Fermi liquid undergoes a transition to a SDW metal - this QCP is well described by the LGW approach of Hertz, Eq. (77). In this situation the local moments remain screened across the phase transition, i.e., a suitably defined lattice Kondo temperature stays finite at the QCP. The anomalous behavior close to AFM QCP in heavy-fermion systems like $\mathrm{CeCu}_{6-x} \mathrm{Au}_{x}$ and $\mathrm{YbRh}_{2} \mathrm{Si}_{2}$ (discussed in detail in Sec. IV.A) - inconsistent with the Hertz scenario - has stimulated discussions about a different transition (Coleman, 1999; Schröder et al., 1998, 2000; Senthil, Sachdev, and Vojta, 2003; Senthil, Vojta, and Sachdev, 2004; Si, Smith, and Ingersent, 1999; Si et al., 2001, 2003): If the ordered state is a LMM metal, the transition to be considered now involves the breakdown of Kondo screening (due to competing magnetic fluctuations), accompanied by an abrupt change of the Fermi surface. This is an 
exciting scenario, as the complete collapse of the Fermi surface is in a sense the most drastic violation of the assumptions of the Hertz theory. No local order parameter can be defined, and the LGW approach fails. Instead, the criticality is carried by emergent degrees of freedom associated with the Kondo effect.

Our present theoretical understanding of such transitions is limited, and we will describe a few theoretical approaches below. An obvious question then is: Can there be a continuous transition where the Kondo screening disappears concomitantly with the appearance of magnetic long-range order? This will be discussed in Sec. III.I.4. Parenthetically, we note that some materials show a first-order volume collapse transition at finite $T$ (McMahan et al., 1998) - in contrast, we are interested here in a continuous transition at $T=0$.

A zero-temperature transition involving the breakdown of Kondo screening ("Kondo transition") implies a collapse of the Fermi surface - in fact, we use this as a defining criterion for a Kondo transition. Experimentally, the collapse of the Fermi surface may be detected via photoemission or de Haas-van Alphen measurements, and transport properties like the Hall conductivity will show a jump upon crossing the transition at lowest $T$ (Coleman et al., 2001; Coleman, Marston, and Schofield, 2005; $\mathrm{Si}$, Smith, and Ingersent, 1999). (At a SDW transition the Fermi surface evolves continuously, and the Hall coefficient displays a kink, but no jump; only at a magneticfield driven transition does the derivative of the Hall current with respect to the magnetic field jump.) As the Kondo transition is not associated with a single critical (fermionic) wavevector, one may expect critical fluctuations in an extended range of the reciprocal space. These qualitative theoretical considerations fit remarkably well some recent experiments: In $\mathrm{CeCu}_{6-x} \mathrm{Au}_{x}$ (Sec. IV.A.1) the susceptibility at the AFM QCP was found to obey $1 / \chi(\mathbf{q}, \omega) \sim f(\mathbf{q})+(-i \omega+a T)^{\alpha}$ (Eq. 149 below) with an anomalous exponent $\alpha \sim 0.8$, obtained from fits to susceptibility measurements and inelastic neutron scattering data at various positions in momentum space including $\mathbf{q}=0$ (Schröder et al., 1998, 2000). Momentum and frequency dependence "separate", this favors an interpretation in terms of a Kondo transition. For $\mathrm{YbRh}_{2} \mathrm{Si}_{2}$ (Sec. IV.A.5) no neutron scattering data are available to date. However, a recent magneto-transport measurement may indicate a jump in the Hall coefficient at the magnetic QCP (Paschen et al., 2004); in addition, magneto-striction data of Gegenwart et al. (2007) show the vanishing of several energy scales at the same QCP of $\mathrm{YbRh}_{2} \mathrm{Si}_{2}$.

Let us emphasize that the breakdown of Kondo screening does not imply that the local moments are free to fluctuate at the QCP: The critical behavior will be manifested in anomalous power laws in the spin correlations, as shown explicitly, e.g., within the scenario of "local criticality" described below. Similarly, there will be no $\ell n 2$ entropy per spin at the QCP or in the quantum critical region. Thus the characteristic temperature $T_{1 / 2}$, where the magnetic entropy equals $0.5 \ln 2$, is not expected to go to zero at the QCP - this is in fact consistent with specific heat data on both $\mathrm{CeCu}_{6-x} \mathrm{Au}_{x}$ (Fig. 8 below) and $\mathrm{YbRh}_{2} \mathrm{Si}_{2}$.

In a simple scenario, where the low-temperature state of the conduction electrons with small Fermi volume is adiabatically connected to high temperatures, a characteristic signature of a breakdown of Kondo screening may be a shift of the maximum temperature in the resistivity, $T_{\mathrm{m}}$, to lower temperatures upon approaching the QCP (as this may signal the crossover from "small" to "large" Fermi volume). However, concrete calculations for the transport crossover are lacking, and moreover this picture seems not to be supported experimentally: In $\mathrm{CeCu}_{6-x} \mathrm{Au}_{x}, T_{\mathrm{m}}$ (for $\rho$ measured along the $a$ direction) decreases smoothly across $x_{c}$ and vanishes at $x \approx 0.16$ (v. Löhneysen et al., 2002).

We note that arguments have been put forward (Maebashi, Miyake, and Varma, 2005) for a generic breakdown of the single-impurity Kondo effect at an antiferromagnetic QCP; the consequences for lattice models have not been studied in detail.

\section{1. "Local" QCP within extended DMFT}

A first approach designed to capture the breakdown of the lattice Kondo effect due to magnetic bulk fluctuations employs an extension of the dynamical mean-field theory (DMFT) and has been worked out by $\mathrm{Si}$, Smith, and Ingersent (1999); Si et al. (2001, 2003); Smith and Si (2000). It led to the proposal of a "local" QCP (to be made precise below), based on the idea that the breakdown of Kondo screening is a spatially local phenomenon, i.e., it affects every spin of the underlying Kondo lattice independently.

The starting point is the Kondo lattice model, where localized spins $\mathbf{S}_{i}$ couple to the spin density of conduction electrons at lattice site $i, \mathbf{s}_{i}=c_{i \alpha}^{\dagger} \sigma_{\alpha \beta} c_{i \beta} / 2$, with

$$
H=\sum_{k \sigma} \epsilon_{k} c_{k \sigma}^{\dagger} c_{k \sigma}+J \sum_{i} \mathbf{S}_{i} \cdot \mathbf{s}_{i}+\sum_{i, j} I_{i, j} \mathbf{S}_{i} \cdot \mathbf{S}_{j}
$$

where a direct spin-spin exchange term $(I)$ has been added to the usual Kondo lattice model, see Sec. II.F.2. While the usual DMFT maps the lattice problem to a single impurity in a fermionic bath, the extended DMFT (EDMFT) uses a mapping to a so-called Bose-Fermi Kondo model with both a fermionic and bosonic baths (represented by operators $c_{k}$ and $b_{k}$ ):

$$
\begin{aligned}
H_{\mathrm{loc}}= & \sum_{k \sigma} E_{k} c_{k \sigma}^{\dagger} c_{k \sigma}+J \mathbf{S} \cdot \mathbf{s}_{0}+ \\
& +\gamma_{0} \sum_{k} \mathbf{S} \cdot\left(\mathbf{b}_{k}+\mathbf{b}_{-k}^{\dagger}\right)+\sum_{k} \omega_{k} \mathbf{b}_{k}^{\dagger} \mathbf{b}_{k},(145)
\end{aligned}
$$

see also Eq. (56) in Sec. II.F.4. Within EDMFT, the Green's functions and susceptibilities of the lattice model 
are approximated by $1 / g_{\mathbf{k}}(\omega) \approx \omega-\epsilon_{\mathbf{k}}-\Sigma(\omega)$ and $1 / \chi_{\mathbf{q}}(\omega) \approx I_{\mathbf{q}}+M(\omega)$, where $\Sigma(\omega)$ and $M(\omega)$ are the electron and $b$ self-energies of the local problem. The free parameters $E_{k}, \omega_{k}$ and $g$ are determined from the self-consistency condition that the local Green's function and susceptibility in the global and local model, (144) and (145), match. Formally, EDMFT can be justified within a certain $d \rightarrow \infty$ limit (Smith and Si, 2000) but it may be used as an approximation to a finite-dimensional system as long as the physics is not dominated by longrange spatial fluctuations.

Within EDMFT, it is possible to describe situations where collective magnetic fluctuations destroy the Kondo effect. The Bose-Fermi Kondo model (145) is known to have a continuous QPT, due to the competition of the two baths, between a phase with Kondo screening and one with universal local-moment fluctuations - see Sec. II.F.4. The QCP of the lattice model (144) is thus mapped - via EDMFT - onto the impurity QCP of Eq. (145), where the magnetic instability of the lattice drives the Kondo effect critical. At this "local" QCP all self-energies are momentum-independent, and the nonlocal dynamics of the magnetic fluctuations is Gaussian.

A qualitative analysis of the EDMFT equations in $d=$ 2 (Si et al., 2003) shows that the logarithmic divergence of the local susceptibility at the QCP can cause a power-law behavior of $M(\omega)$ at $T=0, M(\omega)=-I_{\mathbf{Q}}+(-i \omega / \Lambda)^{\alpha}$, where $\mathbf{Q}$ is the ordering vector, $\Lambda$ a cutoff, and $\alpha$ a non-universal exponent. A numerical solution of a simplified EDMFT (without fermionic self-consistency and with Ising magnetic symmetry) has confirmed this result (Grempel and Si, 2003; Zhu, Grempel, and Si, 2003). The impurity critical point has been shown to feature $\omega / T$ scaling in $\chi$. These results are in remarkable agreement with what has been found in the experiments of Schröder et al. (2000), see Eq. (149) below - in particular the anomalous exponent of the susceptibility is obtained as $\alpha \approx 0.72$ (Grempel and Si, 2003) while the value from fitting the experimental data is $\alpha \approx 0.74$. Let us point out that, in this theory, the occurrence of $\omega / T$ scaling, despite the non-local magnetic dynamics being Gaussian, is caused by the non-LGW character of the critical point, where the leading singularities are driven by local physics controlled by an interacting impurity QCP.

As explicitly discussed by Si, Smith, and Ingersent (1999); Si et al. (2003), the collapse of the Kondo scale necessarily leads to a jump in the Fermi volume upon crossing the transition at $T=0$. The destruction of the Fermi surface is expected to cause non-Fermi liquid behavior in the resistivity at the QCP, however, concrete theoretical predictions for transport at finite temperatures are lacking to date.

One issue in the DMFT description of bulk criticality is related to the zero-point entropy: Impurity critical points generically display a finite residual entropy (Vojta, 2006a); this implies an extensive entropy for the bulk system (which would render the corresponding fixed point extremely unstable). In the case of the Bose-Fermi
Kondo model (145) a reliable calculation of the impurity entropy is not available to date, however, it is conceivable that it vanishes in the limit $d \rightarrow 2^{+}$, circumventing this problem.

\section{Fractionalized Fermi liquid and deconfined criticality}

A different approach to the breakdown of Kondo screening, without any assumptions about spatial locality, starts by identifying the zero-temperature phase which arises when Kondo screening breaks down without the simultaneous onset of magnetic order (or other types of symmetry breaking). As has been detailed by Senthil, Sachdev, and Vojta (2003), the resulting state is a paramagnet where the conduction electrons form well-defined quasiparticles on their own and the local moments are in a fractionalized spin-liquid state - this phase represents a metallic spin-liquid state and has been termed "fractionalized Fermi liquid" (FL*). The spin-liquid component may be gapped or gapless, and may feature a secondary instability to an ordered state, see Sec. III.I.4.

Technically, the transition from $\mathrm{FL}^{*}$ to $\mathrm{FL}$, at $T=0$ as a function of some control parameter like pressure, can be analyzed in slave-boson mean-field theory plus Gaussian fluctuations around the saddle point (Senthil, Vojta, and Sachdev, 2004). The mean-field Hamiltonian for the Kondo-lattice model (144) reads

$$
\begin{aligned}
H_{\mathrm{mf}} & =\sum_{k} \epsilon_{k} c_{k \alpha}^{\dagger} c_{k \alpha}-\chi_{0} \sum_{\left\langle r r^{\prime}\right\rangle}\left(f_{r \alpha}^{\dagger} f_{r^{\prime} \alpha}+\text { h.c. }\right) \\
& +\mu_{f} \sum_{r} f_{r \alpha}^{\dagger} f_{r \alpha}-b_{0} \sum_{k}\left(c_{k \alpha}^{\dagger} f_{k \alpha}+\text { h.c. }\right)
\end{aligned}
$$

where $\mathbf{S}_{r}=\frac{1}{2} f_{r \alpha}^{\dagger} \sigma_{\alpha \alpha^{\prime}} f_{r \alpha^{\prime}}$ is the auxiliary-fermion representation of the local moments, and $\chi_{0}, \mu_{f}$, and $b_{0}$ are mean-field parameters.

The QPT from $\mathrm{FL}^{*}$ to FL involves a change from a small Fermi volume, containing only conduction electrons, to a large Fermi volume including all Kondo spins - on the mean-field level this is signaled by the condensation of the slave boson $b_{0}$ measuring the hybridization between the $c$ and $f$ bands. (Beyond mean field, a compact gauge field needs to be introduced to implement the local constraint of the $f$ fermions; this, e.g., suppresses a finite-temperature phase transition.) It is illuminating to discuss the Fermi surface properties: Close to the transition the Fermi surface consists of two sheets with primarily $c$ and $f$ character, respectively. Approaching the QPT from the FL side, the quasiparticle weight on an entire sheet of the Fermi surface vanishes continuously - this illustrates how a discontinuous jump in the Fermi volume can happen at a continuous transition. Clearly, the transition is not associated with a specific critical (fermionic) wavevector, but it is also not spatially local, as all self-energies retain their momentum dependence.

The critical theory of the FL-FL* transition can be derived starting from the slave-particle formulation, 


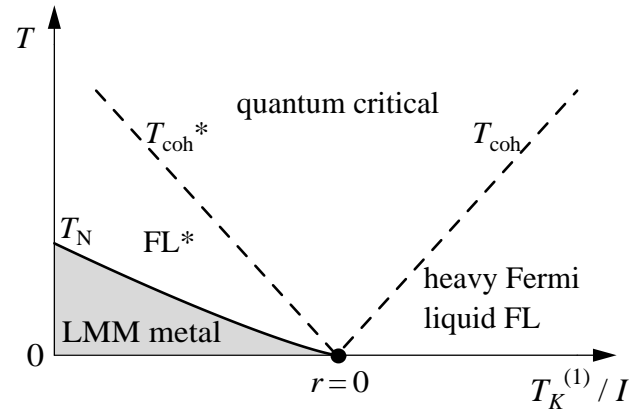

FIG. 5 Phase diagram near the conjectured FL-LMM quantum transition of a heavy-fermion metal (see also Fig. 1). The primary phase transition, characterized by the breakdown of Kondo screening, is between the heavy Fermi liquid (FL) and fractionalized Fermi liquid $\left(\mathrm{FL}^{*}\right)$. $\mathrm{FL}^{*}$ is unstable at low energies towards local-moment magnetism. Two distinct energy scales are present on the l.h.s., manifested by the differing exponents by which $T_{\mathrm{N}}$ and $T_{\mathrm{coh}}$ (or $T_{\mathrm{coh}}^{*}$ ) approach the quantum critical point. From Senthil, Sachdev, and Vojta, 2005.

Eq. (146), supplemented by a gauge field. Provided that the two Fermi surfaces do not overlap, the fermions can be integrated out, and one ends up with a theory for dilute bosons $b$ coupled to a compact $\mathrm{U}(1)$ gauge field. The transition is tuned by the chemical potential of the bosons; it occurs at the (bosonic) wavevector $Q=0$ and has dynamical exponent $z=2$, it is thus above its upper critical dimension. The FL coherence temperature vanishes as the transition is approached from the FL side as $T_{\text {coh }} \propto|r|$. The specific heat acquires a singular contribution from gauge-field fluctuations with $C / T \sim \ell n(1 / T)$ in $d=3$, resembling the experimental result on $\mathrm{CeCu}_{6-x} \mathrm{Au}_{x}$. A preliminary transport calculation (Senthil, Vojta, and Sachdev, 2004), taking into account the scattering of the critical bosons off gauge-field fluctuations, led to a resistivity $\rho \sim 1 / \ell n(1 / T)$, inconsistent with experiments. Interestingly, the decay of the bosons into particle-hole pairs becomes possible above an energy $E^{*}$ which can be small if the distance between the two Fermi surfaces is small; above this energy the theory obeys $z=3$, and an additional $\ell n(1 / T)$ contribution in $C / T$ appears (Paul et al., 2007). In this regime, the resistivity has been estimated as $\rho \sim T \ell n(T)$. Coleman, Marston, and Schofield (2005) have calculated the $T=0$ Hall coefficient using the model (146), and found a jump when passing through the QPT. Clearly, more detailed transport studies, also taking into account impurity scattering, are required.

So far, magnetism is not involved in this scenario. Clearly, the spin liquid in the $\mathrm{FL}^{*}$ phase is potentially unstable towards magnetic order at low $T$ - the resulting state will be a LMM metal. A particularly appealing scenario is that this instability arises as a secondary one, driven by an operator that is irrelevant at the QCP (Fig. 5). This naturally leads to a Landau-forbidden transition within the concept of deconfined criticality, discussed below in Sec. III.I.4. We caution, however, that explicit calculations using slave-particle theories, taking into account magnetism, result in two separate critical points for magnetism and the Kondo effect, at variance with Fig. 5 (Senthil, Vojta, and Sachdev, 2004).

\section{Spin-charge separation at the QCP}

A related scenario for the breakdown of Kondo screening has been proposed by Pépin (2005). It is based on the idea that the heavy quasiparticle fractionalizes into a spinon and a spinless fermion $\chi$ at the QCP (Coleman et al., 2001).

Formally, the Kondo interaction of the Kondo lattice model is decoupled here with a fermionic field (in contrast to the slave boson in standard mean-field theory for the Kondo effect), and the dynamics of this fermion is key for the critical behavior. In contrast to the ideas sketched above, in the approach of Pépin (2005) the Fermi volume does not jump, but evolves continuously through the QCP. Building on a number of phenomenological assumptions on the dynamics and dispersion of the $\chi$ mode, it is possible to describe various properties of $\mathrm{YbRh}_{2} \mathrm{Si}_{2}$, like a $T^{-1 / 3}$ upturn of the specific-heat coefficient at low temperatures which is not reflected in transport measurements. However, the theory of Pépin (2005) is not able to describe the heavy Fermi-liquid state itself, as the $\chi$ fermion cannot condense.

\section{One vs. two transitions}

For all the above approaches, two possibilities arise. Generically, the breakdown of Kondo screening will not occur at the same point as the magnetic QCP which is associated with the formation of long-range order. Therefore the Hertz theory (77) for an antiferromagnetic QPT seems to remain valid. The situation is different when the two QCP coincide (or are in close proximity).

Within the EDMFT model, this happens in the case of two-dimensional magnetic fluctuations: the local susceptibility at the magnetic QCP diverges which drives the Kondo effect critical, leading to a power-law behavior of $M(\omega)$. (In $d=3$ the magnetic transition precedes a possible breakdown of Kondo screening.) Interestingly, the momentum dependence of the critical fluctuations in $\mathrm{CeCu}_{6-x} \mathrm{Au}_{x}$ appears to be two-dimensional (Stockert et al., 1998), which also implies that the self-energy of the electrons is weakly momentum-dependent (Rosch et al., 1997). Thus, the EDMFT model reproduces a number of important aspects of the experimental data on $\mathrm{CeCu}_{6-x} \mathrm{Au}_{x}$, but it remains to understand why the magnetic fluctuations are two-dimensional (no obvious structural reason for this behavior is evident), and whether this fact is generic.

A different scenario, based on the idea of a Landau- 
forbidden transition, has been proposed within the fractionalized Fermi-liquid concept (Senthil, Sachdev, and Vojta, 2005). Provided that the primary transition is the Kondo breakdown (leading to localized $f$ moments on the r.h.s. of the phase diagram in Fig. 5), magnetism can arise as a secondary instability of the $\mathrm{FL}^{*}$ state. The RG flow is similar to that proposed in the scenario of deconfined quantum criticality (Senthil et al., 2004a,b), i.e., an operator which destabilizes the deconfined phase (in our case towards magnetism) is irrelevant at the critical point. A consequence would be the presence of two different energy scales on the magnetic side of the QPT: fluctuations associated to the Kondo effect (loosely speaking Fermi surface fluctuations) exist on a much higher energy scale than magnetic fluctuations; this is accompanied by rather weak magnetism (i.e. an anomalously small ordered moment) close to the QCP. Then, the Néel temperature, $T_{\mathrm{N}}$, will vanish faster upon approaching the QPT than the temperature scale, $T_{\text {coh }}$, at which well-defined quasiparticles appear at the large Fermi surface on the FL side (see Fig 5). A microscopic calculation verifying this proposal is not available to date; a "naive" slaveparticle calculation (which is blind to the mechanism of deconfined criticality) yields two separate critical points (Senthil, Vojta, and Sachdev, 2004).

\section{J. Disorder effects close to quantum phase transitions}

When dealing with real materials, the influence of static or quenched disorder on the properties of a quantum phase transition is an important aspect. Remarkably, the effect of disorder is not completely understood even for classical phase transitions.

In a theoretical description, quenched disorder can occur in different ways: on a microscopic level, e.g., random site energies or bond couplings, or randomly distributed scattering centers are possible. In an orderparameter field theory, disorder usually translates into a random mass term for the order-parameter fluctuations. Importantly, the quantum statistical description of a quantum problem with quenched disorder leads to a $(d+z)$-dimensional field theory with strongly anisotropic correlated disorder because the disorder is frozen in the time direction. In some cases, lattice effects not captured by the field theory can be important, this applies, e.g., to all types of percolation problems. Moreover, disordering a quantum model can lead to random Berry phase terms which have no classical analogue, an example are diluted Heisenberg magnets.

If disorder is added to a system which displays a continuous (classical or quantum) phase transition, obvious questions arise: (i) Will the phase transition remain sharp or become smeared? (ii) Will the critical behavior change? (iii) What happens in the vicinity of the "dirty transition"?

\section{Harris criterion and fixed points}

To answer the first two of the above questions one has to investigate the stability of a critical fixed point with respect to a small amount of disorder. The Harris criterion (Chayes et al., 1986; Harris, 1974) states that disorder will induce qualitative changes (i.e. is a relevant perturbation) if $\nu d<2$, where $\nu$ is the correlation-length exponent. Note that the Harris criterion is identical for classical and quantum phase transitions (i.e., $d$ is not replaced by $d+z$ ) because the disorder is frozen in the time direction. Within the LGW theory (77) of Hertz, $\nu=1 / 2$ for $d+z \geq 4$, therefore disorder is always relevant sufficiently close to the QCP in a system with quenched disorder for $d=2$ and 3 (Harris, 1974; Kirkpatrick and Belitz, 1996b).

Combining results of neutron scattering and thermodynamic measurements, one can use the Harris argument to obtain an order-of-magnitude estimate of the temperature and doping regime where disorder will affect the system. For our argument, we consider a material where a magnetic QCP is reached by doping, e.g. a ternary compound $A B_{1-x} C_{x}$ with a QCP at $x=x_{c}, x_{c}<1 / 2$. On the non-magnetic side of the phase diagram, one can think of the system as consisting of many fluctuating domains of size $\xi$ with a volume $V_{\xi} \sim \xi^{d}$. The number $N_{C}$ of impurities in such a domain is approximately given by $N_{C}=x V_{\xi} / V_{\mathrm{UC}} \approx c x\left(x-x_{c}\right)^{-\nu d}$, where $V_{\mathrm{UC}}$ is the volume of the unit cell and the constant $c \approx$ $\left(x_{0}-x_{c}\right)^{\nu d} V_{\xi}\left(x_{0}\right) / V_{\mathrm{UC}}$ can be estimated from inelastic neutron scattering experiments at a doping $x_{0} \neq x_{c}$. Obviously, $N_{\xi}$ fluctuates statistically with variance $\pm \sqrt{N_{\xi}}$. Therefore the typical fluctuations $\delta x$ of the doping $x$ are of the order $\delta x \approx x_{c} / \sqrt{N_{\xi}} \approx \sqrt{x_{c} / c}\left(x-x_{c}\right)^{\nu d / 2}$. The Harris criterion is equivalent to the statement that disorder is relevant if $\delta x>\left|x-x_{c}\right|$ or

$$
\sqrt{x_{c} / c}>\left|x-x_{c}\right|^{1-\frac{\nu d}{2}}
$$

If $\nu d<2$, disorder changes the critical behavior for $\left|x-x_{c}\right|<\delta x^{*} \approx\left(x_{c} / c\right)^{\frac{1}{2-\nu d}}$. This doping scale $\delta x^{*}$ can be translated into a temperature scale $T^{*}$ below which disorder changes the thermodynamics at the QCP, e.g., by using the $x$ dependence of the ordering temperature or of other relevant crossover scales. (However, an orderof-magnitude estimate along these lines for $\mathrm{CeCu}_{6-x} \mathrm{Au}_{x}$ with a QCP at $x_{c}=0.1$ turns out to be inconclusive, but suggests that disorder could be important in the experimentally relevant regime.)

What will happen if quenched disorder is relevant sufficiently close to the QCP? Recent work has shown that three possibilities exist: (a) Disorder leads to a new conventional (finite-disorder) critical point, with powerlaw behavior and exponents fulfilling the Harris criterion, $\nu d \geq 2$. An example is the rung-diluted bilayer Heisenberg magnet in $d=2$ (Sknepnek, Vojta, and Vojta, 2004). (b) Disorder leads to a so-called infinitedisorder fixed point. Here, the dynamics is extremely slow, $\ln \xi_{\tau} \sim \xi^{\mu}$ (replacing the conventional $\xi_{\tau} \sim \xi^{z}$ ), 
and the statistical distributions of observables become very broad. Such a behavior has been established for the random quantum Ising model in $d=1$ (see Sec. III.J.2). (c) Disorder can destroy the sharp transition, replacing it by a smooth crossover. This interesting scenario is relevant for certain metallic magnets and will be discussed in Sec. III.J.3.

Independent of the fate of the phase transition point itself, the third of the above questions is still to be answered: What happens in the vicinity of the transition? Interestingly, even at some distance from the QCP, the system can show power-law behavior (e.g. as a function of temperature) with non-universal exponents. These socalled Griffiths effects are discussed in the next section.

\section{Rare regions and quantum Griffiths singularities}

Disorder in a magnet usually suppresses the ordering tendency and thus changes the location of the phase transition. In a parameter regime where the clean system would order but the disordered does not, one will find (arbitrarily large) regions that are accidentally devoid of impurities, and hence show local order, with a small but non-zero probability that usually decreases exponentially with the size of the region. These static disorder fluctuations are known as "rare regions", and the orderparameter fluctuations induced by them as "local moments" or "instantons". Since they are weakly coupled, and flipping them requires to change the order parameter in a whole region, the local moments have very slow dynamics. Griffiths (1969) was the first to show that rare regions lead to a non-analytic free energy in the whole region between the transition points of the clean and disordered system, known as the Griffiths (or GriffithsMcCoy) region. A review has been recently given by Vojta (2006b).

In generic classical systems Griffiths effects are weak, since the singularity in the free energy is only an essential one. Near quantum phase transitions Griffiths singularities are enhanced compared to the classical case as disorder is frozen in the time direction. Interestingly, the three cases listed above regarding the fate of the transition yield different quantum Griffiths behavior as well (Vojta, 2006b): (a) In the vicinity of a finite-disorder fixed point the Griffiths effects lead to weak exponential corrections. (b) For infinite-disorder fixed points Griffiths effects are strong, and observables display powerlaw singularities with continuously varying exponents. (c) If the rare regions become static, the transition is smeared, and conventional Griffiths behavior does not exist. Griffiths singularities occur in principle also on the ordered side of a QCP, but their signatures are much weaker. Notably, the cases (a/b/c) corresponds to situations where the rare regions are below/at/above the lower-critical dimension of their ordering transition (Vojta and Schmalian, 2005). In the following we sketch the physics of situation (b) which has been thoroughly inves- tigated for spin models. Situation (c) which is relevant for the damped order-parameter dynamics of metals is discussed in Sec.III.J.3.)

The random Heisenberg and transverse-field Ising models have been studied in detail in $d=1$, but more recently also in higher dimensions (Fisher 1995, Motrunich et al. 2000, Pich et al. 1998, Senthil and Sachdev 1996). A transparent physical picture emerges from a real-space RG analysis (Fisher, 1995; Ma, Dasgupta, and Hu, 1979; Motrunich et al., 2000). For a strongly disordered AFM Heisenberg chain, $H=\sum J_{i j} \mathbf{S}_{i} \mathbf{S}_{j}$, the decimation RG scheme proceeds as follows: In each step, the strongest bond is eliminated (i.e. frozen as a singlet), which induces a new coupling between the adjacent spins via secondorder perturbation theory. The RG procedure follows the flow of the distribution of couplings $P(J)$ upon successive elimination of spins and bonds. The renormalization scheme is based on a strong-coupling expansion and perturbation theory, it is valid if the strongest coupling is typically much larger than neighboring couplings, i.e., if the distribution $P(J)$ is very broad. For the Heisenberg chain a typical initial distribution of disorder gets broader and broader: it flows towards an infinite-randomness fixed point, corresponding to a so-called random-singlet phase, and the method described above is asymptotically exact. For the transverse-field Ising chain, where a similar scheme can be applied, the two stable phases are conventional, but the flow to infinite randomness occurs at the zero-temperature phase transition point. The critical dynamics at such an infinite-randomness fixed point turns out to be extremely slow, with $\ell n \xi_{\tau} \propto \xi^{\mu}$ (so-called activated scaling), and the distributions of macroscopic observables become infinitely broad.

If the QPT is controlled by such an infinite-randomness fixed point [situation (b)] the following picture emerges (Fig. 6): On the paramagnetic side of the phase diagram, a distribution of magnetic domains induces a distribution of local susceptibilities $\chi_{i}$ or typical energies $\Delta_{i} \sim 1 / \chi_{i}$ with probabilities $P\left(\Delta_{i}<\Delta\right) \sim \Delta^{d / z^{\prime}}$, where $d$ is the dimension. The smallest possible energy $\Delta_{\min }(L)$ in a region of size $L$ is therefore given by $P\left(\Delta_{i}<\Delta_{\text {min }}\right) \sim 1 / L^{d}$ (as there are $L^{d}$ sites within this region) and $\Delta_{\min } \sim L^{-z^{\prime}}$. In this sense, $z^{\prime}$ is a dynamical critical exponent. However, one should keep in mind that minimal, typical, and average energies can be very different in this Griffiths regime. Note that the characteristic low-energy scale of a domain consisting of $N$ spins, e.g. the tunnel splitting between different magnetic configurations, is exponentially small in $N$. The relevant domain sizes $N \sim \ln \Delta$ are therefore rather small, and the size of domains enters most physical properties only logarithmically. From the distribution of energies one finds for the specific-heat coefficient and the average susceptibility

$$
\chi \sim c_{V} / T \sim T^{d / z^{\prime}-1} .
$$

The divergence of the average non-linear susceptibility is even stronger, $\chi_{n l}^{(3)} \sim T^{d / z^{\prime}-3}, \chi_{n l}^{(3)}$ can diverge even if $\chi$ is regular. As the typical size of domain is of order $\ell n N$ 

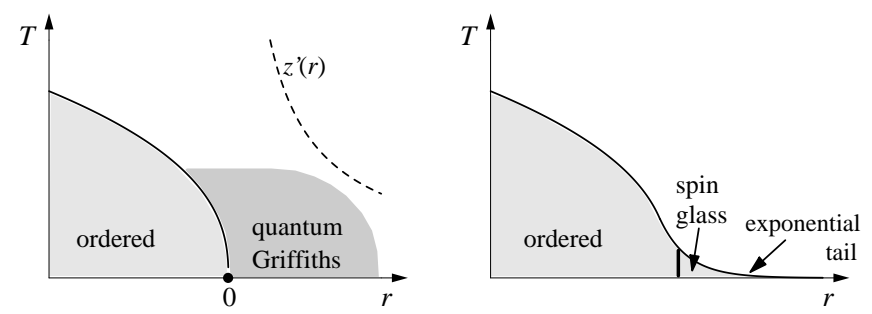

FIG. 6 Schematic phase diagrams near a QCP in the presence of quenched disorder. Left: infinite-randomness fixed point with Griffiths region [situation (b)]. In the Griffiths region, thermodynamic quantities display power-law singularities in a finite region around the QCP. The dashed line indicates the divergence of the dynamical exponent $z^{\prime}$ upon approaching the QCP. Right: smeared phase transition [situation (c)], with exponentially small transition temperature on the disordered side of the clean system $(r>0)$.

and therefore small, the leading temperature dependence of the order-parameter susceptibility and the static susceptibility is typically the same. The exponents $d / z^{\prime}$ in the Griffiths region are non-universal as they depend on microscopic details and the distance $r$ from the QCP. For the infinite-randomness fixed points one finds $z^{\prime} \sim r^{-\nu \Psi}$, with universal exponents $\Psi$ and $\nu$. (For numerical values see Motrunich et al., 2000; Pich et al., 1998.) This characteristic dependence of the Griffiths exponents on the distance to the QCP is in our opinion the most important signature of the quantum Griffiths scenario. Experiments on $\mathrm{UCu}_{5-x} \mathrm{Pd}_{x}$ may possibly provide an example of this dependence (Vollmer et al., 2000).

Numerical simulations (Motrunich et al., 2000; Pich et al., 1998) suggest that infinite-randomness fixed points may not be restricted to systems in $d=1$, raising the possibility that exotic critical behavior dominated by rare regions may be common to certain quenched-disorder quantum systems, in particular those with Ising symmetry. Recent investigations of $2 \mathrm{~d}$ diluted antiferromagnets with Heisenberg symmetry indicate conventional critical behavior [situation (a)], and in addition an interesting interplay of quantum and geometric criticality at the percolation threshold (Sandvik, 2002; Sknepnek, Vojta, and Vojta, 2004).

Castro Neto, Castilla, and Jones (1998); Castro Neto and Jones (2000) have proposed that Griffiths singularities (and the related Kondo disorder scenario) can explain the anomalous behavior in certain strongly disordered heavy-fermion systems. Indeed, de Andrade et al. (1998) fitted $C / T$ and $\chi$ of a number of systems over a certain temperature range with Eq. (148), e.g., $\mathrm{Th}_{1-x} \mathrm{U}_{x} \mathrm{Pd}_{2} \mathrm{Al}_{3}, \mathrm{Y}_{1-x} \mathrm{U}_{x} \mathrm{Pd}_{3}$ or $\mathrm{UCu}_{5-x} \mathrm{Pd}_{x}$. More recently, the low-field ac susceptibility of $\mathrm{Ce}\left(\mathrm{Ru}_{1-x} \mathrm{Rh}_{x}\right)_{2} \mathrm{Si}_{2}$ was found to exhibit $B / T$ scaling compatible with a quantum Griffiths scenario (Tabata et al., 2004), while ${ }^{29} \mathrm{Si}$ NMR data on $\mathrm{CePtSi}_{1-x} \mathrm{Ge}_{x}$ near a magnetic instability appear to dis- agree with this scenario (Young et al., 2004).

As we will discuss in the next section, in metallic systems with Ising symmetry the quantum tunneling of the magnetic domains - which is at the heart of the quantum Griffiths effect - is prohibited at lowest temperatures by the coupling to the fermions (Millis, Morr, and Schmalian, 2002). Whether the characteristic power laws are nevertheless observable in a certain temperature regime may depend on non-universal details and is not completely clear (Castro Neto, Castilla, and Jones, 1998; Millis, Morr, and Schmalian, 2002).

Miranda and Dobrosavljević (2001) succeeded in calculating Griffiths singularities close to a metal-insulator transition in a strongly correlated electron system. The authors attacked the challenging problem to describe both the formation of localized magnetic moments and the metal-insulator transition using the so-called "statistical dynamical mean-field" (SDMFT) approximation. These developments have been recently reviewed by Miranda and Dobrosavljević (2005).

\section{Effects of rare regions on metallic QPT}

How do disorder and rare regions influence the QCP in a metal? In general, the situation is different from insulators due to the overdamped order-parameter dynamics. Early approaches, e.g., by Narayanan et al. (1999) who used a RG formulation in terms of the order parameter (Kirkpatrick and Belitz, 1996a) and found run-away flow to strong disorder, remained inconclusive regarding the nature of the transition. By now, only a few results are available, and the answer may depend on the orderparameter symmetry.

Remarkably, for a metallic antiferromagnet with Ising symmetry the effects of disorder are so strong that a direct transition from the antiferromagnetic to a paramagnetic (Griffiths) phase is prohibited [situation (c)] (Vojta, 2003b). Generically, a spin-glass phase emerges at the lowest temperatures - for the specific model considered by Vojta (2003b) the antiferromagnetic transition is smeared. This effect can be understood starting from the properties of a single rare region, a small antiferromagnetic domain, in the (nominally) paramagnetic phase. As disorder is frozen in time direction, a rare region in space translates into a rod-like object in space-time, which has an effective $1 / \tau^{2}$ interaction arising from Landau damping, i.e., the $|\omega|$ term in Eq. (78). The effective onedimensional Ising model for each rare region displays an ordered phase due to this long-range interaction, in other words, isolated rare regions will develop static order. In this situation, quantum Griffiths behavior (as described above) does not exist as there is no quantum tunneling. Isolated rare regions are coupled weakly by a RKKY interaction with random sign which leads to the formation of a classical spin glass (or rather "domain" or "cluster" glass), with the transition temperature being exponentially suppressed by the distance from the quantum crit- 
ical point, see Fig. 6. It is not known how the spin glass and antiferromagnetic phases merge; a likely scenario is a first-order phase transition.

For continuous symmetries the situation is less clear. The rare-region physics of Vojta (2003b), applied to an itinerant magnet with Heisenberg (instead of Ising) symmetry, has been shown (Vojta and Schmalian, 2005) to give rise to power-law Griffiths singularities near criticality, and the QCP is then likely of infinite-randomness type. However, Dobrosavljević and Miranda (2005) have argued that the coupling between the rare regions can also lead to a "freezing" of magnetic domains at very low $T$, destroying the Griffiths physics. Again this is expected to lead to a glassy state for $T \rightarrow 0$.

\section{Metallic quantum glasses}

Strong disorder can lead to the formation of itinerant glass phases, with glassiness in the spin sector (metallic spin glasses, SG) or the charge sector (electron glasses). We will not discuss the physics of metallic glasses in detail and refer the reader to the theory reviews of Sachdev and Read (1996) and Miranda and Dobrosavljević (2005) (for an experimental introduction see Mydosh 1993), and restrict the discussions here to a few short remarks.

Unfortunately, not much is known theoretically about metallic spin glasses (SG) in 3d systems. While the description of the ordered phase of metallic AFM or FM within Fermi-liquid theory is straightforward, a theory of a metallic SG has been developed mainly for infiniterange models (Sachdev, Read, and Oppermann, 1995; Sengupta and Georges, 1995). Close to the QCP of a metallic SG, one can expect both non-Fermi liquid behavior due to critical fluctuations and Griffiths singularities (see section above and Sachdev 1998). Surprisingly, the QCP of a metallic SG is very similar to the $d=3$, $z=2$ theory of Hertz (77) (Sachdev, Read, and Oppermann, 1995; Sengupta and Georges, 1995): the authors predict at the QCP a $\sqrt{T}$ correction to the specific-heat coefficient and a $T^{3 / 2}$ law in the temperature dependence of the resistivity. We note that these mean-field theories do not satisfy hyperscaling. The actual situation for realistic spin glasses remains an open problem; an analysis of fluctuations around the mean-field solution leads to a runaway flow to strong coupling.

Glassy behavior in the charge sector has long been investigated in disordered insulators, where studies have concentrated on the physics of the Coulomb glass. Recent work has discussed the zero-temperature melting of such a glassy phase due to quantum fluctuations (Pastor and Dobrosavljevic, 1999). A mean-field theory for the transition from a Fermi liquid to an electron glass (Dalidovich and Dobrosavljevic, 2002) predicts a $T^{3 / 2}$ behavior in the resistivity; corrections to the mean-field behavior have not been determined.

\section{FERMI-LIQUID INSTABILITIES AT QUANTUM PHASE TRANSITIONS: EXPERIMENT}

As already mentioned in the Introduction, there are several material classes where non-Fermi-liquid behavior is observed. In the spirit of this review, we will focus on quantum phase transitions and will not discuss single-ion NFL scenarios (see Sec. I.D). Suffice it to mention that, historically, the first $4 f$-electron NFL system was $\mathrm{U}_{x} \mathrm{Y}_{1-x} \mathrm{Pd}_{x}$ with $x \approx 0.2$, where the observed specific-heat behavior $C / T \sim \ln \left(T_{0} / T\right)$ was interpreted by Seaman et al. (1991) as arising from the two-channel quadrupolar Kondo effect, while essentially similar data were suggested by Andraka and Tsvelik (1991) to signal a quantum phase transition. Further work by the Maple group underlined the metallurgical and disorderrelated issues with this material by a comparative study of $\mathrm{U}_{x} A_{1-x} \mathrm{Pd}_{x}$, with $A=\mathrm{Y}$, La and Sc (Gajewski, Chau, and Maple, 2000). A recent report indicated some scaling of the dynamical susceptibility $\chi(\omega, T)$ (Wilson et al., 2005) although the scatter of the data was substantial.

From the beginning, there has been a lot of discussion about Kondo disorder leading to NFL behavior. Here $\mathrm{UCu}_{5-x} \mathrm{Pd}_{x}$ was in focus (Bernal et al., 1995), which was the first material where a new type of anomalous scaling of the (wavevector-integrated) susceptibility $\chi(\omega, T)$ was observed (Aronson et al., 1995). $\mathrm{UCu}_{5-x} \mathrm{Pd}_{x}$ has also been a candidate for the Griffiths scenario (Sec. III.J.2) (de Andrade et al., 1998) as corroborated by a dependence of the temperature exponents of $\chi$ and $C / T$, Eq. (148), on the distance from the quantum critical points at $x=1$ and 1.5 (Vollmer et al., 2000). However, one has to bear in mind that the detailed behavior of this system is strongly affected by the $\mathrm{Cu} / \mathrm{Pd}$ site interchange, the amount of which depends on annealing (Booth et al., 2002; MacLaughlin et al., 2006). For instance, Booth et al. (2002) reported - instead of a power law - a logarithmic temperature dependence of $C / T$ in annealed $\mathrm{UCu}_{4} \mathrm{Pd}$.

Leaving these matters aside, we now briefly describe the main focus of this section, i.e., experiments on magnetic QPT in intermetallic rare-earth and transitionmetal alloys and compounds. (Superconductivity will be discussed in so far as it is relevant to quantum criticality.) Rather than trying to be exhaustive in treating every system for which NFL behavior near a magnetic QPT has been claimed, we will discuss mostly systems that have been characterized thoroughly. These comprise heavyfermion systems, e.g., $\mathrm{CeCu}_{6-x} \mathrm{Au}_{x}, \mathrm{YbRh}_{2} \mathrm{Si}_{2}$, and the Ce-115 compounds, as well as transition metal compounds such as $\mathrm{MnSi}$ and $\mathrm{ZrZn}_{2}$. A comprehensive review of NFL behavior in a large variety of heavy-fermion systems has been given by Stewart $(2001,2006)$. The issue of (one or several) QPT in cuprate high-temperature superconductors and other transition-metal oxides is beyond the scope of this review. Of course, the linear resistivity $\rho(T)$ in cuprates at optimal doping constitutes an early manifestation of NFL behavior (cf. the marginal 
Fermi-liquid phenomenology of Varma et al. 1989).

In discussing QPT, two generic cases have to be distinguished. The prototype is a continuous, e.g., secondorder transition between two ground states tuned by a non-thermal parameter. If the transition is discontinuous (first order), the evolution of the length and time scales fluctuations will be limited. An interesting case arises when a line of first-order transitions terminates in a critical endpoint and if this critical point can be driven to zero temperature by another tuning parameter, leading to a quantum critical end point. This might be the case for $\mathrm{Sr}_{3} \mathrm{Ru}_{2} \mathrm{O}_{7}$ where tuning parameters are magnetic field and the field orientation (Grigera et al., 2001; Millis et al., 2002).

\section{A. Quantum critical behavior in heavy-fermion systems}

In general, Fermi-liquid theory has been very successful in describing the low-temperature behavior of metals with strong electronic correlations, including many heavy-fermion systems (HFS). These materials often comprise a regular sublattice of $4 f$ or $5 f$ atoms, notably $\mathrm{Ce}, \mathrm{Yb}$, or U. Under certain conditions, a crossover of the magnetic behavior occurs with decreasing temperature $T$, from that of a collection of "free" localized $4 f$ or $5 f$ magnetic moments (subjected to the crystalline electric field and to spin-orbit interaction) coupled weakly to the conduction electrons, to low- $T$ local singlets where the localized moment is screened completely by the conduction electrons by virtue of the Kondo effect, Sec. II.F.

The energy gain of singlet formation $T_{\mathrm{K}} \sim$ $D \exp \left(-1 / N_{0} J\right)$ sets the temperature scale where this crossover occurs, i.e., the Kondo temperature $T_{\mathrm{K}}$. Here $N_{0}$ is the (unrenormalized) conduction-electron (c) density of states at the Fermi level and $J$ is the exchange constant between $c$ and $f$ electrons. The onset of a coherent state leading to a Fermi liquid at usually still lower temperatures is mostly signaled by a maximum in the electrical resistivity $\rho(T)$. Although there exists a lot of discussion on the onset of coherence at a coherence temperature $T_{\text {coh }}$ thus defined, its relation to $T_{\mathrm{K}}$ remains under debate, see Sec. II.F.2. Here we use for simplicity $T_{\mathrm{K}}$ as a measure of the energy gain due to singlet formation, keeping in mind that $T_{\mathrm{K}}$ in HFS might be modified by interactions with respect to the single-ion Kondo temperature in dilute magnetic alloys. Experimentally, this $T_{\mathrm{K}}$ is often determined through fits of the intermediatetemperature data for $C(T)$ or $\chi(T)$ to single-impurity results. (Frequently the symbol $T^{*}$ is used for the characteristic temperature of a Kondo lattice, instead of $T_{\mathrm{K}}$.)

At sufficiently low $T \ll T_{\mathrm{K}}$, Fermi-liquid (FL) properties are observed in many HFS with a very large effective mass $m^{*}$ derived from the huge linear specific-heat coefficient $\gamma=C / T$ and a correspondingly large Pauli susceptibility, both being only weakly dependent on $T$. The electrical resistivity of a FL exhibits a contribution $\Delta \rho=A T^{2}$ arising from electron-electron collisions, see
Sec. II.E.3. The phenomenological correlations $\gamma \sim \chi$ (Fisk, Ott, and Aeppli, 1987) and $A \sim \gamma^{2}$ (Kadowaki and Woods 1986, see Sec. II.E.3) observed approximately for different HFS, do suggest the validity of the FL description. The Wilson ratio $R=(\chi / \gamma)\left(\pi^{2} k_{B}^{2} / \mu_{0} \mu_{\text {eff }}^{2}\right)$ deviates from the free-electron value $R=1$ (Fisk, Ott, and Aeppli, 1987). The observed values of $R \sim 2$ to 5 can be accounted for in the frame of FL theory by a negative Landau parameter $F_{0}^{a}$ of the order of -0.5 to -0.8 , see Secs. II.B and II.F.2, Eq. (44). The problem of proving or disproving FL behavior in HFS lies in the low energy scale set by the Kondo temperature $T_{\mathrm{K}} \sim 10$ to $100 \mathrm{~K}$ compared to conventional metals where $T_{F} \sim 10^{4}$ to $10^{5} \mathrm{~K}$. The first heavy-fermion system discovered was $\mathrm{CeAl}_{3}$, with a $\gamma$ coefficient of $\sim 1.5 \mathrm{~J} / \mathrm{molK}^{2}$ (Andres, Graebner, and Ott, 1975). The interest in these materials increased tremendously when Steglich et al. (1979) reported superconductivity in $\mathrm{CeCu}_{2} \mathrm{Si}_{2}$, with the heavy quasiparticles being responsible for the superconductivity. The early work on heavy-fermion systems has been reviewed by Grewe and Steglich (1991).

The competition between on-site Kondo interaction, quenching the localized magnetic moments, and intersite RKKY interaction between these moments allows for non-magnetic or magnetically ordered ground states in HFS. In the Doniach picture (Doniach, 1977), this competition is governed by a single parameter, namely the effective exchange constant $J$ between conduction electrons and local moments, which enters the characteristic energy scales $T_{\mathrm{K}}$ and $T_{\mathrm{RKKY}} \sim J^{2} N_{0}$ ) for Kondo and RKKY interactions, respectively (see Sec. II.F.2). The strength of the exchange interaction is usually tuned by composition or pressure. In addition, a large magnetic field can suppress Kondo screening. Owing to the extremely strong dependence of the Kondo energy scale on the interatomic distance $d$, which arises from the exponential dependence of $T_{\mathrm{K}}$ on $J$, volume changes are often the dominant effect in producing the magnetic-nonmagnetic transition if isoelectronic constituents are substituted against each other.

Many HFS show some kind of static magnetic order with often very tiny ordered moments (of the order of or smaller than $10^{-2} \mu_{B}$ ). A particularly intriguing example is $\mathrm{CeAl}_{3}$, long considered the archetypal HFS without magnetic order. However, $\mathrm{CeAl}_{3}$ has been shown to order antiferromagnetically when produced in singlecrystalline form (Lapertot et al., 1993). This is probably due to subtle structural differences, e.g., strains, between polycrystals and single crystals. An inhomogeneous distribution of Kondo temperatures was inferred from NMR measurements in this material (Gavilano, Hunziker, Ott, 1995).

This example shows that disorder can have a decisive influence on the low-temperature properties even in nominally stoichiometric HFS samples. For nonstoichiometric substitutional alloys one can distinguish between substitution of the $4 f$ or $5 f$ site and on the ligand site. In both cases, a pronounced effect may be an- 
ticipated. In the former case, replacement of Ce or $\mathrm{U}$ by a non-magnetic atom in a otherwise stoichiometric HFS might lead to the formation of a Kondo hole. In the latter case, replacement of the ligand atom around a given $\mathrm{Ce}$ or $\mathrm{U}$ site might change the hybridization and hence of the local $T_{\mathrm{K}}$. In general, predictions are difficult as to which effect will be stronger in a given system. Although much work on QPT that were tuned by composition of substitutional alloys has been done, stoichiometric compounds avoiding disorder are preferable, as many of the complications, theoretically anticipated for samples with sizeable disorder (see Sec. III.J), will be absent. Alternatively, different tuning parameters should be employed to check the role of disorder, as has been done for $\mathrm{CeCu}_{6-x} \mathrm{Au}_{x}$.

\section{1. $\mathrm{CeCu}_{6-x} \mathrm{Au}_{x}$ and $\mathrm{CeCu}_{6-x} \mathrm{Ag}_{x}$}

$\mathrm{CeCu}_{6}$ has been established as a HFS showing no longrange magnetic order down to the range of $\sim 20 \mathrm{mK}$ (Amato et al., 1987; Ōnuki and Komatsubara, 1987). $\mathrm{CeCu}_{6}$ crystallizes in the orthorhombic Pnma structure and undergoes an orthorhombic-monoclinic distortion around $200 \mathrm{~K}$ (Gratz et al., 1987). The change of the orthorhombic angle is only small $\left(\sim 1.5^{\circ}\right)$. In order to avoid confusion, we always use the orthorhombic notation for the direction of the lattice vectors. $\mathrm{CeCu}_{6}$ exhibits a pronounced magnetic anisotropy with the magnetization ratios along the three axes $M_{c}: M_{a}: M_{b} \approx 10: 2: 1$ at low $T$ (Amato et al., 1987). Schuberth et al. (1995) have extended the measurements of the specific heat $C$ down to $10 \mathrm{mK}$ and of the magnetic susceptibility $\chi$ to even below $1 \mathrm{mK}$. Their analysis of $\chi$ at very low $T$ (after subtraction of an impurity contribution attributed to Gd) suggests magnetic order around $5 \mathrm{mK}$. This is backed by NQR measurements which likewise hint at (possibly nuclear) magnetic order (Pollack et al., 1995). Direct evidence for magnetic order below $2 \mathrm{mK}$ was found in the $a c$ magnetic susceptibility and thermal expansion (Tsujii et al., 2000). $\mu \mathrm{SR}$ measurements have put an upper limit for a static moment of $10^{-2}$ to $10^{-3} \mu_{B} /$ Ce-atom (depending on the assumption of long-range magnetic vs. spin-glass order) above $40 \mathrm{mK}$ (Amato et al., 1993).

Although $\mathrm{CeCu}_{6}$ does not order magnetically above $5 \mathrm{mK}$, the expectation $C / T \approx$ const for a $\mathrm{FL}$ is not met very well (see Fig. 7). The single-ion Kondo model with $T_{\mathrm{K}}=6.2 \mathrm{~K}$ does not fit the data below $\sim 0.4 \mathrm{~K}$ (Schlager et al., 1993). Instead $C / T$ increases slightly towards low $T$ which might be a precursor of the 5 -mK order. On the other hand, the $T^{2}$ dependence of the electrical resistivity is rather well obeyed between 40 and $200 \mathrm{mK}$ (Amato et al., 1987). Antiferromagnetic fluctuations were observed in inelastic neutron scattering by peaks in the dynamic structure factor $S(Q, \omega)$ for energy transfer $\hbar \omega=$ $0.3 \mathrm{meV}$ at $\mathbf{Q}=\left(\begin{array}{lll}1 & 0 & 0\end{array}\right)$ and $\left(\begin{array}{ll}0 & 1 \pm 0.15\end{array}\right)$ (Rossat-Mignod et al., 1988). The rather large widths of these peaks correspond to correlation lengths extending roughly only to the nearest Ce neighbors. These correlations vanish at

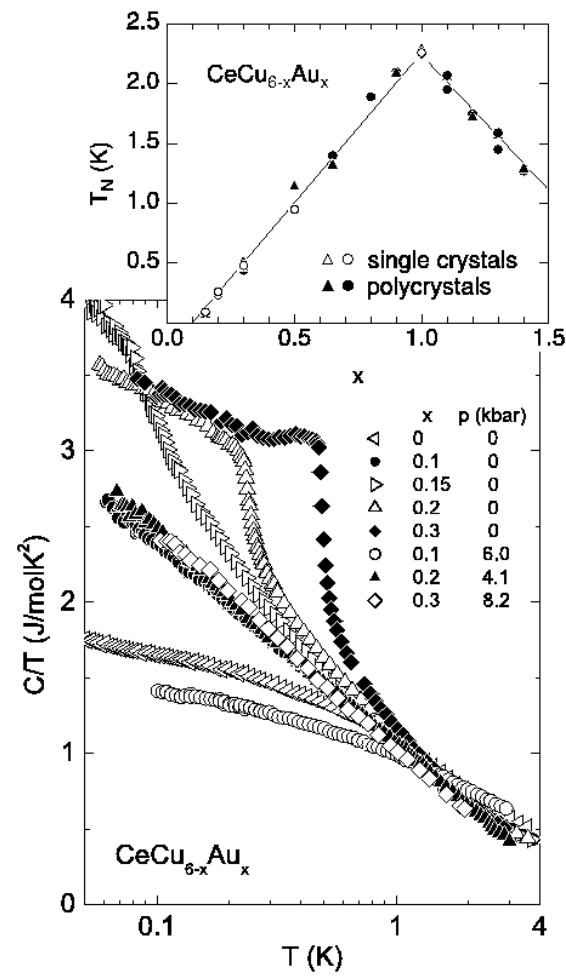

FIG. 7 Specific heat $C$ of $\mathrm{CeCu}_{6-x} \mathrm{Au}_{x}$ in the vicinity of the QPT plotted as $C / T$ vs. temperature $T$ (logarithmic scale). Application of hydrostatic pressure at the respective critical value $p_{c}$ shifts $C / T$ of the antiferromagnetic samples $x=0.2$ and 0.3 towards NFL behavior for $x=0.1$ at ambient pressure. From v. Löhneysen et al., 1996a, 1998a. The inset shows the Néel temperature $T_{\mathrm{N}}$ of $\mathrm{CeCu}_{6-x} \mathrm{Au}_{x}$ vs. Au concentration $x$ as determined from specific heat (triangles) and magnetic susceptibility (circles). From Pietrus et al., 1995.

a field of $\approx 2 \mathrm{~T}$ applied along the easy $c$ direction, also associated with a shallow maximum at $2 \mathrm{~T}$ in the differential magnetic susceptibility $d M / d B$ at very low $T$ (v. Löhneysen, Schlager, and Schröder, 1993). This maximum has been identified with the "metamagnetic transition" in loose analogy to the metamagnetic transition in strongly anisotropic antiferromagnets.

Upon alloying with $\mathrm{Au}$ the $\mathrm{CeCu}_{6}$ lattice expands while retaining - in fact: stabilizing - the orthorhombic (at room temperature) Pnma structure (Pietrus et al., 1995). Thus the hybridization between Ce $4 f$ electrons and conduction electrons, and hence $J$, decrease, leading to a stabilization of localized magnetic moments which can now interact via the RKKY interaction. The result is incommensurate antiferromagnetic order in $\mathrm{CeCu}_{6-x} \mathrm{Au}_{x}$ beyond a threshold concentration $x_{c} \approx 0.1$. This was first inferred from sharp maxima in the specific heat $C(T)$ and magnetization $M(T)$ (Germann et al., 1988) and confirmed by neutron scattering (Chattopadhyay et al., 1990; Schröder et al., 1994; v. Löhneysen et al., 1998b).

For $0.1 \leq x \leq 1$ where Au exclusively occupies the $\mathrm{Cu}(2)$ position in the $\mathrm{CeCu}_{6}$ structure, the Néel temperature $T_{\mathrm{N}}$ varies linearly with $x$ (Fig. 7 ). For the stoichio- 

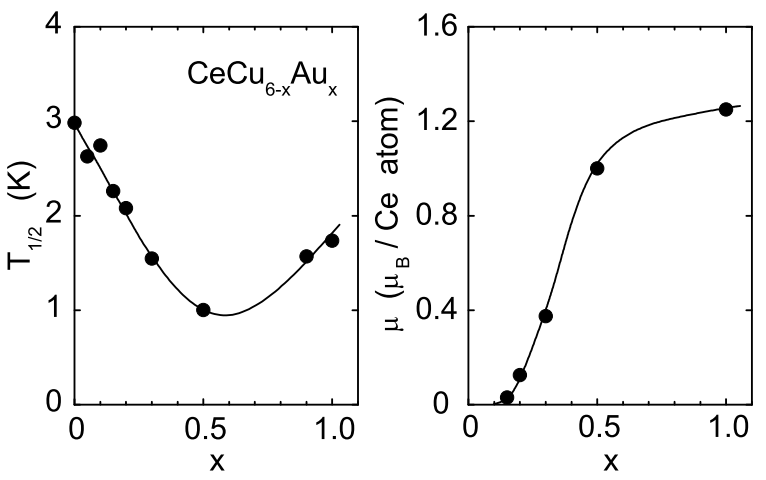

FIG. 8 Temperature $T_{1 / 2}$ and staggered moment $\mu$ for $\mathrm{CeCu}_{6-x} \mathrm{Au}_{x}$. Left: Concentration dependence of the temperature $T_{1 / 2}$ where the magnetic entropy reaches $0.5 R \ell n 2$, obtained from a temperature integration of the specific-heat data. The decrease in $T_{1 / 2}$ with $x$ for $x<0.5$ might reflect the decrease in $T_{\mathrm{K}}$ inferred an analysis of the high-field specific heat in the frame of a single-ion Kondo model (v. Löhneysen et al., 1996a), while the larger $T_{1 / 2}$ at $x=1$ likely results from the strong AF order. Right: $x$ dependence of the staggered moment per Ce ion extracted from elastic neutron scattering. From v. Löhneysen et al., 1998b. Lines are to guide the eye.

metric compound $\mathrm{CeCu}_{5} \mathrm{Au}$ a complex magnetic phase diagram has been mapped out (Paschke et al., 1994b). Beyond $x=1, T_{\mathrm{N}}$ decreases again. This is due to a subtle change within the orthorhombic structure: for $x<1$ the lattice parameters $a$ and $c$ increase while $b$ decreases with growing $\mathrm{Au}$ content, whereas for $x>1$ all $a, b$, and $c$ increase (Pietrus et al., 1995). The orthorhombicmonoclinic transition is quickly suppressed with increasing $x$ and vanishes around $x=0.14$ (Grube et al., 1999). The order parameter, i.e., the staggered magnetic moment per Ce atom as extracted from elastic neutron scattering data (v. Löhneysen et al., 1998b), is shown in Fig. 8. The pronounced rise between $x=0.3$ and 0.5 may be related to the change of the magnetic ordering wavevector occurring in the same $x$ range.

Fig. 7 shows specific-heat data for concentrations in the vicinity of the critical concentration $x_{c} \approx 0.1$ plotted as $C / T$. The Néel temperature $T_{\mathrm{N}}$ manifests itself as a sharp kink in $C / T$ which becomes less pronounced as $T_{\mathrm{N}}$ decreases. It is, however, still clearly visible for $x=0.15$ where $T_{\mathrm{N}}=0.080 \mathrm{~K}$ as confirmed by a maximum in the susceptibility. For $x=0.1$ we observe the non-Fermiliquid behavior $C / T=a \ell n\left(T_{0} / T\right)$, with $a=0.578 \mathrm{~J} / \mathrm{mol}$ $\mathrm{K}^{2}$ and $T_{0}=6.2 \mathrm{~K}$, between $0.06 \mathrm{~K}$ and $\sim 2.5 \mathrm{~K}$, i.e., over almost two decades in temperature, rendering it one of the best examples of a logarithmic divergence of $C / T$ for $T \rightarrow 0$. (The positive deviations above $2.5 \mathrm{~K}$ can be attributed to phonon and crystal-field contributions to $C$.) Concerning the NFL behavior at $x_{c}$ it is important to verify that it does not arise from some inhomogeneity of the alloys, i.e., a distribution of magnetic ordering temperatures. A muon spin relaxation ( $\mu \mathrm{SR})$ study has shown that there is no ordered magnetic moment in a $x$ $=0.1$ sample, with the detection limit $\mu<10^{-3} \mu_{B} /$ Ce- atom (Amato et al., 1995). Likewise, a wide distribution of Kondo temperatures appears to be unlikely (Bernal et al., 1996).

The specific-heat data can be temperature-integrated to obtain the entropy, where the dominant lowtemperature contribution arises from the Ce moments. As a free moments (with effective spin $1 / 2$ ) would give an entropy of $R \ell n 2$, it is convenient to define a temperature, $T_{1 / 2}$, where the entropy reaches $0.5 R \ell n 2$ - this defines a scale which is a measure of the (Kondo) temperature, below which the moments are screened. The $x$ dependence of $T_{1 / 2}$ for $\mathrm{CeCu}_{6-x} \mathrm{Au}_{x}$ is shown in Fig. 8 . Importantly, $T_{1 / 2}$ does not vanish at $x_{c}$. Its decrease with $x$ can be essentially understood from the weakening of the Kondo effect because of the lattice expansion upon alloying $\mathrm{Au}$; the increase of $T_{1 / 2}$ towards $x=1$ is likely due to the quenching of the moments by the ordered antiferromagnetism.

As expected from the correlation between molar volume and $T_{\mathrm{N}}$ discussed above, $T_{\mathrm{N}}$ of $\mathrm{CeCu}_{6-x} \mathrm{Au}_{x}$ decreases under hydrostatic pressure $p$ (Bogenberger and v. Löhneysen, 1995; Germann and v. Löhneysen, 1989; Sieck et al., 1997). The Néel temperature (again as determined from the inflection point of $C(T)$ above the maximum) decreases linearly with increasing $p$ for $x=0.3$. For $x=0.2$ a linear $T_{\mathrm{N}}(p)$ decrease is also compatible with the data. $T_{\mathrm{N}} \approx 0$ is reached for $7-8 \mathrm{kbar}$ and 3.2 - $4 \mathrm{kbar}$ for $x=0.3$ and 0.2 , respectively. At these pressures both alloys exhibit NFL behavior with, surprisingly, the same coefficients $a$ and $T_{0}$ for both, coinciding with the NFL alloy $x=0.1$ and $p=0$ (Fig. 7). At $6.9 \mathrm{kbar}$ for $x=0.2$, the clear suppression of the low- $T$ increase of $C / T$ towards the data for $\mathrm{CeCu}_{6}$ indicates restoration of the FL (not shown), i.e., one can pressure-tune $\mathrm{CeCu}_{5.8} \mathrm{Au}_{0.2}$ all the way from antiferromagnetic order through the QCP to FL (v. Löhneysen, 1996b). Likewise, application of $p=6.0 \mathrm{kbar}$ for $x=0.1$ drives this alloy even further towards FL behavior (Fig. 7), as $C / T$ at low $T$ now falls even below the $p=0$ data of $\mathrm{CeCu}_{6}$.

We point out one peculiar feature of the $C / T$ data: Both for the concentration- and pressure-driven transitions, $C / T$ at e.g. $100 \mathrm{mK}$ continues to increase - as a function of $x$ or $p$-beyond the QCP when moving from the disordered to the ordered phase, Fig. 7. This behavior parallels that of the entropy, Fig. 8, discussed above.

The magnetization $M(T)$ measured in a field $B=$ $0.1 \mathrm{~T}$ for $x=0.1$ exhibits a cusp for $T \rightarrow 0$ which can be modeled as $\chi \approx M / B=\chi_{0}-\alpha \sqrt{T}$ between $80 \mathrm{mK}$ and $3 \mathrm{~K}$. Roughly the same $T$ dependence of $M / B$ is found upon reduction of the field to $0.01 \mathrm{~T}$ with a slightly stronger upturn towards low $T$ below $0.3 \mathrm{~K}$ (v. Löhneysen et al., 1998a). The $0.1 \mathrm{~T}$ data can be very well described also by a different functional dependence, i.e., $\chi(T)^{-1}-\chi(0)^{-1} \propto T^{\alpha}$ with $\alpha=0.8$ (Schröder et al., 1998), see below.

Fig. 9 shows $\rho(T)$ for different $\mathrm{CeCu}_{6-x} \mathrm{Au}_{x}$ alloys for current parallel to the orthorhombic $a$-axis. For $x<x_{c} \approx 0.1, \rho(T)$ increases at low $T$ as $\rho(T)=\rho_{0}+A T^{2}$ 


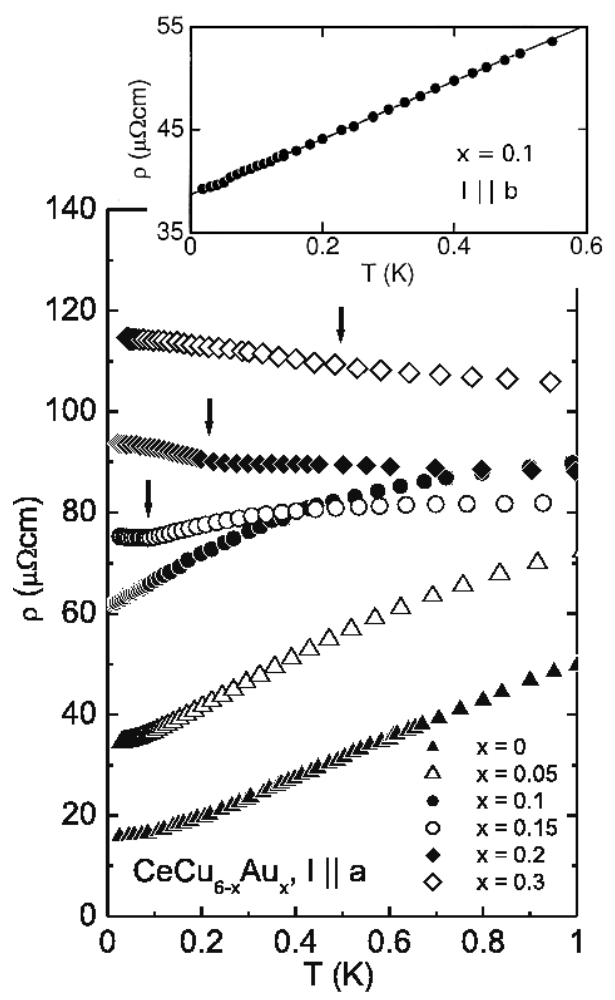

FIG. 9 Electrical resistivity $\rho$ of $\mathrm{CeCu}_{6-x} \mathrm{Au}_{x}$ vs. temperature $T$, with current applied to the $a$ direction. Arrows indicate the Néel temperature. Inset shows data for $x=0.1$ along the $b$ direction. For all directions, $\rho=\rho_{0}+A^{\prime} T$ is observed. From v. Löhneysen et al., 1998a.

which is expected for a FL with dominant quasiparticlequasiparticle scattering for $T \rightarrow 0$. This has been observed before for $\mathrm{CeCu}_{6}$ (Amato et al., 1987). For $x=0.1$ a linear $T$ dependence of $\rho$ is observed between $20 \mathrm{mK}$ and $0.6 \mathrm{~K}$ (see Fig. 9), signaling NFL behavior. The anisotropic $\rho(T)$ dependence of the magnetically ordered alloys can be qualitatively interpreted in terms of the observed magnetic order: $\rho(T)$ for all alloys except $x=1$ increases below $T_{\mathrm{N}}$ for current directions with a non-zero projection of the magnetic ordering vector $\mathbf{Q}$ determined from elastic neutron scattering (v. Löhneysen et al., 1998b). An increase of $\rho(T)$ below $T_{\mathrm{N}}$ has been observed before in other HFS, for example, in $\mathrm{CeRu}_{2-x} \mathrm{Rh}_{x} \mathrm{Si}_{2}$ as will be discussed below (Miyako et al., 1997).

The abundance of low-energy magnetic excitations as $T_{\mathrm{N}} \rightarrow 0$ has been suggested to cause the NFL behavior at the magnetic instability (v. Löhneysen et al., 1994). This is supported by the recovery of FL behavior in high magnetic fields $B$ (Finsterbusch et al., 1996; v. Löhneysen et al., 1994). A negative deviation from the $C / T \sim \ln \left(T_{0} / T\right)$ divergence is seen for all fields $B \geq 0.2 \mathrm{~T}$, with a crossover temperature roughly obeying $T_{\text {cr }} \sim B$. A similar systematic recovery of FL behavior of a quantum critical system upon application of a magnetic field has been observed in many other systems. We add that the high-field specific heat of all $\mathrm{CeCu}_{6-x} \mathrm{Au}_{x}$ alloys including $x=0.1$ can be reasonably well described (Schlager et al., 1993; v. Löhneysen et al., 1996a) within a single-ion Kondo model.

The $\ln \left(T_{0} / T\right)$ dependence of $C / T$ and the linear $T$ dependence of $\rho$ in $\mathrm{CeCu}_{6-x} \mathrm{Au}_{x}$ at the magnetic instability have constituted a major puzzle ever since they were first reported. The LGW theories for $3 \mathrm{~d}$ itinerant fermion systems predict $C / T=\gamma_{0}-\beta \sqrt{T}$ and $\Delta \rho \sim T^{3 / 2}$ for antiferromagnets $(z=2)$, while $C / T=\ln \left(T_{0} / T\right)$ and $\Delta \rho \sim T^{5 / 3}$ are expected for ferromagnets $(z=3)$, see Secs. III.C-III.F. In addition, $T_{\mathrm{N}}$ should depend on the control parameter $r_{x}=x-x_{c}$ or $r_{p}=p-p_{c}$ as $T_{\mathrm{N}} \sim|r|^{\psi}$ with $\psi=z /(d+z-2)=z /(z+1)$, Eq. (91), for $d=3$, while for $\mathrm{CeCu}_{6-x} \mathrm{Au}_{x} \psi=1$ for both $r_{x}$ and $r_{p}$. Rosch et al. (1997) showed in an analysis similar in spirit to that of Millis (1993) that 2d critical fluctuations coupled to quasiparticles with $3 \mathrm{~d}$ dynamics lead to the observed behavior $C / T \sim \ln \left(T_{0} / T\right), \Delta \rho \sim T$ and $T_{\mathrm{N}} \sim|r|$.

Let us discuss the question of $2 \mathrm{~d}$ vs. $3 \mathrm{~d}$ magnetism in $\mathrm{CeCu}_{6-x} \mathrm{Au}_{x}$. $\mathrm{CeCu}_{6-x} \mathrm{Au}_{x}$ does exhibit $3 \mathrm{~d}$ antiferromagnetic ordering, and the anisotropy of the electrical resistivity along different crystallographic directions does not exceed a factor of 2 . Therefore $\mathrm{CeCu}_{6-x} \mathrm{Au}_{x}$ looks like a $3 \mathrm{~d}$ antiferromagnetic metal. The magnetic structure of $\mathrm{CeCu}_{6-x} \mathrm{Au}_{x}(0.15 \leq x \leq 1)$ has been investigated with elastic neutron scattering (Okumura et al., 1998; v. Löhneysen et al., 1998b). An example of resolution-limited magnetic Bragg reflections is shown in Fig. 10. The magnetic ordering vector is $\mathbf{Q}=(0.6250$ 0.253 ) for $x=0.2$ and remains almost constant up to $x$ $=0.4$. For larger $x$ it jumps onto the $a^{*}$ axis, $\mathbf{Q}=(0.56$ $00)$ for $x=0.5$ and $(0.5900)$ for $x=1$.

A detailed investigation of the critical fluctuations at $x_{c}=0.1$ using inelastic neutron scattering (Stockert et al., 1998) showed that the critical fluctuations are strongly anisotropic and extend into the $a^{*} c^{*}$ plane. This is inferred from a large number of $l$ scans in the $a^{*} c^{*}$ plane, some of which are shown in Fig. 11. Hence the dynamical structure factor $S(\mathbf{q}, \hbar \omega=0.15 \mathrm{meV})$ has the form of rods (see Fig. 10). Since a quasi-1d feature in reciprocal space corresponds to quasi-2d fluctuations in real space, the 2d LGW scenario (Rosch et al., 1997) appears to be applicable. The width of $S(\mathbf{q}, \omega)$ perpendicular to the rods is roughly a factor of five smaller than along the rods. It is an issue of current debate whether this anisotropy of the correlation length is enough to qualify the fluctuations as being $2 \mathrm{~d}$. The $3 \mathrm{~d}$ ordering peaks for $x=0.2$ and 0.3 fall on the rods for $x=0.1$ which therefore can be viewed as a precursor to $3 \mathrm{~d}$ ordering (Fig. 10). Fig. 11 demonstrates the essentially similar, albeit broader $S(\mathbf{q}, \hbar \omega=$ const $)$ dependence for samples away from the critical concentration, i.e., for $x=0$ and 0.2 (v. Löhneysen et al., 2002).

The dynamic structure factor $S(\mathbf{q}=$ const, $\hbar \omega)$ of $\mathrm{CeCu}_{6-x} \mathrm{Au}_{x}$ was investigated around $\mathbf{Q}=\left(\begin{array}{ll}0.80 & 0\end{array}\right)$, i.e., on the rods (Fig. 10), by Schröder et al. (1998). They found a scaling of the dynamical susceptibility of the form

$$
\chi^{-1}(\mathbf{q}, E, T)=c^{-1}\left[f(\mathbf{q})+(-i E+a T)^{\alpha}\right]
$$



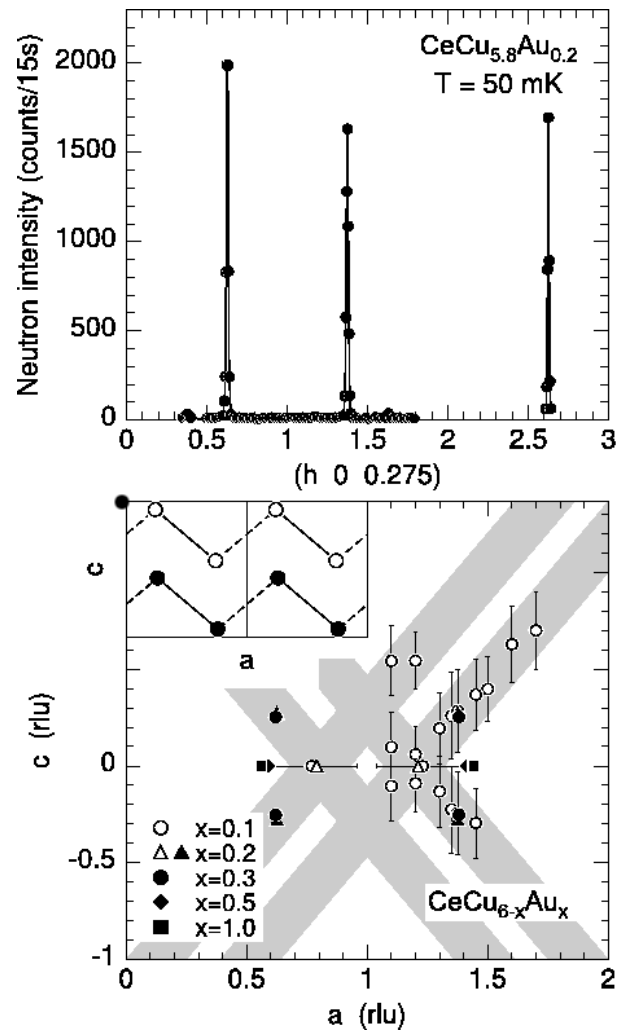

FIG. 10 Neutron scattering results for $\mathrm{CeCu}_{6-x} \mathrm{Au}_{x}$. Upper panel: Resolution-limited magnetic Bragg reflections for $\mathrm{CeCu}_{5.8} \mathrm{Au}_{0.2}$ corresponding to an incommensurate magnetic

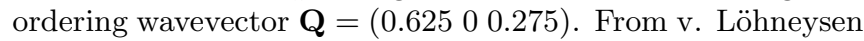
et al., 1998b. Lower panel: Position of the dynamic correlations $(x=0.1, \hbar \omega=0.1 \mathrm{meV}, T<100 \mathrm{mK})$ and magnetic Bragg peaks $(0.2 \leq x \leq 1.0)$ in the $a^{*} c^{*}$ plane in $\mathrm{CeCu}_{6-x} \mathrm{Au}_{x}$. Closed symbols for $x=0.2$ represents shortrange order peaks. The vertical and horizontal bars indicate the Lorentzian linewidths for $x=0.1$. The four shaded rods are related by the orthorhombic symmetry (we ignore the small monoclinic distortion). The inset shows a schematic projection of the $\mathrm{CeCu}_{6-x} \mathrm{Au}_{x}$ structure onto the $a c$ plane where only the Ce atoms are shown. The rods in reciprocal space correspond to planes in real space spanned by $b$ and lines in the inset. From Stockert et al., 1998.

with an anomalous scaling exponent $\alpha=0.74$ (a Lorentzian fluctuation spectrum would be described by $\alpha=1)$. This translates to

$$
\begin{aligned}
\chi^{\prime \prime}(E, T) & =T^{-\alpha} g\left(E / k_{B} T\right), \\
g(y) & =c \sin \left[\alpha \tan ^{-1}(y)\right] /\left(y^{2}+1\right)^{\alpha / 2} .
\end{aligned}
$$

Fig. 12 shows the scaling obtained by Schröder et al. (1998), which is confirmed by more recent data (Schröder et al., 2000). The exponent $\alpha \approx 0.8$ implies $z=2 / \alpha=$ 2.5. It is interesting to note that Eq. (149) implies for the static uniform susceptibility $(E=0, q=0)$

$$
\left[\chi^{\prime}(T)^{-1}-\chi^{\prime}(0)^{-1}\right]=c^{-1}(a T)^{\alpha} .
$$

Schröder et al. (1998) showed that indeed the static uniform susceptibility can be described by Eq. (152) with $\alpha=0.8$ between 0.08 and $8 \mathrm{~K}$ in agreement with $\alpha=0.74$ from neutron scattering. The fact that this quantum critical scaling persists to temperatures well above $T_{\mathrm{K}}$ appears surprising.

The two experiments by Stockert et al. (1998) and Schröder et al. (1998) are complementary in that the former focuses on the $q$ dependence and the latter on the $E$ dependence of $\chi(\mathbf{q}, E)$ of the critical fluctuations in $\mathrm{CeCu}_{6-x} \mathrm{Au}_{x}$. The data agree qualitatively in the range where they overlap. An open question is the origin of the fluctuation spectrum being effectively two-dimensional, and whether this fact may be generic for certain Ce-based materials. It is clear that the $2 \mathrm{~d}$ planes are spanned by the $b$ axis and the connection between next-nearest neighbor Ce atoms (see Fig. 10). Only more detailed investigations can establish if, perhaps, the low-dimensionality arises from a strong spatial anisotropy or frustration of the RKKY interaction or the $c-f$ hybridization.

The unusual type of scaling in the dynamical susceptibility of $\mathrm{CeCu}_{6-x} \mathrm{Au}_{x}$ at the QPT is incompatible with the LGW model - there, $E / T$ scaling as in Eq. (149) is only expected below the upper critical dimension (which is $d=2$ for the metallic antiferromagnet). The experiments therefore prompted new theoretical concepts, based on the idea that at the QCP the (lattice) Kondo effect breaks down (Sec. III.I). Specific proposals include a so-called "local" quantum critical point (Si et al., 2001, 2003), spin-charge separation (Coleman et al., 2001; Pépin, 2005), or fractionalization of the Fermi liquid (Senthil, Sachdev, and Vojta, 2003; Senthil, Vojta, and Sachdev, 2004). The local QCP scenario of Si et al. (2001) requires the existence of two-dimensional critical spin fluctuations; as mentioned above, this is not inconsistent with the experiments. Among the currently

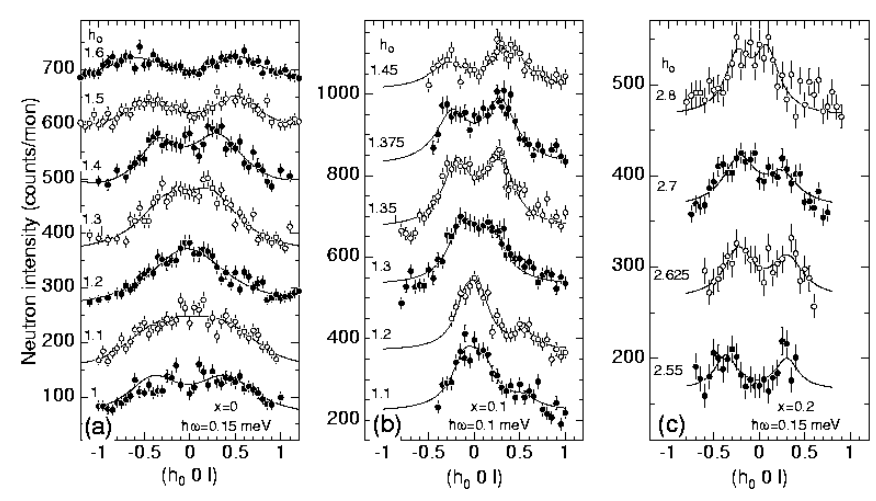

FIG. 11 Inelastic neutron scattering scans in the reciprocal $a^{*} c^{*}$ plane of $\mathrm{CeCu}_{6-x} \mathrm{Au}_{x}$ for (a) $x=0$ with an energy transfer $\hbar \omega=0.15 \mathrm{meV}$, (b) $x=0.1, \hbar \omega=0.10 \mathrm{meV}$, and (c) $x=0.2, \hbar \omega=0.15 \mathrm{meV}$. Data were taken at the triple axis spectrometers $\operatorname{IN12}(x=0)$ and $\operatorname{IN} 14(x=0.1 ; 0.2)$ at the ILL Grenoble. The individual scans are shifted by 100 counts $(x=0 ; 0.2)$ for 150 counts $(x=0.1)$ with respect to each other. Scans along $(2.8-\xi 0 l)$ for $x=0.2$ are symmetry equivalent to $(1.2 \xi 0 l)$ scans $(x=0 ; 0.1)$. From v. Löhneysen et al., 2002 . 


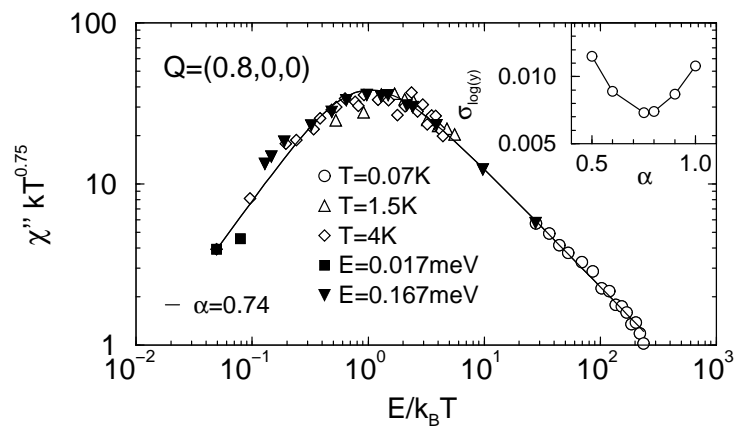

FIG. 12 Scaling plot of inelastic neutron scattering data for $\mathrm{CeCu}_{5.8} \mathrm{Au}_{0.2}$ at $q=\left(\begin{array}{lll}0.8 & 0 & 0\end{array}\right)$ vs. $E / k_{\mathrm{B}} T$. Solid line corresponds to a fit of the scaling function Eq. (150) with $\alpha=0.74$. Inset shows how the quality of the scaling collapse varies with $\alpha$. From Schröder et al., 1998.

available scenarios, only the local QCP picture contains $E / T$ scaling in the dynamical susceptibility (by virtue of the model at all wavevectors). Moreover, the fractional exponent obtained from numerical calculations of the EDMFT equations (Grempel and Si, 2003), in the experimentally relevant situation of Ising symmetry, is $\alpha \approx 0.72$, i.e., close to the experimental value of $\approx 0.74$.

As discussed in Sec. III.I, the breakdown of the Kondo effect should be connected with an additional energy scale (associated with Fermi surface fluctuations) vanishing at the QCP, apart from that of magnetic ordering. Experimentally, the situation is ambiguous. In $\mathrm{CeCu}_{6-x} \mathrm{Au}_{x}$ essentially all experimental results indicate that some scale of the order of a few Kelvin stays finite at the QCP for a doping of $x=0.1$. This can be seen from the existence of a maximum in the resistivity at $T_{\mathrm{m}} \approx 4 \mathrm{~K}$ (for current flow along the a direction) (v. Löhneysen et al., 1998a, 2002), or the temperature $T_{1 / 2}$, where the entropy reaches $0.5 R \ell n 2$ (Fig. 8). (For $\mathrm{YbRh}_{2} \mathrm{Si}_{2}$ similar conclusions on $T_{1 / 2}$ can be drawn from the specificheat data. However, for this material recent magnetostriction measurements have indicated the existence of thermodynamic low-energy scales which vanish at the QCP (Gegenwart et al., 2007).) In $\mathrm{CeCu}_{6-x} \mathrm{Au}_{x}$, the specific-heat coefficient is large even in the ordered phase: As is apparent from Fig. 7 and already noted above, $C / T$ below $100 \mathrm{mK}$ is larger for the samples just above the critical concentration than for $x_{c}$, before decreasing for concentrations approaching $x=1$ (see also Mock et al. 1994).

Despite these open questions it should be stressed that $\mathrm{CeCu}_{6-x} \mathrm{Au}_{x}$ is one of the best characterized heavyfermion systems exhibiting NFL behavior. It is rewarding that the unusual behavior of the thermodynamic and transport quantities at the QCP can be traced back in a consistent fashion to an unusual low-dimensional fluctuation spectrum.

Recent measurements of the ${ }^{63} \mathrm{Cu}$ nuclear spin-lattice relaxation time $T_{1}$ on one of the five inequivalent $\mathrm{Cu}$ sites in $\mathrm{CeCu}_{5.9} \mathrm{Au}_{0.1}$ down to $0.1 \mathrm{~K}$, employing the $6.25 \mathrm{MHz}$
${ }^{63} \mathrm{Cu}$ NQR line, revealed a non-exponential nuclear magnetization recovery which was attributed to different $\mathrm{Cu}-$ neighbor configurations in the alloy (Walstedt et al., 2003). More importantly, $T_{1}^{-1}$ was found to vary as $T^{0.75}$ at low $T$. Since $T_{1}^{-1}$ is proportional to the weighted squared average $\left\langle A(\mathbf{q})^{2}\right\rangle$ of transverse hyperfine couplings over the Brillouin zone, the "agreement" with the exponent $\alpha$ found in the $T$ and $E$ dependence of $\chi(\mathbf{q}, \omega)$ (149) is entirely accidental.

Although $\mathrm{CeCu}_{6}$ alloyed with $\mathrm{Au}$ is the most thoroughly studied system, other alloys derived from $\mathrm{CeCu}_{6}$ have been widely studied as well. Magnetic ordering in the system $\mathrm{CeCu}_{6-x} \mathrm{Ag}_{x}$ was discovered simultaneously with $\mathrm{CeCu}_{6-x} \mathrm{Au}_{x}$ (Fraunberger et al., 1989; Gangopadhyay et al., 1988; Germann et al., 1988). $\mathrm{CeCu}_{6-x} \mathrm{Ag}_{x}$ displays critical behavior around $x_{c}=0.2$, with thermodynamic properties being very similar to $\mathrm{CeCu}_{5.9} \mathrm{Au}_{0.1}$, e.g., a logarithmic divergence of $C / T$. Interestingly, thermal expansion measurements have uncovered that the critical behavior is incompatible with both the $3 \mathrm{~d}$ and 2d LGW model: the Grüneisen parameter $\Gamma$, Eq. (70), is expected to diverge in the quantum critical regime for $T \rightarrow 0$ as $\Gamma \sim T^{-1 /(z \nu)}$ with $z \nu=1$. However, the experiment shows a much weaker (logarithmic) divergence of $\Gamma$ (Küchler et al., 2004).

For field-tuned transitions the behavior appears to be different: NFL behavior in a polycrystalline $\mathrm{CeCu}_{4.8} \mathrm{Ag}_{1.2}$ alloy, subjected to a magnetic field, was reported, i.e., approximately $C / T \sim \ln \left(T_{0} / T\right)$ between 0.35 and $2.5 \mathrm{~K}$ (Heuser et al., 1998a). However, in the light of the strong magnetic anisotropy of this system, with $B$ affecting crystallites of different orientations quite differently, this result should be viewed with caution. Subsequently, Scheidt et al. (1998) reported specific-heat data down to $0.07 \mathrm{~K}$ on a $\mathrm{CeCu}_{5.2} \mathrm{Ag}_{0.8}$ single crystal with $T_{\mathrm{N}}=0.7 \mathrm{~K}$. For a critical magnetic field $B_{c}=2.3 \mathrm{~T}$ applied along the easy direction, $C / T$ varies logarithmically from $\sim 1.5 \mathrm{~K}$ down to $0.2 \mathrm{~K}$ and then levels off. Furthermore, the resistivity $\rho(T)$ of $\mathrm{CeCu}_{5.2} \mathrm{Ag}_{0.8}$ can be described by a $T^{1.5}$ dependence at $B_{c}$. The authors interpret the data within the LGW scenario of Moriya and Takimoto (1995), Sec. III.E, with $d=3$ and $z=2$.

A detailed comparison of pressure-tuned and fieldtuned $\mathrm{QCP}$ on the same system $\mathrm{CeCu}_{5.8} \mathrm{Au}_{0.2}$ (v. Löhneysen et al., 2001) demonstrated that field, as opposed to pressure, drives the system to a 3d LGW quantum critical point. The data $\rho(T)$ and $C / T$ for $\mathrm{CeCu}_{5.2} \mathrm{Ag}_{0.8}$ for $B_{c}$ look quite similar to those for $\mathrm{CeCu}_{5.8} \mathrm{Au}_{0.2}$ for $B=0.3$ or $0.5 \mathrm{~T}$, i.e., in the region of $B_{c} \approx 0.4 \mathrm{~T}$ as determined from neutron scattering. This difference to concentration and - very likely - also to pressure tuning was recently directly confirmed by inelastic neutron scattering where the observed $\omega / T^{1.5}$ scaling corresponds to the LGW scenario (Stockert et al., 2006b). It is open whether the apparent similarity to the spin-fluctuation theory represents cross-over phenomena or the approach to a new QCP.

A few experiments have been performed on fur- 
ther $\mathrm{CeCu}_{6}$-derived alloys. For $\mathrm{CeCu}_{6-x} \mathrm{Pd}_{x}$ and $\mathrm{CeCu}_{6-x} \mathrm{Pt}_{x}$ the NFL behavior was found in the specific heat at the critical concentration for the onset of magnetic order $\left(x_{c} \approx 0.05\right.$ for $\mathrm{Pd}$ and $\approx 0.1$ for $\left.\mathrm{Pt}\right)$, while for $\mathrm{CeCu}_{6-x} \mathrm{Ni}_{x}$ a non-magnetic ground state is stabilized for small $x$ (Sieck et al., 1996). Notably, the $C / T$ data for the $\mathrm{Pd}$ and $\mathrm{Pt}$ alloys at their respective $x_{c}$ agree well with those for $\mathrm{CeCu}_{6-x} \mathrm{Au}_{x}$ at the quantum critical point, suggesting universality for all $\mathrm{QPT}$ in $\mathrm{CeCu}_{6}$ alloys in zero magnetic field. Note, however, that recent data on a $\mathrm{CeCu}_{6-x} \mathrm{Ag}_{x}$ polycrystal with $x=0.2$, believed to be close to the quantum-critical concentration, exhibit a somewhat steeper slope of $C / T$ vs. $\ln \left(T_{0} / T\right)$, with, however, a gentle leveling-off towards low $T$, leading in effect to a $C / T$ larger by $20 \%$ at $0.1 \mathrm{~K}$ compared to $\mathrm{CeCu}_{5.9} \mathrm{Au}_{0.1}$ (Küchler et al., 2004).

\section{2. $\mathrm{Ce}_{1-x} \mathrm{La}_{x} \mathrm{Ru}_{2} \mathrm{Si}_{2}$ and $\mathrm{Ce}\left(\mathrm{Rh}_{1-x} \mathrm{Ru}_{x}\right)_{2} \mathrm{Si}_{2}$}

$\mathrm{CeRu}_{2} \mathrm{Si}_{2}$ is, like $\mathrm{CeCu}_{6}$, a canonical heavy-fermion system without apparent magnetic order. It crystallizes in the tetragonal $\mathrm{I} / 4 \mathrm{mmm}$ structure. The ground state doublet of $\mathrm{Ce}^{3+}$ in $\mathrm{CeRu}_{2} \mathrm{Si}_{2}$ exhibits Ising character (Regnault et al., 1988). Static magnetism with very small ordered moment was detected by muon-spin-rotation experiments (Amato et al., 1994). The magnetic order develops below about $2 \mathrm{~K}$ and the ordered moment reaches a value of $\sim 10^{-3} \mu_{B} /$ Ce-atom at low temperatures $(T<$ $0.1 \mathrm{~K})$. The Kondo coupling between Ce $4 f$ electrons and conduction electrons is stronger than in $\mathrm{CeCu}_{6}$, this is also suggested by the rather low (for a heavy-fermion system) $\gamma$ value of $0.36 \mathrm{~J} / \mathrm{molK}^{2}$ (Fisher et al., 1991). From specific-heat data $T_{\mathrm{K}}$ is found to be $20 \mathrm{~K}$. Magnetic intersite correlations are also much stronger in $\mathrm{CeRu}_{2} \mathrm{Si}_{2}$ than in $\mathrm{CeCu}_{6}$. This has been shown by inelastic neutron scattering where $q$-dependent correlations are peaked at $k_{1}=\left(\begin{array}{lll}0.3 & 0 & 0\end{array}\right)$ and $k_{2}=\left(\begin{array}{lll}0.3 & 0.3 & 0\end{array}\right)$ (Rossat-Mignod et al., 1988). In a recent inelastic neutron scattering study, the spin fluctuations were interpreted within the LGW model (Kadowaki, Sato, and Kawarazaki, 2004).

A metamagnetic transition occurs in a field of $B_{M} \approx$ $8 \mathrm{~T}$ (Haen et al., 1987; Holtmeier et al., 1995), which manifests itself as a steep jump in the magnetization curve $M(B)$. The corresponding maximum in $d M / d B$ at $B_{M}=8 \mathrm{~T}$ is much more pronounced and much sharper than in $\mathrm{CeCu}_{6}$ at $B_{M}=2 \mathrm{~T}$. It is interesting to note in the present context that all the way through the metamagnetic transition, $\mathrm{CeRu}_{2} \mathrm{Si}_{2}$ exhibits well-defined $\mathrm{FL}$ behavior as evidenced by the prevalence of de Haas-van Alphen ( $\mathrm{dHvA})$ oscillations very close to the transition (Tautz et al., 1995). Some of the branches could be detected to within $0.005 \mathrm{~T}$ of $B_{M}=7.8 \mathrm{~T}$. However, the branch of the heavy quasiparticles is strongly affected by the metamagnetic transition: its effective mass changes from $m^{*}=12 m_{0}$ at $6 \mathrm{~T}$ to $4 m_{0}$ at $12.5 \mathrm{~T}$.

Alloying $\mathrm{CeRu}_{2} \mathrm{Si}_{2}$ on the Ce site, i.e., diluting with non-magnetic La, leads to long-range antiferromagnetic

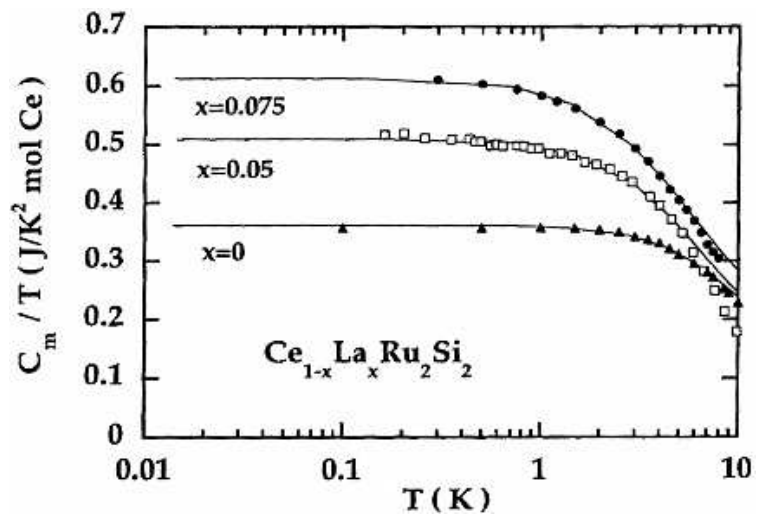

FIG. 13 Magnetic contribution to the specific heat divided by $T, C_{m} / T$, vs. temperature $T$ (logarithmic scale) for $\mathrm{Ce}_{1-x} \mathrm{La}_{x} \mathrm{Ru}_{2} \mathrm{Si}_{2}$ for $x=0,0.05$ and 0.075 . Solid lines are fits to the LGW model. From Kambe et al., 1996.

order in $\mathrm{Ce}_{1-x} \mathrm{La}_{x} \mathrm{Ru}_{2} \mathrm{Si}_{2}$. This might at first sight be even more surprising than the observation of antiferromagnetism induced by $\mathrm{Au}$-doping of $\mathrm{CeCu}_{6}$. It may, however, be simply attributed to a lattice expansion caused by replacing Ce by La (the famous lanthanide contraction backwards), leading to a strong reduction of $T_{\mathrm{K}}$ which outweighs the reduction of the number of $\mathrm{Ce} 4 f$ moments for small $x$. This is nicely supported by measurements on $\mathrm{Ce}_{1-x}\left(\mathrm{La}_{0.63} \mathrm{Y}_{0.37}\right)_{x} \mathrm{Ru}_{2} \mathrm{Si}_{2}$ which is isochoric to $\mathrm{CeRu}_{2} \mathrm{Si}_{2}$ and does not show magnetic order (Sakakibara et al., 1993).

The long-range antiferromagnetic order for $x \geq 0.1$ manifests itself by maxima in specific heat and magnetic susceptibility (Fisher et al., 1991) and in an increase of the electrical resistivity below the ordering temperature (Djerbi et al., 1988). Applying hydrostatic pressure decreases $T_{\mathrm{N}}$ as shown for $x=0.2$ where $T_{\mathrm{N}} \rightarrow 0$ for $p_{c} \approx$ $7 \mathrm{kbar}$. At the same time, the ordered moment as derived from elastic neutron scattering vanishes (Regnault et al., 1990). However, the pressure dependence $T_{\mathrm{N}} \sim\left|p-p_{c}\right|^{\psi}$ is clearly sub-linear. This matches the sub-linear $T_{\mathrm{N}}(x)$ dependence where $T_{\mathrm{N}}$ vanishes for a critical concentration $x_{c} \approx 0.075$ (Quezel et al., 1988).

Upon approaching $x_{c}=0.075$ where $T_{\mathrm{N}} \rightarrow 0$, the specific-heat coefficient increases towards low $T$ (Flouquet et al., 1995). Kambe et al. (1996) performed additional experiments for $x=0.075$ which clearly show the strong enhancement of $C / T$. They describe the data consistently in terms of the LGW model as applied to heavy-fermion systems by Moriya and Takimoto (1995). In contrast to $\mathrm{CeCu}_{6-x} \mathrm{Au}_{x}$, the data can be quantitatively interpreted within the latter model for a $3 \mathrm{~d}$ antiferromagnet, i.e., the specific-heat coefficient $\gamma$ crosses over from a logarithmic $T$ dependence in an intermediate $T$ range to $C / T=\gamma_{0}-\beta \sqrt{T}$ for $T \rightarrow 0$ (Fig. 13), which is the asymptotic behavior within the LGW model, Sec. III.D. Likewise, the electrical resistivity at the critical concentration shows the LGW dependence for a disordered antiferromagnet, $\Delta \rho \sim T^{3 / 2}$ (Kambe et al., 1996). 
Inelastic neutron-scattering experiments on $\mathrm{Ce}_{0.925} \mathrm{La}_{0.075} \mathrm{Ru}_{2} \mathrm{Si}_{2}$ showed three-dimensional correlations for this system (Knafo et al., 2004; Raymond

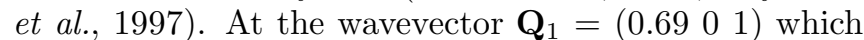
corresponds to antiferromagnetic order, an approximate scaling of the form

$$
\chi^{\prime \prime}(E, T)=T^{-1} g\left(E /\left(k_{\mathrm{B}} T\right)^{0.8}\right)
$$

with $g(y)=y /(1+y)^{2}$ was observed, however, only for $T \geq 5 \mathrm{~K}$. As discussed by Knafo et al. (2004), this apparent scaling is probably related to a crossover regime, see Sec. I.C, as the Kondo temperature in this system is about $17 \mathrm{~K}$. Furthermore, the linewidth of the dynamical fluctuations does not vanish for $T \rightarrow 0$ as it should for a quantum critical point. This may indicate that $x=0.075$ is a little off the QCP. Nevertheless, the linewidth is reduced by a factor of 5 compared to the pure compound while the correlation length for $T \rightarrow 0$ is only 1.5 times larger than for pure $\mathrm{CeRu}_{2} \mathrm{Si}_{2}$. The dynamic susceptibility $\chi(\mathbf{q}, \omega)$ is interpreted in terms of the RPA, which yields an overall satisfactory agreement between the parameters of the LGW model as derived from inelastic neutron scattering and those derived from the specific heat (Raymond et al., 1997). As a final point we mention that in addition to the inelastic signal, Raymond et al. (1997) observed below $1.8 \mathrm{~K}$ a very small elastic signal at $\mathbf{Q}=\left(\begin{array}{lll}0.6910 & 10\end{array}\right)$ for $x=0.075$. This is surprising because this sample is believed to be just below the critical concentration $x_{c}=0.08$ for the onset of magnetic order. The linewidth is only slightly larger than the instrumental resolution, and the ordered moment is $\sim 0.02 \mu_{B}$ at the lowest temperature which may be due to concentration fluctuations. The evolution of spin dynamics was also studied for an ordered sample with $x=0.13$ around the critical pressure $p_{c}=2.6 \mathrm{kbar}$ (Raymond et al., 2001).

To summarize, $\mathrm{Ce}_{1-x} \mathrm{La}_{x} \mathrm{Ru}_{2} \mathrm{Si}_{2}$ appears to be a system with a QCP exhibiting 3d critical fluctuations. Hence the LGW model describes the thermodynamic transport and neutron-scattering data rather well.

$\mathrm{CeRu}_{2} \mathrm{Ge}_{2}$ exhibits magnetic order, due to its larger $\mathrm{Ce}-\mathrm{Ce}$ separation as compared to $\mathrm{CeRu}_{2} \mathrm{Si}_{2}$. Unlike many other Ce compounds, it orders ferromagnetically below $T_{\mathrm{C}}=8 \mathrm{~K}$ (Böhm et al., 1988; Fontes et al., 1996). Upon applying pressure, the ferromagnetism is quickly suppressed, and a rather complex magnetic phase diagram with several phases evolves. By ac susceptibility measurements under pressure, and in analogy with doping studies of $\mathrm{CeRu}_{2}\left(\mathrm{Ge}_{1-x} \mathrm{Si}_{x}\right)_{2}$ where neutron scattering has been done, these phases are identified as antiferromagnetic. Magnetic order in $\mathrm{CeRu}_{2} \mathrm{Ge}_{2}$ disappears at $p_{c} \approx 6.5 \mathrm{GPa}$; at $p_{c} \mathrm{NFL}$ behavior is observed in the electrical resistivity, $\rho=\rho_{0}+A^{\prime} T$ (Süllow et al., 1999; Wilhelm et al., 1999). The system provides a nice example of the equivalence between hydrostatic pressure and chemical pressure exerted by smaller Si atoms.

$\mathrm{CeRh}_{2} \mathrm{Si}_{2}$, which is isostructural to $\mathrm{CeRu}_{2} \mathrm{Si}_{2}$, is a local-moment antiferromagnet $\left(T_{\mathrm{N}}=36 \mathrm{~K}\right)$ with a relatively large ordered moment of $\mu=1.5 \mu_{B} /$ Ce-atom
(Quezel et al., 1984). $T_{\mathrm{N}}$ can be driven to zero by hydrostatic pressure of $p_{c}=9 \mathrm{kbar}$ (Thompson, Parks, and Borges, 1986). $T_{\mathrm{N}}(p)$ varies slowly first and then precipitously drops to zero upon approaching $p_{c}$ (Movshovich et $a l ., 1996)$. This may be suggestive of a first-order transition near $T=0$, perhaps even stronger than the one observed in MnSi (Pfleiderer et al., 1997), Sec. IV.B.2. Clearly, a first-order transition would cut off quantum fluctuations. Consequently, anomalies in the specific heat associated with NFL behavior were not observed in $\mathrm{CeRh}_{2} \mathrm{Si}_{2}$ around $p_{c}$ (Graf et al., 1997). In addition, the resistivity above $p_{c}$ exhibits a FL-like $T^{2}$ behavior (Movshovich et al., 1996). It is interesting to note that in the vicinity of $p_{c}$ and beyond, $\mathrm{CeRh}_{2} \mathrm{Si}_{2}$ becomes superconducting with $T_{c}$ between 0.2 and $0.4 \mathrm{~K}$ (Movshovich et al., 1996), see also Sec. IV.C.

The magnetic phase diagram of $\mathrm{Ce}\left(\mathrm{Ru}_{1-x} \mathrm{Rh}_{x}\right)_{2} \mathrm{Si}_{2}$ has been studied by several groups (Calemczuk et al., 1990; Kawarazaki et al., 1995; Lloret et al., 1987) with a number of techniques, including neutron scattering. The data reveal a complex $T_{\mathrm{N}}(x)$ phase diagram with two different antiferromagnetic phases, one between $x \approx 0.05$ and 0.27 , with a maximum $T_{\mathrm{N}}$ of $\sim 5 \mathrm{~K}$, and the other phase emanating from $\mathrm{CeRh}_{2} \mathrm{Si}_{2}(x=1)$, with $T_{\mathrm{N}}$ decreasing steeply to $\sim 13 \mathrm{~K}$ for $x=0.95$ and then exhibiting a plateau at $T_{\mathrm{N}}=12 \mathrm{~K}$ until $x=0.6$, when it drops again and reaches $T_{\mathrm{N}}=0$ around $x_{c} \approx 0.5$. The magnetic structure in both concentration ranges has been determined by elastic neutron scattering. For the $\mathrm{CeRh}_{2} \mathrm{Si}_{2}$ derived phase, a wavevector $\mathbf{Q}=\left(\frac{1}{2} \frac{1}{2} 0\right)$ corresponding to an antiferromagnetic coupling within ( $\left.\begin{array}{lll}0 & 0 & 1\end{array}\right)$ planes (Quezel et al., 1984) was reported to exist down $x=0.6$ (Lloret et al., 1987), with ordered moments $1.5 \mu_{B} / \mathrm{Ce}-$ atom for $x=1$ and $0.65 \mu_{B} /$ Ce-atom for $x=0.65$. This structure was essentially confirmed by Kawarazaki et al. (1995), although these authors observed for $x=1$ additional reflections corresponding to $\mathbf{Q}^{\prime}=\left(\begin{array}{lll}\frac{1}{2} & \frac{1}{2} & \frac{1}{2}\end{array}\right)$ which already had been seen by Grier et al. (1984). It is not clear whether this behavior arises from a homogeneous single phase or a domain structure.

A large effort has been devoted to analyze the low- $x$ magnetic phase centered around $x=0.15$. A sinusoidal spin-density wave with the moments ordered along the propagation axis has been inferred (Kawarazaki et al., 1995; Miyako et al., 1996) which shifts from incommensurate to commensurate: $\mathbf{Q}=\left(\begin{array}{lll}0 & 0 & 0.42\end{array}\right),\left(\begin{array}{lll}0 & 0 & 0.45\end{array}\right)$ and $\left(\begin{array}{lll}0 & 0 & 0.5\end{array}\right)$ for $x=0.15,0.2$, and 0.25 , respectively. This magnetic structure is quite different from that of La-diluted $\mathrm{CeRu}_{2} \mathrm{Si}_{2}$ discussed above. Recent inelastic neutron scattering experiments for $x=0.03$ revealed a $T^{3 / 2}$ dependence of the linewidth at the antiferromagnetic wavevector $\mathbf{Q}=\left(\begin{array}{lll}0 & 0 & 0.35\end{array}\right)$, compatible with the LGW scenario (Kadowaki et al., 2006).

It should be mentioned that $\rho(T)$ increases strongly below $T_{\mathrm{N}}$ which is interpreted as arising from a spin-density wave (Miyako et al., 1997; Murayama et al., 1997). A detailed scenario for Fermi-surface nesting has been developed for $x=0.15$ (Miyako et al., 1996). Deviations 
from FL behavior have been observed in the region where $T_{\mathrm{N}} \approx 0$ for $x=0.5$ (Graf et al., 1997) and $x=0.4$ (Taniguchi et al., 1998) in both specific heat and magnetic susceptibility. While initial data for $x=0.4$ show $C / T \sim \ln \left(T_{0} / T\right)$ between $10 \mathrm{~K}$ and $0.15 \mathrm{~K}$, data taken by the same group with higher precision indicate a slight leveling off below $1 \mathrm{~K}$ ( $\mathrm{Gu}$ et al., 2002). $C / T$ for $x=0.5$ levels off more strongly below $\sim 1 \mathrm{~K}$. At least part of the data can be described by a Kondo disorder model with a distribution of $T_{\mathrm{K}}$ (Graf et al., 1997).

Let us point out that the $T_{\mathrm{N}}(x)$ functional dependence of $\mathrm{Ce}\left(\mathrm{Ru}_{1-x} \mathrm{Rh}_{x}\right)_{2} \mathrm{Si}_{2}$ for $x>0.5$ does not at all resemble the $T_{\mathrm{N}}(p)$ dependence of $\mathrm{CeRh}_{2} \mathrm{Si}_{2}$. This might serve as a warning in taking "chemical pressure" effects too literally and reducing changes brought about by alloying to simple volume changes. Even for $\mathrm{CeCu}_{6-x} \mathrm{Au}_{x}$ where the functional dependence of $T_{\mathrm{N}}(p)$ and $T_{\mathrm{N}}(x)$ are the same (both with an exponent $\psi=1$ ) as discussed above, and where isoelectronic substituents are used ( $\mathrm{Au}$ vs. $\mathrm{Cu}$ ) as opposed to the present case (Ru vs. Rh), the calculated change of $T_{\mathrm{N}}$ with volume from $T_{\mathrm{N}}(p)$ via the compressibility does not correspond to the change calculated from $T_{\mathrm{N}}(x)$ with the x-ray determined volume $V(x)$ (Germann and v. Löhneysen, 1989; Pietrus et al., 1995). The comparison with the $\mathrm{CeRu}_{2}\left(\mathrm{Si}_{1-x} \mathrm{Ge}_{x}\right)_{2}$ system above might suggest that the chemical pressure exerted by the polyvalent metal is more akin to hydrostatic pressure than that of transition metals with partly covalent bonding.

\section{3. $\mathrm{CeCu}_{2} \mathrm{Si}_{2}$ and $\mathrm{CeNi}_{2} \mathrm{Ge}_{2}$}

$\mathrm{CeCu}_{2} \mathrm{Si}_{2}$ was the first heavy-fermion superconductor, discovered nearly 30 years ago by Steglich et al. (1979). Like the $\mathrm{CeRu}_{2} \mathrm{Si}_{2}$ family, it exhibits the tetragonal $\mathrm{I} / 4 \mathrm{mmm}$ structure. This is not the place to review the tremendous amount of work that has been done ever since on this compound, revealing a very complex ternary phase diagram in the vicinity of the 1:2:2 stoichiometric composition (Steglich et al., 1996, 1997). In a small region around this point, three different types of crystals exist with different ground states: A (magnetically ordered ground state with superconducting minority phase), S (superconducting ground state), and AS (both states almost degenerate). Which type of crystal is prepared depends on the stoichiometry, on the preparation method (polycrystals versus single crystals), and heat treatment (Steglich et al., 1996). Unfortunately, the different types could up to now not be traced to different crystallographic and/or defect-induced properties. Recently, the type of magnetic order in A-type crystals was identified by neutron diffraction (Stockert et al., 2004). The observed incommensurate magnetic order is compatible with a spin-density wave, with the ordering wavevector determined by the band structure of heavy fermions as suggested by renormalized bandstructure calculations. These findings support the early suggestion that the $\mathrm{A} / \mathrm{S}$ transition can be viewed as a
QCP (Steglich et al., 1996, 1997), where a variety of NFL features compatible with the 3D LGW scenario are observed.

The A phase in a polycrystalline sample can be suppressed by a moderate pressure $\sim 2$ kbar (Gegenwart et al., 1998) where the resistivity exhibits $\Delta \rho \sim T^{3 / 2}$. At $6.7 \mathrm{kbar}$ the specific heat can be consistently interpreted within the LGW theory for an antiferromagnet. (Specific-heat data at $2 \mathrm{kbar}$ were not reported.) However, the zero-pressure starting point does not seem to correspond to a FL: while $\Delta \rho \sim T^{2}$ above the temperature $T_{A}=0.75 \mathrm{~K}$ of the A-phase transition, $\gamma=C / T$ is strongly $T$-dependent. Another puzzle results when an S-type single crystal lacking the A-phase signature is investigated (under zero pressure). In order to suppress superconductivity, a moderate field has to be applied. At $T>0.2 \mathrm{~K}$ and for $0.2 \mathrm{~T} \leq B<6 \mathrm{~T}, \Delta \rho$ varies as $T^{3 / 2}$ and $\gamma$ almost as $\gamma_{0}-\beta \sqrt{T}$ suggesting the proximity of the QCP, and corroborating the high-pressure data of the A-phase crystal discussed above. However, when cooling below $0.2 \mathrm{~K}, \gamma$ shows a very steep upturn towards low $T$ that cannot be ascribed to nuclear Zeeman contributions. In the same $T$ range, $\Delta \rho \sim T^{3 / 2}$ is still observed. The latter is in keeping with spin-fluctuation theory for a rather disordered system $\left(\rho_{0} \approx 30 \mu \Omega \mathrm{cm}\right)$ as pointed out by Rosch $(1999,2000)$, cf. Sec. III.F. For a disordered system at the QCP, a recovery of FL behavior $\Delta \rho \sim T^{2}$ towards $T \rightarrow 0$ is not expected (Rosch, 1999). Gegenwart et al. (1998) suggest that the "disparate" behavior of transport and specific heat near the QCP in $\mathrm{CeCu}_{2} \mathrm{Si}_{2}$ is due to a "break up" of the heavy quasiparticles.

The compound $\mathrm{CeCu}_{2} \mathrm{Ge}_{2}$, isostructural to $\mathrm{CeCu}_{2} \mathrm{Si}_{2}$, orders antiferromagnetically with $T_{\mathrm{N}}=4.15 \mathrm{~K}$ (de Boer et al., 1987) which can be attributed to the larger volume and weaker $c-f$ hybridization, in keeping with the Doniach model. Upon application of a rather large pressure, superconductivity occurs for $p \approx 70-80 \mathrm{kbar}$ with $T_{c} \approx 0.6 \mathrm{~K}$ (Jaccard, Behnia, and Sierro, 1992). $T_{c}$ remains independent of pressure up to $120 \mathrm{kbar}$, reaches a maximum $T_{c}=2 \mathrm{~K}$ at $160 \mathrm{kbar}$ and drops again (Vargoz and Jaccard, 1998). The magnetic transition temperature as determined from a kink of $\rho(T)$ decreases only slightly with increasing pressure and appears to saturate around $2 \mathrm{~K}$ at $94 \mathrm{kbar}$; beyond this pressure a signature in $\rho(T)$ can no longer be observed (Jaccard et al., 1999). The appearance of superconductivity near the pressure where magnetic order is suppressed is very interesting. As in $\mathrm{CeCu}_{2} \mathrm{Si}_{2}$ (Bruls et al., 1994; Stockert et al., 2006a), superconductivity and magnetic order (A phase) appear to be mutually exclusive. Because of the possible precipitous drop of $T_{\mathrm{N}}(p)$, this transition might be of first order, thus suppressing quantum fluctuations and the concomitant NFL behavior expected only for a continuous magnetic-non-magnetic transition. It was already pointed out by Jaccard et al. (1999) that quantum critical behavior is not observed: the resistivity exponent $\alpha$ in $\Delta \rho \sim T^{\alpha}$ has almost exactly the FL value 2 at the critical pressure. 
$\mathrm{CeNi}_{2} \mathrm{Ge}_{2}$ is a stoichiometric HFS where pronounced NFL features are observed in thermodynamic properties (Gegenwart et al., 1999; Grosche et al., 2000; Küchler et al., 2003): The resistivity exponent $\alpha, \Delta \rho \sim T^{\alpha}$, varies between 1.2 and 1.5 below $4 \mathrm{~K}$ dependent on the residual resistivity $\rho_{0}$. This variation of $\alpha\left(\rho_{0}\right)$ may be understood in terms of the competition between magnetic scattering and impurity scattering (Rosch, 2000), see Sec. III.F. While early specific-heat data between $0.35 \mathrm{~K}$ and $5 \mathrm{~K}$ were found to follow $C / T \propto-\ell n T$ (Gegenwart et al., 1999), later measurements by Küchler et al. (2003) could be described by $C / T=\gamma_{0}-\beta \sqrt{T}$ as expected for the LGW scenario, when corrected for an (unexplained) steep low- $T$ upturn $C \sim T^{-2}$. (For a discussion of the sample dependence of the specific heat of this material, see Küchler et al. 2003.) The divergence of the Grüneisen parameter $\Gamma \sim T^{-1}$ for $T \rightarrow 0$ is also in line with this model (Küchler et al., 2003). It should be mentioned that in very pure $\mathrm{CeNi}_{2} \mathrm{Ge}_{2}$ samples traces of superconductivity (Gegenwart et al., 1999), or even a complete resistive transition to superconductivity (Grosche et al., 2000) were found. Thus, $\mathrm{CeNi}_{2} \mathrm{Ge}_{2}$ appears to be one of the few systems following the predictions of the LGW model for $3 \mathrm{~d}$ antiferromagnets.

\section{4. $\mathrm{Ce} T \ln _{5}(T=\mathrm{Co}, \mathrm{Rh}, \mathrm{Ir})$}

A few years ago, members of the series $\mathrm{Ce} T \operatorname{In}_{5}$ ( $T$ $=\mathrm{Ir}, \mathrm{Rh}, \mathrm{Co}$ ) were discovered as HFS with interesting ground states such as superconductivity at ambient pressure in $\mathrm{CeCoIn}_{5}$ with $T_{c}=2.3 \mathrm{~K}$ (Petrovic et al., 2001b) as well as antiferromagnetic order in $\mathrm{CeRhIn}_{5}$ with $T_{\mathrm{N}}=$ $3.8 \mathrm{~K}$ that under pressure gives way to superconductivity (Hegger et al., 2000). The crystal structure of these compounds is derived from cubic $\mathrm{CeIn}_{3}$ intercalated with $T \mathrm{In}_{2}$ layers along the $\left(\begin{array}{lll}0 & 0 & 1\end{array}\right)$ direction. This gives rise to a rather pronounced two-dimensionality in various physical properties. Alloys across this isoelectronic series display a rich variety of interplay between superconductivity and magnetic order, as can be seen in Fig. 28 below (Pagliuso et al., 2002). We will just mention a few findings about quantum criticality in these systems, focusing on the stoichiometric parent compounds. Space limitations force us to neglect the many interesting studies on alloy series, e.g., by Jeffries et al. (2005).

Although $\mathrm{CeCoIn}_{5}$ does not order magnetically, intersite magnetic correlations seem to be rather strong: transport and thermodynamic data on La-diluted $\mathrm{CeCoIn}_{5}$ were interpreted in terms of a single-ion $T_{\mathrm{K}}=$ $1.7 \mathrm{~K}$ and $T_{\text {coh }}=45 \mathrm{~K} \gg T_{\mathrm{K}}$ (Nakatsuji et al., 2002). Pronounced NFL features are observed in $\mathrm{CeCoIn}_{5}$ above the upper critical field $B_{c 2}=4.95 \mathrm{~T}$ when superconductivity is suppressed (Bianchi et al., 2003b). Fig. 14 shows $C / T$ vs. $\ln T$ for $5 \mathrm{~T} \leq B \leq 9 \mathrm{~T}$. The data can be reasonably well described by the LGW model where the parameter $y_{0}$ indicates the distance from the QCP. ( $y_{0}$ is equivalent to $r$ of Sec. III.)

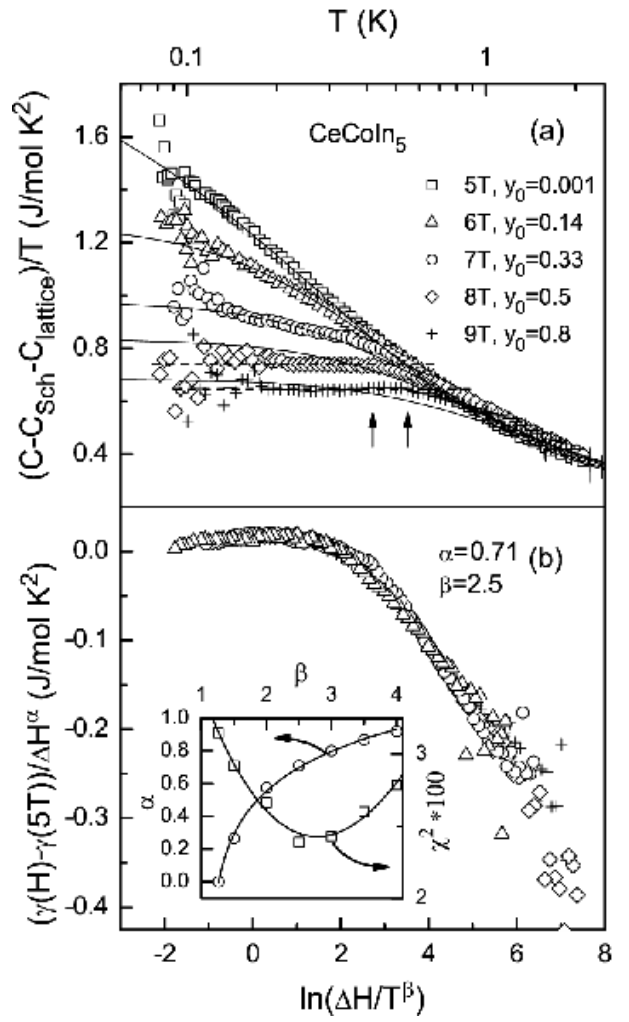

FIG. 14 Magnetic specific-heat data of $\mathrm{CeCoIn}_{5}$. (a) $\gamma(T)=$ $C_{m}(T) / T$ in magnetic fields $B=\mu_{0} H \|$ [001]. Dashed lines for 8 and $9 \mathrm{~T}$ emphasize the FL behavior with constant $\gamma$. Left(right) arrows indicate the crossover to FL behavior for $8 \mathrm{~T}(9 \mathrm{~T})$. Solid lines are fits to the LGW spin-fluctuation model for each field, with the corresponding values of $y_{0}$ indicating the distance from the critical field $B_{c}$. (b) Scaling analysis of the data in (a) for $\alpha=0.71$ and $\beta=2.5$. Inset: Plots of $\alpha(\beta)$ which minimize $\chi^{2}$ for a given $\beta$, and $\chi^{2}$ for these $\alpha$ and $\beta$. From Bianchi et al., 2003b.

At the same time, the data are shown to exhibit scaling in the form of $\gamma(B, T)-\gamma\left(B_{c}, T\right) \approx(\Delta B)^{\alpha} f\left(\Delta B / T^{\beta}\right)$ where $\Delta B=B-B_{c}$ and $B_{c} \approx B_{c 2}$ is the (quantum-) critical field. Best scaling collapse is obtained for $\alpha=$ 0.71 and $\beta=2.5$. Note, however, that a value of $\alpha \neq 0$ is not consistent with the expected recovery of FL behavior for $T \rightarrow 0$ and $B>B_{c}$, which seems to be a more likely interpretation of the experiment. Scaling with $\alpha=0$ has been previously obtained for $\mathrm{U}_{0.2} \mathrm{Y}_{0.8} \mathrm{Pd}_{3}$ with $\beta=1.3$ (Andraka and Tsvelik, 1991), for $\mathrm{YbRh}_{2} \mathrm{Si}_{2}$ with $\beta=1.05$ (Trovarelli et al., 2000), for $\mathrm{CeCu}_{x} \mathrm{Ag}_{x}$ with exponents $\beta=0.85,1.35,1.6,1.7$ for $x=0.09,0.48,0.8,1.2$ (Heuser et al., 1998a,b). At least in $\mathrm{YbRh}_{2} \mathrm{Si}_{2}$ and $\mathrm{CeCu}_{5.8} \mathrm{Ag}_{0.2}$ the scaling does not hold at the lowest $T$. In view of the different values of $\alpha$ and $\beta$, the physical significance of the scaling is not clear.

The electrical resistivity $\rho(T)$ was shown by Bianchi et al. (2003b) to follow the LGW model as discussed by Moriya and Takimoto (1995), Sec. III.E, with a set of $y_{0}$ parameters consistent with the specific heat. The small value $y_{0} \lesssim 0.01$ suggests that $\mathrm{CeCoIn}_{5}$ is indeed close to 
a QCP. The existence of a $\mathrm{QCP}$ in $\mathrm{CeCoIn}_{5}$ very close to $B_{c 2}$ was also inferred independently from resistivity and magneto-resistivity measurements by Paglione et al. (2003). Surprisingly, when $B_{c 2}$ is suppressed upon alloying $\mathrm{CeCoIn}_{5-x} \mathrm{Sn}_{x}$, the field range where NFL behavior is observed tracks $B_{c 2}$ (Bauer et al., 2005). However, recent resistivity measurements on $\mathrm{CeCoIn}_{5}$ under pressure have shown that $B_{c 2}$ decreases much faster than the quantum critical field, indicating that the two phenomena are not related (Ronning et al., 2006).

While application of hydrostatic pressure drives $\mathrm{CeCoIn}_{5}$ away from quantum criticality (Nicklas et al., 2001), the antiferromagnetic compound $\mathrm{CeRhIn}_{5}$ is driven towards an instability and incipient superconductivity upon application of hydrostatic pressure of $p_{c} \approx$ 14.5 kbar (Fisher et al., 2002; Hegger et al., 2000). The

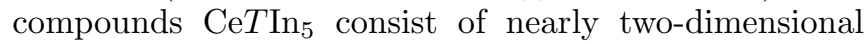
$\mathrm{CeIn}_{3}$ layers, separated by intercalated $T \mathrm{In}_{2}$ layers. However, the magnetic fluctuation of $\mathrm{CeRhIn}_{5}$ as determined from neutron scattering are three-dimensional albeit with some anisotropy (Bao et al., 2002). The pressure-induced transition to superconductivity appears to be of first order, thus "avoiding" a QCP. On the other hand, pronounced deviations from the Fermi-liquid $T^{2}$ dependence of $\rho(T)$ were reported by Muramatsu et al. (2001) to occur over a wide pressure range. Recent studies of the antiferromagnetic/superconducting phase boundary under pressure by Park et al. (2006) and Knebel et al. (2006) revealed a quantum critical line between a phase of coexistence and a purely superconducting phase. Applying additionally a magnetic field, this line is suggested to end in a quantum tetracritical point.

Turning to $\mathrm{CeIrIn}_{5}$, this material exhibits bulk superconductivity below $0.4 \mathrm{~K}$, with $\rho(T)$ vanishing already at $1.2 \mathrm{~K}$ (Petrovic et al., 2001a). The Ce-derived specific heat $C_{m}$, with lattice and nuclear contributions subtracted, exhibits when plotted as $C_{m} / T$ vs. $\ell n T$ a broad hump for magnetic fields applied both along and perpendicular to the $c$ axis. For fields $B \| c$ larger than $12 \mathrm{~T}$, a divergent $C_{m} / T$ for $T \rightarrow 0$ is observed which is interpreted as NFL behavior. Below $0.5 \mathrm{~K}$ with field applied to suppress superconductivity, the resistivity follows a $T^{1.5}$ dependence, also signaling NFL behavior. These findings are interpreted as being due to a field-induced metamagnetic transition (Capan et al., 2004).

\section{5. $\mathrm{YbRh}_{2}\left(\mathrm{Si}_{1-x} \mathrm{Ge}_{x}\right)_{2}$}

The compound $\mathrm{YbRh}_{2} \mathrm{Si}_{2}$ was the first $\mathrm{Yb}$ compound to show pronounced non-Fermi-liquid effects near a magnetic ordering temperature at very low temperature (Trovarelli et al., 2000). It crystallizes in the tetragonal I4/mmm structure (same structure as $\mathrm{CeCu}_{2} \mathrm{Si}_{2}$ ). At high temperatures $T>200 \mathrm{~K}$ the susceptibility follows a Curie-Weiss law with $\mu_{\mathrm{eff}}=4.5 \mu_{\mathrm{B}} /$ Yb-atom but different Weiss temperatures $\theta_{\perp}=-9 \mathrm{~K}$ and $\theta_{\|}=-180 \mathrm{~K}$, due to magnetocrystalline anisotropy, where $\|$ and $\perp$ re-

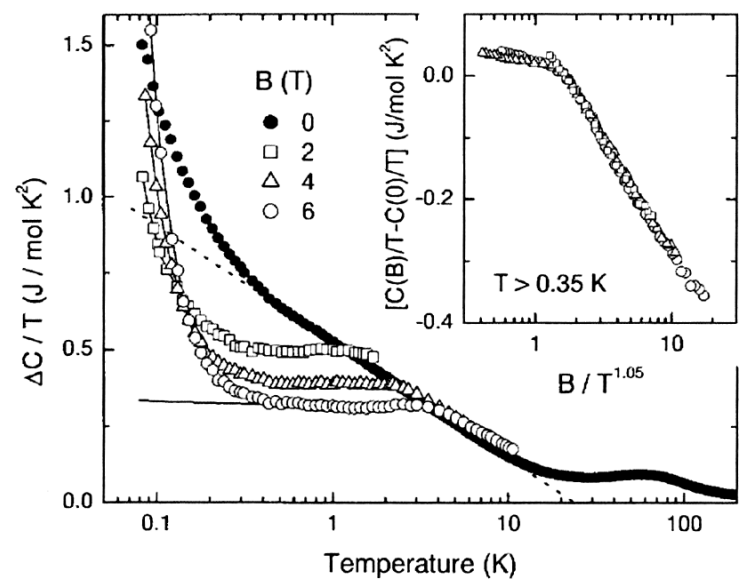

FIG. $15 \mathrm{Yb}$ contribution to the specific-heat coefficient, $\Delta C / T$, of $\mathrm{YbRh}_{2} \mathrm{Si}_{2}$ vs. $T$ (logarithmic scale) for different magnetic fields $B$ applied along the $a$ axis. The dotted straight line represents $\Delta C \sim \ln \left(T_{0} / T\right)$, the horizontal line the $B=6 \mathrm{~T}$ data after subtraction of the hyperfine contribution. The inset demonstrates scaling of the data as $C(B) / T-C(0) / T=f\left(B / T^{\beta}\right)$ with $\beta=1.05$. From Trovarelli et al., 2000.

fer to directions relative to the $c$ axis. At $0.1 \mathrm{~K}$, the large ratio $\chi_{\perp} / \chi_{\|} \approx 100$ classifies $\mathrm{YbRh}_{2} \mathrm{Si}_{2}$ as an easy-plane system, as opposed to the easy-axis system $\mathrm{CeCu}_{6-x} \mathrm{Au}_{x}$.

Maxima in the $a c$ susceptibility $\chi_{\perp}^{a c}(T)$ (Trovarelli et al., 2000) and specific heat $C(T)$ (Gegenwart et al., 2002) as well as a kink in the resistivity (Gegenwart et al., 2002) around $70 \mathrm{mK}$ signal the onset of antiferromagnetic ordering, although to date no neutron scattering data are available to corroborate this assignment. (Recently Gegenwart et al. (2005) reported evidence for ferromagnetic quantum critical fluctuations, see below). Well above the magnetic ordering temperature, i.e., between 0.3 and $10 \mathrm{~K}$, the specific heat varies as $C / T=a \ell n\left(T_{0} / T\right)$ with $a=0.17 \mathrm{~J} / \mathrm{molK}^{2}$ and $T_{0}=24 \mathrm{~K}$ (Fig. 15). The electrical resistivity was found to vary as $\rho=\rho_{0}+A^{\prime} T$ between $20 \mathrm{mK}$ and $1 \mathrm{~K}$, see Fig. 16 (Trovarelli et al., 2000). (The above-mentioned kink at the ordering transition was only found later, likely due to increased sample quality.) These NFL features look at first sight very similar to those in $\mathrm{CeCu}_{6-x} \mathrm{Au}_{x}$ and prompted Trovarelli et al. (2000) to suggest quasi-2d critical fluctuations by analogy with $\mathrm{CeCu}_{6-x} \mathrm{Au}_{x}$. Indeed, there is a surprising scaling when $C / T$ is plotted as function of $\ell n\left(T_{0} / T\right)$ : the data for $\mathrm{YbRh}_{2}\left(\mathrm{Si}_{0.95} \mathrm{Ge}\right)_{0.05}$ and $\mathrm{CeCu}_{5.8} \mathrm{Ag}_{0.2}$ fall on top of each other in the range where $C / T \propto \ln \left(T_{0} / T\right)$ (Küchler et al., 2004). The upturn of $C / T$ below $0.4 \mathrm{~K}$ for $\mathrm{YbRh}_{2} \mathrm{Si}_{2}$ (Fig. 15) can be modelled as $C / T \sim T^{-\alpha}$ with $\alpha \sim 0.3$ (Custers et al., 2003). This upturn suggests the presence of an additional low-energy scale below which the $\ell n T$ behavior of $C / T$ is cut off. It is an open question whether such crossover exists in $\mathrm{CeCu}_{6-x} \mathrm{Au}_{x}$ at correspondingly low reduced temperatures $T / T_{0}$ where $T_{0} \approx$ $6 \mathrm{~K}$ is a factor of 4 smaller. Interestingly, the low- $T$ upturn is suppressed below $0.35 \mathrm{~K}$ when $\mathrm{YbRh}_{2} \mathrm{Si}_{2}$ is diluted 


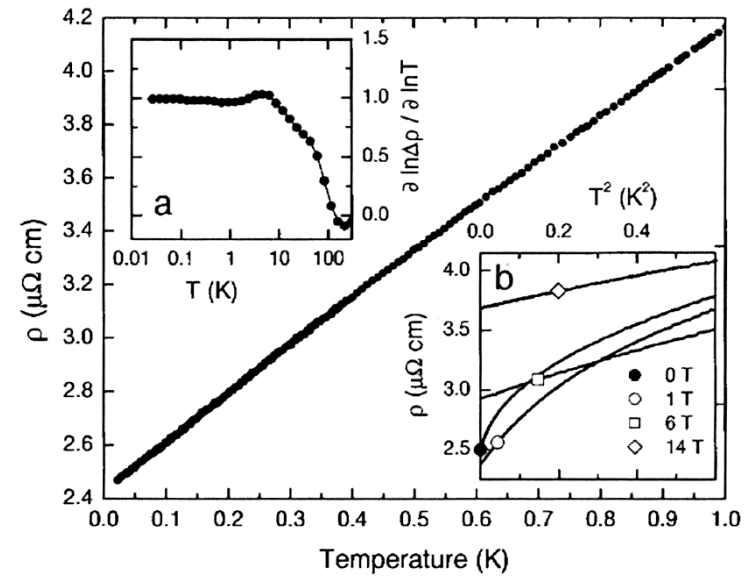

FIG. 16 Low-temperature electrical resistivity $\rho$ of $\mathrm{YbRh}_{2} \mathrm{Si}_{2}$ at $p=0$ measured along the $a$ axis as a function of temperature $T$, obeying $\rho(T)=\rho_{0}+b T^{\alpha}$, with $\alpha \simeq 1$. (a) Temperature dependence of the effective exponent $\epsilon=\partial \ell n \Delta \rho / \partial \ell n T$ with $\Delta \rho=\rho-\rho_{0}$. (b) $\rho(T)$ plotted as $\rho$ vs. $T^{2}$, for $B \leq 14 \mathrm{~T}$ applied along the $c$ axis. From Trovarelli et al., 2000.

with La (Ferstl et al., 2005): For $\mathrm{Yb}_{0.95} \mathrm{La}_{0.05} \mathrm{Rh}_{2} \mathrm{Si}_{2}$, $C / T \sim \ln \left(T_{0} / T\right)$ is observed down to $0.35 \mathrm{~K}$, the lowest $T$ measured.

Measurements on $\mathrm{YbRh}_{2}\left(\mathrm{Si}_{1-x} \mathrm{Ge}_{x}\right)_{2}$ with a nominal Ge concentration $x=0.05$ showed that the magnetic ordering is suppressed down to $20 \mathrm{mK}$ where a rather broad maximum in the specific heat is observed (Custers et al., 2003). Above $100 \mathrm{mK}$, the $C / T$ curves for $x=0$ and $x=0.05$ are nearly identical, indicating that the $T^{-0.3}$ upturn is not associated with classical short-rangeorder effects in the proximity to magnetic ordering.

A further interesting discovery for $\mathrm{YbRh}_{2} \mathrm{Si}_{2}$, following the prediction by Zhu et al. (2003) discussed in Sec. III.D, was the observation of a divergent Grüneisen parameter $\Gamma$ near the QCP by Küchler et al. (2003). As shown in Fig. 17, the $\Gamma$ data for $\mathrm{YbRh}_{2}\left(\mathrm{Si}_{0.95} \mathrm{Ge}_{0.05}\right)_{2}$ follow a $T^{-0.7}$ dependence at lowest temperatures, i.e., between $50 \mathrm{mK}$ and $0.6 \mathrm{~K}$, with a slightly steeper slope approaching an exponent -1 at higher $T$ (Fig. 17). The $T^{-0.7}$ dependence of $\Gamma$ at low $T$ is certainly weaker that $T^{-1}$ predicted for the $3 \mathrm{~d}$ LGW scenario (and actually observed for $\mathrm{CeNi}_{2} \mathrm{Ge}_{2}$ by Küchler et al. 2003), but also weaker than $T^{-1}(\ell n \ell n T) /(\ell n T)$ expected for a $2 \mathrm{~d} \mathrm{LGW}$ model. This observation may support the idea that degrees of freedom other than magnetism become critical at the QPT, see Sec. III.I. However, the data would imply critical exponents with $1 /(z \nu)=0.7$ (at least if the transition is below its upper critical dimension), which has not been verified by other measurements.

Rather detailed experiments were carried out to study the behavior of $\mathrm{YbRh}_{2}\left(\mathrm{Si}_{1-x} \mathrm{Ge}_{x}\right)_{2}$ in magnetic fields, exploring the field tuning of a quantum critical point, as previously done for the $\mathrm{CeCu}_{6-x} \mathrm{Ag}_{x}$ and $\mathrm{CeCu}_{6-x} \mathrm{Au}_{x}$ series. The magnetic $B-T$ phase diagram of $\mathrm{YbRh}_{2} \mathrm{Si}_{2}$ shown in Fig. 18 is universal with respect to the different field orientations when the field axes are scaled appropriately, similar to the behavior shown before for the anisotropic easy-axis system $\mathrm{CeCu}_{6-x} \mathrm{Au}_{x}$ (Schlager et al., 1993). While for high magnetic fields constant Kadowaki-Woods ratios $A / \gamma_{0}^{2}$ and $A / \chi_{0}^{2}$ typical for a Fermi liquid are observed, the ratio $A / \gamma_{0}^{2} \sim\left(B-B_{c}\right)^{-0.33}$ appears to diverge close to $B_{c}$, as has been shown for $\mathrm{YbRh}_{2}\left(\mathrm{Si}_{0.95} \mathrm{Ge}_{0.05}\right)_{2}$ by Custers et al. (2003). In this alloy, $B_{c}^{\perp}=0.027 \mathrm{~T}$ only and $\gamma_{0}$ diverges as $\gamma_{0} \sim$ $\left(B-B_{c}\right)^{-0.33}$. Yet, the coefficient $A$ of the $T^{2}$ resistivity diverges more strongly. For a $2 \mathrm{~d}$ LGW scenario, $A / \gamma_{0}^{2}$ diverges much faster, i.e., $A / \gamma_{0}^{2} \sim 1 /\left(B-B_{c}\right)$ with logarithmic corrections.

As discussed in detail in Sec. III.I, one of the central questions for quantum critical points in HFS is whether the Kondo effect breaks down at the critical point and whether the Fermi volume changes abruptly at the second-order transition (Coleman et al., 2001; Senthil, Sachdev, and Vojta, 2005; Si, Smith, and Ingersent, 1999; Si et al., 2003). Indeed, Paschen et al. (2004) found indications of such a behavior in measurements of the Hall effect across the field-driven transition in $\mathrm{YbRh}_{2} \mathrm{Si}_{2}$. In Fig. 19 the Hall constant $R_{H}$ is shown for a geometry (see inset) where the field $B_{2}$ driving the QPT is parallel to the current $I$, and an additional small probing field $B_{1}$ perpendicular to $I$ induces the Hall voltage. As can be seen in Fig. 19, $R_{H}$ varies quite strongly, consistent with a change of the Fermi volume by one electron per unit cell (assuming a free-electron single-band picture). For lower $T$ the crossover gets sharper, consis-

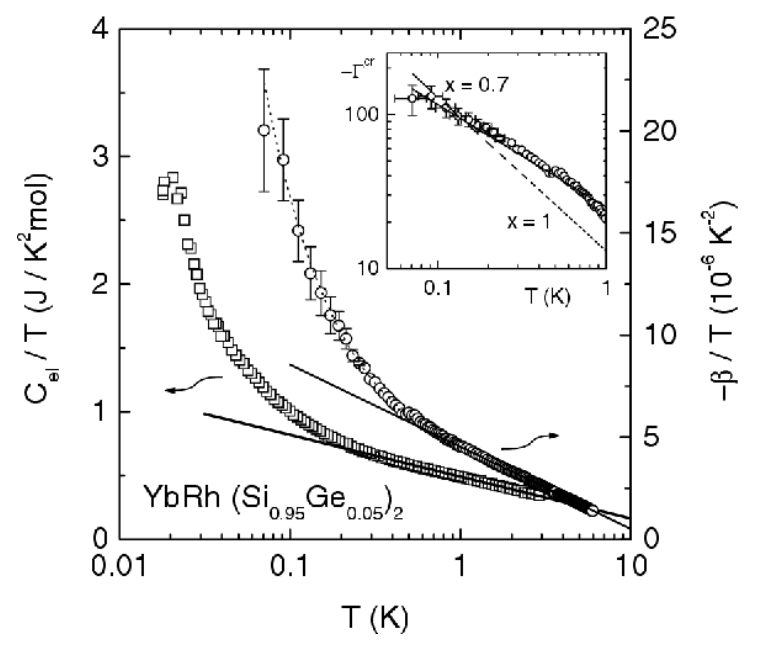

FIG. 17 Electronic specific heat as $C_{e l} / T$ (left axis) and volume thermal expansion as $-\beta / T$ (right axis) vs $T$ (on a logarithmic scale) for $\mathrm{YbRh}_{2}\left(\mathrm{Si}_{0.95} \mathrm{Ge}_{0.05}\right)_{2}$ at $B=0$. The solid lines indicate $\ln \left(T_{0} / T\right)$ dependencies with $T_{0}=30 \mathrm{~K}$ and $13 \mathrm{~K}$ for $C_{e l} / T$ and $-\beta / T$, respectively. The dotted line represents $-\beta / T=a_{0}+a_{1} / T$ with $a_{0}=3.4 \times 10^{-6} \mathrm{~K}^{-2}$ and $a_{1}=$ $1.34 \times 10^{-6} \mathrm{~K}^{-2}$. The inset displays the log-log plot of $\Gamma_{\mathrm{cr}}(T)$ with $\Gamma_{\mathrm{cr}}=V_{m} / \kappa_{T} \cdot \beta_{\mathrm{cr}} / C_{\mathrm{cr}}$ using $\kappa_{T}=5.3 \times 10^{-12} \mathrm{~Pa}^{-1}$, $\beta_{\mathrm{cr}}=\beta(T)+a_{0} T$, and $C_{\mathrm{cr}}=C_{e l}(T)$. The solid and dotted lines represent $\Gamma_{\mathrm{cr}} \propto 1 / T^{x}$ with $x=0.7$ and $x=1$, respectively. From Küchler et al., 2003. 


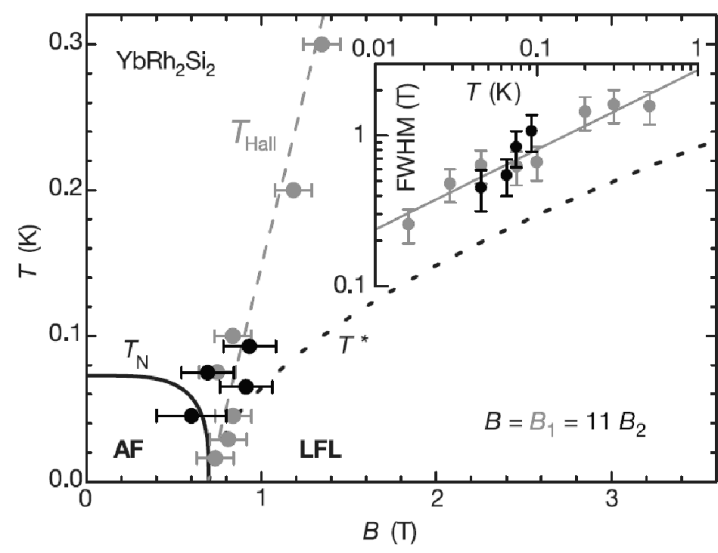

FIG. 18 Temperature-field phase diagram of $\mathrm{YbRh}_{2} \mathrm{Si}_{2}$. Full and dotted black curves represent the field dependence of the Néel temperature $T_{\mathrm{N}}$ and the crossover temperature $T^{*}$ below which the resistivity exhibits a Fermi-liquid $T^{2}$ dependence. Symbols present the crossover field where the Hall resistivity changes the slope. Light symbols: $B=B_{1}$ parallel to the $c$ axis, dark symbols: crossed-field experiment with the tuning field $B=B_{2}$ perpendicular to the $c$ axis. The relation $B=$ $B_{1}=11 B_{2}$ reflects the magnetic anisotropy of $\mathrm{YbRh}_{2} \mathrm{Si}_{2}$. Inset: Width of the transition at $B_{c}$ vs. $T$, indicating that it vanishes as $T \rightarrow 0$. From Paschen et al., 2004.

tent with a scaling of the half-width with $\sqrt{T}$, see dark symbols in the inset of Fig. 18. Paschen et al. (2004) have also measured the Hall effect in a different geometry, where the field driving the QPT is identical to the field which induces the Hall voltage. This geometry is, however, more difficult to interpret, as the resulting differential Hall resistivity is expected to jump even for a standard SDW quantum critical point. (Note that such a jump is expected to trace $T_{N}$, opposite to what is observed in $\mathrm{YbRh}_{2} \mathrm{Si}_{2}$.)

By using fits to the theory of the anomalous Hall effect arising from skew-scattering (Fert and Levy, 1987),

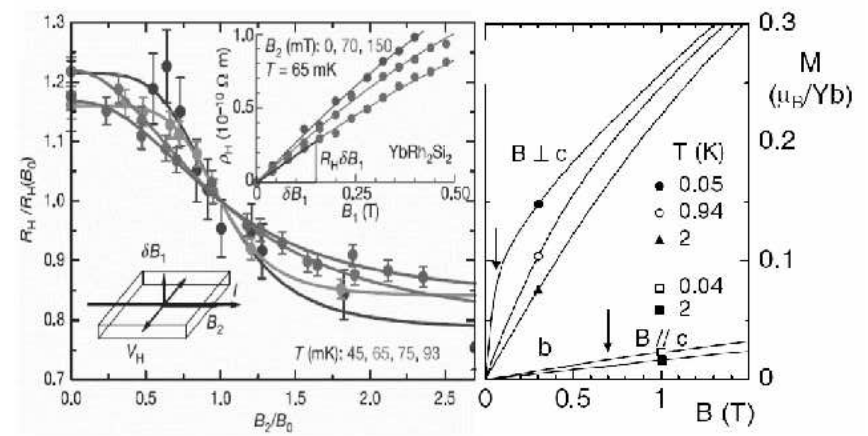

FIG. 19 Hall effect and magnetizations of $\mathrm{YbRh}_{2} \mathrm{Si}_{2}$. Left: Hall effect as a function of a magnetic field parallel to the current. From Paschen et al., 2004. The crossover at the critical field $B_{0}=60 \mathrm{mT}$ gets sharper towards lower $T$. Right: Magnetization as a function of $B$, arrows indicate the critical field. From Gegenwart et al., 2002.
Paschen et al. (2004) concluded that the former is sufficiently small to be either subtracted or neglected (depending on the geometry). In this case the extrapolated jump of the Hall coefficient at $T=0$ would indeed prove rather unambiguously a jump of the Fermi volume at a second-order phase transition, certainly a very spectacular result. To establish this firmly, it would be important to track the sharpening of the crossover in Fig. 19 towards lower temperature, and check for similar effects in other systems as well. When interpreting the crossover at finite $T$ one may also have to take into account that $\mathrm{YbRh}_{2} \mathrm{Si}_{2}$ is almost ferromagnetic (see below). Even the tiny critical field of $60 \mathrm{mT}$ already induces a sizable magnetization of almost $0.1 \mu_{B}$ per $\mathrm{Yb}$ which is strongly temperature dependent, see right panel of Fig. 19. Such a magnetization could at least in principle lead to large changes of the Fermi surface and therefore of the Hall effect. The change in $\rho_{x y}(B)$ has also been interpreted in terms of quantum critical valence fluctuations (Norman, 2005).

We now turn to the issue of critical fluctuations in $\mathrm{YbRh}_{2} \mathrm{Si}_{2}$ near the QPT. Unfortunately, neither elastic nor inelastic neutron scattering data exist to date which could identify the type of magnetic order and the nature of the critical fluctuations. Hence for a microscopic view one has to rely on local probes such as NMR and ESR, and on macroscopic magnetization data. Ishida et al. (2002) reported ${ }^{29}$ Si NMR data on aligned single crystals of $\mathrm{YbRh}_{2} \mathrm{Si}_{2}$ in fields parallel to the easy axis. While the nuclear spin-lattice relaxation rate divided by $T, 1 / T_{1} T$, was found to vary as $\sim T^{-1 / 2}$ at small magnetic field of $B_{\perp}=0.15 \mathrm{~T}$ down to the lowest temperature $(\sim 50 \mathrm{mK})$, the Knight shift $K$ first also increases towards $T \rightarrow 0$ but turns over to a constant value at $T \approx 200 \mathrm{mK}$ in the same field. While $K$ probes the uniform static susceptibility $\chi^{\prime}(\mathbf{q}=0), 1 / T_{1} T$ gives information over the imaginary part of the q-averaged dynamical spin susceptibility $\chi^{\prime \prime}(\mathbf{q}, \omega)$ at the (quasi-static) frequency corresponding to the nuclear Zeeman splitting. The difference between $K$ and $1 / T_{1} T$ has been attributed to the presence of finite$q$ critical fluctuations. For higher fields, $K$ and $1 / T_{1} T$ tend to a $T$-independent value and track each other, i.e., the Korringa relation $1 / T_{1} T \sim S K^{2}$ is approximately fulfilled between 0.25 and $2.4 \mathrm{~T}$. The strong deviation of $S$ from the free-electron value $S_{0}=\pi \hbar \gamma_{n}^{2} k_{B} / \mu_{\mathrm{B}}^{2}$, i.e., $S \approx 0.1 S_{0}$ as measured at $100 \mathrm{mK}$, suggests the presence of dominant $q=0$, i.e., ferromagnetic fluctuations.

Similarly the approximately constant ratio $A / \chi_{0}^{2}$ discussed above and observed for $B \gtrsim 2 B_{c}$ is not easy to understand for dominant AFM fluctuations. In addition, the strong increase of the Wilson ratio $\chi_{0} / \gamma_{0}$ by a factor of up to 30 compared to the free-electron value "highlights the importance of FM fluctuations in the approach to the QCP" (Gegenwart et al., 2005). However, by comparing tendencies to ferro- and antiferromagnetism in a Ge-doped sample with an undoped one, Gegenwart et al. (2005) also suggested that ferromagnetic tendencies are "not directly correlated to the AFM QCP". Recent 
magneto-striction data, showing the vanishing of several crossover energies at the QCP, have also been discussed in relation to uniform magnetism (Gegenwart et al., 2007).

There is a further intriguing observation in $\mathrm{YbRh}_{2} \mathrm{Si}_{2}$, possibly related to ferromagnetic fluctuations, i.e., the observation of an ESR signal by Sichelschmidt et al. (2003) that can be ascribed to a $\mathrm{Yb}^{3+}$ resonance. This observation is very surprising because the large Kondo temperature $T_{\mathrm{K}} \approx 25 \mathrm{~K}$ is associated with a very high spin-fluctuation rate that would according to current wisdom render any ESR signal below $T_{\mathrm{K}}$ unobservable. However, Sichelschmidt et al. (2003) observe an ESR $\mathrm{Yb}^{3+} 4 f^{13}$ signal well below $T_{\mathrm{K}}$ that suggests that at least $60 \%$ of the $\mathrm{Yb}^{3+}$ ions in $\mathrm{YbRh}_{2} \mathrm{Si}_{2}$ contribute.

It is tantalizing that the $T^{-0.7}$ dependence of $\Gamma$ and the $T^{-0.3}$ dependence of $C / T$ in $\mathrm{YbRh}_{2}\left(\mathrm{Si}_{1-x} \mathrm{Ge}_{x}\right)_{2}$ at very low temperatures, but above the ordering temperature correspond to a $2 \mathrm{~d}$ ferromagnetic $(z=3)$ scenario of a conventional LGW QPT. A speculative view could be that ferromagnetic planes are the source of the strong FM component of the fluctuations that have been discussed above, until the magnetic transition intervenes, perhaps induced by a weak antiferromagnetic interplane coupling.

\section{B. Quantum critical behavior of itinerant transition-metal magnets}

Intermetallic transition-metal compounds have been traditionally viewed as systems close to a magnetic instability, and the LGW model has been formulated in the self-consistently renormalized (SCR) theory of spin fluctuations (Sec. III.E) to describe these systems (Lonzarich and Taillefer, 1985; Moriya, 1985). While MnSi and $\mathrm{ZrZn}_{2}$ have been outstanding examples under intense investigation recently - to be discussed below there have been a number of other systems that should be mentioned.

The nearly ferromagnetic metal $\mathrm{Ni}$ can be driven to static ferromagnetic order by introducing $\mathrm{Pd}$, with a $\mathrm{Pd}$ critical concentration of $x=0.026$. Here, the resistivity $\rho$ follows a $T^{5 / 3}$ dependence (Nicklas et al., 1999). This $T$ dependence, as well as the $T_{\mathrm{C}} \sim\left(x-x_{c}\right)^{3 / 4}$ dependence are in accord with the FM LGW model. Likewise, the specific heat $\Delta C$ (after subtraction of the phonon contribution) shows $\Delta C / T \sim \ln \left(T_{0} / T\right)$ at $x_{c}$. The ferromagnet $\mathrm{Ni}_{3} \mathrm{Al}$ has been investigated with resistivity measurements under pressure up to $10 \mathrm{GPa}$ (Niklowitz et al., 2005). The critical pressure $p_{c}$ where $T_{c} \rightarrow 0$ is estimated to be around $8 \mathrm{GPa}$. The $T$-dependent part $\Delta \rho$ of the resistivity does not reveal pronounced critical behavior, the exponent $\alpha$ depends on $T$ and varies for $3.2 \mathrm{GPa}$ between 1.9 and 1.5 at $1 \mathrm{~K}$ and $5 \mathrm{~K}$, respectively, and smoothly shifts to lower values with increasing $p$. Below $1 \mathrm{~K}$, the data can be approximated by $\Delta \rho=A T^{2}$, and $A$ exhibits a rather sharp maximum at $p_{c}$. This behavior has been tentatively associated with a first-order transition near $p_{c}$ (Niklowitz et al., 2005). $\mathrm{YMn}_{2}$ is an itinerant antifer- romagnet with a first-order transition around $T_{\mathrm{N}} \approx 100 \mathrm{~K}$ (Freltoft, 1988). On the other hand, Mössbauer measurements under pressure indicated a continuous reduction of the ordered magnetic moment (Block, Abd-Elmeguid, and Micklitz, 1994) suggesting a second-order QCP.

We finally mention experiments on an, albeit complex, elemental metal, $\beta$-Mn (Stewart et al., 2002), where the resistivity varies as $\Delta \rho \sim T^{3 / 2}$ between $5 \mathrm{~K}$ (the lowest temperature measured) and $25 \mathrm{~K}$, suggesting the proximity to a AFM QCP. The spin fluctuations, however, defy a simple interpretation in terms of a unique scaling. As already mentioned at the beginning of this chapter, space limitations inhibit the discussion of QCP in transition metal oxides, such as high- $T_{c}$ cuprates, manganites, or $\mathrm{Sr}_{3} \mathrm{Ru}_{2} \mathrm{O}_{7}$.

\section{1. $\mathrm{Cr}_{1-x} \mathrm{~V}_{x}$}

Before diving into ferromagnetic systems, let us discuss the material $\mathrm{Cr}_{1-x} \mathrm{~V}_{x}$, which derives from the itinerant antiferromagnet $\mathrm{Cr}$, and is one of the very few "nonKondo" systems which can be driven through an antiferromagnetic QPT.

In particular, the Hall effect near the QCP in $\mathrm{Cr}_{1-x} \mathrm{~V}_{x}$ was investigated with concentration tuning $\left(x_{c}=0.035\right)$ (Yeh et al., 2002), and by pressure tuning of a sample with $x=0.032$ close to $x_{c}$ (Lee et al., 2004). For this concentration $T_{\mathrm{C}}=52 \mathrm{~K}$ compared to $T_{\mathrm{C}}=311 \mathrm{~K}$ for pure Cr. While the transition upon concentration variation appears very sharp (i.e. almost of first order), using a finely spaced pressure tuning reveals it to be continuous. The decrease of the inverse Hall constant, $R_{H}^{-1}$, upon opening of the spin-density wave gap, tracks the increase of $\rho$ above the paramagnetic background resistivity. The Hall effect evolution was interpreted in terms of an almost nested Fermi surface (Norman et al., 2003). The available data on $\mathrm{Cr}_{1-x} \mathrm{~V}_{x}$ appear to be consistent with a $3 \mathrm{~d}$ LGW scenario of the transition.

\section{2. $\mathrm{MnSi}$}

The itinerant-electron magnet MnSi orders below $T_{\mathrm{C}}=$ $29.5 \mathrm{~K}$ in a spiral magnetic structure with a long pitch of about $175 \AA$. Early on it was realized (Bak and Jensen, 1980; Nakanishi et al., 1980) that the helical order arises as the cubic B20 structure (space group P2 ${ }_{1} 3$ ) lacks inversion symmetry. Therefore the weak spin-orbit interactions assume a Dzyaloshinsky-Moriya form $\int \mathbf{S} \cdot(\nabla \times \mathbf{S}) d \mathbf{r}$ which twists the ferromagnetic alignment into a helix. The ordered moment of about $0.4 \mu_{B} / \mathrm{Mn}$-atom is sizable for an itinerant magnet. With the possible exception of high-frequency optical-conductivity experiments (Mena et al., 2003), the ground state below $T_{\mathrm{C}}$ seems to be a standard three-dimensional weakly spin-polarized Fermi liquid (Fawcett et al., 1970; Ishikawa et al., 1985; Taillefer, Lonzarich, and Strange, 1986). MnSi, being almost 


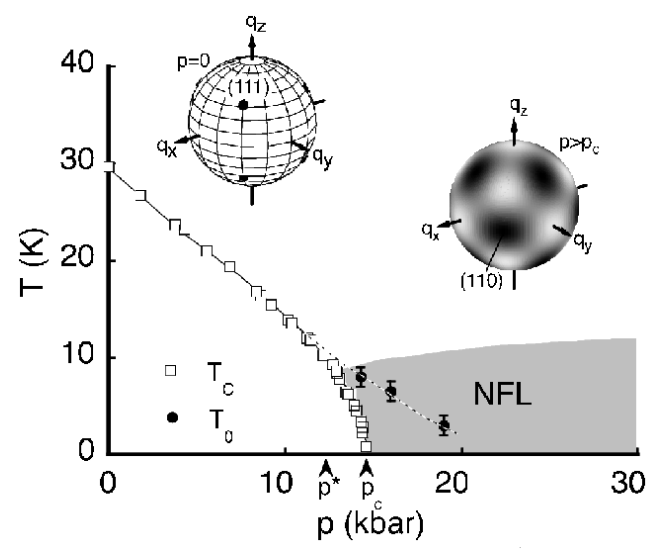

FIG. 20 Temperature-pressure phase diagram of MnSi. Above $p^{*}$, the transition is first order, see Fig. 21 and vanishes at $p_{c}$. In the shaded region, the resistivity shows an anomalous temperature dependence, $\Delta \rho(T) \sim T^{3 / 2}$. The insets qualitatively show main features of the neutron scattering intensity, see text. From Pfleiderer et al., 2004.

a ferromagnet, played an important role in the development of spin-fluctuation theory for complex transition metal compounds (Lonzarich and Taillefer, 1985; Moriya, 1985), see Sec. III.E.

When pressure is applied (see Fig. 20) the magnetic order is suppressed (Thompson, Fisk, and Lonzarich, 1989) and vanishes above $p_{c}=14.6 \mathrm{kbar}$. Above this pressure, the resistivity shows an anomalous power-law, $\Delta \rho \sim T^{3 / 2}$ (Doiron-Leyraud et al., 2003; Pfleiderer et al., 2001b). It has to be emphasized that this power-law holds over almost three decades in temperature from about $6 \mathrm{~K}$ down to a few $\mathrm{mK}$ and over a large pressure range.

Therefore the question arises whether the anomalous resistivity can be associated with critical fluctuations. For example, due to the long pitch of the spiral, one might expect a quantum critical behavior similar to that

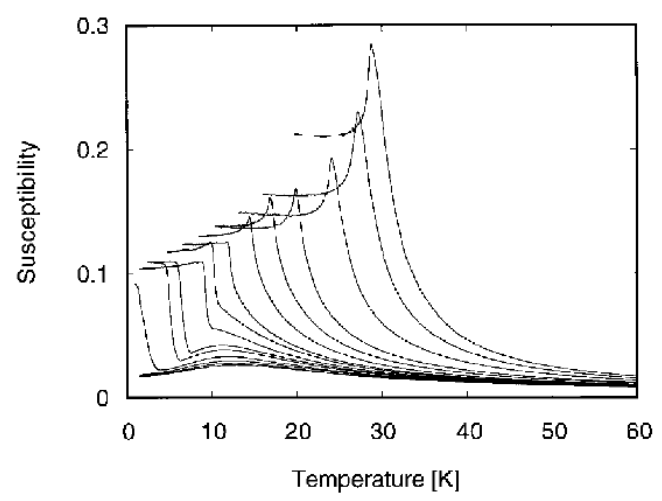

FIG. 21 Magnetic susceptibility of MnSi vs. $T$ at different pressures (ambient, 1.80, 3.80, 6.90, 8.60, 10.15, 11.25, 12.15, $13.45,13.90,14.45,15.20,15.70$, and 16.10 kbar going down, starting from the top curve at $30 \mathrm{~K}$ ). Close to the critical pressure, $\chi(T \rightarrow 0)$ shows a pronounced jump indicative of a first-order phase transition. From Pfleiderer et al., 1997.

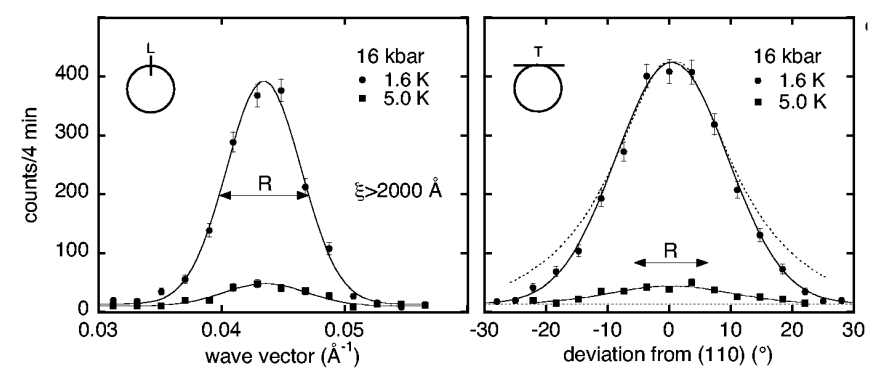

FIG. 22 Scans of the elastic neutron scattering intensity in $\mathrm{MnSi}$ at $16 \mathrm{kbar}$ perpendicular (left) and parallel (right) to the surface of the sphere shown in Fig. 20 [in (110) direction]. From Pfleiderer et al., 2004.

of a ferromagnet with $\Delta \rho \sim T^{5 / 3}$ (Sec.II.E), not too far from the observed $\Delta \rho \sim T^{3 / 2}$. There are two main arguments against such an interpretation. First, the anomalous transport is observed far away from the putative quantum critical point. While a pressure of $14.6 \mathrm{kbar}$ is sufficient to suppress $T_{\mathrm{C}}$ from almost $30 \mathrm{~K}$ down to zero, an up to threefold pressure increase is not sufficient to recover Fermi-liquid behavior at temperatures of the order of $50 \mathrm{mK}$. (Recent measurements by Pedrazzini et al. (2006) indicate, however, that for $p>3 p_{c}$ the exponent changes and Fermi-liquid behavior might be recovered at the lowest temperatures.) More relevant is a second argument: Susceptibility measurements, see Fig. 21, show that close to $p_{c}$ the phase transition is of first order and critical fluctuations should be absent. Indeed, Pfleiderer et al. (2001b) and Doiron-Leyraud et al. (2003) have tried to estimate the consequence of such a first-order transition quantitatively and concluded that within a standard spin-fluctuation scenario a $T^{2}$ resistivity should be observable below 1 or $2 \mathrm{~K}$, in contradiction to experiment. The situation is, however, complicated by the fact that not all experimental probes show indications of a strong first-order transition. For example, the hysteretic part of the signal in elastic neutron scattering (Pfleiderer et $a l ., 2004)$ is rather small close to $p_{c}$, and on the ordered side of the phase diagram the $A$ coefficient of the $T \rightarrow 0$ resistivity $\rho=\rho_{0}+A T^{2}$ seems to diverge upon approaching $p_{c}$ indicative of a second-order transition (possibly of percolative type).

A consistent explanation of the precise nature of the quantum phase transition is presently lacking. Theoretically, it has been argued by Schmalian and Turlakov (2004) that strong fluctuations of the direction of the ordering vector generically drive the QPT towards first order. If quantum critical fluctuations can be ruled out as the origin of the NFL behavior, the alternative scenario is the existence of an extended new NFL phase which is stable against changes of pressure and temperature in a sizable region. This would be remarkable for a threedimensional cubic system where disorder effects should be negligible due to high-quality single crystals with a mean free path of several thousand $\AA$.

Recent elastic neutron scattering experiments by Pflei- 
derer et al. (2004) have surprisingly shown that even above $p_{c}$ the magnetic order has not vanished completely. While for $p<p_{c}$ one observes Bragg reflections in the $\langle 111\rangle$ directions with an ordering vector of $|\mathbf{Q}| \approx 0.037 \AA^{-1}$ (increasing under pressure), one finds for $p \gtrsim p_{c}$ and below a crossover temperature $T_{0}$ (shown in Fig. 20) a strange type of "partial order": the neutron scattering intensity is concentrated on the surface of a sphere in reciprocal space with a radius $Q \approx 0.0437 \AA^{-1}$. Resolution-limited scans along the radial direction of this sphere, see Fig. 22, reveal that the helical order survives above $p_{c}$ on length scales of at least $\sim 2000 \AA$. The broad distribution of the $\mathbf{Q}$ vector in tangential direction on the surface of the sphere (Fig. 22 and inset of Fig. 20) implies the absence of long-range order: the helices have lost their directional order. Interestingly, the total intensity of the elastic neutron signal is comparable to that at ambient pressure implying that a sizable fraction of the local order survives above $p_{c}$.

Likewise, zero-field ${ }^{29} \mathrm{Si} \mathrm{NMR}$ experiments by $\mathrm{Yu}$ et al. (2004) show this local order below $T_{0}$. The broadening of the distribution of NMR frequencies shown in Fig. 23 is consistent with a picture of intrinsic phase inhomogeneities above $p^{*}$ (note that the powdered samples used in this experiment may have extra pinning centers). Several theoretical attempts have been made to identify possible unconventional order-parameter structures and lattices of defects (Binz, Vishwanath, and Aji, 2006; Fischer, Shah, and Rosch, 2007; Rößler, Bogdanov, and Pfleiderer, 2006) which may serve as a starting point to understand the observed partial order. The role of disorder and phase inhomogeneities in this extremely clean system remains, however, presently unclear.

The question arises whether the anomalous partial order or a new QCP, associated with the vanishing of the crossover scale $T_{0}$ (see Fig. 20), can be the origin of the

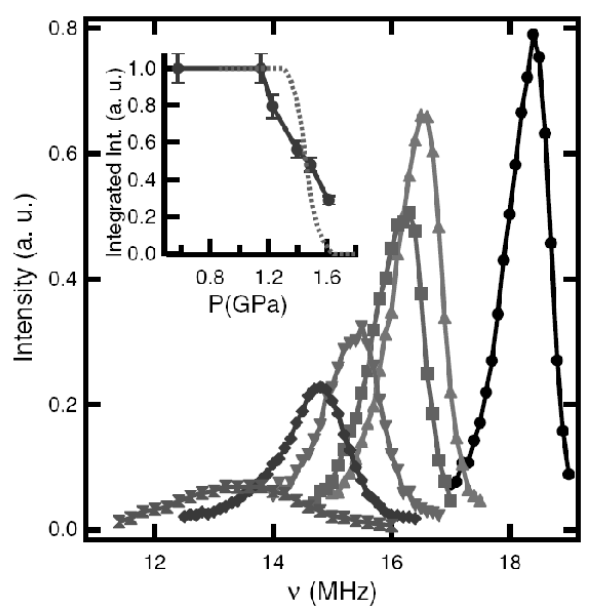

FIG. 23 Distribution of ${ }^{29} \mathrm{Si}$ NMR frequencies in $\mathrm{MnSi}$ for pressures $p=0.58,1.15,1.23,1.40,1.49$, and $1.62 \mathrm{GPa}$ (right to left). Inset: The corresponding total intensities. From Yu et al., 2004. anomalous transport properties in MnSi. For example, anomalously soft Goldstone modes of a helimagnet give rise to singular behavior of thermodynamic and transport quantities (Belitz, Kirkpatrick, and Rosch, 2006a). However, for clean systems $\Delta \rho \sim T^{5 / 2}$ rather than the observed $T^{3 / 2}$ has been predicted (Belitz, Kirkpatrick, and Rosch, 2006b) [neglecting, however, the role of spinorbit coupling (Fischer and Rosch, 2004)]. A challenge is to understand the role of the scale $T_{0}$ above which the partial order seems to vanish. Surprisingly, no signatures of $T_{0}$ can be seen in transport, $\Delta \rho \sim T^{3 / 2}$ both above and below $T_{0}$. This is unexpected as at $T_{\mathrm{C}}$, when long-range order sets in, the resistivity shows a pronounced kink and a strong reduction. It has therefore been speculated by Pfleiderer et al. (2004) that the partial order survives on intermediate length and time scales and gives rise to an anomalous scattering of electrons (e.g. from fluctuating topological defects) even above $T_{0}$ everywhere in the shaded area in Fig. 20. Within this interpretation $T_{0}$ is just a freezing temperature below which the partial order gets static and is observable by elastic neutron scattering or NMR. As both inhomogeneities and fluctuating order on intermediate time and length scales are expected to be of importance in other quantum critical systems as well, a further understanding of the NFL behavior in MnSi is highly desirable.

\section{3. $\mathrm{ZrZn}_{2}$}

$\mathrm{ZrZn}_{2}$ is an itinerant electron ferromagnet. It has a cubic structure (C15, Fd $\overline{3} \mathrm{~m})$. The small ordered magnetic moment $\mu_{0}=0.17 \mu_{B} / \mathrm{Zr}$-atom contrasts with the large Curie-Weiss moment $\mu_{\text {eff }}=1.9 \mu_{B} /$ Zr-atom. This difference and the low Curie temperature $T_{\mathrm{C}}=28.5 \mathrm{~K}$ classify the system as weak itinerant magnet. It has long been known that $T_{\mathrm{C}}$ and $\mu_{0}$ can be suppressed by hydrostatic pressure while $\mu_{\text {eff }}$ stays practically constant (Huber et $a l .$, 1975). However, the detailed $T_{\mathrm{C}}(p)$ dependence depends strongly on the purity of the sample. $\mathrm{ZrZn}_{2}$ has long been considered a candidate for $p$-wave superconductivity (Fay and Appel, 1980). Recent experiments reported superconductivity in very pure $\mathrm{ZrZn}_{2}$ (Pfleiderer et al., 2001a) which was, however, subsequently shown to arise from a surface layer treated by spark erosion (Yelland et al., 2005). Magnetization experiments on very pure crystals $(\mathrm{RRR} \approx 100)$ suggest that $\mu_{0}$ and $T_{\mathrm{C}}$ decrease linearly and drop discontinuously at a pressure $p_{c}$ $=16.5 \mathrm{kbar}$ as shown in Fig. 24 (Uhlarz, Pfleiderer, and Hayden, 2004). This is in contrast with the continuous transition observed as a function of temperature at $p=0$. De Haas-van Alphen experiments under pressure show that the exchange splitting between two nearly spherical Fermi-surface sheets that can be identified with minority and majority spin sheets, respectively, decreases with increasing pressure (Kimura et al., 2004).

The magnetization data as function of $p$ and $B$ suggest the existence of two distinct ferromagnetic phases 


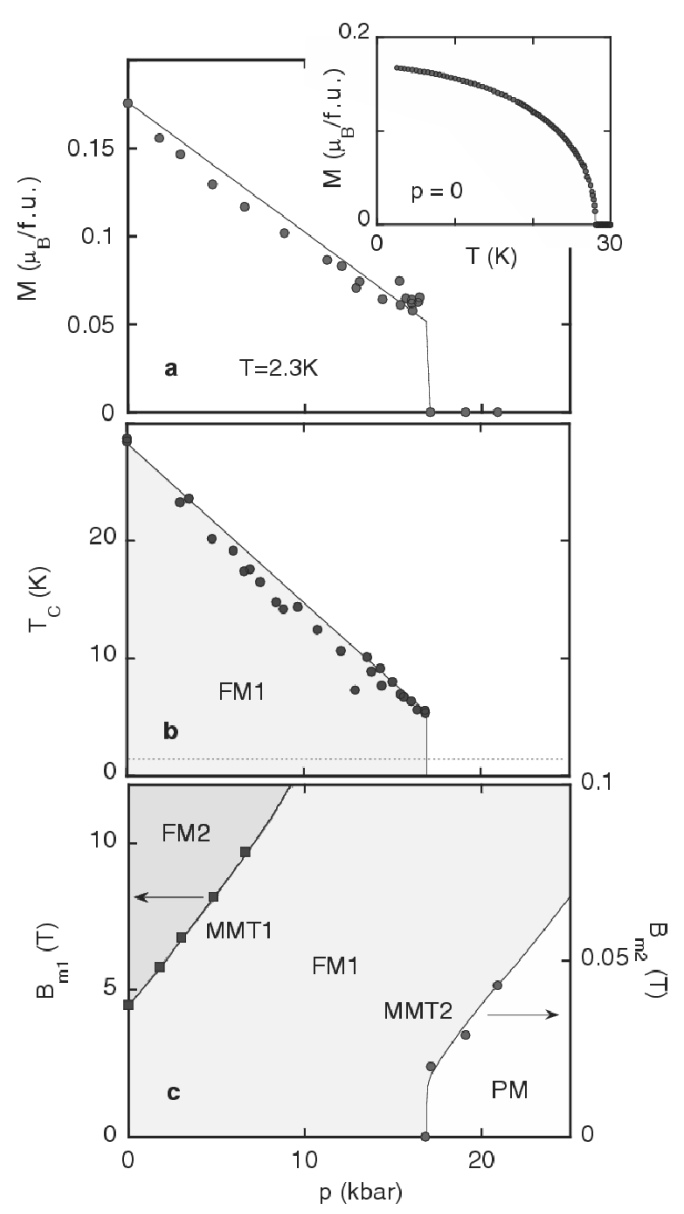

FIG. 24 Pressure dependence (a) of the spontaneously ordered moment $M$ in units of $\mu_{B}$ per formula unit and (b) of the Curie temperature $T_{\mathrm{C}}$ of $\mathrm{ZrZn}_{2}$. Dashed line in (b) indicates the lowest temperature measured. (c) $(B, P)$ phase diagram at low $T$ with two ferromagnetic phases FM1 and FM2 and paramagnetic phase PM, separated by phase lines MMT1 and MMT2. Inset: Continuous decrease of $M$ as a function of T. From Uhlarz, Pfleiderer, and Hayden, 2004.

FM1 and FM2. This rather complicated phase diagram may be a consequence of a double-peak structure in the electronic density of states close to $E_{F}$ (Sandeman et al., 2003), which also possibly causes the first-order transition near $p_{c}$. On more general grounds, the transition close to the QPT may be generically of first order due to a coupling of long-wavelength magnetic modes to particlehole excitations, see Sec. III.H.1. In this scenario (Belitz, Kirkpatrick, and Rollbühler, 2005) the second-order transition at low $T$ and zero field becomes first order at a tricritical point, while second-order phase transition lines seam a surface of first-order transitions in the threedimensional $(p, B, T)$ space, see Fig. 4 in Sec. III.H.1.

A systematic study of $\mathrm{Zr}_{1-x} \mathrm{Nb}_{x} \mathrm{Zn}_{2}$ (Fig. 25) revealed a divergence of $\chi$ for $T \rightarrow 0$ at a critical concentration $x_{c}=0.083$ where $\chi \sim T^{-4 / 3}$ (Sokolov et al., 2006). The spontaneous moment vanishes linearly when $x \rightarrow x_{c}$.

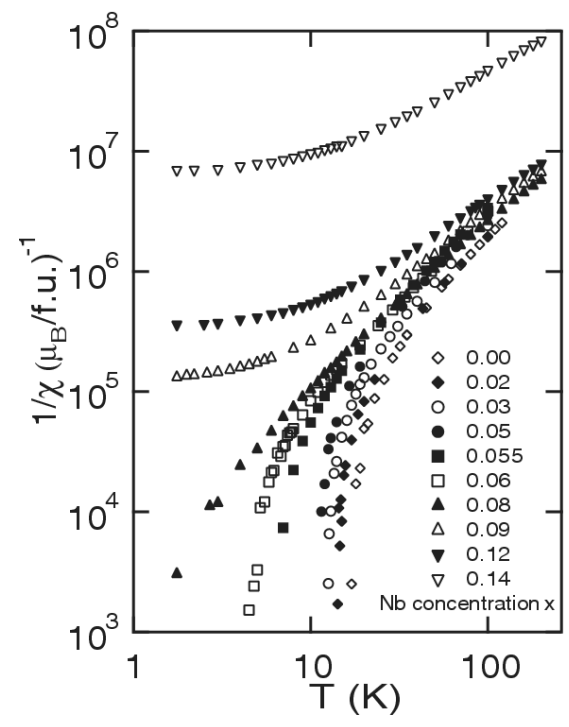

FIG. 25 Temperature dependence of the inverse initial susceptibility $\chi^{-1}$ of $\mathrm{Zr}_{1-x} \mathrm{Nb}_{x} \mathrm{Zn}_{2}$ indicating the critical behavior of $\chi$ near $x \approx 0.08$. From Sokolov et al., 2006.

\section{Superconductivity near the magnetic-non-magnetic quantum phase transition}

For many years, $\mathrm{CeCu}_{2} \mathrm{Si}_{2}$ stood out as the single example of superconductivity in HFS, followed by the discovery of superconductivity in $\mathrm{UPt}_{3}, \mathrm{UBe}_{1} 3$, and $\mathrm{URu}_{2} \mathrm{Si}_{2} . \mathrm{CeCu}_{2} \mathrm{Ge}_{2}$ becomes superconducting above a pressure of $8 \mathrm{GPa}$ (Jaccard, Behnia, and Sierro, 1992; Jaccard et al., 1999). A systematic search by several groups, notably Lonzarich and coworkers, for superconductivity in Ce compounds at the brink of magnetic order led to the discovery of several new heavy-fermion superconductors, $\mathrm{CePd}_{2} \mathrm{Si}_{2}$ (Grosche et al., 1997; Julian et al., 1996, 1998; Mathur et al., 1998), $\mathrm{CeRh}_{2} \mathrm{Si}_{2}$ (Movshovich et al., 1996), $\mathrm{CeIn}_{3}$ (Julian et al., 1996, 1998; Mathur et al., 1998), and possibly $\mathrm{CeCu}_{2}$ (Vargoz, Link, and Jaccard, 1997). In order to observe superconductivity in these systems at the magnetic instability where $T_{\mathrm{N}} \rightarrow 0$, pure samples are often essential. This, together with the very fact that superconductivity appears at a point where low-lying magnetic fluctuations abound, suggest that the superconductivity may be magnetically mediated (Mathur et al., 1998).

Fig. 26 shows the phase diagram for $\mathrm{CePd}_{2} \mathrm{Si}_{2}$ where $T_{\mathrm{N}}$ drops from $10.5 \mathrm{~K}$ at ambient pressure to below $1.6 \mathrm{~K}$ around $25 \mathrm{kbar}$. $T_{\mathrm{N}}$ extrapolates to zero by continuing the linear $T_{\mathrm{N}}(p)$ dependence at $p_{c}=27 \mathrm{kbar}$. Around this critical pressure the superconducting transition temperature $T_{c}$ has its maximum of $\approx 0.6 \mathrm{~K}$ and extends almost symmetrically to $\pm 5 \mathrm{kbar}$ around $p_{c}$. However, it is not clear whether antiferromagnetism gives way to superconductivity just below $p_{c}$ or both types of order coexist.

At $p_{c}$ the electrical resistivity $\rho$ varies with $T^{1.2}$ over almost two orders of magnitude up to $T>30 \mathrm{~K}$ (see inset of Fig. 26). This quasi-linear $T$ dependence of $\rho$, 
together with the linear relationship $T_{\mathrm{N}} \sim\left|p-p_{c}\right|^{\psi}$, $\psi=1$, have led to the suggestion of $2 \mathrm{~d}$ fluctuations (Mathur et al., 1998), as in $\mathrm{CeCu}_{6-x} \mathrm{Au}_{x}$. On the other hand, the anomalous $T^{1.2}$ dependence may arise from an effective crossover of $\rho(T)$ in the $3 \mathrm{~d}$ spin-fluctuation scenario (Rosch, 1999, 2000) as discussed in Sec. III.F. $\mathrm{CeNi}_{2} \mathrm{Ge}_{2}$ exhibits a similar anomalous power law of $\rho(T)$ and a $C / T=\gamma_{0}-\beta \sqrt{T}$ dependence at ambient pressure (Grosche et al., 1997; Julian et al., 1998; Küchler et al., 2003). Some very pure samples even exhibit traces of superconductivity at $p=0$ (Gegenwart et al., 1999). These findings suggest that $\mathrm{CeNi}_{2} \mathrm{Ge}_{2}$ at $p=0$ is right at the magnetic instability, although other samples become superconducting at $p>15 \mathrm{kbar}$ only (Grosche et al., 1997).

It should be mentioned that besides systems exhibiting a narrow dome of superconductivity near a magnetic QCP, exemplified by $\mathrm{CePd}_{2} \mathrm{Si}_{2}$, wide pressure ranges of superconductivity are observed, e.g. in $\mathrm{CeCu}_{2} \mathrm{Ge}_{2}$. Indeed, it has been suggested that superconductivity in $\mathrm{CeCu}_{2} \mathrm{Ge}_{2}$ is mediated by valence fluctuations rather than spin fluctuations which may cause a rather wide range of superconductivity. This idea is corroborated by recent experiments on $\mathrm{CeCu}_{2} \mathrm{Si}_{2}$ doped with $\mathrm{Ge}$ where indeed two disconnected superconducting regions are observed as a function of pressure as shown in Fig. 27 (Yuan et al., 2003, 2006). The relation to quantum criticality is manifest through the pronounced pressure dependence of the residual resistivity $\rho_{0}$ and of the resistivity exponent $\alpha$.

$\mathrm{CeIn}_{3}$ has been suggested to be an antiferromagnet with $3 \mathrm{~d}$ critical fluctuations, giving way to superconduc-

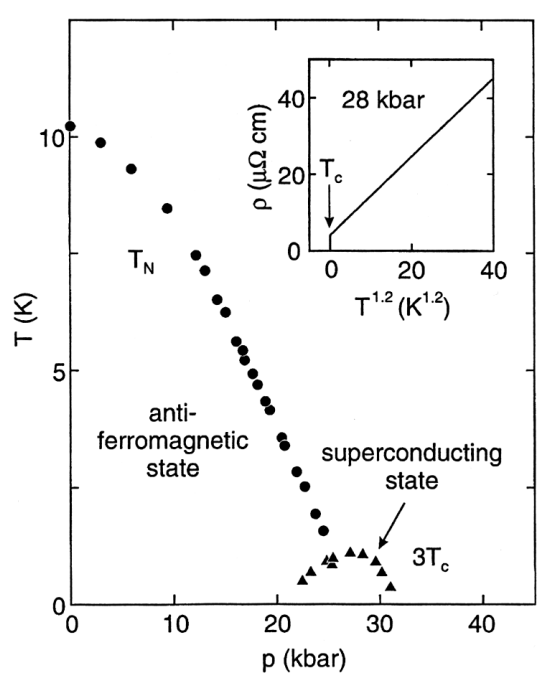

FIG. 26 Temperature-pressure phase diagram of a highpurity $\mathrm{CePd}_{2} \mathrm{Si}_{2}$ single crystal. Superconductivity appears below $T_{c}$ in a narrow window where the Néel temperature $T_{\mathrm{N}}$ tends to absolute zero. For clarity, the values of $T_{c}$ have been scaled by a factor of three, and the origin of the inset has been set below absolute zero. The inset shows that the resistivity $\rho$ exhibits a $T^{1.2}$ dependence on temperature over a wide $T$ range. From Mathur et al., 1998. tivity again below $1 \mathrm{~K}$ under pressure, on account of the $T^{1.5}$ dependence of $\Delta \rho(T)$ close to $p_{c} \approx 25 \mathrm{kbar}$ (Julian et al., 1998; Mathur et al., 1998). In line with this interpretation, the dependence of $T_{\mathrm{N}}$ on $\left|p-p_{c}\right|$ appears to be sub-linear; an exponent $\psi=2 / 3$ is predicted for a 3d antiferromagnet, Eq. (94). However, ${ }^{115}$ In NQR measurements around the critical pressure observed no trace of NFL spin fluctuations, perhaps indicating a first-order magnetic transition (Kawasaki et al., 2004).

The $\mathrm{CeIn}_{3}$-derived CeTIn 5 samples exhibit superconductivity over a wide range in alloying among each other $(T=$ Co, Ir, Rh), as shown in Fig. 28. Particularly interesting is $\mathrm{CeCoIn}_{5}$ (see also Sec. IV.A.4), where a magnetic field induces a second superconducting phase below $B_{c 2}$ (Bianchi et al., 2003a). This phase is a prime candidate for a modulated superconducting state, originally proposed by Fulde and Ferrell (1964) and Larkin and Ovchinnikov (1965), dubbed FFLO state. Last not least, we wish to mention $\mathrm{CeRh}_{2} \mathrm{Si}_{2}$ which becomes superconducting above $p_{c}=6 \mathrm{kbar}$ (Movshovich et al., 1996), however, here antiferromagnetism disappears through a firstorder transition, see Sec. IV.A.2.

A few general remarks are in order. Although the various Ce compounds have vastly different magnetic ordering temperatures, the superconducting $T_{c}$ are quite similar of the order of $0.5 \mathrm{~K}$ with the exception of the "high" $T_{c}=2.3 \mathrm{~K}$ for $\mathrm{CeCoIn}_{5}$. Further, the diverse role of impurities in HFS superconductors is not understood: while some of them are extremely sensitive to impurities, e.g. $\mathrm{CePd}_{2} \mathrm{Si}_{2}$, others are not. In most of the HFS, the question of whether magnetism and superconductivity coexist cooperatively has not been investigated in detail. A prominent counterexample is $\mathrm{CeCu}_{2} \mathrm{Si}_{2}$ where superconductivity competes with A-phase magnetism. We finally note that it has been speculated by Steglich (2005) that

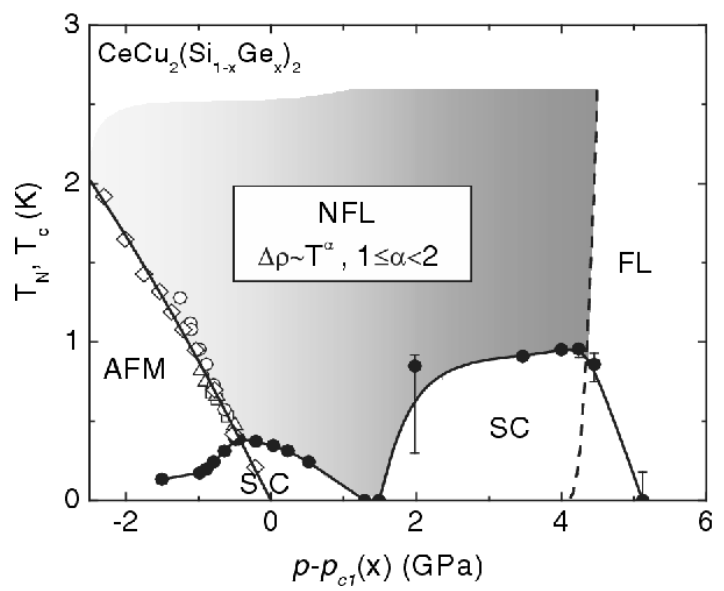

FIG. 27 Combined $(p, x, T)$ diagram of $\mathrm{CeCu}_{2}\left(\mathrm{Si}_{1-x} \mathrm{Ge}_{x}\right)_{2}$. $p_{c 1}(x)$ denotes the pressure-tuned QCP where the Néel temperature $T_{\mathrm{N}} \rightarrow 0 ; p_{c 1}(0)=0, p_{c 1}(2.5)=2.4 \mathrm{GPa}$. Open symbols denote $T_{\mathrm{N}}$, closed symbols the superconducting $T_{c}$. In the wide shaded area, non-Fermi-liquid behavior is observed in the electrical resistivity. From Yuan et al., 2006. 


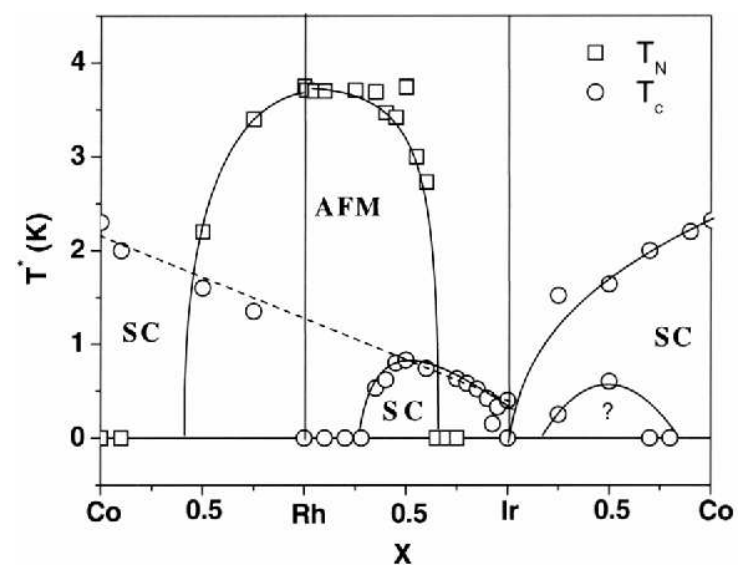

FIG. 28 Phase diagram of $\mathrm{Ce}(\mathrm{Rh}, \mathrm{Ir}, \mathrm{Co}) \mathrm{In}_{5}$ displaying the interplay of antiferromagnetic order (AFM) and superconductivity (SC). From Pagliuso et al., 2002.

conventional LGW critical points favor unconventional superconductivity, while non-LGW QCP disfavor superconductivity in their vicinity.

While all the above systems exhibit superconductivity in the vicinity of antiferromagnetic instabilities, there has been a long-standing quest for superconductivity of an incipient ferromagnet (Fay and Appel, 1980). Initially such type of superconductivity, presumably of spinparallel pairing, was sought among weak itinerant ferromagnets, e.g., $\mathrm{ZrZn}_{2}$. However, the first unambiguous observation of a ferromagnetic superconductor was found in $\mathrm{UGe}_{2}$ under pressure close to the FM instability, with a maximum $T_{c} \simeq 0.4 \mathrm{~K}$ (Saxena et al., 2000), while the related system URhGe becomes superconducting even at ambient pressure $\left(T_{c} \simeq 0.3 \mathrm{~K}\right)$ (Aoki et al., 2001). Fig. 29 shows the pressure-temperature phase diagram of $\mathrm{UGe}_{2}$ as investigated by Pfleiderer and Huxley (2002), revealing two different ferromagnetic phases, overall similar to $\mathrm{ZrZn}_{2}$ (Fig. 24). The different phases are clearly identified by their different ordered moments. As in $\mathrm{ZrZn}_{2}$, the pressure-driven transitions between the FM phases and from ferromagnet to paramagnet are clearly first order. Consequently, the electrical resistivity shows FL-like behavior across the transition, with $\Delta \rho=A T^{2}$ and $A$ peaking at the critical pressure $p_{c}$ (Saxena et al., 2000). The superconductive phase in $\mathrm{UGe}_{2}$ resides completely inside the FM domain, giving strong evidence for spin-parallel pairing in coexistence with ferromagnetism. These observations have instigated numerous theoretical studies (among others Chubukov et al. 2003; Kirkpatrick et al. 2001; Kirkpatrick and Belitz 2003; Roussev and Millis 2001), since the early weak-coupling calculation (Fay and Appel, 1980) suggested two superconductive "domes" just below and above $p_{c}$.

Of particular interest is the observation of two distinct superconductive phases in URhGe as a function of magnetic field (Levy et al., 2005). The low-field phase subsides at a critical field of $B_{c 2}=2 \mathrm{~T}$ applied along the $\mathrm{b}$ axis of the orthorhombic crystal structure. In a field

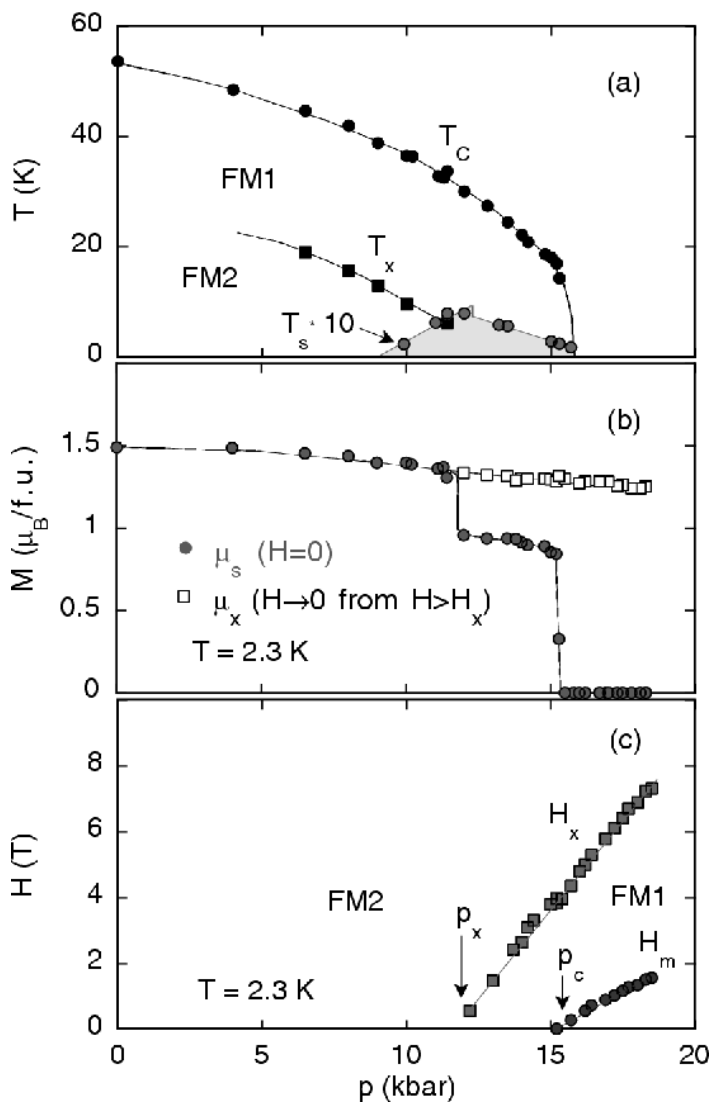

FIG. 29 Phase diagrams of $\mathrm{UGe}_{2}$. (a) Pressure-temperature phase diagram indicating the Curie temperature $T_{\mathrm{C}}$ and the transition temperature $T_{x}$ between two ferromagnetic phases FM1 and FM2. Shaded area is the superconductive transition temperature $T_{S}$. (b) Pressure dependence of the spontaneously ordered moment $M$ in units of $\mu_{B}$ per formula unit at $2.3 \mathrm{~K}$. (c) Pressure-field phase diagram at $T=2.3 \mathrm{~K}$ indicating the metamagnetic transition and the transition between FM1 and FM2. From Pfleiderer and Huxley, 2002.

of $11.7 \mathrm{~T}$ the spin direction changes abruptly. A second superconductive phase appears in the vicinity of this transition, extending between 8 and $13 \mathrm{~T}$. Hence, viewing the spin reorientation as a quantum phase transition, the superconductivity is clearly linked to this QPT.

It has been suggested that the absence of superconductivity in $\mathrm{MnSi}$, even in very pure samples, is due to the lack of inversion symmetry in this material (Saxena et al., 2000). However, superconductivity below $T_{c}=0.75 \mathrm{~K}$ has been observed recently in a HFS lacking inversion symmetry, $\mathrm{CePt}_{3} \mathrm{Si}$, albeit in coexistence with antiferromagnetism, with $T_{c} \approx 2.2 \mathrm{~K}$ (Bauer et al., 2004).

\section{v. CONCLUSIONS}

In general, metallic systems of interacting fermions at low temperature are well described by the Landau Fermiliquid theory. Non-Fermi-liquid behavior over an extended range of temperatures down to absolute zero may 
occur near the borderline between two qualitatively different ground states. The order-parameter fluctuations in the neighborhood of such a quantum critical point can induce singular scattering between the fermions, leading to a breakdown of the usual phase-space arguments on which Fermi-liquid theory is based.

In this review we aimed to give a systematic, balanced and critical account of the present knowledge in the area of Fermi-liquid instabilities near quantum critical points. We focused on magnetic QCP, which are perhaps the best studied cases at present. While a large body of experimental and theoretical work on such systems has been accumulated over the past 20 years, several principal questions appear to be far from answered. Theoretically well established examples of non-Fermi-liquid behavior induced by quantum critical fluctuations are provided by single-site quantum impurity models, a prominent example being the multi-channel Kondo model. Unfortunately, on the experimental side a definite realization of this model in a bulk material is yet to be confirmed.

For lattice systems, the best understood situations are those with only a single variable relevant for the critical behavior, the order parameter. Here, a LandauGinzburg-Wilson field-theoretical description allows one to calculate critical exponents and scaling functions, and a self-consistent RPA-type theory provides in addition approximations for the full dependence of observables on parameters like pressure, temperature, or field, as shown in the pioneering works of Hertz and Moriya, extended by Millis. In general, different universality classes are expected for ferromagnets and antiferromagnets. Further, one should distinguish nominally clean systems, where the critical thermodynamics is not influenced by disorder, from disordered ones. (The latter case is more complicated, as disorder may modify critical exponents or even destroy the zero-temperature phase transition.) Experimentally well characterized examples of systems following the LGW predictions for clean systems appear to be $\mathrm{CeNi}_{2} \mathrm{Ge}_{2}$ and $\mathrm{Ce}_{1-x} \mathrm{La}_{x} \mathrm{Ru}_{2} \mathrm{Si}_{2}$, which are close to an antiferromagnetic instability.

Somewhat unexpectedly, a growing number of systems has been discovered showing properties inconsistent with those of LGW theory. A common theme is that further soft variables exist such that the LGW approach is no longer applicable. These soft variables may be either the fermionic particle-hole excitations which strongly couple to the order parameter (as is the case for ferromagnets), or additional degrees of freedom like those associated with the Kondo effect in heavy-fermion systems. A full understanding of these more complex situations of coupled slow modes is not available at present, although several interesting proposals exist and have been reviewed above.

In the following we try to summarize what we think are pressing questions in the field, starting with the theory side. (T1) Can one formulate a theory for the critical behavior in metallic magnets for the approach from the ordered side, along the works of Hertz and
Moriya? (T2) Can one analyze a coupled theory of orderparameter fluctuations and fermions for clean metallic magnets using RG techniques? (T3) Can one prove that the LGW theory for $3 \mathrm{~d}$ antiferromagnets is stable and self-consistent? Is there room for non-LGW criticality in 3d antiferromagnets? (T4) What are the characteristics of the metallic antiferromagnetic QPT in $d=2$ ? (T5) Can one develop one of the scenarios of the breakdown of the Kondo effect (Sec. III.I) to consistently describe the phenomenology of materials like $\mathrm{CeCu}_{6-x} \mathrm{Au}_{x}$ or $\mathrm{YbRh}_{2} \mathrm{Si}_{2}$ ? (T6) Can one understand in more detail the physics of QPT smeared by disorder? (T7) Are there scenarios for stable NFL phases in $3 \mathrm{~d}$ systems, which may explain, e.g., the physics of $\mathrm{MnSi}$ ?

Important directions for experimental work are: (E1) A detailed analysis of energy- and momentum-resolved magnetic fluctuations of materials with non-LGW criticality (in addition to $\mathrm{CeCu}_{6-x} \mathrm{Au}_{x}$ ) would be instructive. Is there $\omega / T$ scaling? Are quasi-2d fluctuations generic? (E2) Given a nominally clean material with a wellcharacterized QCP, it would be important to systematically study the influence of disorder on the critical properties. (E3) Can one find a "dirty ferromagnet" which follows the predictions of the theory in Sec. III.H.1 (which is the only well-developed theory of order-parameter fluctuations coupled to fermionic modes)? (E4) Can one single out heavy-fermion systems showing clear-cut evidence for a jump in the Fermi volume as $T \rightarrow 0$ ? (E5) Can one identify two or more distinct diverging time/length scales near certain magnetic heavy-fermion critical points? (E6) Can one establish a link between the presence or absence of superconductivity near a QCP and the universality class of the QCP?

\section{Acknowledgments}

It is our pleasure to acknowledge illuminating conversations and collaborations with, among others, D. Belitz, A. V. Chubukov, P. Coleman, J. Flouquet, M. Garst, P. Gegenwart, G. G. Lonzarich, M. B. Maple, S. Paschen, C. Pfleiderer, L. Pintschovius, S. Sachdev, J. Schmalian, T. Senthil, Q. Si, F. Steglich, O. Stockert, and T. Vojta. We thank L. Behrens, Y. Kodak, B. Schelske, R. Schrempp, C. Vojta, and M. Uhlarz for technical support. This research was supported by the DFG through SFB 608 and GRK 284, by the Helmholtz Virtual Institute for Quantum Phase Transitions (Karlsruhe), and by NSF Grant PHY99-0794 (KITP Santa Barbara).

\section{References}

Abanov, A., A. V. Chubukov, and A. M. Finkelstein, Europhys. Lett. 54, 488 (2001).

Abanov, A., and A. V. Chubukov, 2004, Phys. Rev. Lett. 93, 255702 .

Affleck, I., and A. W. W. Ludwig, 1991a, Nucl. Phys. B 352, 849. 
Affleck, I., and A. W. W. Ludwig, 1991b, Nucl. Phys. B 360, 641.

Akhiezer, I. A., and E. M. Chudnovskii, 1976, Fix. Tverd. Tela (Leningrad) 18, 1427 [Sov. Phys. Solid State, 1976, 18, 827].

Aleiner, I. L., and K. B. Efetov, 2006, Phys. Rev. B 74, 075102.

Altshuler, B. L., and A. G. Ahronov, in Electron Electron Interaction in Disordered Systems, edited by A. L. Efros and M. Pollack (North Holland, Amsterdam 1985).

Altshuler, B. L., L. B. Ioffe, and A. J. Millis, 1995, Phys. Rev. B 52, 5563.

Amato, A., D. Jaccard, J. Flouquet, F. Lapierre, J. L. Tholence, R. A. Fisher, S. E. Lacy, J. A. Olsen, and N. E. Phillips, 1987, J. Low Temp. Phys. 68, 371.

Amato, A., R. Feyerherm, F. N. Gygax, D. Jaccard, A. Schenck, J. Sierro, E. Walker, and U. Zimmermann, 1993, Physica B 186-188, 273.

Amato, A., R. Feyerherm, F. N. Gygax, A. Schenck, J. Flouquet, and P. Lejay, 1994, Phys. Rev. B 50, 619.

Amato, A., R. Feyerherm, F. N. Gygax, A. Schenck, H. v. Löhneysen, and H. G. Schlager, 1995, Phys. Rev. B 52, 54.

Anderson, P. W., 1961, Phys. Rev. 124, 41.

Anderson, P. W., 1970, J. Phys. C 3, 2436.

Anderson, P. W., and G. Yuval, 1970, Phys. Rev. B 1, 1522.

Anderson, P. W., The Theory of Superconductivity in the High- $T_{c}$ Cuprates, (Princeton University Press, Princeton, New Jersey 1997).

Andraka, B., and A. M. Tsvelik, 1991, Phys. Rev. Lett. 67, 2886.

Andrei, N., 1980, Phys. Rev. Lett. 45, 379.

Andrei, N., K. Furuya, and J. H. Lowenstein, 1983, Rev. Mod. Phys. 55, 331.

Andrei, N., and C. Destri, 1984, Phys. Rev. Lett. 52, 364.

Andres, K., J. E. Graebner, and H. R. Ott, 1975, Phys. Rev. Lett. 35, 1779.

Aoki, D., A. Huxley, E. Ressouche, D. Braithwaite, J. Flouquet, P. Brison, E. Lhotel, and C. Paulsen, 2001, Nature 413, 613 .

Aronson, M. C., R. Osborn, R. A. Robinson, J. W. Lynn, R. Chau, C. L. Seaman, and M. B. Maple, 1995, Phys. Rev. Lett. 75, 725 .

Bak, P., and M. H. Jensen, 1980, J. Phys. C 13, L881.

Bao, W., G. Aeppli, J. W. Lynn, P. G. Pagliuso, J. L. Sarrao, M. F. Hundley, J. D. Thompson, and Z. Fisk, 2002, Phys. Rev. B 65, 100505.

Bauer, E. D., G. Hilscher, H. Michor, C. Paul, E. W. Scheidt, A. Gribanov, Yu. Seropegin, H. Noel, M. Sigrist, and P. Rogl, 2004, Phys. Rev. Lett. 92, 027003.

Bauer, E. D., C. Capan, F. Ronning, R. Movshovich, J. D. Thompson, and J. L. Sarrao, 2005, Phys. Rev. Lett. 94, 047001.

Baym, G., and C. Pethick, 1991, Landau Fermi-Liquid Theory, Wiley, New York.

Belitz, D., T. R. Kirkpatrick, and T. Vojta, 1997, Phys. Rev. B 55, 9452.

Belitz, D., T. R. Kirkpatrick, and T. Vojta, 1999, Phys. Rev. Lett. 82, 4707.

Belitz, D., T. R. Kirkpatrick, R. Narayanan, and T. Vojta, 2000, Phys. Rev. Lett. 85, 4602.

Belitz, D., T. R. Kirkpatrick, M. T. Mercaldo, S. L. Sessions, 2001, Phys. Rev. B 63, 174427 and 174428.

Belitz, D., T. R. Kirkpatrick, and J. Rollbühler, 2004, Phys. Rev. Lett. 93, 155701.
Belitz, D., T. R. Kirkpatrick, and J. Rollbühler, 2005, Phys. Rev. Lett. 94, 247205.

Belitz, D., T. R. Kirkpatrick, and A. Rosch, 2006, Phys. Rev. B 73, 054431.

Belitz, D., T. R. Kirkpatrick, and A. Rosch, 2006, Phys. Rev. B 74, 024409.

Bernal, O. O., D. E. McLaughlin, H. G. Lukefahr, and B. Andraka, 1995, Phys. Rev. Lett. 75, 2023.

Bernal, O. O., D. E. MacLaughlin, A. Amato, R. Feyerherm, F. N. Gygax, A. Schenck, R. H. Heffner, L. P. Lee, G. J. Nieuwenhuys, B. Andraka, H. v. Löhneysen, O. Stockert, and H. R. Ott, 1996, Phys. Rev. B 54, 13000.

Bianchi, A., R. Movshovich, C. Capan, P. G. Pagliuso, and J. L. Sarrao, 2003, Phys. Rev. Lett. 91, 187004.

Bianchi, A., R. Movshovich, I. Vekhter, P. G. Pagliuso, and J. L. Sarrao, 2003, Phys. Rev. Lett. 91, 257001.

Binz, B., A. Vishwanath, and V. Aji, 2006, Phys. Rev. Lett. 96, 207202.

Block, A., M. Abd-Elmeguid, and H. Micklitz, 1994, Phys. Rev. B 49, 12365.

Böhm, A., R. Caspary, U. Habel, L. Pawlak, A. Zuber, F. Steglich, and A. Loidl, 1988, J. Mag. Mag. Mater. 76-77, 150 .

Bogenberger, B., and H. v. Löhneysen, 1995, Phys. Rev. Lett. 74, 1016.

Booth, C. H., E.-W. Scheidt, U. Killer, A. Weber, and S. Kehrein, 2002, Phys. Rev B 66, 140402.

Bruls, G., B. Wolf, D. Finsterbusch, P. Thalmeier, I. Kouroudis, W. Sun, W. Assmus, B. Lüthi, M. Lang, K. Gloos, F. Steglich, and R. Modler, 1994, Phys. Rev. Lett. 72, 1754.

Bulla, R., 1999, Phys. Rev. Lett. 83, 136.

Burdin, S., A. Georges, and D. R. Grempel, 2000, Phys. Rev. Lett. 85, 1048.

Calemczuk, R., E. Bonjour, J. Rossat-Mignod, and B. Chevalier, 1990, J. Mag. Mag. Mater. 90-91, 477.

Capan, C., A. Bianchi, F. Ronning, A. Lacerda, J. D. Thompson, M. F. Hundley, P. G. Pagliuso, J. L. Sarrao, and R. Movshovich, 2004, Phys. Rev. B 70, 180502(R).

Castro Neto, A. H., G. Castilla, and B. A. Jones, 1998, Phys. Rev. Lett. 81, 3531.

Castro Neto, A. H., and B. A. Jones, 2000, Phys. Rev. B 62, 14975.

Chattopadhyay, T., H. v. Löhneysen, T. Trappmann, and M. Loewenhaupt, 1990, Z. Phys. B 80, 159.

Chayes, J. T., L. Chayes, D. S. Fisher, and T. Spencer, 1986, Phys. Rev. Lett. 57, 2999.

Chubukov, A. V., A. M. Finkel'stein, R. Haslinger, and D. K. Morr, 2003, Phys. Rev. Lett. 90, 077002 .

Chubukov, A. V., and D. L. Maslov, 2003, Phys. Rev. B 68, 155113.

Chubukov, A. V., and D. L. Maslov, 2004, Phys. Rev. B 69, 121102.

Chubukov, A. V., C. Pépin, and J. Rech, 2004, Phys. Rev. Lett. 92, 147003.

Chubukov, A. V., D. L. Maslov, S. Gangadharaiah, and L. I. Glazman, 2005, Phys. Rev. B 71, 205112.

Chubukov, A. V., D. L. Maslov, and A. J. Millis, 2006, Phys. Rev. B 73, 045128 .

Coleman, P., 1999, Physica B 259-261, 353.

Coleman, P., C. Pépin, Q. Si, and R. Ramazashvili, 2001, J. Phys. Cond. Matt. 13, R723.

Coleman, P., J. B. Marston, and A. J. Schofield, 2005, Phys. Rev. B 72, 245111. 
Costi, T. A., and A. C. Hewson, 1990, Physica B 163, 179.

Costi, T. A., and A. C. Hewson, 1992, Phil. Mag. B 65, 1165.

Costi, T. A., J. Kroha, and P. Wölfle, 1996, Phys. Rev. B 53, 1850.

Cox, D. L., and A. Zawadowski, 1998, Adv. Phys. 47, 599.

Custers, J., P. Gegenwart, H. Wilhelm, K. Neumaier, Y. Tokiwa, O. Trovarelli, C. Geibel, F. Steglich, C. Pépin, and P. Coleman, 2003, Nature 424, 524.

Dalidovich, D., and V. Dobrosavljevic, 2002, Phys. Rev. B 66, 081107.

de Andrade, M. C., R. Chau, R. P. Dickey, N. R. Dilley, E. J. Freeman, D. A. Gajewski, M. B. Maple, R. Movshovich, A. H. Castro Neto, G. Castilla, and B. A. Jones, 1998, Phys. Rev. Lett. 81, 5620.

de Boer, F. R., J. C. P. Klaasse, P. A. Veenhuizen, A. Böhm, C. D. Bredl, U. Gottwick, H. M. Mayer, L. Pawlak, U. Rauchschwalbe, H. Spille, and F. Steglich, 1987, J. Mag. Mag. Mater. 63-64, 91.

Dell'Anna, L., and W. Metzner, 2006, Phys. Rev. B 73, 045127.

Djerbi, R., P. Haen, F. Lapierre, P. Lehmann, and J. P. Kappler, 1988, J. Mag. Mag. Mater. 76-77, 260.

Dobrosavljević, V., and E. Miranda, 2005, Phys. Rev. Lett. 94, 187203.

Doiron-Leyraud, N., I. R. Walker, L. Taillefer, M. J. Steiner, S. R. Julian, and G. G. Lonzarich, 2003, Nature 425, 595.

Doniach, S., 1977, Physica B 91, 231.

Estrela, P., A. de Visser, T. Naka, F. R. de Boer, and L. C. J. Pereira, 2001, Eur. Phys. J. B 23, 449.

Estrela, P., A. de Visser, F. R. de Boer, T. Naka, and L. Shlyk, 2001, Phys. Rev. B 63, 212409.

Fawcett, E., J. P. Maita, and J. H. Wernick, 1970, Int. J. Magn. 1, 29.

Fay, D., and J. Appel, 1980, Phys. Rev. B 22, 3173.

Feldman, J., J. Magnen, V. Rivasseu, and E. Trubowitz, Europhys. Lett., 1993, 24, 437.

Fert, A., and P. M. Levy, 1987, Phys. Rev. B 36, 1907.

Ferstl, J., C. Geibel, F. Weickert, P. Gegenwart, T. Radu, T. Lühmann, and F. Steglich, 2005, Physica B 359-361, 26.

Finsterbusch, D., H. Willig, B. Wolf, G. Bruls, B. Lüthi, M. Waffenschmidt, O. Stockert, A. Schröder, and H. von Löhneysen, 1996, Ann. Physik (Leipzig) 5, 184.

Fischer, I., N. Shah, and A. Rosch, 2007, e-print arXiv:condmat/0702287.

Fischer, I., and A. Rosch, 2004, Europhys. Lett. 68, 93.

Fischer, I., and A. Rosch, 2005, Phys. Rev. B 71, 184429.

Fisher, R. A., C. Marcenat, N. E. Phillips, P. Haen, F. Lapierre, P. Lejay, J. Flouquet, and J. Voiron, 1991, J. Low Temp. Phys. 84, 49.

Fisher, D. S., 1995, Phys. Rev. B 51, 6411.

Fisher, R. A., F. Bouquet, N. E. Phillips, M. F. Hundley, P. G. Pagliuso, J. L. Sarrao, Z. Fisk, and J. D. Thompson, 2002, Phys. Rev. B 65, 224509.

Fisk, Z., H. R. Ott, and G. Aeppli, 1987, Jpn. J. Appl. Phys. 26, Suppl. 26-3, 1882.

Flouquet, J., S. Kambe, L. P. Regnault, P. Haen, J. P. Brison, F. Lapierre, and P. Lejay, 1995, Physica B 215, 77.

Fontes, M. B., M. A. Continentino, S. L. Bud'ko, M. ElMassalami, L. C. Sampaio, A. P. Guimaraes, E. BaggioSaitovitch, M. F. Hundley, and A. Lacerda, 1996, Phys. Rev. B 53, 11678.

Fraunberger, G., B. Andraka, J. S. Kim, U. Ahlheim, and G. R. Stewart, 1989, Phys. Rev. B 40, 4735.

Freltoft, T., P. Böni, G. Shirane, and K. Motoya, 1988, Phys.
Rev. B 37, 3454 .

Fulde, P., and R. A. Ferrell, 1964, Phys. Rev. 135, A550.

Gajewski, D. A., R. Chau, and M. B. Maple, 2000, Phys. Rev. B 62, 5496.

Gangopadhyay, A. K., J. S. Schilling, E. Schuberth, P. Gutsmiedl, F. Gross, and K. Andres, 1988, Phys. Rev. B 38, 2603.

Garst, M., 2003, PhD thesis, University of Karlsruhe.

Gavilano, J. L., J. Hunziker, and H. R. Ott, 1995, Phys. Rev. B 52, R13106.

Gegenwart, P., C. Langhammer, C. Geibel, R. Helfrich, M. Lang, G. Sparn, F. Steglich, R. Horn, L. Donnevert, A. Link, and W. Assmus, 1998, Phys. Rev. Lett. 81, 001501.

Gegenwart, P., F. Kromer, M. Lang, G. Sparn, C. Geibel, and F. Steglich, 1999, Phys. Rev. Lett. 82, 1293.

Gegenwart, P., J. Custers, C. Geibel, K. Neumaier, T. Tayama, K. Tenya, O. Trovarelli, and F. Steglich, 2002, Phys. Rev. Lett. 89, 056402.

Gegenwart, P., J. Custers, Y. Tokiwa, C. Geibel, and F. Steglich, 2005, Phys. Rev. Lett. 94, 076402.

Gegenwart, P., T. Westerkamp, C. Krellner, Y. Tokiwa, S. Paschen, C. Geibel, F. Steglich, E. Abrahams, and Q. Si, 2007, Science 315, 969.

Germann, A., A. K. Nigam, J. Dutzi, A. Schröder, and H. v. Löhneysen, 1988, J. Physique Coll. 49, C8 755.

Germann, A., and H. v. Löhneysen, 1989, Europhys. Lett. 9, 367.

Georges, A., G. Kotliar, W. Krauth, and M. J. Rozenberg, 1996, Rev. Mod. Phys. 68, 13.

Graf, T., J. D. Thompson, M. F. Hundley, R. Movshovich, Z. Fisk, D. Mandrus, R. A. Fisher, and N. E. Phillips, 1997, Phys. Rev. Lett. 78, 3769.

Gratz, E., E. Bauer, H. Nowotny, H. Mueller, S. Zemirli, and B. Barbara, 1987, J. Mag. Mag. Mater. 63-64, 312.

Grempel, D. R., and Q. Si, 2003, Phys. Rev. Lett. 91, 026401.

Grewe, N., and F. Steglich, in: Handbook of Physics and Chemistry of Rare Earths Vol. 14 (K. A. Gschneidner Jr., and L. Eyring, eds.), Elsevier Amsterdam (1991), p. 343.

Greywall, D. S., 1983, Phys. Rev. B 27, 2747.

Grier, B. H., J. M. Lawrence, V. Murgai, and R. D. Parks, 1984, Phys. Rev. B 29, 2664.

Griffiths, R. B., 1969, Phys. Rev. Lett. 23, 17.

Grigera, S. A., R. S. Perry, A. J. Schofield, M. Chiao, S. R. Julian, G. G. Lonzarich, S. I. Ikeda, Y. Maeno, A. J. Millis, and A. P. Mackenzie, 2001, Science 294, 329.

Grosche, F. M., S. J. S. Lister, F. V. Carter, S. S. Saxena, R. K. W. Haselwimmer, N. D. Mathur, S. R. Julian, and G. G. Lonzarich, 1997, Physica B 239, 62.

Grosche, F. M., P. Agarwal, S. R. Julian, N. J. Wilson, R. K. W. Haselwimmer, S. J. S. Lister, N. D. Mathur, F. V. Carter, S. S. Saxena, and G. G. Lonzarich, 2000, J. Phys. Cond. Matter 12, L533.

Grote, I., E. Körding, and F. Wegner, 2002, J. Low Temp. Phys. 126 , 1385.

Grube, K., W. H. Fietz, U. Tutsch, O. Stockert, and H. v. Löhneysen, 1999, Phys. Rev. B. 60, 11947.

Gu, H. W., J. Tang, A. Matsushita, T. Taniguchi, Y. Tabata, and Y. Miyako, 2002, Physica B 312-313, 248.

Halboth, C. J., and W. Metzner, 2000, Phys. Rev. Lett. 85, 5162.

Haen, P., J. Flouquet, F. Lapierre, P. Lejay, and G. Remenyi, 1987, J. Low Temp. Phys. 67, 391.

Harris, A. B., 1974, J. Phys. C 7, 1671.

Hasegawa, H., and T. Moriya, 1974, J. Phys. Soc. Jpn. 36, 
1542.

Hauser, R., M. Galli, E. Bauer, A. Kottar, G. Hilscher, and D. Kaczorowski, 1998, J. Mag. Mag. Mater. 177-181, 292.

Hegger, H., C. Petrovic, E. G. Moshopoulou, M. F. Hundley, J. L. Sarrao, Z. Fisk, and J. D. Thompson, 2000, Phys. Rev. Lett. 84, 4986.

Hertz, J. A., 1976, Phys. Rev. B 14, 1165.

Heuser, K., E.-W. Scheidt, T. Schreiner, and G. R. Stewart, 1998a, Phys. Rev. B 57, R4198.

Heuser, K., E.-W. Scheidt, T. Schreiner, and G. R. Stewart, 1998b, Phys. Rev. B 58, R15959.

Hewson, A. C., 1993, The Kondo Problem to Heavy Fermions, Cambridge University Press, Cambridge.

Hlubina, R., and T. M. Rice, 1995, Phys. Rev. B 51, 9253.

Holstein, T., R. E. Norton, and P. Pincus, 1973, Phys. Rev. B 8, 2649.

Holtmeier, S., P. Haen, A. Lacerda, P. Lejay, J. L. Tholence, J. Voiron, and J. Flouquet, 1995, Physica B 204, 250 and refs. therein.

Houghton A., N. Read, and H. Won, 1988, Phys. Rev. B 37, 3782.

Huber, J. G., M. B. Maple, D. Wohlleben, and G. S. Knapp, 1975, Solid State Commun. 16, 211.

Iglesias, J. R., C. Lacroix, and B. Coqblin, 1997, Phys. Rev. B 56, 11820.

Ioffe, L. B. and A. J. Millis, 1995, Phys. Rev. B 51, 16151.

Ishida, K., K. Okamoto, Y. Kawasaki, Y. Kitaoka, O. Trovarelli, C. Geibel, and F. Steglich, 2002, Phys. Rev. Lett. 89, 107202.

Ishikawa, Y., Y. Noda, Y. J. Uemura, C. F. Majkrzak, and G. Shirane, 1985, Phys. Rev. B 31, 5884.

Jaccard, D., K. Behnia, and J. Sierro, 1992, Phys. Lett. A 163, 475 .

Jaccard, D., H. Wilhelm, K. Alami-Yadri, and E. Vargoz, 1999, Physica B 259-261, 1.

Jeffries, J. R., N. A. Frederick, E. D. Bauer, H. Kimura, V. S. Zapf, K.-D. Hof, T. A. Sayles, and M. B. Maple, 2005, Phys. Rev. B 72, 024551.

Julian, S. R., C. Pfleiderer, F. M. Grosche, N. D. Mathur, G. J. McMullan, A. J. Diver, I. R. Walker, and G. G. Lonzarich, 1996, J. Phys. Cond. Matter 8, 9675.

Jarrell, M., 1995, Phys. Rev. B 51, 7429.

Julian, S. R., F. V. Carter, F. M. Grosche, R. K. W. Haselwimmer, S. J. Lister, N. D. Mathur, G. J. McMullan, C. Pfleiderer, S. S. Saxena, I. R. Walker, N. J. W. Wilson, and G. G. Lonzarich, 1998, J. Mag. Mag. Mater. 177-181, 265.

Kadowaki, K., and S. B. Woods, 1986, Solid State Commun. $\mathbf{5 8 ,} 507$.

Kadowaki, H., M. Sato, and S. Kawarazaki, 2004, Phys. Rev. Lett. 92, 097204.

Kadowaki, H., Y. Tabata, M. Sato, N. Aso, S. Raymond, and S. Kawarazaki, 2006, Phys. Rev. Lett. 96, 016401.

Kambe, S., S. Raymond, L.-P. Regnault, J. Flouquet, P. Lejay, and P. Haen, 1996, J. Phys. Soc. Jpn. 65, 3294.

Kawarazaki, S., Y. Kobashi, J. A. Fernandez-Baca, S. Murayama, Y. Ōnuki, and Y. Miyako, 1995, Physica B 206 207, 298.

Kawasaki, S., et al., 2004, J. Phys. Soc Jpn 73, 1647.

Kee, H.-Y., E. H. Kim, and C.-H. Chung, 2003, Phys. Rev. B 68, 245109.

Khavkine, I., C.-H. Chung, V. Oganesyan, and H.-Y. Kee, 2004, Phys. Rev. B 70, 155110.

Kirkpatrick, T. R., and D. Belitz, 1996, Phys. Rev. Lett. 76, 2571.
Kirkpatrick, T. R., and D. Belitz, 1996, Phys. Rev. B 53, 14364.

Kirkpatrick, T. R., D. Belitz, T. Vojta, and R. Narayanan, 2001, Phys. Rev. Lett. 87, 127003.

Kirkpatrick, T. R., and D. Belitz, 2003, Phys. Rev. B 67, 024515.

Kimura, N., M. Endo, T. Isshiki, S. Minagawa, A. Ochiai, H. Aoki, T. Terashima, S. Uji, T. Matsumoto, and G. G. Lonzarich, 2004, Phys. Rev. Lett. 92, 197002.

Kiselev, M., K. Kikoin, and R. Oppermann, 2002, Phys. Rev. B 65, 184410.

Kivelson, S. A., E. Fradkin, and V. J. Emery, 1998, Nature 393, 550 .

Knafo, W., S. Raymond, J. Flouquet, B. Fåk, M. A. Adams, P. Haen, F. Lapierre, S. Yates, and P. Lejay, 2004, Phys. Rev. B 70, 174401.

Knebel, G., D. Aoki, D. Braithwaite, B. Salce, and J. Flouquet, 2006, Phys. Rev. B 74, 020501.

Kondo, J., 1964, Prog. Theor. Phys. 32, 37.

Kontani, H., K. Kanki, and K. Ueda, 1999, Phys. Rev. B 59 14723.

Krishna-murthy, H. R., J. W. Wilkins, and K. G. Wilson, 1980, Phys. Rev. B 21, 1003.

Kroha, J., and P. Wölfle, 2005, J. Phys. Soc. Jpn. 74, 16.

Küchler, R., N. Oeschler, P. Gegenwart, T. Cichorek, K. Neumaier, O. Tegus, C. Geibel, J. A. Mydosh, F. Steglich, L. Zhu, and Q. Si, 2003, Phys. Rev. Lett. 91, 066405.

Küchler, R., P. Gegenwart, K. Heuser, E.-W. Scheidt, G. R. Stewart, and F. Steglich, 2004, Phys. Rev. Lett. 93, 096402.

Lakner, M., H. v. Löhneysen, A. Langenfeld, and P. Wölfle, 1994, Phys. Rev. B 50, 17064.

Landau, L. D., 1957, Sov. Phys. JETP 3, 920.

Landau, L. D., 1957, Sov. Phys. JETP 5, 101.

Landau, L. D., 1959, Sov. Phys. JETP 8, 70.

Lapertot, G., R. Calemczuk, C. Marcenat, J. Y. Henry, J. X. Boucherle, J. Flouquet, J. Hammann, R. Cibin, J. Cors, D. Jaccard, and J. Sierro, 1993, Physica B 186-188, 454.

Larkin, A. I., and Y. N. Ovchinnikov, 1965, Sov. Phys. JETP 20, 762 .

Lee, P. A., and T. V. Ramakrishnan, 1985, Rev. Mod. Phys. $\mathbf{5 7}, 287$.

Lee, M., A. Husmann, T. F. Rosenbaum, and G. Aeppli, 2004, Phys. Rev. Lett. 92, 187201.

Leggett, A. J., S. Chakravarty, A. T. Dorsey, M. P. A. Fisher, A. Garg, and W. Zwerger, 1987, Rev. Mod. Phys. 59, 1.

Levy, F., I. Sheikin, B. Grenier, and A. D. Huxley, 2005, Science 309, 1343.

Lin, C. L., A. Wallash, J. E. Crow, T. Mihalisin, and P. Schlottmann, 1987, Phys. Rev. Lett. 58, 1232.

Lloret, B., B. Chevalier, B. Buffat, J. Etourneau, S. Quezel, A. Lamharrar, J. Rossat-Mignod, R. Calemczuk, and E. Bonjour, 1987, J. Mag. Mag. Mater. 63-64, 85.

Lonzarich, G. G., and L. Taillefer, 1985, J. Phys. C. 18, 4339. Ma, S.-k., C. Dasgupta, and C.-k. Hu, 1979, Phys. Rev. Lett. 43, 1434.

MacLaughlin, D. E., M. S. Rose, J. E. Anderson, O. O. Bernal, R. H. Heffner, G. J. Nieuwenhuys, R. E. Baumbach, N. P. Butch, and M. B. Maple, 2006, Physica B 374, 177.

Maebashi, H., K. Miyake, and C. M. Varma, 2005, Phys. Rev. Lett. 95, 207207.

Mathur, N. D., F. M. Grosche, S. R. Julian, I. R. Walker, D. M. Freye, R. K. W. Haselwimmer, and G. G. Lonzarich, 1998, Nature 394, 39. 
Mathon, J, 1968, Proc. Roy. Soc. A 306, 355.

McCoy, B. M., and T. T. Wu, 1968, Phys. Rev. 176, 631.

McMahan A. K., C. Huscroft, R. T. Scalettar, and E. L. Pollock, 1998, J. of Comp.-Aided Mat. Design 5, 131.

Mena, F. P., D. van der Marel, A. Damascelli, M. Fäth, A. A. Menovsky, and J. A. Mydosh, 2003, Phys. Rev. B 67, 241101(R)

Metzner, W., and D. Vollhardt, 1989, Phys. Rev. Lett. 62, 324.

Metzner, W., D. Rohe, and S. Andergassen, 2003, Phys. Rev. Lett. 91, 066402.

Millis, A. J., 1993, Phys. Rev. B 48, 7183.

Millis, A. J., A. J. Schofield, G. G. Lonzarich, and S. A. Grigera, 2002, Phys. Rev. Lett. 88, 217204.

Millis, A. J., D. K. Morr, and J. Schmalian, 2002, Phys. Rev. B 66, 174433.

Miranda, E., and V. Dobrosavljević, 2001, Phys. Rev. Lett. 86, 264.

Miranda, E., and V. Dobrosavljević, 2005, Rep. Prog. Phys. 68, 2337.

Miyako, Y., T. Takeuchi, T. Taniguchi, Y. Yamamoto, S. Kawarazaki, M. Acet, G. Dumpich, and E. F. Wassermann, 1996, Z. Phys. B 101, 339.

Miyako, Y., S. Kawarazaki, T. Taniguchi, T. Takeuchi, K. Marumoto, R. Hamada, Y. Yamamoto, M. Sato, Y. Tabata, H. Tanabe, M. Ocio, P. Pari, and J. Hammann, 1997, Physica B 230-232, 1011.

Mock, S., C. Paschke, A. Schröder, J. Sereni, M. Sieck, and H. v. Löhneysen, 1994, Physica B 199-200, 39.

Monthoux, P., and G. G. Lonzarich, 1999, Phys. Rev. B 59, 14598.

Moriya, T., 1985, Spin Fluctuations in Itinerant Electron Magnetism (Springer, Berlin).

Moriya, T., and A. Kawabata, 1973, J. Phys. Soc. Jpn. 34, 639.

Moriya, T., and T. Takimoto, 1995, J. Phys. Soc. Jpn. 64, 960.

Moriya, T., 2006, Proc. Jpn. Acad. Ser. B 82, 1.

Motrunich, O., S.-C. Mau, D. A. Huse, and D. S. Fisher, 2000, Phys. Rev. B 61, 1160.

Movshovich, R., T. Graf, D. Mandrus, M. F. Hundley, J. D. Thompson, R. A. Fisher, N. E. Phillips, and J. L. Smith, 1996, Physica B 223-224, 126.

Murayama, S., C. Sekine, A. Yokoyanagi, K. Hoshi, and Y. Ōnuki, 1997, Phys. Rev. B 56, 11092.

Muramatsu, T., N. Tateiwa, T. C. Kobayashi, K. Shimizu, K. Amaya, D. Aoki, H. Shishido, Y. Haga, and Y. Onuki, 2001, J. Phys. Soc. Jpn. 70, 3362.

Mydosh, J., 1993, Spin Glasses: An Experimental Introduction, Taylor and Francis, London.

Nakanishi, O., A. Yanase, A. Hasegawa, and M. Kataoka, 1980, Solid State Commun. 35, 995.

Nakatsuji, S., S. Yeo, L. Balicas, Z. Fisk, P. Schlottmann, P. G. Pagliuso, N. O. Moreno, J. L. Sarrao, and J. D. Thompson, 2002, Phys. Rev. Lett. 89, 106402.

Narayanan, R., T. Vojta, D. Belitz, and T. R. Kirkpatrick, 1999, Phys. Rev. Lett. 82, 5132.

Neumayr, A., and W. Metzner, 2003, Phys. Rev. B 67, 035112.

Newns, D. M., and N. Read, 1987, Adv. Phys. 36, 799.

Nicklas, M., M. Brando, G. Knebel, F. Mayr, W. Trinkl, and A. Loidl, 1999, Phys. Rev. Lett. 82, 4268.

Nicklas, M., R. Borth, E. Lengyel, P. G. Pagliuso, J. L. Sarrao, V. A. Sidorov, G. Sparn, F. Steglich, and J. D. Thompson,
2001, J. Phys. Cond. Matter 13, L905.

Niklowitz, P. G., F. Beckers, G. G. Lonzarich, G. Knebel, B. Salce, J. Thomasson, N. Bernhoeft, D. Braithwaite, and J. Flouquet, 2005, Phys. Rev. B 72, 024424.

Norman, M. R., Q. Si, Ya. B. Bazaliy, and R. Ramazashvili, 2003, Phys. Rev. Lett. 90, 116601.

Norman, M. R., 2005, Phys. Rev. B 71, 220405.

Nozières, P., 1974, J. Low Temp. Phys. 17, 31.

Nozières, P., and A. Blandin, 1980, J. Physique 41, 193.

Nozières, P., 1985, Ann. Phys. Fr. 10, 19.

Nozières, P., 2005, J. Phys. Soc. Jpn. 74, 4.

Oganesyan, V., S. A. Kivelson, and E. Fradkin, 2001, Phys. Rev. B 64, 195109.

Okumura, H., K. Kakurai, Y. Yoshida, Y. Ōnuki, and Y. Endoh, 1998, J. Mag. Mag. Mater. 177-181, 405.

Ōnuki, Y., and T. Komatsubara, 1987, J. Mag. Mag. Mater. 63-64, 281.

Oshikawa, M., 2000, Phys. Rev. Lett. 84, 3370.

Paalanen, M. A., and R. N. Bhatt, 1991, Physica B 169, 223.

Paalanen, M. A., S. Sachdev, R. N. Bhatt, and A. E. Ruckenstein, 1986, Phys. Rev. Lett. 57, 2061.

Pagliuso, P. G., R. Movshovich, A. D. Bianchi, M. Nicklas, N. O. Moreno, J. D. Thompson, M. F. Hundley, J. L. Sarrao and Z. Fisk, 2002, Physica B 312-313, 129.

Paglione, J., M. A. Tanatar, D. G. Hawthorn, E. Boaknin, R. W. Hill, F. Ronning, M. Sutherland, L. Taillefer, C. Petrovic, and P. C. Canfield, 2003, Phys. Rev. Lett. 91, 246405.

Park, T., F. Ronning, H. Q. Yuan, M. B. Salamon, R. Movshovich, J. L. Sarrao, and J. D. Thompson, 2006, Nature 440, 65 .

Paschen, S., T. Lühmann, S. Wirth, P. Gegenwart, O. Trovarelli, C. Geibel, F. Steglich, P. Coleman, and Q. Si, 2004, Nature 432, 881.

Paschke, C., C. Speck, G. Portisch, and H. v. Löhneysen, 1994, J. Low Temp. Phys. 97, 229.

Pastor, A. A, and V. Dobrosavljevic, 1999, Phys. Rev. Lett. 83, 4642.

Paul, I., C. Pépin, and M. R. Norman, 2007, Phys. Rev. Lett. 98, 026402.

Pedrazzini, P., D. Jaccard, G. Lapertot, J. Flouquet, Y. Inada, H. Kohara, and Y. Ōnuki, 2006, Physica B 378-380, 165.

Pépin, C., 2005, Phys. Rev. Lett. 94, 066402.

Petrovic, C., R. Movshovich, M. Jaime, P. G. Pagliuso, M. F. Hundley, J. L. Sarrao, Z. Fisk, and J. D. Thompson, 2001, Europhys. Lett. 53, 354.

Petrovic, C., P. G. Pagliuso, M. F. Hundley, R. Movshovich, J. L. Sarrao, J. D. Thompson, Z. Fisk, and P. Monthoux, 2001, J. Phys. Cond. Matter 13, L337.

Pfleiderer, C., G. J. McMullan, S. R. Julian, and G. G. Lonzarich, 1997, Phys. Rev. B 55, 8330.

Pfleiderer, C., M. Uhlarz, S. M. Hayden, R. Vollmer, H. v. Löhneysen, N. R. Bernhoeft, and G. G. Lonzarich, 2001, Nature 412, 58.

Pfleiderer, C., S. R. Julian, and G. G. Lonzarich, 2001, Nature 414, 427.

Pfleiderer, C., and A. D. Huxley, 2002, Phys. Rev. Lett. 89, 147005.

Pfleiderer C., D. Reznik, L. Pintschovius, H. v. Löhneysen, M. Garst, and A. Rosch, 2004, Nature 427, 227.

Pich, C., A. P. Young, H. Rieger, and N. Kawashima, 1998, Phys. Rev. Lett. 81, 5916.

Pietrus T., B. Bogenberger, S. Mock, M. Sieck, and H. v. 
Löhneysen, 1995, Physica B 206-207, 317.

Pollack, L., M. J. R. Hoch, C. Jin, E. N. Smith, J. M. Parpia, D. L. Hawthorne, D. A. Geller, D. M. Lee, R. C. Richardson, D. G. Hinks, and E. Bucher, 1995, Phys. Rev. B 52, R 15707.

Pomeranchuk, I. J., 1958, Sov. Phys. JETP 8, 361.

Privman, V., 1990, in "Finite Size Scaling and Numerical Simulation of Statistical Systems", ed. V. Privman (World Scientific, Singapore), p. 1.

Pruschke, T., R. Bulla, and M. Jarrell, 2000, Phys. Rev. B 61, 12799.

Quezel, S., J. Rossat-Mignod, B. Chevalier, P. Lejay, and J. Etourneau, 1984, Solid State Commun. 49, 685.

Quezel, S., P. Burlet, J. L. Jacoud, L. P. Regnault, J. RossatMignod, C. Vettier, P. Lejay, and J. Flouquet, 1988, J. Mag. Mag. Mater. 76-77, 403.

Raymond, S., L. P. Regnault, S. Kambe, J. M. Mignot, P. Lejay, and J. Flouquet, 1997, J. Low Temp. Phys. 109, 205.

Raymond, S., L. P. Regnault, J. Flouquet, A. Wildes, and P. Lejay, 2001, J. Phys. Cond. Matter 13, 8303.

Regnault, L. P., W. A. C. Erkelens, J. Rossat-Mignod, P. Lejay, and J. Flouquet, 1988, Phys. Rev. B 38, 4481.

Regnault, L. P., J. L. Jacoud, J. M. Mignot, J. RossatMignod, C. Vettier, P. Lejay, and J. Flouquet, 1990, J. Mag. Mag. Mater. 90-91, 398.

Rößler, U. K., A. N. Bogdanov, and C. Pfleiderer, 2006, Nature 442, 797.

Ronning, F., C. Capan, E. D. Bauer, J. D. Thompson, J. L. Sarrao, and R. Movshovich, 2006, Phys. Rev. B 73, 064519.

Rosch, A., A. Schröder, O. Stockert, and H. v. Löhneysen, 1997, Phys. Rev. Lett. 79, 159.

Rosch, A., 1999, Phys. Rev. Lett. 82, 4280.

Rosch, A., 2000, Phys. Rev. B 62, 4945.

Rosch, A., 2001, Phys. Rev. B 64, 174407.

Rosch, A., and P. C. Howell, 2005, Phys. Rev. B 72, 104510.

Rossat-Mignod, J., L. P. Regnault, J. L. Jacoud, C. Vettier, P. Lejay, J. Flouquet, E. Walker, D. Jaccard, and A. Amato, 1988, J. Mag. Mag. Mater. 76-77, 376.

Roussev, R., and A. J. Millis, 2001, Phys. Rev. B 63, 14050.

Sachdev, S., N. Read, and R. Oppermann, 1995, Phys. Rev. B 52, 10286.

Sachdev S., A. V. Chubukov, and A. Sokol, 1995, Phys. Rev. B 51, 14874.

Sachdev, S., and N. Read, 1996, J. Phys. Cond. Matter 8, 9723.

Sachdev, S., 1998, Phil. Trans. R. Soc. Lond. A, 356, 173.

Sachdev S., 1999, Quantum Phase Transitions, Cambridge University Press, Cambridge.

Sachdev, S., and E. R. Dunkel, 2006, Phys. Rev. B 73, 085116.

Sakakibara, T., H. Amitsuka, D. Sugimoto, H. Mitamura, and K. Matsuhira, 1993, Physica B 186-187, 317.

Sakai, O., Y. Shimizu, and T. Kasuya, 1989, J. Phys. Soc. Jpn. 58, 3666.

Sandeman, K. G., G. G. Lonzarich, and A. J. Schofield, 2003, Phys. Rev. Lett. 90, 167005.

Sandvik, A. W., 2002, Phys. Rev. Lett. 89, 177201.

Sarachik, M. P., in: Metal-insulator Transitions Revisited, ed. by P. P. Edwards and C. N. R. Rao (Taylor \& Francis, London, 1995) p. 79.

Saxena, S. S., P. Agarwal, K. Ahilan, F. M. Grosche, R. K. W. Haselwimmer, M. J. Steiner, E. Pugh, I. R. Walker, S. R. Julian, P. Monthoux, G. G. Lonzarich, A. Huxley, I. Shelkin, D. Braithwaite, and J. Flouquet, 2000, Nature
406, 587.

Scheidt, E.-W., T. Schreiner, K. Heuser, and G. R. Stewart, 1999, Physica B 259-261, 388.

Schlager, H. G., A. Schröder, M. Welsch, and H. v. Löhneysen, 1993, J. Low Temp. Phys. 90, 181.

Schlottmann, P., and P. D. Sacramento, 1993, Adv. Phys. 42, 641.

Schmalian, J., and M. Turlakov, 2004, Phys. Rev. Lett. 93, 036405.

Schrieffer, J. R., 1995, J. Low Temp. Phys. 99, 397.

Schrieffer, J. R., and P. A. Wolff, 1966, Phys. Rev. 149, 491.

Schröder, A., J. W. Lynn, R. W. Erwin, M. Loewenhaupt, and H. v. Löhneysen, 1994, Physica B 199-200, 47.

Schröder, A., G. Aeppli, E. Bucher, R. Ramazashvili, and P. Coleman, 1998, Phys. Rev. Lett. 80, 5623.

Schröder, A., G. Aeppli, R. Coldea, M. Adams, O. Stockert, H. v. Löhneysen, E. Bucher, R. Ramazashvili, and P. Coleman, 2000, Nature 407, 351.

Schuberth, E. A., J. Schupp, R. Freese, and K. Andres, 1995, Phys. Rev. B 51, 12892.

Seaman, C. L., M. B. Maple, B. W. Lee, S. Ghamaty, M. S. Torikachvili, J.-S. Kang, L. Z. Liu, J. W. Allen, and D. L. Cox, 1991, Phys. Rev. Lett. 67, 2882.

Sengupta, A. M., and A. Georges, 1995, Phys. Rev. B 52, 10295.

Sengupta, A. M., 2000, Phys. Rev. B 61, 4041.

Senthil, T., and S. Sachdev, 1996, Phys. Rev. Lett. 77, 5292.

Senthil, T., S. Sachdev, and M. Vojta, 2003, Phys. Rev. Lett. 90, 216403.

Senthil, T., M. Vojta, and S. Sachdev, 2004, Phys. Rev. B 69, 035111.

Senthil, T., S. Sachdev, and M. Vojta, 2005, Physica B 359$361,9$.

Senthil, T., A. Vishwanath, L. Balents, S. Sachdev, and M. P. A. Fisher, 2004, Science 303, 1490.

Senthil, T., L. Balents, S. Sachdev, A. Vishwanath, and M. P. A. Fisher, 2004, Phys. Rev. B 70, 144407.

Sichelschmidt, J., V. A. Ivanshin, J. Ferstl, C. Geibel, and F. Steglich, 2003, Phys. Rev. Lett. 91, 156401.

Shankar, R., 1994, Rev. Mod. Phys. 66, 129.

Si, Q., J. L. Smith, and K. Ingersent, 1999, Int. J. Mod. Phys. B 13, 2331.

Si, Q., S. Rabello, K. Ingersent, and J. L. Smith, 2001, Nature 413, 804.

Si, Q., S. Rabello, K. Ingersent, and J. L. Smith, 2003, Phys. Rev. B 68, 115103.

Sieck, M., C. Speck, M. Waffenschmidt, S. Mock, and H. v. Löhneysen, 1996, Physica B 223-224, 325.

Sieck, M., F. Huster, and H. v. Löhneysen, 1997, Physica B 230-232, 583.

Smith, J. L., and Q. Si, 1999, Europhys. Lett. 45, 228.

Smith, J. L., and Q. Si, 2000, Phys. Rev. B 61, 5184.

Sknepnek, R., T. Vojta, and M. Vojta, 2004, Phys. Rev. Lett. 93, 097201.

Sokolov, D. A., M. C. Aronson, W. Gannon, and Z. Fisk, 2006, Phys. Rev. Lett. 96, 116404.

Sondhi, S. L., S. M. Girvin, J. P. Carini, and D. Shahar, 1997, Rev. Mod. Phys. 69, 315.

Steglich, F., J. Aarts, C. D. Bredl, W. Lieke, D. Meschede, W. Franz, and H. Schäfer, 1979, Phys. Rev. Lett. 43, 1892.

Steglich, F., B. Buschinger, P. Gegenwart, M. Lohmann, R. Helfrich, C. Langhammer, P. Hellmann, L. Donnevert, S. Thomas, A. Link, C. Geibel, M. Lang, G. Sparn, and W. Assmus, 1996, J. Phys. Cond. Matter 8, 9909. 
Steglich, F., P. Gegenwart, R. Helfrich, C. Langhammer, P. Hellmann, L. Donnevert, C. Geibel, M. Lang, G. Sparn, W. Assmus, G. R. Stewart, and A. Ochiai, 1997, Z. Phys. B 103, 235.

Steglich, F., 2005, Physica B 359-361, 326.

Stewart, G. R., 2001, Rev. Mod. Phys. 73, 797.

Stewart, G. R., 2006, Rev. Mod. Phys. 78, 743.

Stewart, J. R., B. D. Rainford, R. S. Eccleston, and R. Cywinski, 2002, Phys. Rev. Lett. 89, 186403.

Stockert, O., H. v. Löhneysen, A. Rosch, N. Pyka and M. Loewenhaupt, 1998, Phys. Rev. Lett. 80, 5627.

Stockert, O., E. Faulhaber, G. Zwicknagl, N. Stüßer, H. S. Jeevan, M. Deppe, R. Borth, R. Küchler, M. Loewenhaupt, C. Geibel, and F. Steglich, 2004, Phys. Rev. Lett. 92, 136401.

Stockert, O., D. Andreica, A. Amato, H.S. Jeevan, C. Geibel, and F. Steglich, 2006, Physica B 374-375, 167.

Stockert, O., M. Enderle, and H. v. Löhneysen, 2006, unpublished.

Süllow, S., M. C. Aronson, B. D. Rainford, and P. Haen, 1999, Phys. Rev. Lett. 82, 2963.

Tabata, Y., T. Taniguchi, Y. Miyako, O. Tegus, A. A. Menovsky, and J. A. Mydosh, 2004, Phys. Rev. B 70, 144415.

Tahvildar-Zadeh, A. N., M. Jarrell, and J. K. Freericks, 1997, Phys. Rev. B 55, R3332.

Taillefer, L., G. G. Lonzarich, and P. Strange, 1986, J. Mag. Mag. Mater 54-57, 957.

Taniguchi, T., Y. Tabata, H. Tanabe, and Y. Miyako, 1998, J. Mag. Mag. Mater. 177-181, 419.

Tautz, F. S., S. R. Julian, G. J. McMullan, and G. G. Lonzarich, 1995, Physica B 206-207, 29.

Thompson, J. D., Z. Fisk, and G. G. Lonzarich, 1989, Physica B 161, 317.

Thompson, J. D., R. D. Parks, and H. A. Borges, 1986, J. Mag. Mag. Mater. 54-47, 377.

Trovarelli, O., C. Geibel, S. Mederle, C. Langhammer, F. M. Grosche, P. Gegenwart, M. Lang, G. Sparn, and F. Steglich, 2000, Phys. Rev. Lett. 85, 626.

Tsujii H., E. Tanaka, Y. Ode, T. Katoh, T. Mamiya, S. Araki, R. Settai, and Y. Ōnuki, 2000, Phys. Rev. Lett. 84, 5407.

Tsvelik, A. M., and P. B. Wiegmann, 1984, Z. Physik B 54, 201.

Ueda, K, 1977, J. Phys. Soc. Jpn. 43, 1497.

Uhlarz, M., C. Pfleiderer, and S. M. Hayden, 2004, Phys. Rev. Lett. 93, 256404.

Vargoz, E., P. Link, and D. Jaccard, 1997, Physica B 230232, 182 .

Vargoz, E., and D. Jaccard, 1998, J. Mag. Mag. Mater. 177181, 294.

Varma, C. M., and L. Zhu, 2006, Phys. Rev. Lett. 96, 036405.

v. Löhneysen, H., H. G. Schlager, and A. Schröder, 1993, Physica B 186-188, 590.

v. Löhneysen, H., T. Pietrus, G. Portisch, H. G. Schlager, A. Schröder, M. Sieck, and T. Trappmann, 1994, Phys. Rev. Lett. 72, 3262.

v. Löhneysen, H., M. Sieck, O. Stockert, and M. Waffenschmidt, 1996, Physica B 223-224, 471.

v. Löhneysen, H., 1996, J. Phys. Cond. Matt. 8, 9689.

v. Löhneysen, H., S. Mock, A. Neubert, T. Pietrus, A. Rosch, A. Schröder, O. Stockert, and U. Tutsch, 1998, J. Mag. Mag. Mater. 177-181, 12.

v. Löhneysen, H., A. Neubert, T. Pietrus, A. Schröder, O.
Stockert, U. Tutsch, M. Loewenhaupt, A. Rosch, and P. Wölfle, 1998, Eur. J. Phys. B 5, 447.

v. Löhneysen, H., C. Pfleiderer, T. Pietrus, O. Stockert, and B. Will, 2001, Phys. Rev. B 63, 134411.

v. Löhneysen, H., F. Obermair, C. Pfleiderer, and O. Stockert, 2002, Acta Physica Polonica B 34, 707.

Varma, C. M., P. B. Littlewood, S. Schmitt-Rink, E. Abrahams, and A. E. Ruckenstein, 1989, Phys. Rev. Lett. 63, 1996.

Varma, C. M., Z. Nussinov, and W. van Saarloos, 2002, Phys. Rep. 361, 267.

Vojta, T., D. Belitz, R. Narayanan, T. R. Kirkpatrick, 1997, Z. Phys. B 103, 451.

Vojta, M., C. Buragohain, and S. Sachdev, 2000, Phys. Rev. B 61, 15152.

Vojta, T., and R. Sknepnek, 2001, Phys. Rev. B 64, 052404.

Vojta, M., 2003, Rep. Prog. Phys. 66, 2069.

Vojta, T., 2003, Phys. Rev. Lett. 90, 107202.

Vojta, T., and J. Schmalian, 2005, Phys. Rev. B 72, 045438.

Vojta, M., 2006, Phil. Mag. 86, 1807.

Vojta, T., 2006, J. Phys. A 39, R143.

Vollmer, R., T. Pietrus, H. v. Löhneysen, R. Chau, and M. B. Maple, 2000, Phys. Rev. B 61, 1218.

Walstedt, R. E., H. Kojima, N. Butch, and N. Bernhoeft, 2003, Phys. Rev. Lett. 90, 067601.

Wiegmann, P. B., 1980, Sov. Phys. JETP Lett. 31, 392.

Wilhelm, H., K. Alami-Yadri, B. Revaz, and D. Jaccard, 1999, Phys. Rev. B. 59, 3651.

Wilson, K. G., 1975, Rev. Mod. Phys. 47, 773.

Wilson, S. D., P. Dai, D. T. Adroja, S.-H. Lee, J.-H. Chung, J. W. Lynn, N. P. Butch, and M. B. Maple, 2005, Phys. Rev. Lett. 94, 056402.

Wölfle, P., and A. Rosch, 2007, J. Low. Temp. Phys. 147, 165.

Yamamoto, S. J., and Q. Si, 2007, Phys. Rev. Lett. 99, 016401.

Yamase, H., and H. Kohno, 2000, J. Phys. Soc. Jpn. 69, 332 and 2151.

Yamase, H., V. Oganesyan, and W. Metzner, 2005, Phys. Rev. B 72, 035114.

Yeh, A., Y.-A. Soh, J. Brooke, G. Aeppli, T. F. Rosenbaum, and S. M. Hayden, 2002, Nature 419, 459.

Yelland, E. A., S. M. Hayden, S. J. C. Yates, C. Pfleiderer, M. Uhlarz, R. Vollmer, H. v. Löhneysen, N. R. Bernhoeft, R. P. Smith, S. S. Saxena, and N. Kimura, 2005, Phys. Rev. B 72, 214523 .

Young, B.-L., D. E. MacLaughlin, M. S. Rose, K. Ishida, O. O. Bernal, H. G. Lukefahr, K. Heuser, G. R. Stewart, N. P. Butch, P.-C. Ho, and M. B. Maple, 2004, Phys. Rev. B 70, 024401.

Yu, W., F. Zamborszky, J. D. Thompson, J. L. Sarrao, M. E. Torelli, Z. Fisk, and S. E. Brown, 2004, Phys. Rev. Lett. 92, 086403.

Yuan, H. Q., F. M. Grosche, M. Deppe, C. Geibel, G. Sparn, and F. Steglich, 2003, Science 302, 2104.

Yuan, H. Q., F. M. Grosche, M. Deppe, G. Sparn, C. Geibel, and F. Steglich, 2006, Phys. Rev. Lett. 96, 047008.

Zhu, J.-X., D. R. Grempel, and Q. Si, 2003, Phys. Rev. Lett. 91, 156404.

Zhu, L., M. Garst, A. Rosch, and Q. Si, 2003, Phys. Rev. Lett. 91, 066404. 